Zur Herkunft von ostseefinnisch $\tilde{o}$

Dissertation

zur Erlangung des philosophischen Doktorgrades

an der Philosophischen Fakultät der Georg-August-Universität Göttingen

vorgelegt von

Katja Mattsson

aus Hannover

Göttingen 2021 



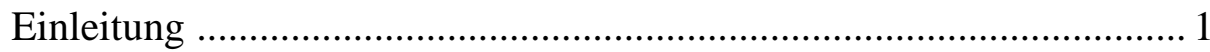

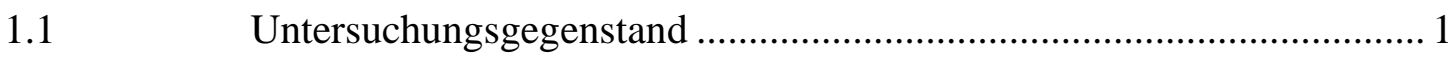

$1.2 \quad$ Aufbau

Untersuchtes Material und Auswahlkriterien ..................................... 2

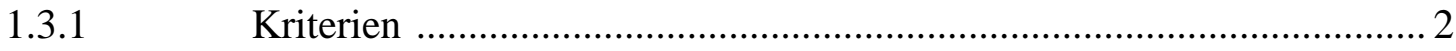

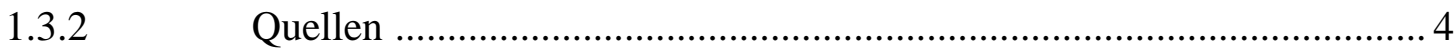

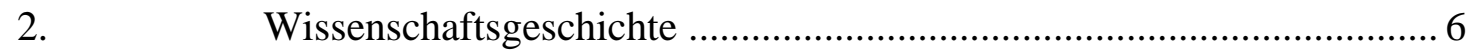

2.1 Das ostseefinnische $\tilde{o}$ als Laut der Grundsprache ................................ 6

2.2 Das ostseefinnische $\tilde{o}$ als sekundäre Entwicklung ............................... 14

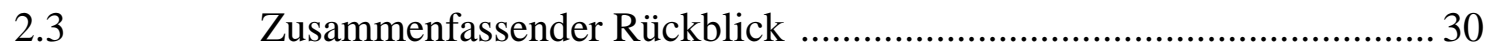

3. Zum Vokalinventar der ostseefinnischen Sprachen ….......................... 33

3.1 Der Vokalismus der ostseefinnischen Grundsprache ............................ 33

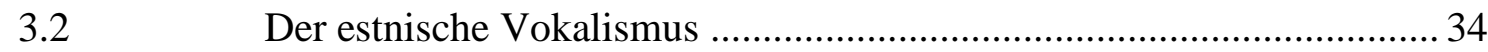

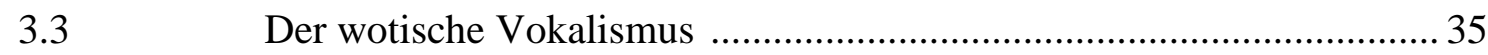

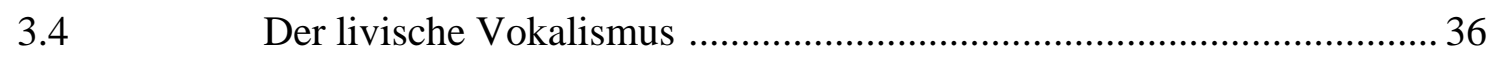

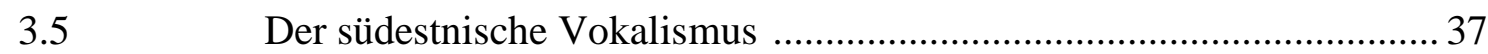

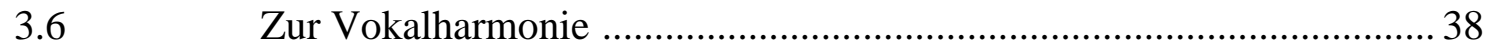

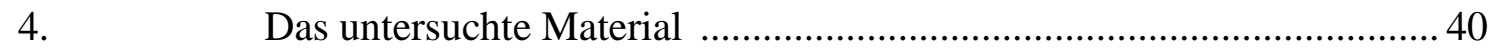

Zum Aufbau des Korpus ............................................................... 40

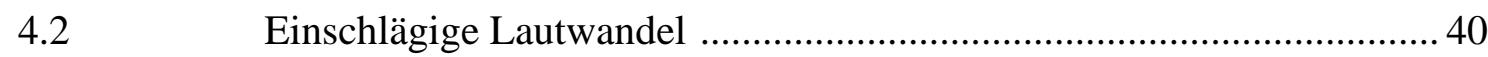

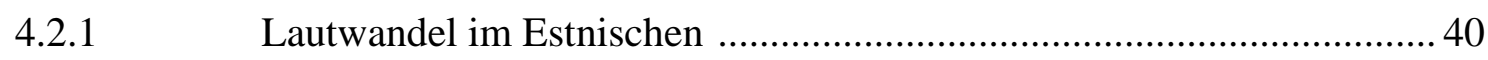

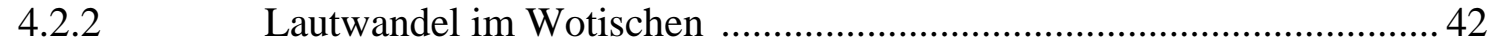

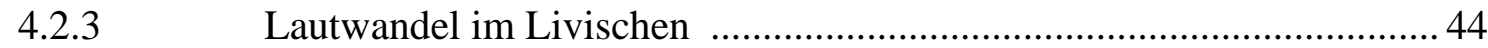

4.2.4 Lautwandel im Südestnischen ........................................................ 48

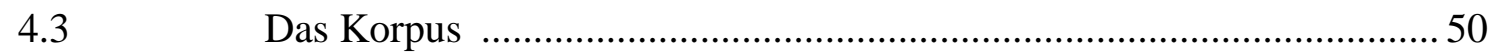

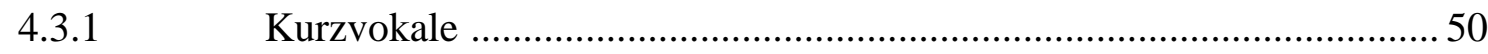

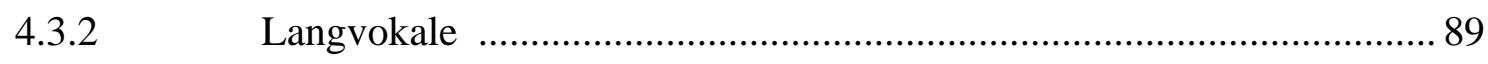

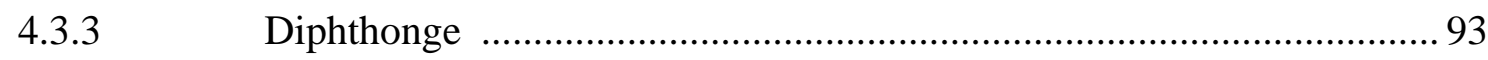

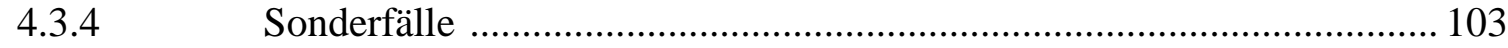


Kurzvokale 106

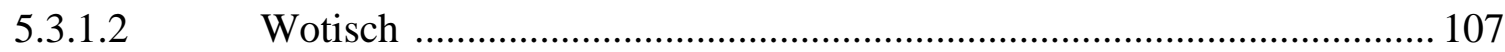

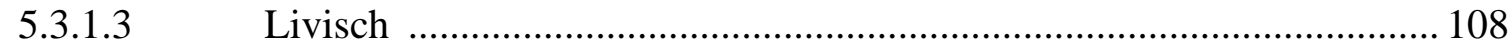

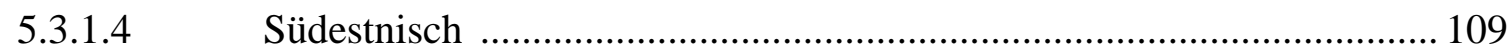

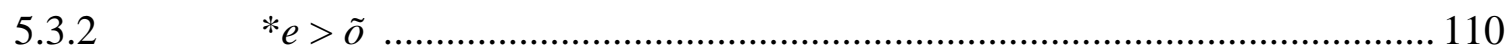

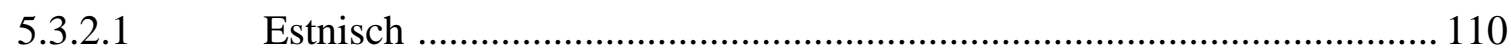

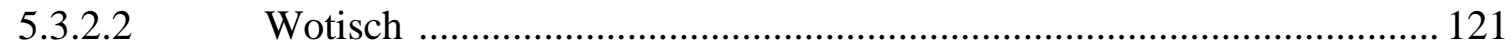

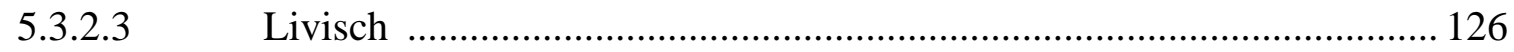

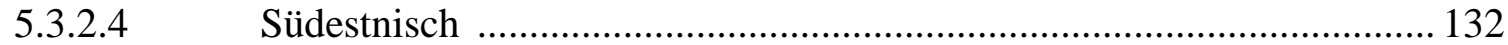

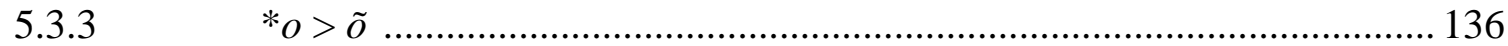

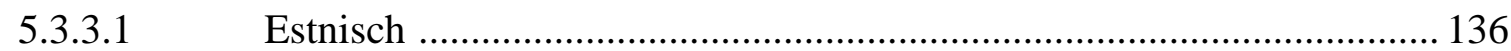

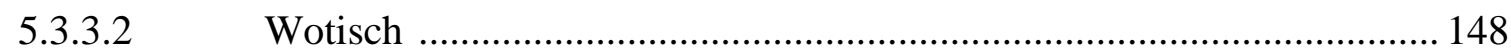

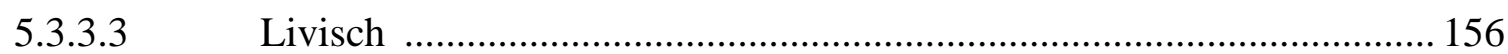

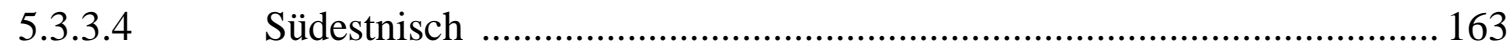

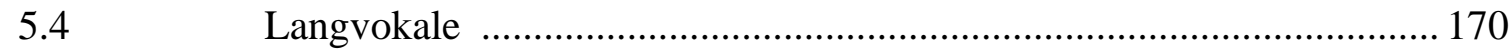

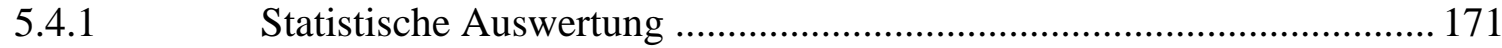

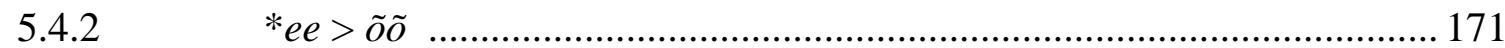

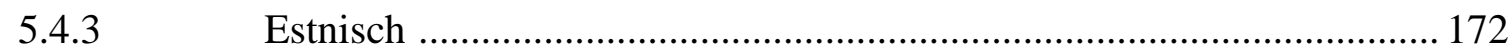

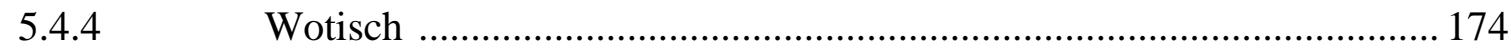

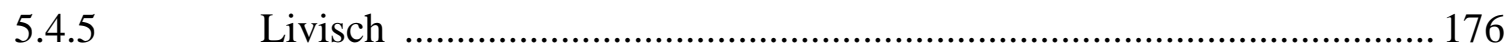

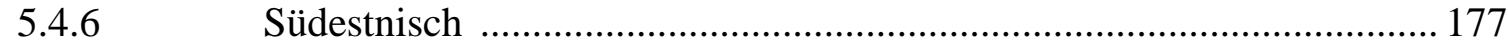

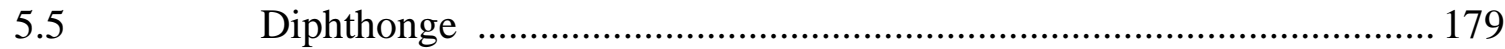

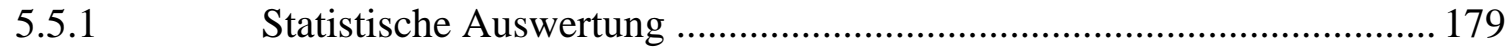

5.5.2 Diphthonge mit $u$ als zweitem Bestandteil ....................................... 179

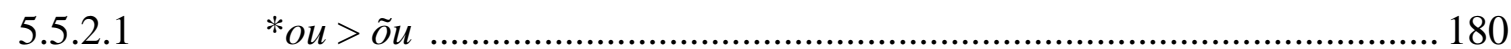

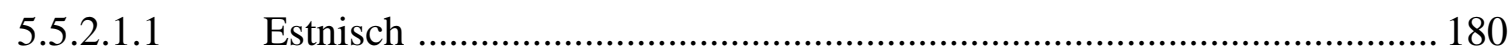

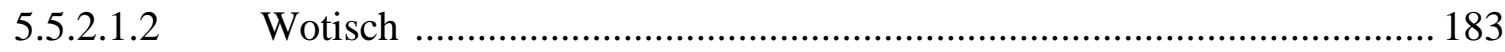

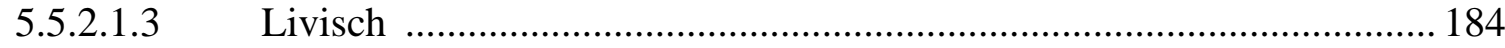




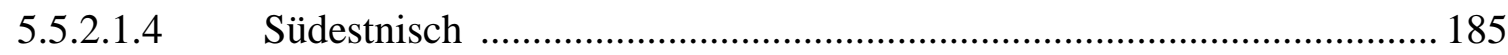

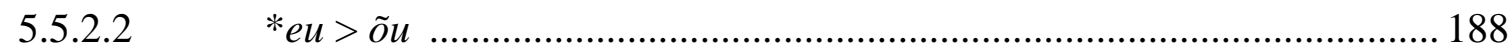

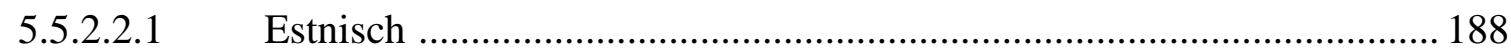

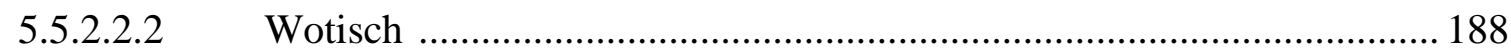

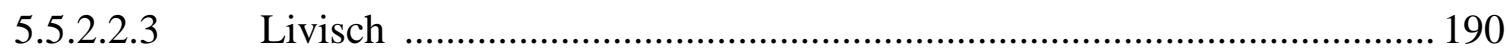

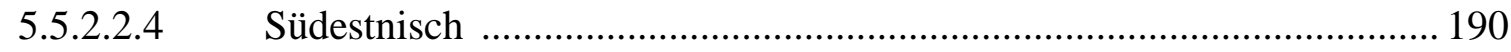

5.5.3 Diphthonge mit $i$ als zweitem Bestandteil ......................................... 191

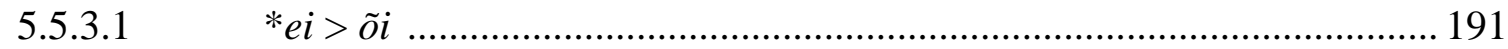

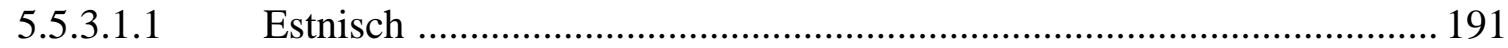

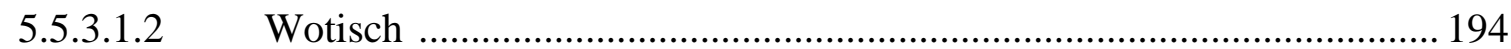

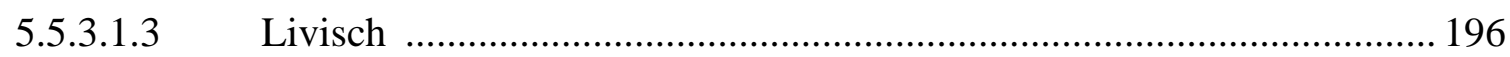

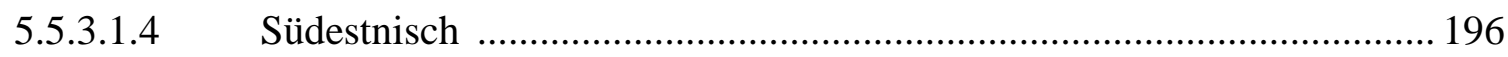

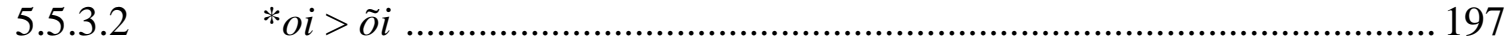

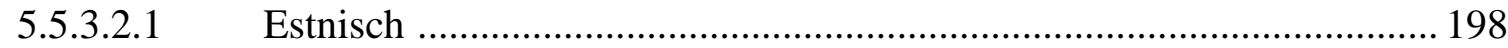

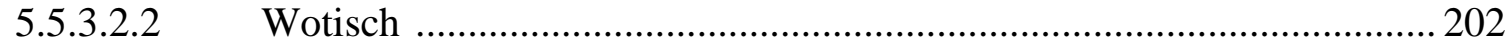

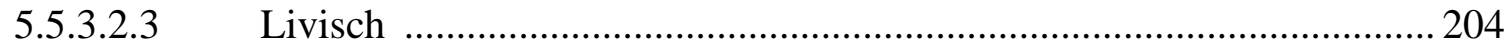

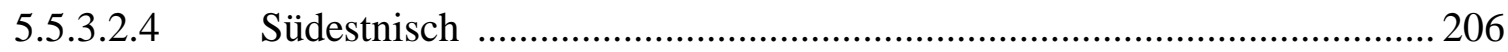

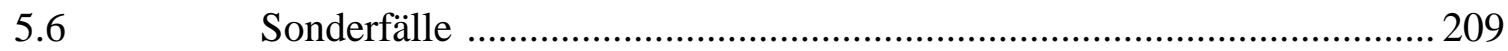

$5.7 \quad$ Entwicklungen mit marginaler Vertretung ….................................... 210

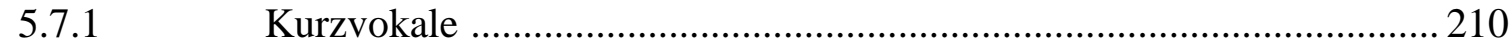

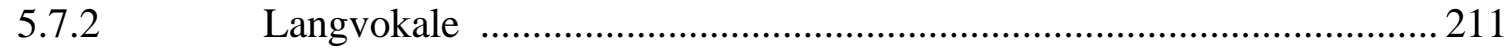

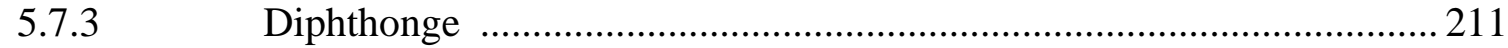

5.8 Zusammenfassung der Analyse ........................................................ 212

$5.9 \quad$ Belege mit weiterem Diskussionsbedarf ......................................... 215

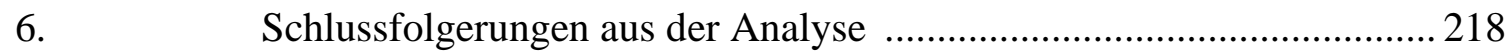

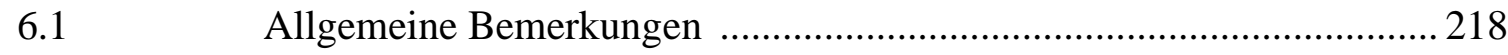

6.2 Zum Ablauf der unterschiedlichen Lautwandel …............................... 223

6.2.1 Vor der Entstehung von $\tilde{o}$ ansetzbare Entwicklungen ...........................223

6.2.2 Nach der Entstehung von $\tilde{o}$ ansetzbare Entwicklungen ........................225

6.2.3 Entwicklungen, die nicht datiert werden können ................................. 227

6.2.4 Zusammenfassender Überblick über die Lautwandel ........................... 231

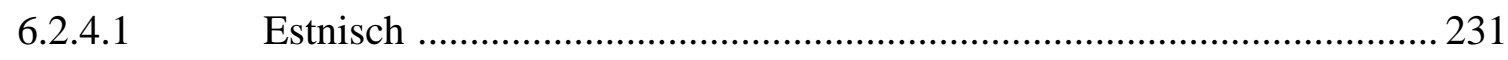

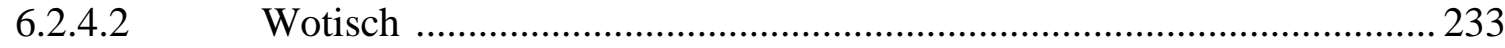




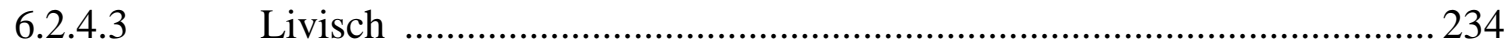

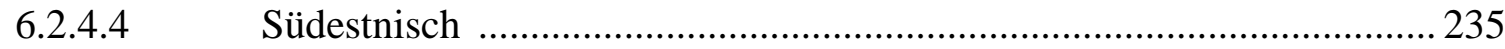

6.3 Zur Ausbreitung und zum Alter der Lautwandel zu $\tilde{o}$......................... 236

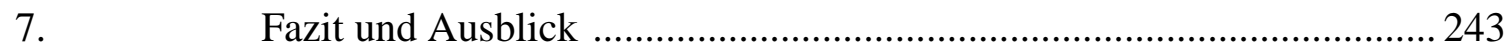

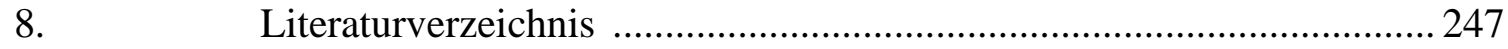

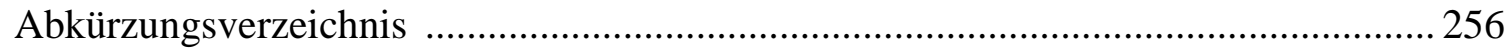

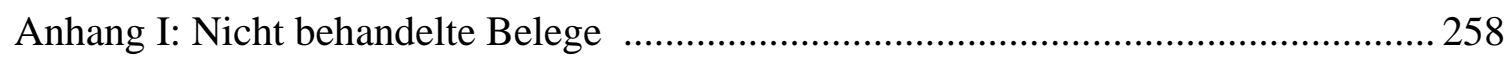

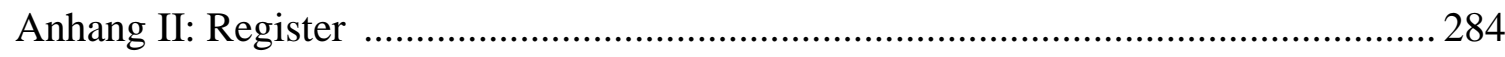




\section{$1.1 \quad$ Untersuchungsgegenstand}

Betrachtet man die ostseefinnischen Sprachen, so teilen sie sich aufgrund ihres Vokalinventars in zwei Gruppen auf: Es gibt die Südgruppe (Nord- und Südestnisch, Wotisch und Livisch), in der in der ersten Silbe ein Hinterzungenvokal $\tilde{o}[\gamma]$ auftritt, und die Nordgruppe (Finnisch, Karelisch, Wepsisch ${ }^{1}$, Ingrisch), die diesen nicht kennt. Die aus diesem Verhältnis resultierende Frage nach dem Alter des Lautes ist in der Finnougristik keine neue Fragestellung. Obwohl die Anfänge der Diskussion bereits 1877 zu finden sind, ist sie noch immer hochaktuell: Darin, ob es sich bei $\tilde{o}$ um einen ursprünglichen Vokal handelt, der bereits in der ostseefinnischen Grundsprache vorhanden gewesen ist und somit in einigen Sprachen später geschwunden sein muss, oder ob sich dieser Vokal erst in einer späteren Sprachstufe entwickelt hat, herrscht bisher keine Einigkeit.

Die dieser Untersuchung vorausgehenden Diskussionsbeiträge beziehen sich vornehmlich auf das Estnische und dabei insbesondere im Vergleich zum Finnischen auf die Entsprechungen $e$ o. Eine umfassende Arbeit, die über die Länge eines Artikels hinausgeht und dabei mehr als nur zwei der ostseefinnischen Sprachen und ihren Lautstand miteinander vergleicht, fehlt in der Finnougristik bisher. Ebenso beziehen sich die Arbeiten meist auf die Kurzvokale, jedoch tritt der Laut auch als Langvokal oder Bestandteil unterschiedlicher Diphthonge auf. Diese Problemstellung ausführlich und in einem umfassenderen Kontext zu behandeln, der zum ersten Mal auch das Wotische, Livische und Südestnische berücksichtigt, ist das Ziel dieser Dissertation. Sie macht es sich zur Aufgabe, die Entstehung des Lautes $\tilde{o}$ anhand eines Korpus bestehend aus allen vier Sprachen, in denen er auftritt, zu untersuchen und dessen Herausbildung nachzuvollziehen. Welche Entwicklungen können der Auslöser der Lautwandel sein? Warum ist die Verbreitung von $\tilde{o}$ in den Sprachen unterschiedlich stark ausgeprägt? Sind die Entwicklungen regelmäßig oder gibt es Abweichungen? Diese Fragen sollen im Rahmen der vorliegenden Betrachtung behandelt werden.

Neben den rein sprachwissenschaftlichen Bestandteilen dieser Ausführung und einer Untersuchung eines umfangreichen Korpus, der u.a. aus estnischen, wotischen, livischen und südestnischen Belegen besteht, lautliche Entwicklungen berücksichtigt und durch ein etymologisches

\footnotetext{
${ }^{1}$ Auch in einigen wepsischen Dialekten gibt es zwar einen solchen Laut. Die Bedingungen sind jedoch andere und eine eigenständige Entwicklung ist für diese Sprache ansetzbar (LAANEST 1982: 218), sodass das Wepsische hier nicht weiter behandelt wird.
} 
Fundament gestützt wird, soll als weiterer Aspekt ausführlich auf die Forschungsgeschichte dieses Themas eingegangen werden. Sie wird das Alter der Fragestellung und den Umfang der Problematik verdeutlichen sowie die eigentliche Diskussion darum aufbereiten. In den bisherigen Untersuchungen wurde selten mit einem größeren Korpus gearbeitet und, falls doch, stehen die Belege für die Leserschaft oft nicht zur Verfügung. In der Darstellung und Aufbereitung des Sprachmaterials leistet die vorliegende Arbeit in gewisser Hinsicht Pionierarbeit auf dem Gebiet der Erforschung des ostseefinnischen $\tilde{o}$.

\subsection{Aufbau}

Die Arbeit gliedert sich in mehrere Teile. Der erste Teil (Kapitel 2) behandelt zunächst den wissenschaftsgeschichtlichen Hintergrund des Themas, vertieft die Gewichtigkeit der Problematik und veranschaulicht die unterschiedlichen Positionen und Diskussionen. Im zweiten Teil (Kapitel 3) soll auf das Vokalinventar der untersuchten Einzelsprachen eingegangen werden. Im dritten Teil (Kapitel 4) werden die Belege, die als Grundlage der Analyse dienen, präsentiert und aufbereitet. Dabei stehen vor allem die Zusammenstellung der einzelnen Wörter auf etymologischer Basis und die lautlichen Entwicklungen im Vordergrund, während eine statistische Auswertung und Analyse der zuvor präsentierten Belege im vierten Teil (Kapitel 5) erfolgt. Die Ergebnisse der Analyse werden im fünften Teil (Kapitel 6) interpretiert und es werden Schlüsse zur Entwicklung der Lautwandel zu õ gezogen. Eine abschließende Zusammenfassung und Bewertung der Betrachtung erfolgen im sechsten und somit letzten Teil (Kapitel 7).

\subsection{Untersuchtes Material und Auswahlkriterien}

\subsubsection{Kriterien}

Zur Eingrenzung des einzelsprachlichen Materials sind folgende Punkte bei der Zusammenstellung des Korpus zu beachten: ${ }^{2}$

1) Es wurden ausschließlich Wörter mitsamt ihren Entsprechungen beachtet, in denen $\tilde{o}$ in der ersten Silbe des jeweiligen Wortstammes vorliegt.

2) Wörter, die in keinerlei etymologischen Zusammenhang gebracht werden können oder aufgrund mangelnder Kommentierung in den Wörterbüchern keiner Entsprechung in

\footnotetext{
${ }^{2}$ Belege, die nicht in das Korpus aufgenommen wurden, finden sich in Anhang 1.
} 
den anderen Sprachen zuzuordnen sind, werden nicht beachtet, z.B. est. óngima 'gierig fressen, schlingen' (EEW XII: 4028). Gleiches gilt ferner für derivierte Wörter, für die gesicherte Wortstämme der anzunehmend ursprünglichen Form bzw. ursprünglichen Wurzel vorliegen. Eine Ausnahme von dieser Ausschlussregel bilden allerdings Wörter, die in den heutigen Quellen ausschließlich in abgeleiteter Variante existieren und somit die einzig vorhandene Form des jeweiligen Wortstammes darstellen.

3) Auch aus der Betrachtung ausgeschlossen werden Lehnwörter aus dem Slawischen (genauer aus dem Russischen), die in der Position von ostseefinnisch $\tilde{o}$ in der Gebersprache ein $b l$ besitzen. Hier ist davon auszugehen, dass in den übernommenen Wörtern ein deutlicher Einfluss des russischen Lautstandes auf den ostseefinnischen Vokalismus wirkt und das Bild der tatsächlichen Lautentwicklung in den jeweiligen Einzelsprachen somit verfälscht werden kann. Das russische $b l$ ist dem ostseefinnischen $\tilde{o}$ lautlich gesehen recht nahe, sodass bei Lehnwörtern von einer mehr oder weniger direkten Adaption des Vokalismus der ersten Silbe der Gebersprache (mit einer entsprechenden Anpassung an den Vokalismus der Nehmersprache in Form von $\tilde{o}$ ) ausgegangen werden muss (vgl. auch BLOKLAND 2009: 330f.), z.B. est. rõõduma 'aushusten, rasselnd atmen' < russ. pыдámb 'schluchzen' (ebd. 243).

4) Verzichtet wird auch auf die Aufnahme niederdeutscher Lehnwörter, da diese nachweislich spät in das Estnische eintraten und somit keine Auskunft über einen alten Lautstand geben können (vgl. VIITSO 1998b: 146).

Behandelt wird in diesem Rahmen ausschließlich das Auftreten von $\tilde{o}$ in der ersten Silbe. Es ist davon auszugehen, dass es sich bei der Entstehung des Lautes in nichtersten Silben um eine sekundäre Entwicklung handelt: Im Estnischen tritt $\tilde{o}$ in nichterster Position nicht auf (VIITSO 2003: 21). Lediglich einige jüngere Lehnwörter bilden die Ausnahme, wenngleich man es hierbei eindeutig mit einem Substitut der fremden Lautung der Gebersprache zu tun hat, vgl. z.B. est. šašlõkk 'Schaschlik' (< russ. шашлык 'Schaschlik, Kebab' < tat. šyšlyk 'Bratspieß' (BLOKLAND 2009: 271)). Im Wotischen ist der Laut $\tilde{o}$ in der zweiten Silbe sekundär durch die Vokalharmonie bedingt (vgl. LAUERMA 1993: 54f.). Belege, in denen die erste Silbe vordervokalisch ist und in nichtersten Silben $\tilde{o}$ vorliegt, liegen nicht vor. Eine sog. $\tilde{o}$-Harmonie tritt auch im Südestnischen auf, sofern die Vokale der ersten Silbe Hintervokale sind (KEEM 1997: 7). Das livische $\tilde{o}$ der nichtersten Silben stellt ein orthographisches Problem dar. Während man in LELS das Graphem $\tilde{o}$ verwendet, zeigt sich bei einem Blick in KETTUNENS Wörterbuch (LW), dass 
es mitnichten derselbe Vokal ist wie jener der ersten Silbe. Vielmehr liegt ein reduzierter illabialer Hintervokal vor, der dort als â notiert wird (ebd. XVIII).

\subsubsection{Quellen}

Das Korpus wurde auf Grundlage der nachfolgenden Quellen zusammengestellt: ${ }^{3}$

\section{ESTNISCH}

- Eesti etümoloogiasõnaraamat (EES; Estnisches Etymologiewörterbuch, 2012),

- Estnisches Etymologisches Wörterbuch (EEW I-XII, 1982-1983),

- Eesti murrete sõnaraamat (EMS; Estnisches Dialektwörterbuch, 1994-) ${ }^{4}$,

- Väike murde sõnastik (VMS; Kleines Dialektwörterbuch, 1989);

WOTISCH $^{5}$

- Vadja Keele Sõnaraamat (VKS; Wörterbuch der wotischen Sprache, 2013) ${ }^{6}$,

- LAURi KeTtunens Vatjan Kielen Mahun murteen sanasto (Wörterverzeichnis des Mahu-Dialekts der wotischen Sprache, 1986);

\section{LIVISCH}

- Līvõkiel-ēstikiel-lețkiel sõnārōntõz (LELS; Livisch-Estnisch-Lettisches Wörterbuch, 2012), ${ }^{7}$

- Livisches Wörterbuch mit grammatischer Einleitung (KETTUNEN, 1938) ${ }^{8}$,

- Salis-Livisches Wörterbuch (WINKLER und PAJUSALU, 2009), wenngleich das Salis-Livische selbst kein Betrachtungsgegenstand ist;

\footnotetext{
${ }^{3}$ Bei der Darstellung der Belege aus den Einzelsprachen wird die Schreibweise aus der jeweiligen Quelle übernommen. So findet sich beispielsweise bei einigen Belegen die Transkription nach KETTUNEN (LW) oder im Südestnischen ein . als Markierung der Überlänge sowie- $q$ als Markierung des Glottalverschlusses (EVS 11ff.).

${ }^{4}$ Es ist mittlerweile zu großen Teilen im Internet abrufbar: http://www.eki.ee/dict/ems/pdf.html (Zuletzt abgerufen am 09.11.2020).

${ }^{5}$ Das Inkeroismurteiden sanakirja (IMS; Wörterbuch der ingrischen Dialekte, 1971) von NIRVI wurde bei der Zusammenstellung und Überprüfung der abweichenden Formen im Wotischen ebenfalls zurate gezogen, brachte jedoch an betreffenden Stellen keine neuen Erkenntnisse und fand daher keinen Eingang in das Korpus.

${ }^{6}$ Das Wörterbuch verwendet eine neue, an das Estnische angelehnte Schreibung und ist die fertiggestellte Ausgabe des ursprünglich mehrbändigen wotischen Wörterbuches gleichen Namens, dessen erster Band bereits im Jahr 1990 erschien (VKS 20).

${ }^{7}$ Die Schreibung der livischen Korpuseinträge wurde bei der Zusammenstellung soweit möglich an die Orthografie dieser Ausgabe angelehnt, da diese nach den Regeln der heute gültigen livischen Rechtschreibung arbeitet (LELS 15). Die Orthografie wurde im Rahmen von zwei Konferenzen 2005 normiert (BLUMBERGA 2011: 148), obwohl bereits da die Anzahl der Muttersprachler schwindend gering war. Jedoch werden schon seit den 1980ern von verschiedenen Gesellschaften Livisch-Sprachkurse organisiert. Die Identifikation mit dem Livischen hängt BLUMBERGA (ebd. 147) zufolge nicht mit der Sprache zusammen. Im Bereich der Kultur gibt es dagegen eine Vielzahl an Aktivitäten und Gesellschaften (ebd. 148-153).

${ }^{8}$ Die Einträge, die von Relevanz sind, jedoch ausschließlich bei KETTUNEN vorliegen, werden nach seiner Transkription betrachtet.
} 
SÜDESTNISCH ${ }^{9}$

- Võro-eesti synaraamat (VES; Võru-Estnisches Wörterbuch, 2002),

- Eesti-võru sõnaraamat (EVS; Estnisch-Võru-Wörterbuch, 2014),

- $\quad$ EMS, VMS

\section{WEITERE ERGÄNZUNGEN}

Etymologische Belege

- Suomen Sanojen Alkuperä (SSA; Der Ursprung der finnischen Wörter, 1992, 1995, 2000),

- Uralisches Etymologisches Wörterbuch (UEW I und II, beide 1988),

- Eesti Etümoloogiasõnaraamat (s.o.);

Zu Lehnwörtern

- Lexikon der älteren germanischen Lehnwörter in den ostseefinnischen Sprachen (LÄGLOS I-III, 1991, 1996 und 2012),

- Vene Laensõnad Eesti Murretes, (Russische Lehnwörter in den estnischen Dialekten, 2000) von MARI MUST,

- The Russian Loanwords in Literary Estonian (2009) von ROGIER BLOKLAND;

DEUTSCHSPRACHIGE ÜBERSETZUNGEN DER BELEGE

- Etymologische Wörterbücher (SSA I-III und EEW I-XII)

- Livisches Wörterbuch (LW)

- Gebrauchswörterbücher von KOKLA [et al.] (1972), KANN [et al.] (1972 und 2003) sowie FORSSMAN (2005) zum Estnischen

- Suomalais-Saksalainen Suursanakirja (Finnisch-deutsches Großwörterbuch) von KATARA und SCHELlBACH-KoPRA (1974) sowie Suomi-saksa-suomi-sanakirja (Wörterbuch Finnisch-Deutsch-Finnisch) von BöGER [et al.] (2008) zum Finnischen

\footnotetext{
${ }^{9}$ Auch das Seto sõnastik (Setu-Wörterverzeichnis, 2016) von INGE KäSI wurde bei der Erstellung des Korpus zurate gezogen, jedoch nicht zitiert, da es an den fraglichen Stellen keine neuen Erkenntnisse liefert.
} 
Auf die Anfänge der Erforschung und der wissenschaftlichen Diskussion von $\tilde{o}$ vom Ende des 19. Jahrhunderts bis in die 1940er Jahre geht bereits ERKKI ITKONEN (1945) in seinem Artikel Onko kantasuomessa ollut keskivokaaleja? (Hat es im Urostseefinnischen Mittelvokale gegeben?) ausführlich ein. Es zeigt sich schon an dieser Stelle, dass das Thema die Wissenschaft seit Langem umtreibt und bisher keine Antwort auf die Frage nach dem Alter und der Herkunft von $\tilde{o}$ gefunden wurde. ALO RAUN (1971) greift ITKONENS Ausführungen später in seinen Essays in Finno-Ugric and Finnic Linguistics auf und führt sie fort. Die Forschungsgeschichte soll hier aus diesem Grund nicht in chronologischer Reihenfolge wiedergegeben werden, weil sich diese zu Genüge in den beiden genannten Arbeiten wiederfindet. Viel wesentlicher ist eine Gegenüberstellung der beiden erwähnten Positionen, um die Uneinigkeit zu diesem weiterhin aktuellen Thema innerhalb der sprachwissenschaftlichen Forschung deutlich zu machen. Einerseits gibt es diejenigen, die annehmen, dass es sich bei $\tilde{o}$ um einen Laut aus der ostseefinnischen Grundsprache handelt und in den Norddialekten abgebaut wurde. Im kompletten Gegensatz dazu stehen die Annahmen, dass $\tilde{o}$ eine sekundäre Entwicklung ist, die erst nach einer Aufteilung des Sprachzweigs aufkam.

Aufgenommen werden konnten in diesen Überblick nur Beiträge, die bis einschließlich Ende 2018 erschienen sind. Jüngere Publikationen konnten bei der Zusammenstellung nicht mehr berücksichtigt werden.

\subsection{Das ostseefinnische $\tilde{o}$ als Laut der Grundsprache}

Bereits bei den ersten Wissenschaftlern, die sich mit diesem Laut näher befassten, nämlich ARVID GENETZ und VILHELM THOMSEN (ITKONEN 1945: 159), lässt sich eine deutliche Positionierung in der Frage nach dem Alter von õ erkennen. GENETZ (1877: 13f.) stellt in seinem Werk Versuch einer karelischen Lautlehre zunächst einen kurzen Vergleich des Vokalismus einiger ostseefinnischer Sprachen auf, um zu belegen, dass es sich bei der Unterscheidung von $i$ und $i$, sowie $e$ und $e(\tilde{o})$ nicht nur um Varianten des gleichen Lautes handele. Insgesamt nennt er vier konkrete Beispiele, nämlich die Entsprechungen zu fi. vesa 'Spross', fi. tempaa 'reißen, rücken3SGPRÄs', fi. merta 'Reuse' und fi. hepo 'Pferd', und weist sie neben dem Finnischen noch im Karelischen, Wotischen, Estnischen und Livischen nach (ebd. 13). Von einer umfassenden Kor- 
pusanalyse kann hier jedoch noch nicht die Rede sein, da sein Belegmaterial mit nur vier Beispielen dafür unzureichend ist. GENETZ nimmt an, dass $\tilde{o}$ ein ursprünglicher Vokal gewesen sein muss und geht sogar so weit, ihn in der Frage nach dem Alter der Vokale als Ausgangspunkt für die anderen zu bezeichnen. Dabei sagt er selbst, dass „sich dafür allerdings keine historischen Belege aufstellen [lassen]“", da die sprachlichen Quellen noch zu neu seien (ebd. 13). Daher beschreibt GENETZ zunächst die Art und Weise, wie man wohl den Laut am besten produzieren kann:

Da bietet sich das ehstnische und wotische $\tilde{o}$ ganz natürlich als Ausgangspunkt für die anderen dar. Aus diesem, für die abendländischen Sprachen fremden, jedoch in mehreren uralaltaischen Sprachen, - unter anderen im Türkischen und Samojedischen — vorkommenden Vocal könnte $o$ also entstanden sein, dass man, um die Verengerung des Lautcanals in dem gutturalen Articulationspunkte zu erleichtern, den unteren Kiefer und zugleich die untere Lippe sich hat heben lassen, wodurch labiale Vocalstellung zugleich mit der gutturalen entstanden ist. (ebd. 13f.)

Die Artikulation des livischen $\tilde{o}$ wird von ihm sogar gesondert behandelt:

Das livische $o$ wiederum - im Fall unsere physiologische Analyse dieses Vocals richtig ist - wäre aus diesem $e$ durch eine gleichartige Accession der Labialvocalstellung wie bei $o$ gebildet. (ebd. 14)

Eine konkrete Begründung für seine Annahme, dass $\widetilde{o}$ alt sei, nennt er nicht und er versucht auch nicht, diese zu beweisen. Später revidiert GENETZ (1897: 50) seine Meinung zum Alter von $\tilde{o}$ und sagt, dass dieser estnische, wotische und livische Laut jünger als aus dem Urostseefinnischen sein müsse. Eine genauere Begründung für sein Umschwenken gibt GENETZ jedoch in seinen Ausführungen nicht und er geht auch nicht darauf ein, dass er früher andere Ansichten vertreten hat. Vielmehr stellt er seine Aussage einfach als gegeben dar.

VILHELM THOMSEN beschäftigt sich in seinem Werk Beröringer mellem de finske og de baltiske (litauisk-lettiske) sprog: en sproghistorisk undersøgelse (1890) näher mit dem Livischen. ${ }^{10}$ In einem Exkurs zum Vokalismus dieser Sprache nennt er dort zwei verschiedene Varianten von o: Er beschreibt zunächst den Laut $\tilde{o}$ als ein unreines oder auch „gemischtes“ $o$, gleich dem, den man im Estnischen finde. Dieser Laut, den es nur in der „Kolkenischen Mundart“ (also dem Kurland-Livischen) gebe, sei immer kurz und stehe in Verbindung mit einem vorausgehenden $v$ (THOMSEN 1931: 66f.), ergo einem labialen Laut.

\footnotetext{
${ }^{10}$ Zitiert wird hier die etwa 40 Jahre später erschienene Übersetzung dieser Arbeit: Berührungen zwischen den finnischen und den baltischen (litauisch-lettischen) Sprachen - Eine sprachgeschichtliche Untersuchung (THOMSEN 1931).
} 
In seinen Betrachtungen führt THOMSEN außerdem den Laut $\phi$, der mit dem $\varrho$ im Wörterbuch von SJÖGREN-WIEDEMANN identisch sei, an. Er wird als zwischen $\ddot{o}$ und $\ddot{u}$ liegend beschrieben, unterscheide sich dabei stark von $\ddot{o}$, noch stärker von $\tilde{o}$. In unbetonten Silben entspreche $\phi$ rein etymologisch betrachtet einer Vielzahl von Vokalen, in Stammsilben meist dem estnischen $\tilde{o}$. So nennt er z.B. liv. $v \phi r g$ [sic!] 'Netz' - fi. verkko - est. võrg oder liv. $n \phi v$ [sic!] 'Ratschlag' - fi. neuvo - est. nõu (ebd. 67f.). Weiterhin beschreibt THOMSEN (ebd. 162f.) auch ein ,gemischtes“ $e$, das es im Estnischen und Wotischen noch als $\tilde{o}$ gebe, im Livischen dagegen als $\phi(i \ddot{)})$. Es entspreche dem ursprünglich kurzen $e$ im Litauischen und Lettischen. THOMSEN verweist in der Frage nach dem Ursprung von $\tilde{o}$ auf GENETZ und meint, dass sich die Entsprechungen des lit.lett. $e$ im fi. $e$,oder vielmehr [einem] der beiden Laute, die jetzt beinahe oder ganz in f. $e$ zusammengefallen sind“ (ebd. 162) wiederfinden. Auch THOMSEN geht also von einem ursprünglichen $\tilde{o}$ aus, ohne seine Annahme zu explizieren oder sich auf einen umfassenden Datensatz zu stützen.

Als dritter Wissenschaftler ist in dieser Frage EEMIL NeSTOR SETÄLÄ zu nennen. Nicht zuletzt in seiner Mitteilung Über Quantitätswechsel im Finnisch-Ugrischen (SETÄLÄ 1896) macht er sich in einem Nachtrag zum vorfinnischen Vokalismus auch über die Frage nach dem Alter von $\tilde{o}$ Gedanken. Er nimmt diverse unterschiedliche Laute, darunter $\underline{i}$ und $e$, für die Grundsprache an und belegt letztere äußerst knapp durch Beispiele aus dem Finnischen, Mordwinischen und Samischen. Von $\underline{i}$ behauptet er (ebd. 36), es sei im Finnischen mit $i$, im Mordwinischen mit dem ursprünglichen $u$ und im Samischen mit jeweils beiden Lauten zusammengefallen. Im Hinblick auf das $e$ gebe es jedoch nicht viel Material. Für einen Teil der ostseefinnischen Sprachen, also Finnisch, Karelisch und Wepsisch, nimmt er ein Zusammenfallen von $e$ und $e$ an, während es im Estnischen, Livischen und Wotischen eine klare Abgrenzung dieser Laute gebe (ebd. 37f.). Dass die verschiedenen $e$-Laute zusammengefallen seien, behauptet er ebenso für das Samische, während es im Mordwinischen meist einen Hintervokal, mitunter aber auch $i$ als Vertretung gebe. Eine Erklärung oder Herleitung für seine Annahmen liefert auch SETÄLÄ nicht.

SETÄLÄ (1896: 24) versprach in seinem Artikel, im Rahmen seines mehrbändigen Werkes Yhteissuomalainen Ä̈̈nnehistoria (Gemeinostseefinnische Lautgeschichte. 1899) neben der ausführlichen Beschreibung des ostseefinnischen Konsonantismus in den ersten beiden Bänden auch einen eigenständigen Band zum Vokalismus herauszugeben. Dort wollte er die Ausführungen seines Artikels detaillierter erklären. Dieses Versprechen hielt er jedoch nicht. Der dritte 
Band erschien nie, da er zu jenem Zeitpunkt der Meinung war, seine Thesen zur Stufenwechseltheorie seien überholt (AUTIO 1998).

Auch LAURI KeTtUNEN ist der Meinung, dass es sich bei $\tilde{o}$ um einen primären Laut handeln müsse. Er sieht diesen Vokal sowohl in Vatjan kielen äännehistoria (Lautgeschichte der wotischen Sprache. 1915) ${ }^{11}$ als auch in Livisches Wörterbuch mit grammatischer Einleitung, das 1938 erschien, als aus dem Urostseefinnischen bewahrt an. Beschrieben wird $\tilde{o}$ im Livischen (LW XXIV) und im Wotischen (VKÄ 126) als eine urfinnische Erscheinung. Diese Aussage zeigt sich im Wörterbuch auch bei der Rekonstruktion der gemeinsamen ostseefinnischen Wortstämme der Beispielwörter. So führt KETTUNEN (LW 283) beispielsweise das Wort liv. põ'ddõrz (in KETTUNENS Transkription $p e^{\prime} d d \hat{r} r(z)$ ), est. põder 'Elentier' zurück auf ein rekonstruiertes *petra. Zwar schließt er nicht aus, dass es auch eine Entwicklung von $o>e(\tilde{o})$ gegeben habe, sagt aber, dass diese selten sei (ebd. XXIV). Eine Begründung seiner Annahme, dass o primär sei, bleibt er jedoch in beiden Werken schuldig.

In seiner Lautgeschichte des Estnischen (Eestin kielen äännehistoria ${ }^{12}$ ) geht KETTUNEN (EKÄ 126f.) ebenfalls schon von einem ursprünglichen Laut $\tilde{o}$, also einem hinteren $e$, aus. Als besonders interessant nennt er in seiner Monografie eine Reihe von Wörtern, die im Vokalismus der ersten Silbe $\tilde{o}$ beinhalten, in der Folgesilbe hintervokalisch sind und denen im Finnischen dort $e$ entspricht, z.B. est. võl $\mid g,-a$ 'Schuld', est. $v \tilde{o} r \mid k$, - $g u$ 'Netz' oder est. põrn, - $a$ 'Milz'. Zu diesen Beispielen fügt er an, dass ihr Erstsilbenvokal zwar als hinteres $e$ bezeichnet wird und somit anzunehmen sei, dass dieser aus dem ursprünglichen Vokalismus bewahrt worden ist, er jedoch tiefer ausgesprochen wird als das $\operatorname{sog}$. hintere $e$, wie der ursprüngliche Laut auch bezeichnet wird. Als Beweis dafür sieht er, dass statt $\tilde{o}$ in Dialekten $o$ oder $\ddot{o}$ gleichermaßen stehen können. Auch wenn KETTUNEN seine Analyse nicht weiter ausführt und seine Erkenntnisse eher eine Randbemerkung bleiben, so ist er der erste, der einen Zusammenhang zwischen dem Lautwandel der ersten Silbe und dem Hintervokal in der nachfolgenden Silbe herstellt.

Ferner erklärt KETTUNEN (ebd. 131) auch, dass es in Wörtern wie est. kõrge 'hoch' oder est. õlg 'Schulter' einen Wandel $o>\tilde{o}$ gegeben habe. Als Beweis stützt er sich dabei auf die dialektale Vertretung des Lautes und sagt, dass $o$ in den südestnischen Dialekten oftmals in Wörtern auftrete, in denen in anderen Dialekten $\tilde{o}$ stehe. Für das Livische weist er als erster an dieser Stelle

\footnotetext{
${ }^{11}$ Die erste Auflage von Vatjan kielen äännehistoria erschien 1915. Zitiert wird in dieser Arbeit die überarbeitete zweite Auflage aus dem Jahr 1930.

${ }^{12}$ Die erste Auflage von KeTtunENS estnischer Lautgeschichte erschien 1916. Hier wird nach der dritten Auflage von 1962 zitiert.
} 
darauf hin, dass es den Wandel $o>\tilde{o}$ von wenigen Ausnahmen abgesehen nicht kenne. Daraus schließt KeTtunen (ebd. 132), dass diese Entwicklung schon am Ende der ostseefinnischen Periode (alkusuomalainen aika) im Wotischen und Ostestnischen stattgefunden haben müsse, während $o$ im Westestnischen bewahrt worden sei. Anscheinend vermutet er in den unterschiedlichen Lautständen des Estnischen also verschiedene dialektale Entwicklungen und scheint von einer Art Kontinuum vom Wotischen über das Ostestnische zum Westestnischen bis hin zum Livischen auszugehen.

KETTUNEN ist der erste, der versucht, konkrete Lautwandel deutlich zu benennen. Auffallend ist dabei, dass er sich vor allem mit den Entsprechungen $e \sim \tilde{o}$ beschäftigt, denn diese sind regelmäßig und verhältnismäßig leicht zu begründen. Die auf den ersten Blick nur schwer erklärbaren Entsprechungen $o \sim \tilde{o}$ erkennt KETTUNEN zwar und benennt sie am Rande, dennoch lässt er sie weitestgehend außer Acht und behandelt sie nicht ausführlich genug, um von einer fundierten Analyse sprechen zu können.

LAURI POSTI (1942: 17) schreibt, „das späturfinnische $e$ ist im Westlivischen (und im Übergangsdialekt Ii.) erhalten“"13 und vertritt somit die gleiche Meinung wie KETTUNEN einige Jahre vor ihm. Als Beispiele nennt er liv. ęcrà, livW. irà 'der Geifer, welcher Böcken und Schweinen

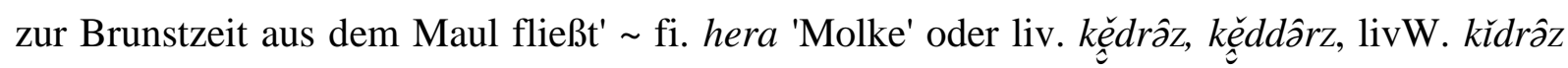
'Schlittenstütze zwischen Sohlen und Seitenhölzern' fi. ketara 'Strebe'. Auch PosTI lässt die Begründung für ein primäres $\tilde{o}$ aus und weist nur auf eine Entwicklung von livW. $e(\tilde{o})>\ddot{u}$ hin, die seiner Meinung nach spät vonstattengegangen sein muss, weil sie auch das sekundär herausgebildete $e$ mit eingeschlossen habe. Wichtig sei dabei, dass es sich nicht um die gleiche Entwicklung handle, wie es im Salis-Livischen der Fall ist, da sie im Kurlandlivischen vermutlich aufgrund von Fremdeinfluss vor sich gegangen sei (ebd. 17f.).

Wie bereits THOMSEN erkennt auch POSTI (ebd. 11), dass die Labialität der lautlichen Umgebung des Vokals der ersten Silbe in einigen Fällen eine Rolle spielen kann. Er denkt, dass es eine Entwicklung von $o>\tilde{o}$ gegeben haben müsse, wenn darauf ein $v$ folgte (und nicht wie THOMSEN nur nach einem vorausgehenden v). Als Beispiel nennt er u. a. liv. kęurâ 'krumm, gekrümmt' fi. kovera 'konkav, ausgehöhlt' est. kõver 'krumm'. Das Einbeziehen von $b$ als Verursacher des Lautwandels $o>\tilde{o}$ wie im Beispiel livO. ěbdô, ěobdi 'Silber' fi. hopea id. est. hõbe id. hält er dagegen für fraglich, da es seiner Meinung nach schwierig sei, die Formen

\footnotetext{
${ }^{13}$ Mit dem Übergangsdialekt Ii. meint PosTI (1942: XIV) den Übergangsdialekt des Dorfes Īra.
} 
zu erklären, die weiterhin $o$ beinhalten, obwohl ein $b$ im Auslaut der ersten Silbe existiert, z.B. liv. tobùD 'Krankheit'. Zwar macht auch Postı einige Bemerkungen zur Labialität und zu den Entsprechungen $o \sim \tilde{o}$, doch auch er kann, wie schon THOMSEN keine umfassende Antwort auf die Frage nach der Entwicklung dieser Entsprechung geben.

Ebenfalls von einem ursprünglichen $\tilde{o}$ geht BJÖRN COLLINDER (1960: 159ff.) in Comparative grammar of the Uralic Languages aus. Er sieht in seinen angeführten Beispielen eine klare Opposition von $*_{o} \sim * \tilde{o}$ im Finnougrischen und Uralischen bestätigt, weil er Beweise dafür im Samischen, Mordwinischen und Obugrischen sieht (ebd. 161). Der Großteil seiner Belege bezieht sich hier vor allem auf die beiden obugrischen Sprachen und deren Entsprechungen. Für das Mordwinische führt COLLINDER (ebd. 160) an, dass sich * $\tilde{o}$ in $e$-Stämmen zu $o$, in $a$-Stämmen dagegen zu $u$ entwickelt haben solle. Auffällig ist, dass er in seinen Überlegungen keine Angaben zu õ im Ostseefinnischen macht, sondern sich nur auf die eben erwähnten Sprachen bezieht und gelegentlich Hinweise auf das Finnische gibt. Auch bei seinen Betrachtungen von * õo verhält es sich ähnlich. Als ostseefinnische Entsprechungen führt er lediglich fi. uo und est. $o o$ aus Ururalisch $* \tilde{o}$ an (ebd. 161f.), liefert dabei aber keine genaue Beschreibung der Entwicklung.

Der These, dass $\tilde{o}$ primär sei, schließt sich auch ARNOLD KASK (1972: 123f.) an. Er beschreibt in seiner historischen Grammatik der estnischen Sprache (Eesti keele ajalooline grammatika) eine Aufteilung der ostseefinnischen Sprachen in die zwei bekannten Gruppen - die Südgruppe mit und die Nordgruppe ohne $\tilde{o}$ (bei ihm $\varrho$ ) - und dass der ursprüngliche Laut $\tilde{o}$ bewahrt wurde. Die Beispiele, die von KASK genannt werden, beinhalten im Finnischen allesamt ein $e$ in der ersten Silbe und einen Hintervokal in der Folgesilbe - ein Umstand, auf den er nicht hinweist, sodass offenbleibt, ob er eine Regelhaftigkeit der Beispiele erkannt hat: est. põrn 'Milz' fi. perna id.; est. võlg 'Schuld' fi. velka 'Geldschuld' oder auch est. tõru 'Eichel' fi. terho id. In diesem Punkt fügt er nichts zum bisherigen Kenntnisstand hinzu. Ferner behauptet KASK (ebd.), õ müsse es in Wörtern wie est. kollane 'gelb' und est. kord 'Mal', die im Wotischen und Südestnischen das $\tilde{o}$ bewahrt haben sollen, gegeben haben. Für den Grund seiner Annahme liefert er keine Erklärung. Es ist zu vermuten, dass KASK diese Wörter besonders hervorhebt, weil sie sich im Vergleich zu den anderen Beispielen, die er nennt, unerwartet anders verhalten: Ihre finnische Entsprechung beinhaltet in der ersten Silbe nämlich ein $e$, während die Folgesilbe 
hintervokalisch ist (fi. kelta 'gelb' zu est. kollane; fi. kerta 'Mal, Schicht' zu est. kord). Damit weichen sie deutlich von der Regel ab und benötigen eigentlich eine genauere Betrachtung. KASK (ebd. 124) erklärt, dass $\tilde{o}$ in den Inseldialekten zu $\ddot{o}$ geworden sei, während die Entsprechung in den nordestnischen Küstendialekten $e$ sei, seltener auch $o$. An dieser Stelle weist er außerdem ohne weitere Begründung darauf hin, dass die Möglichkeit bestehe, dass $\tilde{o}$ auch in Wörtern aufgetreten sei, die heutzutage im Estnischen ein $o$ in der ersten Silbe beinhalten, z.B. est. otsida 'suchen'. Auch er motiviert die Annahme, $\tilde{o}$ sei primär, in seinen Ausführungen nicht und liefert somit keine neuen Erkenntnisse in der Fragestellung nach dem Alter von $\tilde{o}$.

PAUl AlVRE (1976: 218) positioniert sich ebenfalls auf der Seite derer, die õ für einen ursprünglichen Laut halten. Er kritisiert die Annahme, dass $\tilde{o}$ sekundär sei, in seiner Rezension zu ARVO LAANESTS estnischer Fassung der Einführung in die ostseefinnischen Sprachen (Sissejuhatus läänemeresoome keeltesse) von 1975 und sagt, LAANEST vertrete damit „den Standpunkt der finnischen Sprachforscher, daß dieses $\underset{e}{e}$ in der ostseefinnischen Ursprache noch nicht als selbstständiges Phonem existierte“. ALvRE selbst bezeichnet Rekonstruktionen mit õ, wie z.B. *vēras 'Fremder', für „gebräuchlich“, liefert aber keine Argumente dafür, warum man $\tilde{o}$ als ursprünglich interpretieren sollte. Die reine Annahme einer Gebräuchlichkeit ist als Grundlage für eine fundierte Argumentation nicht ausreichend. Auf LAANEST und dessen Ausführungen wird an späterer Stelle noch eingegangen.

SEPPO SUHONEN (1988: 296) behauptet in seinem Beitrag Geschichte der ostseefinnischen Sprachen, dass es bei den Vokalen $e$ und $o$ eine sog. „wechselnde Vertretung“ gegeben haben müsse. Auch er nimmt an, dass es schon im Urfinnischen zwei unterschiedliche Vokale $e$ und $\tilde{o}$ (bei ihm e) gegeben habe. Dabei sei $e$ im Finnischen und Karelischen in allen Silben erhalten, im Estnischen, Wotischen und Livischen sei dagegen $\tilde{o}$ bewahrt geblieben. Letzteres sei zustande gekommen, weil es einen Lautwandel von $e>\tilde{o}$ in einem Teil der urfinnischen Dialekte gegeben habe, wenn die Folgesilbe hintervokalisch war (ebd.). Es handelt sich in seinen Augen also um ein dialektales Auseinanderentwickeln der beiden genannten Gruppen, bedingt durch die Annahme des Lautwandels. Für $o$ gibt SuHONEN (ebd.) an, dass es im Finnischen, Karelischen und Wepsischen regelmäßig erhalten sei, im Estnischen und Wotischen dagegen lediglich unregelmäßig. Die Bedingungen für die Entwicklung $o>\tilde{o}$ seien unbekannt. Es zeigt sich deutlich, dass auch SUHONEN sich vor allem auf die Lautentsprechung $e \sim \tilde{o}$ bezieht und den schwieriger zu begründenden Wandel $o>$ o nur ganz am Rande erwähnt. Alles in allem knüpft er damit 
an die zuvor diskutierten Beiträge an und liefert mit seinem Beitrag in der Fragestellung keine neuen Erkenntnisse.

PeTRI KALLIO hat sich 2014 in einem seiner aktuelleren Artikel namens The Diversification of Proto-Finnic ebenfalls der These angeschlossen, dass der Laut $\tilde{o}$ (bei ihm $\ddot{e}$ ) im Urostseefinnischen schon existiert haben müsse und greift so die ersten Ideen der Existenz eines $\tilde{o}$ in der ostseefinnischen Grundsprache wieder auf. Dabei lehnt er die heutzutage vorherrschende „finnozentrische“ Rekonstruktionsweise der ur(ostsee)finnischen Phonetik ab und nennt es viel mehr eine Innovation, dass die nördliche Dialektgruppe einen Wandel von $* \tilde{o}>* e$ durchlaufen haben solle, da die ostseefinnischen Dialekte seinerzeit viel diverser gewesen seien (KALLIO 2014: 160). Die Hauptaussage seiner Argumentation ist wie folgt (ebd. 161):

Although phonological innovations may of course diffuse across dialect boundaries, from a dialectological viewpoint $* \ddot{e}>* e$, occuring in only one tertiary branch of Finnic, should $a$ priori be considered more likely than $* e * \ddot{e}$, occuring everywhere else.

Einzuwenden ist, dass nicht nur ein Drittel der ostseefinnischen Sprachen keinen Laut $\tilde{o}$ besitzt, sondern über die Hälfte (Finnisch, Ingrisch, Karelisch und Wepsisch ${ }^{14}$ vs. Estnisch, Livisch und Wotisch) und dass dieses Mengenverhältnis mitsamt der Annahme einer größeren Diversität in den ostseefinnischen Sprachen bzw. Dialekten im Süden einen Lautwandel $e>\tilde{o}$ nicht alleine ausschließen können. Diese Diversität der Dialekte scheint KALLIO als Begründung jedoch auszureichen.

KALLIO (2014: 161) vermutet, dass *õ über Lehnwörter aus den baltischen oder germanischen Sprachen ins Ostseefinnische gekommen sein könne, auch wenn diese Sprachfamilien selbst kein $* \tilde{o}$ in der ersten Silbe kannten. Er begründet seine These damit, dass diese Sprachen vorwiegend $a$-Stämme besessen hätten und diese im Ostseefinnischen nicht zu den Vordervokalen der ersten Silbe im Hinblick auf die Vokalharmonie der uralischen Sprachen passten. Außerdem haben die Wörter mit * $\tilde{o}$ in der ersten Silbe seiner Meinung nach kaum Entsprechungen außerhalb des Ostseefinnischen (mit Ausnahme von *mëla 'Ruder', das unregelmäßig auf *melä id. zurückgehe). Auf welche Daten sich KALLIO bei seinen Hypothesen beruft, ist nicht erkennbar. Die wenigen Beispiele, die er anführt und rekonstruiert - für $\tilde{o}$ in der ersten Silbe sind es an dieser Stelle genau vier (ebd. 160) - stützen seine These nicht ausreichend, weil sie nur Fälle abdecken, in denen im Estnischen, Wotischen und Livischen gleichermaßen ein $\tilde{o}$ in der ersten

\footnotetext{
${ }^{14} \mathrm{Zu}$ den Bedingungen von $\tilde{o}$ im Wepsischen vgl. SUHONEN (1988: 296).
} 
Silbe anzutreffen ist. Nicht zu finden sind Beispiele für Wörter, in denen es in einer oder mehreren Sprachen (mit Ausnahme des Finnischen) einen anderen Vokal als $\tilde{o}$ in dieser Position gibt, z.B. fi. helppo 'leicht' est. hõlb 'Leichtigkeit' wot. helppo 'leicht' (liv. ohne Entsprechung) oder fi. terho 'Eichel' est. tõru id. wot. turu id. liv. tõ'rmõz id. Für germanische und baltische Lehnwörter nennt KALLIO in seiner Analyse gar keine Beispiele. Die Tatsache, dass es sich bei den Wortstämmen der germanischen Lehnwörter im Ostseefinnischen vorrangig um $a$-Stämme handelt, lässt sich bestätigen. Es gibt nur wenige Ausnahmen, bei denen der Ausgangspunkt der Entlehnung jedoch fraglich bleibt, z.B. fi. olut 'Bier' est. ôlu id. wot. elud id. liv. vo'l id. zu germ. *olut (LÄGLOS II: 310).

Einen Lautwandel $* o>\tilde{o}$ räumt KALLIO (2014: 161) zwar ein, bezeichnet ihn jedoch als ,sporadische Illabialisation“, die die Abnahme der Häufigkeit von $\tilde{o}$ in der ersten Silbe vom Wotischen über das Nordestnische, dann Südestnische bis hin zum Livischen erklären soll. Damit schließt er an KetTUNENS Annahme eines Kontinuums der Ausbreitung an.

Mit der Bezeichnung des Lautwandels $*_{o}>*^{*}$ als sporadisch stimmt KALLIO seinen Vorgängern zu und unternimmt keinen ernsthaften Versuch, die lautlichen Veränderungen zu erklären. Wodurch dieser vermeintlich sporadische Lautwandel ausgelöst worden sei, beschreibt KALLIO ebenfalls nicht.

\subsection{Das ostseefinnische $\tilde{o}$ als sekundäre Entwicklung}

Im Gegensatz zu den zuvor ausgeführten Hypothesen, wonach ein $\tilde{o}$ bereits in der Grundsprache vorhanden gewesen sein könnte, stehen die Positionen, gemäß derer dieser Laut eine sekundäre Entwicklung sei. Auch hier gibt es eine große Anzahl an Theorien, die ebenfalls bis an das Ende des 19. Jahrhunderts zurückreichen.

Abermals macht ARVID GENETZ (1897: 50) den Anfang, denn wie oben bereits erwähnt, revidiert er seine ursprüngliche Meinung zum Alter von o 20 Jahre nach seiner karelischen Lautlehre in einem Beitrag zu den Vokalen der ersten Silbe im Finnischen, Samischen und Mordwinischen und weicht von seiner These, der Vokal habe in der Grundsprache bereits vorgelegen, wieder ab. Dieser Beitrag stellt somit die erste Position dar, die eine klare Aussage zu $\tilde{o}$ als sekundäre Erscheinung beinhaltet, auch wenn dort von GENETZ ebenfalls keine konkreten Begründungen für sein Umschwenken angegeben oder Herleitungsversuche gemacht werden. 
Lange war die Annahme, õ sei primär, dominant, sodass die nächste Gegenposition dazu erst gut 50 Jahre nach GENETZ zu finden ist: WoLfGANG STEINITZ (1944: 117) hält es für unrealistisch, „dass osfi. $\underset{e}{\text { und }} *_{i} \underset{i}{ }$ Fortsetzungen des hinteren illabialen fiugr. $*_{i}$ sind“. Er interpretiert diese Vokale als spät, vermutet aber, dass vielleicht einige Wurzeln in die ostseefinnisch-mordwinische Zeit zurückreichen könnten. Mehr hat er zu diesem Thema nicht zu ergänzen, sondern er verweist dazu auf LAURI HAKULINEN und ERKKI ITKONEN.

HAKULINEN $^{15}$ (1979: 37f.) erklärt, dass sich $\tilde{o}$ (hier $e$ ) aus $e$ in hintervokalischen Wörtern durch Assimilation entwickelt haben müsse. Belegbar sieht er dies durch baltische und germanische Lehnwörter. Im Gegensatz zu KALLIO (s. o.) später sieht HAKULINEN jedoch kein ursprüngliches $\tilde{o}$, sondern geht bei diesem Laut von einer sekundären Entwicklung aus. HAKULINEN deutet also als erster auf eine Verbindung zwischen der Entwicklung von $\tilde{o}$ und baltischen und germanischen Lehnwörtern hin. Er ist zudem der erste, der den Zusammenhang zwischen diesem Lautwandel und dem Hintervokal in der Folgesilbe als absolute Bedingung herstellt, auch wenn er nicht der erste ist, der Hintervokale als in diesem Zusammenhang ausschlaggebend erkennt (s. o. KetTUNEN). Er führt seine Annahme jedoch an dieser Stelle nicht weiter aus und es ist nicht erkennbar, auf welche Datenlage er sich hier beruft.

Auf ERKKI ITKONEN wird in der einschlägigen Literatur nicht nur von STEINITZ, sondern auch von diversen anderen Wissenschaftlern immer wieder verwiesen und somit gehört sein zu Anfang bereits zitierter Artikel zu einem der zentralen in dieser Thematik. ITKONEN (1945: 159) stellt darin nicht nur die frühen Anfänge der Erforschung von õ zusammen, sondern beschäftigt sich auch mit der Entwicklung des Vokalismus des Ostseefinnischen im Hinblick auf die Frage, ob die Möglichkeit bestand, dass es dort schon früh eine Mittelvokalreihe gegeben haben könne. Dabei beschränkt sich ITKONEN nicht nur auf eine Analyse des ostseefinnischen Lautstandes und der bisher aufgestellten Theorien, denn er bezieht auch das Marische, das Mordwinische, das Samische und die obugrischen Sprachen mit ein, um seine Thesen zu begründen. Insbesondere die oben bereits diskutierte These E. N. SETÄLÄs, dass es verschiedene Laute $i$ und $i$ bzw. $e$ und $e$ gegeben haben müsse, überprüft er genau und kommt zu der Schlussfolgerung, dass dessen Annahme falsch sei, da $\tilde{o}$ im Ostseefinnischen eindeutig eine sekundäre Entwicklung

\footnotetext{
${ }^{15}$ STEINITZ (1944: 117) bezieht sich auf die erste Auflage von HAKULINENS Suomen Kielen Rakenne ja Kehitys (Struktur und Entwicklung der finnischen Sprache) von 1941. Die hier angebrachten Verweise sind der überarbeiteten vierten Auflage von 1979 entnommen.
} 
sein müsse (ebd. 178). Die nach ITKONENS Aussage für SETÄLÄ angeblich wichtige Annahme, nämlich dass es im Wotischen Wörter mit $i$ in der ersten Silbe und $e$ in der zweiten gibt, z.B. wot. sie 'Verbindung', und dies auf eine frühere Existenz von $i \underline{i}$ an der Stelle von $i$ hinweisen soll, entkräftet ITKONEN (ebd. 170ff.) beispielsweise später dadurch, dass er es zu einem Fall von Verallgemeinerung durch Analogie erklärt. ${ }^{16}$

Zwei Dinge setzt ITKONEN (ebd. 159) zu Beginn seiner Analyse voraus, um die lautlichen Verhältnisse im Ostseefinnischen überhaupt klären zu können: 1.) Die Vokalharmonie muss es bereits im Spätostseefinnischen, also der ostseefinnischen Grundsprache, gegeben haben und 2.) hinsichtlich dieser Vokalharmonie seien die Laute $e$ und $i$ neutral, in velarer Umgebung leicht velarisiert gewesen, also ę und $i$.

In seiner Betrachtung widmet sich ITKONEN (ebd. 161f.) zunächst der Lage des Vokalismus im Samischen und erklärt, dass die Entwicklung hier deutlich sei. So habe es in der ersten Silbe in der Entwicklung eine Abfolge $e>\varepsilon>a$ gegeben. Daher sind $e$ und $\underline{i}$ lediglich als Zwischenstufe anzusehen, jedoch nicht als ursprünglicher Laut. Als Beweis dafür sind für ihn u. a. fi. mela 'Ruder' est. mõla id. lp. malle id. oder fi. neuvo 'Rat, Ratschlag' est. nõu id. 1p. næevvo id. eindeutige Beispiele.

Im Anschluss daran befasst sich ITKONEN (ebd. 162ff.) mit den lautlichen Verhältnissen im Mordwinischen. Er setzt auch in dieser Sprache im Vokalismus Hinweise auf eine ursprüngliche Vokalharmonie voraus und hält fest, dass die Entsprechungen für fi. $e, i$ im Mordwinischen vordervokalisch seien, sofern in der Folgesilbe $e$ oder $\ddot{a}$ vorlagen. Auch Abweichungen davon erklärt er, sowie die für das $\tilde{o}$ der ersten Silbe nicht relevante Reduktion des $e$ in der zweiten Wortsilbe. Alles zusammengenommen kommt ITKONEN zu der Schlussfolgerung, dass es im Mordwinischen keine Mittelvokalreihe gegeben haben könne. Gleiches gelte auch für das Marische, mit dem er sich im Anschluss an das Mordwinische auseinandersetzt (ebd. 165ff.). Dort sei die Entsprechung für fi. $e, i(\bar{e}, \vec{\imath})$ in vordervokalischen Stämmen ein palataler Vokal (mit Ausnahme des Ostdialektes, in dem dann ein $\partial$ vorliegt, wenn in der marischen Ursprache $e$ und $i$ reduziert gewesen seien). In Wörtern, die einen ursprünglichen Hintervokal in der zweiten Silbe haben, gebe es eine doppelte Vertretung von $e$ und $i$, denn ein Teil der Beispiele sei vor-

\footnotetext{
${ }^{16}$ Unklar ist, auf welche Quelle sich ITKONEN in seinem Artikel genau beruft. Die Verweise auf das sog. Iso Tietosanakirja haben sich als falsch herausgestellt, da er sich anscheinend auf eine andere Veröffentlichung, nämlich das Tietosanakirja (CASTRÉN [et al.] (1917); CASTRÉN [et al.] (1919)), bezieht. Allerdings führt SETÄLÄ seine Theorien dort nicht aus und postuliert lediglich die Existenz von hinterem und vorderem $i$ und $e$ in der Grundsprache. Daher muss an dieser Stelle auf ITKONENS Ausführungen zurückgegriffen werden.
} 
der-, der andere hintervokalisch (ebd. 165). Für das Marische führt ITKONEN (ebd. 166) abermals das lautliche Verhältnis für die zweite Silbe an, um das Bild des Vokalismus zu vervollständigen. Das Zwischenfazit, das ITKONEN aus seiner bisher dargelegten Analyse zieht, ist, dass SETÄLÄS Theorie nicht korrekt sein kann, weil das sprachliche Material diese Hypothese nicht bestätigt. Das Mordwinische und das Marische zeigen seiner Meinung nach eindeutig, dass $e$ und $i$ in diesen beiden Sprachen niemals Mittelvokale oder gar Hintervokale gewesen sein können (ebd. 167).

An die Betrachtung der wolgafinnischen Sprachen anknüpfend folgt schließlich ITKONENS ausführliche Analyse des ostseefinnischen Vokalismus. Dabei stellt er bei einer Annahme von $e$ und $i$ für die Grundsprache die Wahrscheinlichkeit infrage, dass $i$ und $i$ zusammengefallen sein könnten, während sowohl $e$ als auch $e$ bewahrt wurden. Viel logischer erscheint seine Theorie, von velarisierten Vokalen (s. o.) auszugehen, da für das Zusammenfallen von $i$ und $i$ ein vollständiger Lautwandel notwendig gewesen wäre, während ein Zusammenfallen von $e$ und $e$ einfach vor sich gehen konnte (ebd. 169f.). Das laut ITKONEN für SETÄLÄ wichtigste wotische Beispiel, das die Annahme von $*_{i}^{i}$ belegen soll, nämlich wot. sie 'Verbindung' erklärt ITKONEN (ebd. 170f.) über eine sekundäre Velarisierung. Fälle wie dieser sollen sich erst dann entwickelt

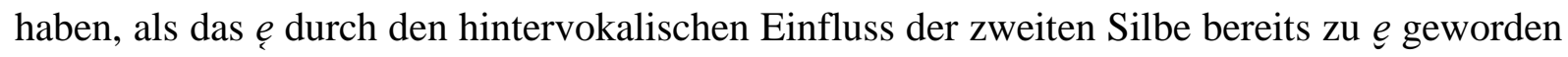
sei. Der Umstand, dass alle Stämme mit $e-e$ vordervokalisch seien, ist für ihn ein deutlicher Beweis dafür, dass sich $\varrho$ erst später herausgebildet haben könne (ebd. 170). Auch scheinen Parallelformen oder Ableitungen, die hintervokalisch sind, auf das Wort Einfluss genommen zu haben, wodurch ein Lautwandel $e>e$ ausgelöst worden sei, z.B. estS. hikõ 'Schweiß-PartSg', vgl. est. (dial.) higone, higonema estS. hiGònęs 'schwitzen-3SGINDPRÄS' (ebd. 171f.). ITKONEN (ebd. 174f.) zufolge sei auch die späte Entstehung von $\ddot{o}$ ein Beweis seiner Theorie, denn wenn $e$ und $i$ keine neutralen Vokale gewesen wären, wäre es gar nicht erst zu der Herausbildung eines neuen Lautes $\ddot{o}$ gekommen. Einen weiteren Beleg seiner These sieht ITKONEN (ebd. 175f.) darin, dass die vordervokalische Variante des suffixalen $u$, nämlich $\ddot{u}$, nur nach $\ddot{a}$, $\ddot{u}$ und $\ddot{o}$ in der vorhergehenden Silbe vorliegt. Nach $e$ und $i$ folge meist $u$. Daraus schlussfolgert er, dass es sicher sei, dass im Urostseefinnischen ein Hintervokal nicht durch einen Lautwandel zu einem Vordervokal geworden sein könne. Die beiden Vokale $e$ und $i$ müssen hinsichtlich der Vokalharmonie neutral gewesen sein, da es eine Anpassung der hintervokalischen Ableitungssuffixe an vordervokalische Stämme schon in der spätostseefinnischen Zeit gegeben haben müsse. Ausnahmen von dieser Regel bilden einzig und allein die Wörter fi. mer-ta 'Meer-PARTSG' und ver-ta 'Blut-PARTSG' (ebd. 178). 
ITKONEN (ebd. 178f.) kommt am Ende zu der Schlussfolgerung, dass sich das $e$ in den ostseefinnischen Sprachen erst später durch eine hintervokalische Umgebung aus einem leicht velarisierten $e$ herausgebildet haben könne. Den Lautwandel $o>e$ bezeichnet er als unregelmäßig und er formuliert als erster deutlich, dass dieser im Estnischen und Wotischen stattgefunden hat, während das Livische davon kaum betroffen ist (ebd. 181f.). Diese Erkenntnis ist für die Analyse des Vokalismus der ostseefinnischen Sprachen wichtig, da unbedingt der Frage nachgegangen werden muss, welche Gründe es dafür geben könnte, warum ausgerechnet das Livische in diesem Lautwandel so stark von den anderen $\tilde{o}$-Sprachen abweicht. ITKONEN (ebd. 182) hält für möglich, dass es auch eine Rolle spielt, dass $o$ der Hintervokal war, der dem neu entstandenen $e$ rein lautlich gesehen am nächsten war. Daher habe $o$ in Richtung des neuen Vokals schwanken können und dieser hatte daraufhin die Möglichkeit, nach und nach einen Lautwandel zu bewirken. Dieses Phänomen sei ITKONEN (ebd.) zufolge auch im Vermischen der Artikulation von $e$ und $o$ in einigen Wörtern wie z.B. est. kord 'Mal' oder est. hobune 'Pferd' zu erkennen. Die genannten Wörter sind allesamt hintervokalisch und entsprechen in ihrer Form denen, für die KASK (s. o.) zuvor die Behauptung aufgestellt hat, dass in ihrer ersten Silbe früher $\tilde{o}$ vorgelegen haben müsse.

ITKONENS Beitrag löste einen wissenschaftlichen Streit zwischen ihm und KETTUNEN aus. Letzterer schrieb den Artikel Eikö kantasuomessa ole ollut keskikielen vokaaleja? (Hat es im Urostseefinnischen keine Mittelvokale gegeben?) (KETTUNEN 1948) als Antwort auf ITKONENS vorausgegangene Analyse und hielt darin an seinen oben bereits genannten Positionen fest. KETTUNEN diskutiert dabei ITKONENS Artikel und sieht die finnische Vokalharmonie als Beleg dafür, dass es ein hinteres $e$ parallel zum vorderen $e$ gegeben haben müsse, weil es nämlich Wörter wie fi. velka 'Schuld' und fi. mela 'Ruder' gibt, aber auch Beispiele wie fi. sepä 'hochgebogener Vorderteil des Schlittens' und fi. kesä 'Sommer'. Letztere haben sich ihm zufolge aus den Wörtern mit urostseefinnischem vorderen $e$ in der ersten Silbe entwickelt. Beispiele mit Hinter- und Vordervokalen finden sich auch in Wörtern mit $i$ in der ersten Silbe und dieses soll auf ein hinteres $i$ hingewiesen haben. Irgendwann habe es dann eine Entwicklung zu einem vorderen $i$ gegeben, ganz wie es auch bei $e$ gewesen sein soll (ebd. 120). Zudem bezeichnet er ITKONENS Darstellung als einen „künstlichen Erklärungsversuch“ (ebd. 121). KETTUNEN kommentiert und analysiert dessen Artikel mit all seinen Aspekten und kommt zu dem Ergebnis, dass ITKONEN 
keinen bedeutsamen Beitrag geleistet habe. Insgesamt liefert er mit dieser Bewertung des Artikels in der Diskussion um das Alter von $\tilde{o}$ aber keine neuen Erkenntnisse oder Argumente für bzw. gegen eine der Positionen.

GYULA DÉCSY (1965: 155) erklärt in Einführung in die finnisch-ugrische Sprachwissenschaft, dass es in der gemeinsamen finnisch-ugrischen Grundsprache nur sechs Vokale gegeben haben könne und dass weitere Vokale, so auch $\tilde{o}$, für diese Sprachstufe nicht vorausgesetzt werden können. DÉCSY ist der Meinung, dass es für eine Annahme von u. a. o für die Grundsprache keine ausreichende Begründung gebe und spricht sich gegen die Theorien anderer, beispielsweise die von ColLINDER (s. o.), aus. Auch andere Vokale, die von einigen Forschern als ursprünglich angenommen wurden, hält er für diese Sprachstufe für unwahrscheinlich, z.B. $\ddot{o}$ oder $\ddot{u}$ (ebd.). Seiner Meinung nach bildeten lediglich die Vokale $a, e, i, o, u$ und $\ddot{a}$ den Vokalismus der Grundsprache, wobei nur $a$, $e$ und $\ddot{a}$ Bestandteil von nichtersten Silben sein konnten (ebd. 155). DÉCSY fasst seine Ansichten recht kurz und trägt zu der eigentlichen Diskussion nicht mehr bei, als dass er die Theorie eines primären $\tilde{o}$ ablehnt.

Im Jahr 1978 veröffentlichte TIIT-REIN VIITSO zwei nahezu identische Artikel, die sich konkreter mit der Herkunft des ostseefinnischen $\tilde{o}$ in der ersten Silbe befassen: Läänemeresoome esimesi silbi õ ajalugu (Die Geschichte des ostseefinnischen $\tilde{o}$ in der ersten Silbe) und The history of Finnic o in the first syllable. Er beschreibt darin, dass es in einigen ostseefinnischen Sprachen das Phonem õ gibt bzw. gab und dass dieses mitunter geschwunden sei, während es in anderen Sprachen dieses Sprachzweigs nie ein Phonem õ gegeben habe bzw. dessen Schwund dort bisher nie nachgewiesen werden konnte (VIITSO 1978a: 81; VIITSO 1978b: 86). Dabei vergleicht VIITSO als erster mehr als nur zwei Sprachen miteinander und kann so einen besseren Überblick über das Ausmaß des Lautwandels geben. Von ihm in die Betrachtung mit einbezogen werden eine Vielzahl an Beispielen aus dem Estnischen, Wotischen, Livischen und Südestnischen, sowie dem Finnischen als Vergleichsgröße. VIITSO unterteilt die Sprachen, in denen das Phonem vorkommt, im weiteren Verlauf in insgesamt fünf verschiedene Dialektgruppen und versucht anhand derer die Herkunft und Entwicklung von $\tilde{o}$ zu analysieren. Die Annahme einer Ursprünglichkeit von $\tilde{o}$ sei ihm zufolge nicht realistisch, da beispielsweise der Umstand, dass $\tilde{o}$ als einziger Vokal nicht am Ende eines einsilbigen urostseefinnischen Stammes aufgetreten sei, dagegen spreche (VIITSO 1978a: 85f.; VIITSO 1978b: 89). Er ist der erste, der explizit auf diesen Umstand hinweist: 
First, * $\tilde{o}$ is the single vowel, that did not occur at the end of a monosyllabic root in ProtoFinnic. Likewise it never occurs in this position in Livonian, Ugala, Estonian and Votian except in the name of the letter $\tilde{o}$. Second, most of the stems with $* \tilde{o}$ are clearly loans or have no etymological counterparts in cognate languages. For loans, the equivalent of * $\tilde{o}$ is normally $e$, rarely $i, i e, i a$. (VIITSO 1978b: 89)

Zudem verweist er auf ERKKI ITKONEN (s. o.) und bestätigt dessen Annahme, dass man in den Wörtern mit Entsprechungen in den verwandten Sprachen keinen Beweis für ein historisches $*^{*} \tilde{o}$ finde. Wie dieser bereits angeführt hatte, sei die samische Entsprechung zu $\tilde{o}$ der Laut ${ }_{\varepsilon}$, im Mordwinischen gehen die Entsprechungen etwas auseinander. VIITSO (1978b: 89f.) führt ebenfalls an, dass man von einer Entwicklung $* e>\tilde{o}$ in hintervokalischen Stämmen ausgehen solle, auch wenn zu bedenken sei, dass nicht klar sei, ob *e überhaupt in der ersten Silbe solcher Stämme auftreten konnte. Er ist der Meinung, dass „,back-vowel genuine stems with $e$ in the first syllable were formed in the course of the development and splitting of Proto-Finnic on the basis of front-vowel roots and perhaps partly from back-vowel roots. In the latter case a backvowel or *i became * $e "($ ebd. 90$)$.

Im weiteren Verlauf rekonstruiert er eine mögliche Entwicklung der Laute im Ostseefinnischen und kommt dabei schließlich auf insgesamt sieben Sprachstufen, die er anhand der dargelegten Lautwandel für realistisch hält. Die Lautwandel $*_{e}>\tilde{o}$ und $*_{o}>\tilde{o}$ nimmt er dabei erst für die vierte Sprachstufe an, also die Dialektgruppe bestehend aus Wotisch, Estnisch, Südestnisch und Livisch (ebd. 92, 104). VIITSO (ebd. 104) sieht in seiner eigenen Analyse auch ITKONENS oben bereits erläuterte Theorie zur Entwicklung von $\tilde{o}$ bestätigt.

BJÖRN COLLINDERS oben bereits diskutierte Analysen zum uralischen und finnougrischen Vokalismus wurden von PEKKA SAMMALLAHTI Ende der 1970er für überholt gehalten, denn jener „,basiert seine Auffassung über den Vokalismus hauptsächlich auf die Lautverhältnisse im (Ostsee)-finnischen, Lappischen und Mordwinischen“ (SAMMALlaHTI 1979: 23). Die Sprachgeschichte zu den samojedischen Sprachen sei erst nach dem Erscheinen von CoLLINDERS Monografien erweitert worden (ebd. 24), sodass SAMMALLAHTI bereits ausführlichere Daten auswerten konnte. SAMMALLAHTI selbst nimmt ein von ITKONEN festgelegtes Schema für die Laute der finnougrischen Grundsprache als Grundlage für seine eigene Theorie. ${ }^{17}$ Dieses Schema besagt, dass die Grundsprache im Lautstand und in ihrer Struktur mehr oder weniger genau dem

\footnotetext{
${ }^{17}$ Die angenommene Rekonstruktion des samojedischen Lautstands stützt SAMMALLAHTI (1979: 24) vor allem auf die Arbeit von JUHA JANHUNEN (1975-1976). Im Phonemparadigma der samojedischen Grundsprache nennt er dort insgesamt elf Vokale. Neben $*_{i}^{i}$ und $*_{e}$ nimmt er noch die Laute $* e, *_{i}{ }^{*} o, * u, * \ddot{a}, * \ddot{o}, * \ddot{u}, * a ̊$ und $* \hat{a}$ an.
} 
Stand des Frühurfinnischen entspreche und dass im Gegensatz zu der Grundsprache der samojedischen Sprachen dort kein $\tilde{o}$ (bei ihm e) vorgelegen habe (ebd. 23f.). Zwar geht SAMMALLAHTI (ebd. 57ff.) in der uralischen Grundsprache wegen der ursamojedischen Laute $\underline{i}$ und $e$ von der möglichen Existenz eines $*_{i}$ aus, doch von diesem gibt es im Finnougrischen keine Fortsetzer, da es dort schließlich nach der Aufspaltung der uralischen Grundsprache in die finnougrische und die samojedische Grundsprache mit * $a$ zusammengefallen sei.

ARVO LAANEST (1982: 127) vertritt in Einführung in die ostseefinnischen Sprachen ${ }^{18}$ ebenfalls die Meinung, dass $\tilde{o}$ (bei ihm $e$ ) nicht als eigenständiges Phonem im Urostseefinnischen existiert habe, auch wenn es möglich sei, dass es in hintervokalischen Wörtern eine Variante von $e$ gegeben haben könne. Ihm zufolge entspreche $\tilde{o}$ in den Sprachen ohne diesen Laut meist $e$. Er erwähnt zudem, dass aus einigen wepsischen Dialekten ebenfalls $\tilde{o}$ bekannt sei, sagt aber auch, dass dessen Bedingungen nicht mit denen in den anderen ostseefinnischen Sprachen vergleichbar seien (ebd. 128). ${ }^{19}$ LAANEST stellt selbst keine Regeln für die Bedingungen der Lautwandel zu $\tilde{o}$ auf. Er erkennt zwar, dass es sie gegeben haben muss, spricht aber nur von einem „unre-

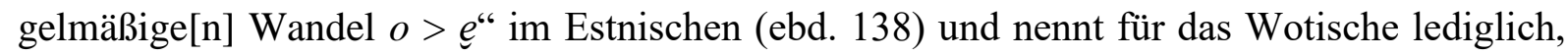
dass es einen Wandel von $o>\tilde{o}$ in den Wörtern gegeben haben müsse, in denen das Estnische $o$ schreibt (ebd. 137). Umfassende Daten analysiert er augenscheinlich in seiner Arbeit nicht, sodass nur von Bemerkungen die Rede sein kann. Festzuhalten ist, dass LAANEST in seiner Monografie keine neuen Erkenntnisse in der Frage nach dem Alter von õ beisteuern kann und auch keine konkreten Regeln oder Bedingungen für seine Beispiele benennt.

Eine längere Ausführung zur Thematik des õ im Estnischen liefert KALEVI WIIK (1986) in seiner Monografie Viron õ (Das estnische o). Dort untersucht er den Laut aus unterschiedlichen Blickwinkeln, u. a. artikulatorischen und akustischen, aber auch im Hinblick auf die estnischen Dialekte und die Entsprechungen zwischen dem Finnischen und dem Estnischen (ebd. 5). Er bezieht sich dabei immer wieder darauf, dass er finnischen Muttersprachlern durch Regeln für die Lautentsprechungen deutlich machen möchte, wann und unter welchen Bedingungen die finnischen Laute im Estnischen zu $\tilde{o}$ werden und wann nicht.

\footnotetext{
${ }^{18}$ Der Originaltitel Sissejuhatus läänemeresoome keeltesse erschien bereits 1975. In dieser Arbeit wird auf die deutsche Ausgabe von 1982 Bezug genommen.

${ }^{19}$ Näheres zu den Bedingungen lässt sich bei SUHONEN (1988: 296) finden. Dieser sagt, dass $e$ in einem Teil des Wepsischen ,nach einem nichtpalatalisierten Konsonanten zu dem Hintervokal ę geworden ist“".
} 
Für das estnische õ nennt WIIK (ebd. 37) insgesamt sechs finnische Vokale als mögliche Entsprechung, nämlich $o, e, a, u, \ddot{o}$ und $i$. In seinem Material entfällt der Großteil der Entsprechungen (55\%) auf fi. $o$, ein weiterer beachtlicher Teil (32\%) auf $e$ (ebd.). WIIK (ebd. 40) stellt fest, dass $\tilde{o}$ in der ersten Silbe im Finnischen $e$ entspricht, wenn das Wort hintervokalisch ist, est. $e$, wenn keine Hintervokale vorliegen, z.B. fi. veri 'Blut' est. veri id., fi. velka 'Schuld' est. $v \tilde{o l g}$, gen. - $a$ id. Damit knüpft er erwartungsgemäß an vorausgegangene Analysen und Interpretationen anderer Wissenschaftler an. Zudem erklärt WIIK (ebd. 40f.) beispielsweise, welche Lautwandel aus historischer Perspektive bei einer Deutung der Daten relevant seien (z.B. *ee $>i e$ ) und dass Lehnwörter nicht unbedingt als Teil der $e$-Entsprechungen behandelt werden müssen.

Auch fi. $o$ als ursprünglicher Vokal wird von WIIK (ebd. 42ff.) untersucht. Dabei stellt er fest, dass es keine eindeutige Regel dafür gibt, wann fi. $o$ zu est. $o$ und wann zu est. $\tilde{o}$ wird (ebd. 42). $\mathrm{Zu}$ bemerken sei auch, dass sich fi. uo und est. $\tilde{o} \tilde{o}$ nie entsprechen. Abermals ist erkennbar, dass in dieser Arbeit vor allem die Entsprechungen $e \sim \tilde{o}$ erklärt werden, während Erklärungsversuche zu $o \sim \tilde{o}$ nur auf Theorien basieren.

In seiner Analyse stellt er ebenfalls fest, dass es einige Wörter im Estnischen gibt, in denen eigentlich ein $\tilde{o}$ zu erwarten wäre, weil sie hintervokalisch sind. Dass dort dennoch ein $o \mathrm{zu}$ finden ist, erklärt WIIK (ebd. 41) dadurch, dass es eine Entwicklung $e>\tilde{o}>o$ gegeben haben könne. Beispiele, die er nennt, sind est. hobune 'Pferd' fi. hevonen id., est. kord 'Ordnung, Reihe' fi. kerta 'Mal, Schicht' und est. kollane 'gelb' fi. keltainen id. Die Beispiele sind - mit Ausnahme von est. hobune - bereits von KASK und ITKONEN (s. o.) bekannt. WIIK liefert im Gegensatz zu KASK einen Erklärungsversuch und geht davon aus, dass $\tilde{o}$ erst eine spätere Stufe der lautlichen Entwicklung war.

Für die Diphthonge ou und oi postuliert WIIK (ebd. 43) ebenfalls zwei Regeln für den Lautwandel: Für den finnischen Diphthong ou gelte seiner Analyse zufolge, dass dessen erster Bestandteil, also $o$, im Estnischen grundsätzlich immer $\tilde{o}$ entspreche, z.B. fi. soutaa 'rudern' est. sõuda id. Für fi. oi (und $\ddot{o} i$ ) gelte, dass der erste Bestandteil immer dann est. $\tilde{o}$ entspreche, wenn das $i$ des Diphthongs der Imperfektmarker ist, also beispielsweise in Verben wie fi. juoda 'trinken' oder fi. luoda 'erschaffen', die im estnischen Imperfekt (hier 1.Sg.) zu jõin und lõin werden (ebd. 43). WIIK (ebd. 44) selbst schreibt zu den Fällen, die nicht zu den oben genannten Erklärungen passen, zusammenfassend nur, dass fi. $o$ im Estnischen mal $\tilde{o}$, mal $o$ entspricht. Für finnische Laute, die nicht $e$ oder $o$ sind (also beispielsweise $a$ oder $i$ in Wörtern wie fi. sana 'Wort' oder fi. sisar 'Schwester') und im Estnischen õ entsprechen, hat WIIK (ebd. 44f.) keine 
Erklärung. Dass man gewisse grammatikalische Bedingungen und einen Teil der Lehnwörter nicht mit in die Untersuchung der Lautwandel einbeziehen kann, erkennt er richtig (ebd. 45). Zusammenfassend lässt sich zu WIIK sagen, dass er zur eigentlichen Thematik mit seiner Monografie kaum etwas Neues beiträgt. Seine Betrachtungen und Resultate zur Entsprechung fi. $e$ $\sim$ est. $\tilde{o}$ decken sich mit den bisherigen Ergebnissen anderer Wissenschaftler und auch zu fi. $o$ est. $\tilde{o}$ kann er keine frischen Erkenntnisse beisteuern. Wichtig anzumerken ist jedoch, dass sich WIIK als erster explizit mit den Diphthongen, die von diesen lautlichen Veränderungen betroffen sind, auseinandersetzt. Er betrachtet sie sowohl von $e$, als auch von $o$ ausgehend, bezieht historische Lautwandel in seine Analyse mit ein und versucht sie in die bereits vorhandenen Regeln zu den Bedingungen dieser lautlichen Veränderungen einzubinden.

Ein weiterer, etwas jüngerer Artikel, der die Problematik der Frage nach dem Alter von $\tilde{o}$ aufgreift, ist der des allgemeinen Sprachwissenschaftlers JAN HENRIK HOLST. Er versucht in Die Herkunft des estnischen Vokals õ (HOLST 2001) durch einen direkten Vergleich zwischen dem Finnischen und dem Estnischen den Ursprung von $\tilde{o}$ zumindest im Hinblick auf das Estnische zu erklären. Diese Gegenüberstellung unternimmt er mittels eines Wortvergleichs in etwas gröBerem Umfang. ${ }^{20}$ HoLST ist ein Vertreter der These, dass es $\tilde{o}$ in der ostseefinnischen Grundsprache noch nicht gegeben habe und dass dessen Vokalismus dem des heutigen Finnischen, entsprochen haben müsse. Erst verschiedene Lautwandel sollen den Laut $\tilde{o}$ verursacht haben (ebd. 95). So entspreche finnisches $e$ im Estnischen $\tilde{o}$, wenn die Folgesilbe hintervokalisch war, und einem $e$, wenn nicht. Dem finnischen $o$ entspreche ein estnisches $\tilde{o}$, wenn die Folgesilbe vordervokalisch war oder keine vorliegt, und einem $o$, wenn nicht. Diese Annahmen sind keine von HOLST neu gewonnene Erkenntnis, denn sie knüpfen ganz offensichtlich direkt an die Arbeiten von u. a. WIIK und VIITSO (s. o.) an. Gleichzeitig erkennt Holst (ebd. 78), dass der zweite Teil dieser These, also die Entsprechungen $o \sim \tilde{o}$, sehr viele Ausnahmen aufweist, die er im Laufe seines Aufsatzes durch verschiedene Mittel zu erklären versucht. Aus diesem Grund postuliert er zunächst ein sogenanntes Labialitätsgesetz, das besagt, dass im Estnischen ein kurzes $o$ in direkter Umgebung eines labialen Lautes zu $\tilde{o}$ wurde und erklärt so einen beachtlichen Teil der Abweichungen von seiner aufgestellten Regel (ebd. 79f.):

Das Estnische hat in einer frühen Zeit seines Sonderlebens eine Phase durchgemacht, in der seine Phonotaktik kein kurzes $o$ in unmittelbarer Nachbarschaft eines $p, m, v$ oder $u$

\footnotetext{
${ }^{20}$ HOLST (2001: 78) macht seine Analyse mit insgesamt 300 Entsprechungen, bei denen knapp über die Hälfte aus Kurzvokalen besteht. Ein Bestandteil dieser Entsprechungen sind auch Beispiele ganz ohne õ als Vergleichsgegenstand. Dabei handelt es sich um insgesamt 165 Wortpaare (ebd. 77).
} 
duldete. Es gab also kein ${ }^{\mathrm{x}} p o,{ }^{\mathrm{x}} o p,{ }^{\mathrm{x}} m o,{ }^{\mathrm{x}} o m,{ }^{\mathrm{x}} v o,{ }^{\mathrm{x}} o v$ und ${ }^{\mathrm{x}} o u$. (Ein ${ }^{\mathrm{x}} u o$ hat es sowieso nicht gegeben.) Dieser Zug ist bisher nicht dokumentiert gewesen. Bei $p, m, v$ und $u$ handelte es sich um alle Laute des Estnischen, die etwas mit Labialität zu tun hatten, mit Ausnahme des $o$ selbst (das nicht vor oder hinter sich selbst stehen kann, es sei denn, man käme auf die Idee, den langen Vokal oo so aufzufassen) und der beiden vorderen gerundeten Vokale $\ddot{u}$ und $\ddot{o}$ (neben denen wegen der Vokalharmonie nie $o$ stand). [...] Schon lange besteht die phonotaktische Eigenart nicht mehr; dies liegt an onomatopoetischen Wörtern und Entlehnungen jeglicher Art. ${ }^{21}$

Zwar ist HOLST nicht der erste, der einen Zusammenhang zwischen $\tilde{o}$ und der Labialität der lautlichen Umgebung herstellt - man erinnere sich dabei an THOMSEN, der bereits 1890 eine Verbindung von $v$ und $\tilde{o}$ im Livischen sieht und auch PosTI, der auf $b$ als labialen Laut verweist - er ist jedoch der erste, der einen Zusammenhang derart umfassend formuliert.

Andere Abweichungen, die nicht unter das Labialitätsgesetz fallen, erklärt HoLST (ebd. 81-86) als Lehnwörter, Analogiefälle und Onomatopoetika. Hinzu kommen bei ihm Wörter der Kategorie „Sonstiges“, bei denen sich zeigen soll, dass ,,in Wirklichkeit nichts Unregelmäßiges in der Lautentwicklung vorliegt; es erweckt lediglich die Komplexität der Entwicklung zunächst den Anschein“ (ebd. 86). Seine Erklärungen dazu wirken mitunter wenig nachvollziehbar. So behauptet er beispielsweise, dass das Paar fi. sota 'Krieg' est. sõda id. im Estnischen ein Lehnwort aus einer anderen ostseefinnischen Sprache sein muss, in der $*_{o}>\tilde{o}$ regelmäßig sei. Diese sind in seinen Augen das Wotische und „,bestimmte estnische Dialekte“ (ebd. 88). ${ }^{22}$ Wodurch diese angenommene Regelmäßigkeit gegeben sein soll, erklärt HOLST nicht. Er selbst kann diese wohl kaum bestätigen, da sich sein Forschungsmaterial ausschließlich auf das Finnische und das Estnische beschränkt und nicht erkennbar ist, ob er sich überhaupt näher mit dem Wotischen oder einer der anderen ostseefinnischen Sprachen befasst hat. Es finden sich in seiner Arbeit noch weitere Beispiele, die nicht stimmig erklärt sind: HOLST zufolge sei eindeutig, dass die Entsprechung fi. hevonen 'Pferd' est. hobune id. - Fälle, die unregelmäßig sind und schon von z.B. ITKONEN und WIIK (s. o.) behandelt wurden - eindeutig Lehnwörter aus einer indogermanischen Sprache seien (er nennt als entsprechende Form das lautlich angeblich nahestehende griechische Wort für Pferd), da nicht nur die Entsprechung $e \sim o$ bei hintervokalischen Wörtern unregelmäßig sei, sondern auch $v \sim b$ (ebd. 91). Das finnische Wort ist jedoch hepo

\footnotetext{
${ }^{21}$ Den Laut $b$ klammert Holst (2001: 80) an dieser Stelle als eine jüngere Entwicklung aus $p$ aus. Er bezeichnet es als Laut, „dessen phonetische Interpretation umstritten“ sei.

${ }^{22}$ Dass die Entsprechung fi. sota est. sõda 'Krieg' im Estnischen eine Entlehnung aus einer anderen ostseefinnischen Sprache sei, ist eine unwahrscheinliche Theorie, für die eine entsprechende Begründung fehlt. Mehr s. Analyse der Kurzvokale im Estnischen (Kapitel 5.3).
} 
'Pferd' zuzuordnen (SSA I: 156) und somit liegt im Konsonantismus des Wortes keine Unregelmäßigkeit vor.

Trotz einiger Unstimmigkeiten in der Zusammenstellung seines Materials und einiger Ungenauigkeiten in der Analyse dessen lässt sich sagen, dass HOLST hier einen guten Ansatz verfolgt. Es ist wesentlich, dass er ein deutlich größeres Korpus betrachtet, als es bei vielen seiner Vorgänger der Fall gewesen ist. Anhand der Menge des Materials lassen sich deutliche Tendenzen der lautlichen Entwicklung im Estnischen feststellen. Diese hätte man bei einem kleineren Korpus nicht so deutlich erkennen können. In allen weiteren Bereichen liefert HoLST trotz des Umfangs an Daten, die er untersucht, von seinem Labialitätsgesetz abgesehen keine neuen Erkenntnisse und knüpft somit nur an das bisher bekannte Wissen an.

Auch KARL PAJUSALU (2012: 215) schließt sich der Theorie, dass es sich bei $\tilde{o}$ um eine Innovation der Südgruppe der ostseefinnischen Sprachen handelt, an. Neben der Frage, ob õ primär oder sekundär sei, stellt er die Aussage in den Raum, dass es zwei verschiedene Phoneme $\tilde{o}$ geben müsse (ebd.), nämlich $\underline{e}$ und $\underset{i}{ }$. Er führt an, dass im Südestnischen und im Kurlandlivischen zwei unterschiedliche ungerundete Hintervokale existieren sollen und die Unterschiede zwischen diesen beiden Vokalen sollen in der Höhe der Vokale bestehen: So sei im Südestnischen der mittelhohe Vokal $\tilde{o}$. Der hohe Vokal, auch hinteres $i$, werde in einigen Schreibungen durch $y$ markiert. Im Kurlandlivischen gebe es den hohen Vokal $\tilde{o}$ und den mittelhohen Vokal $\dot{o}$ (ebd.):

$\begin{array}{ccc} & \text { Südestnisch } & \text { Livisch } \\ \text { Mittelhoch } & \tilde{o} & \dot{o} \\ \text { Hoch } & \underline{i}(y) & \tilde{o}\end{array}$

Das Südestnische $y$ soll in verschiedenen phonetischen Umgebungen vorkommen, das mittelhohe $\dot{o}$ im Kurlandlivischen soll es seiner Meinung nach dagegen nur in betonten Silben nach labialen Konsonanten, die historisch $o$ beinhalteten, gegeben haben (ebd. 216). Trotz seiner Unterscheidung ist PAJUSALU (ebd.) der Meinung, dass der Laut $\dot{o}$ als spätes Allophon von /o/ gesehen werden könne und revidiert mit dieser Aussage seine eigene Theorie.

$\mathrm{Zu}$ bemerken ist, dass PAJUSALU (ebd.) davon ausgeht, dass $i \underset{i}{ }$ älter sei als $e$, sogar möglicherweise älter als die ostseefinnische Gemeinsprache. Damit widerspricht er eindeutig ITKONENS Positionen (s. o.), ohne auf sie Bezug zu nehmen. Auffällig ist daran nämlich, dass sich PAJUSALU mit der Frage nach dem Alter und der Klassifizierung von $\tilde{o}$ beschäftigt, ohne jedoch 
einschlägige Literatur wie den Artikel von ERKKI ITKONEN und dessen Analyse von E. N. SETÄLÄS Ansichten in irgendeiner Form zu rezipieren oder auch auf andere Arbeiten, die sich mit dieser Fragestellung befassen, einzugehen.

Erst später soll sich /õ/ im Nordestnischen und Wotischen verbreitet haben (ebd. 217). Die Beispiele, die PAJUSALU (ebd. 216) im Verlauf seiner Ausführungen nennt, versäumt er näher zu beschreiben. Sie lassen sich zwei verschiedenen Gruppen zuordnen: Entweder die Wörter bestehen in der ersten Silbe aus einer Verbindung eines dentalen Konsonanten (in seinen Beispielen $t$ oder $s$ ) mit einem (scheinbar beliebigen) Vokal, wobei letzterer vom Dental beeinflusst wird, sodass es hinterher eine Verbindung aus Dental und $i$ gibt, z.B. estS. sisar 'Schwester' estN. sõsar id. fi. sisar id., oder es handelt sich um Wörter mit Diphthongen in der ersten Silbe, bei denen das $i$ als zweiter Bestandteil auf den vorausgehenden Vokal einwirkt, z.B. estS. hỉim 'Stamm' estN. hõim id. fi. heimo id.

Zudem geht PAJUSALU (ebd. 217) davon aus, dass baltische und germanische Lehnwörter bei der Entwicklung von $\tilde{o}$ eine Rolle gespielt haben. Er ist nicht der erste, der diesen Zusammenhang erkennt, denn dies stellte vor ihm bereits HAKULINEN (s. o.) fest. PAJUSALus Beschreibungen sind jedoch etwas ausführlicher als die HAKULINENS. Er nimmt an, dass die besagten Lehnwörter an der Verbreitung des Lautes $\tilde{o}$ beteiligt gewesen sein könnten. In den nördlichen Sprachen der ostseefinnischen Sprachgruppe und im Südestnischen sei der Erstsilbenvokal $a$ der Lehnwörter erhalten geblieben, während es im Nordestnischen und Wotischen den Lautwandel $a>\tilde{o}$ gegeben habe. Als Beispiel nennt PAJUSALU estN. lõng 'Garn' wot. lõnka [sic!] id. estS. lang id. liv. lānga id. Ähnlich verhalte es sich ihm zufolge mit Stämmen, deren Erstsilbenvokal $o$ war, z.B. estN. oppida 'lernen' $\sim$ wot. oppõa id. $\sim$ estS. oppi id. $\sim$ liv. oppõ id. Dabei soll der Vokal $o$ in den meisten Sprachen erhalten geblieben sein, während er sich im Nordestnischen und Wotischen zu $\tilde{o}$ gewandelt hat. Die Herausbildung von $\tilde{o}$ soll zeitgleich mit der Übernahme der Lehnwörter mit $e$-Stämmen geschehen sein. Als Fazit nimmt PAJUSALU (ebd.) an, dass sich das mittelhohe $\tilde{o}$ im Südostseefinnischen sekundär neben $\underline{i}$ entwickelt haben müsse, bevor es zu einer Ausbreitung ins Nordestnische und Wotische gekommen sei.

Zwei Jahre später folgt zum Verhältnis der livischen Dialekte von PAJUSALU (2014) ein weiterer Artikel, The relationship between Salaca Livonian and Courland Livonian dialects, in dem er sich erneut mit dem $\tilde{o}$ der ersten Silbe auseinandersetzt. Dort definiert er das Auftreten des Lautes wie folgt (ebd. 154): 
In word-initial syllables bearing primary stress, all Livonian dialects, similarly to other southern Finnic languages, have featured the back unrounded vowel $\tilde{\boldsymbol{o}}$. This vowel, which has been referred to as a back $e$, has in fact been a high vowel in Livonian, i.e. $l$ [...]. Due to secondary labialization, $\tilde{o}$ in Salaca and western Courland Livonian has developed in the direction of $\ddot{u}$; in western Courland Livonian this vowel has later become $i$, for cases of both long and short $\tilde{o}$.

In seiner Betrachtung führt PAJUSALU (ebd. 154f.) seine Annahmen zur Entwicklung dieses Vokals im Livischen aus und gibt eine tabellarische Übersicht mit insgesamt 45 Beispielen (ebd. 156f.), die Auskunft darüber geben sollen, welche die salis-livischen und west-kurlandlivischen Entsprechungen von $\tilde{o}$ in der ersten Silbe im Ost-Kurlandlivischen sind. In dieser Tabelle rekonstruiert er außerdem die urlivischen Formen der Beispiele und das, was er für die vorlivische Zeit annimmt. Dabei kommt PAJUSALU (ebd. 155) zu der Schlussfolgerung, dass $\tilde{o}$ im Ost-Kurlandlivischen häufiger vorliege als im Salis- oder West-Kurlandlivischen. Er geht davon aus, dass das Urlivische bereits den Vokal $\tilde{o}$ kannte und sich von dort ausgehend erst die drei Dialekte entwickelt haben (ebd. 154). Gemeint ist mit diesem Laut der hohe Hintervokal $i$, für den PAJUSALU die Orthografie $l$, benutzt. Dieser sei im Ost-Kurlandlivischen als $\tilde{o}$ bewahrt, während er in den anderen beiden Dialekten labialisiert und zu $\ddot{u}$ geworden sei. Daraufhin habe sich $\ddot{u}$ im West-Kurlandlivischen zu $i$ weiterentwickelt. Der Lautwandel zu $\ddot{u}$ sei jedoch nicht gleichmäßig passiert, wie einige Beispiele belegen sollen, z.B. Sal. küza 'Zorn' Kur. kõzā, $k \bar{u} r d$ 'Zeit'. Die Erklärung dafür sei, dass das primäre $\ddot{u}$ im Salis-Livischen die Wandel $k>k$ und $s>\check{s}$ bewirkt haben soll, ehe es selbst zu $u$ wurde. Vor historischem $\tilde{o}$ habe der Lautwandel nicht stattgefunden. Im Salis-Livischen gebe es auch einige Entsprechungen von $u$, wie beispielsweise Sal. jua KurW. juva KurO. jõva 'gut' (ebd. 155).

Im weiteren Verlauf der Erklärungen gibt es noch einige Probleme mit PAJUSALUS Thesen, auf die aufgrund des Umfangs nur in Auszügen eingegangen werden soll. Bereits erwähnt wurde das sog. hintere $i$, das PAJUSALU (ebd. 158) als spätostseefinnischen Laut für die südlichen Sprachen annimmt. In drei rekonstruierten Vorstufen seiner Beispiele ist dieser Laut zu finden, nämlich in *sısar 'Schwester', *ssna 'Wort' und *siira 'Käse' (ebd. 156). Zu diesen Beispielen ist zu ergänzen, dass es sich bei sısar 'Schwester' fi. sisar id. est. sõsar id. sehr wahrscheinlich um ein Lehnwort aus dem Baltischen handelt, vgl. lett. sesuõ 'Schwester' (SSA III: 187). Bei KurO. sõnā 'Wort' mitsamt Entsprechungen ist von einem ostseefinnischen Wort auszugehen, für das jedoch bisher keine Etymologie vorliegt (ebd. 153f.). Problematisch ist das Verhältnis, in dem PAJUSALUS Rekonstruktion mit den Wörtern der ostseefinnischen Sprachen im Norden steht. Dort liegt in allen Sprachen die Form sana vor, die sich über eine Annahme von 
*sına nicht erklären lässt. Warum sollte also õ ursprünglich sein, wenn das restliche Sprachmaterial diese Vermutung nicht stützt? Bei stira 'Käse' handelt es sich um ein Lehnwort aus dem Slawischen (EES 493). Letzteres erkennt PAJUSALU (2014: 158) zwar, er berücksichtigt aber nicht, dass das wahrscheinlich russische Ausgangswort keinen Diphthong beinhaltet: russ. cblp 'Käse'. Auffällig ist auch der russische Laut $b l$ als solcher; es muss hier also von einem Einfluss des slawischen Vokalismus bei der Weitergabe dieses Lehnwortes ins Ostseefinnische ausgegangen werden, also $b l \sim \tilde{o}$. Eine weitere Möglichkeit bei der Übernahme wird durch BLOKLAND (2009: 269) aufgezeigt. Er vermutet nämlich, dass das livische Beispiel möglicherweise auch eine Entlehnung über das Estnische ${ }^{23}$ oder Lettische sein könnte.

Am Ende seiner tabellarischen Ausführung listet PAJUSALU (2014: 159) drei weitere Beispiele auf, die auf neuere lettische bzw. deutsche Lehnwörter zurückgehen und er ist der Meinung, dass $\tilde{o}$ in diesen Wörtern im Ost-Kurlandlivischen ähnlichen Bedingungen unterliege, wie im Urlivischen. Besonders problematisch ist jedoch, dass man das Alter der ausgewählten Lehnwörter berücksichtigen muss: Sie sind nämlich nicht, wie PAJUSALUS Tabelle den Eindruck erwecken will, bereits ins Urlivische eingetreten, sondern einzeln in die jeweiligen Dialekte (WINKLER 2011: 231), sodass aufgrund ihrer von Anfang an unterschiedlichen Entwicklung eine direkte Gegenüberstellung in der Analyse des Vokalismus nicht zielführend ist. ${ }^{24}$

Wie es schon zuvor andere für das Estnische getan haben, stellt auch PAJUSALU (2014: 158) fest, dass die Labialität der lautlichen Umgebung für das Livische eine Rolle zu spielen scheint: Ihm zufolge wurde osfi. $o$ im Urlivischen zu $\tilde{o}$, wenn der nachfolgende Konsonant labial war. Gleiches gelte für $\ddot{u}$, das im Salis- und West-Kurlandlivischen bewahrt sei, während im OstKurlandlivischen $\tilde{o}$ in hintervokalischer Umgebung vorliege (ebd.). PAJUSALUS Rekonstruktion des vorlivischen *hopeinen 'Pferd' ist problematisch, da sie augenscheinlich das Finnische und dessen Vokalismus nicht berücksichtigt, nämlich fi. hevonen bzw. hepo. Für die Diphthonge nimmt PAJUSALU (ebd. 159) ebenfalls den Einfluss der Labialität auf den ersten Bestandteil des Diphthongs an. Der Vokalismus war dabei anscheinend kein ausschlaggebender Faktor, denn die von ihm aufgeführten Beispiele beinhalten in der Folgesilbe Hintervokale oder neutrale Vokale.

\footnotetext{
${ }^{23}$ Auch EES (493) erwägt eine Entlehnung der livischen Entsprechung aus dem Estnischen. Diesem Wörterbuch zufolge ist der Entlehnungszeitpunkt nur schwer bestimmbar; es könne sich um eine altrussische Entsprechung handeln, aber genauso um ein jüngeres russisches Lehnwort.

${ }^{24}$ PAJUSALU (2014: 159) spricht lediglich von „German“, also deutschen Lehnwörtern, während sich WINKLER (2011: 231) auf mittelniederdeutsche Entlehnungen bezieht. Erwogen werden kann auch bei PAJUSALUS Überblick, dass er mittelniederdeutsche Lehnwörter meint, vgl. ALS sowie LW (28, 373).
} 
Schwierig ist auch der Fall, den PAJUSALU (ebd.) für den Diphthong $\tilde{o} i$ im Ost-Kurlandlivischen beschreibt. Anhand seiner rekonstruierten Formen wird deutlich, dass er eine Entwicklung von sposfi. *oi zu urliv. * $\tilde{o}$ annimmt. Aus diesem * $\tilde{o}$ sollen sich dann KurO. $\tilde{o} i$, Sal. $\bar{u}$ und KurW. $\bar{l}$ entwickelt haben. Unklar bleibt, warum * $\tilde{o}$ im Ost-Kurlandlivischen diphthongiert worden sein soll. Gleichzeitig beschreibt er in seinem nächsten Beispiel eine Entwicklung, in der sposfi. *oi $>$ urliv. * õi. Dies wiederum habe sich in KurO. $\tilde{o} i$, KurW. $\bar{\imath}$ und Sal. ei entwickelt. Wieso sich diese Entwicklung vom direkt vorausgehenden Beispiel unterscheidet, lässt PAJUSALU offen. Ferner bedient sich PAJUSALU (ebd. 157, 159) in zwei Beispielen der Formen aus dem livischen Wörterbuch von SJÖGREN-WIEDEMANN aus der Mitte des 19. Jahrhunderts und versucht diese zu erklären. Während er für das Spätostseefinnische noch einen Diphthong * eü ansetzt, sollen sich die Beispiele über urliv. * $\bar{o}$ zu õö entwickelt haben. Das Problem mit dem genannten Wörterbuch ist, dass die dortigen Einträge nicht einfach unkommentiert übernommen und mit den anderen livischen Entsprechungen gleichgesetzt werden können. ANDREAS JOHANN SJÖGRENS Aufzeichnungen müssen interpretiert werden, weil sie ungenau waren und weil er „,kulturpolitisch [bedingte] Eingriffe in die Sprache“" vorgenommen hat (WINKLER 2010: 372). Hinzu kommt, dass SJÖGREN die Arbeit an seinem Wörterbuch selbst nicht mehr abschließen konnte, da er bereits vor dessen Vollendung starb. Zwar wurde es durch FERDINAND JOHANN WIEDEMANN später noch fertig gestellt, allerdings erschwert dieser Umstand den Umgang mit dem salis-livischen Material nur zusätzlich (ebd. 372). Warum PAJUSALU überhaupt Formen aus dem Wörterbuch von SJÖGREN-WIEDEMANN benutzt und analysiert, anstelle sich auf das eindeutige livische Material zu stützen, motiviert er in seinen Ausführungen genauso wenig wie die Tatsache, dass er dies nur an den genannten beiden Stellen und nicht konsequent für jedes Beispiel macht.

Das Fazit, das PAJUSALU (2014: 159) am Ende seiner Untersuchungen hinsichtlich des livischen Vokalismus der ersten Silbe zieht, ist, dass das Ost-Kurlandlivische der urlivischen Sprachform entsprochen haben müsse, also dass dort ein altes $\tilde{o}$ vorliege. Die Formen im Salis- und WestKurlandlivischen sollen demnach Innovationen beinhalten. Die Frage, ob o alt sei und schon im Spätostseefinnischen existiert hat, beantwortet er in diesem Artikel nicht, obwohl er einige Beispiele mit einem solchen Laut ( $i$ bzw. $l$ ) für das Urlivische rekonstruiert (s. o.). Seine Ausführungen zu einem zweiten mittelhohen Hintervokal $\dot{o}$ sind nicht einschlägig und werden daher hier nicht gesondert diskutiert.

PAJUSALU scheint in der Analyse seiner beispielhaften Entsprechungen auch in diesem Artikel keine der relevanten Literatur zur livischen Lautgeschichte zu berücksichtigen, wenn es um die 
Frage nach õ geht (z.B. Posti (1942) oder auch KETTUNEN (1938), s. o.). Seine Thesen stützen sich ausschließlich auf die in der Tabelle vorliegenden Beispiele und seine anscheinend selbst rekonstruierten Urformen; er liefert zudem kaum Erklärungen oder Begründungen seiner Annahmen, sodass mitunter keine deutliche Motivation dafür zu erkennen ist, wie die Entwicklung der einzelnen Stufen abgelaufen sein soll. Inwieweit sich seine Rekonstruktionen des sog. „PreLivonian“" an das Ostseefinnische insgesamt anschließen lassen, bleibt ebenfalls unbeantwortet. Ferner wird für das Salis-Livische in seiner Analyse immer $\ddot{u}$ angenommen, außer der Laut folgt $k$ oder $\check{s}$. Dass es sich auch in anderen Positionen um ein $\tilde{o}$ mit der Schreibung < ü> handeln könnte, wird von Pajusalu nicht erwogen. Die Regel, die Pajusalu (2014: 154) zu Beginn seiner Analyse aufstellt und somit die Entsprechungen innerhalb des Livischen definiert, nämlich die Entsprechungen von Sal. $\ddot{u} \sim$ KurW. $\tilde{o} \sim$ KurO. $i$, stützt er nicht ausreichend, sodass nicht klar ist, ob man es hier tatsächlich mit einer historisch primären Vertretung zu tun hat oder ob der Lautstand nicht in Wirklichkeit ein anderer war.

\subsection{Zusammenfassender Rückblick}

Die vorausgegangene Darstellung des bisherigen Forschungsstandes hat deutlich die Komplexität und die Streitbarkeit des Themas gezeigt, genauso aber auch, wie wichtig es ist, der Frage nach der Herkunft und Entstehung von $\tilde{o}$ weiterhin nachzugehen. Die Diskussion um das tatsächliche Alter von $\tilde{o}$ lässt sich nur mit einer fundierten und ausführlichen Analyse, die alle bisherigen Positionen zusammenbringt und mit Fakten be- oder widerlegt, beilegen. Dabei gilt es auch, mehr als nur eine Einzelsprache für sich zu untersuchen und dadurch einen Blick auf das große Ganze zu werfen. Da dies in der Forschung bisher nicht geschehen ist, bleibt das Thema weiterhin aktuell.

Einige Aspekte sind durch die Diskussion des aktuellen Forschungsstandes besonders deutlich geworden: Auffällig in der Betrachtung der Wissenschaftsgeschichte ist vor allen Dingen eine bislang eher spärlich vorliegende Analyse des südestnischen, livischen und wotischen Sprachmaterials. Wie die Darstellung gezeigt hat, wurde sich bei den Untersuchungen hauptsächlich auf den Vergleich zwischen dem Finnischen und dem Estnischen gestützt. Das ist insofern naheliegend, da das Material zunächst weit umfassender und zugänglicher war. Für das Livische gab es lange Zeit nur das Wörterbuch von KeTtUNEN (1938; LW) als umfassende Quelle. ${ }^{25}$

\footnotetext{
${ }^{25}$ Zum Wörterbuch von A. J. SJÖGREN (1861) s. o.
} 
Nicht zuletzt durch die Herausgabe eines livischen Wörterbuchs mit der an die neue Standardisierung angepassten Orthografie (LELS = Lìvõkiel-ēstikiel-leţkīel sõnārōntõz) im Jahr 2012 und die Publikation der komplettierten und überarbeiteten Gesamtausgabe des wotischen Wörterbuchs $^{26}$ im Jahr 2013 (VKS = Vadja keele sõnaraamat) sowie dem Erscheinen der Wörterbücher EVS und VES hat sich dieser Umstand für die Sprachen maßgeblich geändert.

Dass die recht einseitige Betrachtungen und der direkte Vergleich von nur einer Sprache mit $\tilde{o}$ mit dem Finnischen (mit Ausnahme von VIITSO) bisher nicht ausreichend waren, ist aus der Diskussion der Wissenschaftsgeschichte deutlich hervorgegangen. Daher müssen die Untersuchung und die Gegenüberstellung der Sprachen unbedingt um das Wotische, Livische und Südestnische erweitert werden, um überhaupt eine Aussage über den Vokalismus des Ostseefinnischen insgesamt machen zu können. Wie soll sonst geklärt werden, wie sich der Laut $\tilde{o}$ der ersten Silbe entwickelt hat, wenn nicht alle Sprachen, in denen er vorkommt, in die Untersuchung mit einbezogen werden? Die lautlichen Verhältnisse im Vokalismus des Samischen, Mordwinischen und Marischen hat ITKONEN wie oben gezeigt bereits ausreichend behandelt, sodass in weiterführenden Kontexten auf seine Theorie zurückgegriffen werden kann. ITKONENS Erkenntnisse gehen auch mit den Annahmen SAMMALLAHTIS über den wahrscheinlichen Lautstand der finnougrischen und uralischen Grundsprache einher (s. o.). Da anscheinend keine Fortsetzer von $\tilde{o}$ aus dem Uralischen bis in die ostseefinnischen Sprachen hinein existieren, ist SAMmaLlaHTIS Hypothese als Referenz für die besagte Sprachstufe im Rahmen dieser Analyse als ausreichend anzusehen.

Ein weiterer Aspekt, der durch die Diskussion der Wissenschaftsgeschichte im Hinblick auf $\tilde{o}$ deutlich geworden ist, ist der Umstand, dass die Frage nach den Entsprechungen $o \sim \tilde{o}$ bislang nicht abschließend geklärt werden konnte. Dies liegt mitunter daran, dass sich die vorhandenen Arbeiten entweder gar nicht mit diesem Problem beschäftigt, es als eine Art Nebenprodukt in der Lautentwicklung abgehandelt oder nur Hypothesen in den Raum geworfen haben. Die durch ihre Regelmäßigkeit leichter erklärbaren Entsprechungen $e \sim \tilde{o}$ hat man bereits von Beginn an weit ausführlicher, wenn nicht sogar zu Genüge behandelt und man konnte sogar die Bedingungen für diesen Lautwandel identifizieren. Lediglich JAN HENRIK HOLST (s. o.) versucht

${ }^{26}$ Der erste Band der mehrbändigen Ausgabe des wotischen Wörterbuchs erschien bereits im Jahr 1990 (VKS: 20). 
2001 - also über 100 Jahre nachdem die Problematik innerhalb der Erforschung der ostseefinnischen Lautgeschichte zum ersten Mal wissenschaftlich behandelt wurde - mit seinem Labialitätsgesetz eine deutlichere Erklärung für einen wesentlichen Teil der abweichenden Entsprechungen $o \sim \tilde{o}$ im Estnischen zu liefern. Nichtsdestotrotz bleiben auch nach seiner umfassenden Analyse noch sehr viele Fragen offen, nicht zuletzt, weil er ausschließlich das Estnische und das Finnische miteinander vergleicht und somit keine Daten dazu vorliegen, ob sein Labialitätsgesetz für das Wotische und das Livische überhaupt zutreffend ist oder ob die Entwicklung hier, aber auch im Estnischen womöglich sogar ganz anders abgelaufen sein könnte. Dass Letzteres für das Livische zu vermuten ist, hat bereits ITKONEN (s. o.) angedeutet, als er den Lautwandel $o>\tilde{o}$ in seinem Artikel für unregelmäßig erklärt hat.

Abschließend lässt sich sagen, dass also insbesondere bei den Entsprechungen $o \sim \tilde{o}$ weiterhin starker Forschungsbedarf besteht. Auch in diesem Material zeigt sich, dass sich der Lautwandel bei über der Hälfte der infrage kommenden Entsprechungen nicht über HoLSTS Labialitätsgesetz erklären lässt. Es gilt herauszufinden, welche Bedingungen für diesen Lautwandel eine wesentliche Rolle spielen und was der Grund dafür ist, dass das ursprüngliche $e$ zwar einer offensichtlichen und eindeutigen Regelmäßigkeit unterliegt, der Lautwandel mit $o$ jedoch so stark schwankt und bisher nicht erklärt werden konnte, obwohl genug sprachliches Forschungsmaterial vorliegt. 


\section{Zum Vokalinventar der ostseefinnischen Sprachen}

Das folgende Kapitel befasst sich mit dem Vokalismus der ostseefinnischen Grundsprache und dem der untersuchten Einzelsprachen. Es gibt einen Überblick über die dort auftretenden Phoneme und deren Klassifizierung. Auf eine Darstellung des Konsonantismus wird in diesem Rahmen verzichtet. Er ist in den einschlägigen Arbeiten zu diesen Sprachen nachzuvollziehen. Für den estnischen Konsonantismus vgl. VIITSO (2003: 22), für den wotischen vgl. ARISTE (1968: 7) und für den livischen vgl. LELS (388). Einen Überblick über die Entwicklungen des südestnischen Konsonantismus bietet KEEM (2002: 23-33). Zum Konsonantismus der Grundsprache äußert sich ebenfalls VIITSO (1998a: 109f.).

Weitere lautliche Besonderheiten, wie beispielsweise die Betonung oder die Stufenwechselverhältnisse, werden an dieser Stelle nicht näher betrachtet, da sie für den Rahmen dieser Untersuchung keine größere Relevanz haben. Sie werden in den entsprechenden Lautlehren und Grammatiken der Einzelsprachen sowie sprachübergreifenden Arbeiten erläutert. ${ }^{27}$

\subsection{Der Vokalismus der ostseefinnischen Grundsprache}

Wie auch schon durch die Diskussion der Wissenschaftsgeschichte deutlich geworden ist, herrscht über den Lautstand der ostseefinnischen Grundsprache Uneinigkeit, je nachdem welche Laute für den ursprünglichen Vokalismus und Konsonantismus angesetzt werden. Da im Rahmen dieser Arbeit davon ausgegangen wird, dass der Laut o eine sekundäre Entwicklung ist, ist er für diese Sprachstufe folglich nicht anzusetzen.

VIITSO (1998a: 106) zufolge können acht Vokale als Bestandteil des ostseefinnischen Vokalismus rekonstruiert werden, wobei diese Monophthonge sowohl in kurzer als auch in langer Vertretung vorliegen konnten:

$\begin{array}{llll}*_{i} & *_{\ddot{u}} & & *_{u} \\ *_{e} & *_{\ddot{o}} & & *_{o} \\ *_{\ddot{a}} & & *_{a} & \end{array}$

Alle angeführten Vokale mit Ausnahme von $* \ddot{o}$ konnten in kurzer Form auch in der zweiten Silbe vorkommen und seien ein Zeichen für eine bereits teilweise vorhandene Vokalharmonie, da das Auftreten der einzelnen Laute in der zweiten Silbe an Bedingungen geknüpft gewesen

${ }^{27}$ Vgl. dazu u.a. LAANEST (1982: 93ff., 106-113). 
sei (ebd. 106f.): Die Vokale ${ }^{*} e, i$ und $*_{o}$ konnten in allen nichtersten Silben auftreten, während für die anderen Vokale Einschränkungen gegolten haben. Die Vokale $* \ddot{u}$ und $* \ddot{a}$ seien in der nichtersten Silbe nur aufgetreten, sofern vor ihnen $* e, * i, * \ddot{a}, * \ddot{o}$ oder $* \ddot{u}$ vorlagen. Für das Vorkommen von $* u$ und $* a$ in nichtersten Silben durften VIITSO (ebd. 107) zufolge die Laute $* \ddot{a},{ }^{*} \ddot{o}$ und $* \ddot{u}$ nicht vorausgehen. Der Vokal $\ddot{o}$ sei eine späte Entwicklung, da er wohl zunächst als Variante des Hintervokals $o$ in Onomatopoetika aufkam (LAANEST 1982: 115). ${ }^{28}$ In der zweiten Silbe sei er erst nach Auflösung der ostseefinnischen Grundsprache aufgekommen (ebd.), vgl. zur Vokalharmonie Kapitel 3.6.

Von den Langvokalen gelten $\bar{e}, \bar{l}, \bar{o}$ und $\bar{u}$ als alt und werden gelegentlich bereits für die finnougrische Grundsprache angesetzt. In einsilbigen Wörtern jedoch seien sie erst im Ostseefinnischen entstanden, als $n, v$ und $j$ geschwunden sind (LAANEST 1982: 114). Mit dem Schwund dieser Laute hänge auch die Entstehung der Diphthonge mit der zweiten Komponente $i, u$ oder $\ddot{u}$ zusammen (ebd. 114f.).

\subsection{Der estnische Vokalismus}

Der Vokalismus der estnischen Hochsprache kennt heute insgesamt neun Laute, die als Vorderund Hintervokale unterschiedlicher Höhe realisiert werden. Sie werden in der nachfolgenden Tabelle nach VIITSO (2003: 20) mit Ergänzungen nach ASU \& TERAS (2009: 368) dargestellt:

\begin{tabular}{ccccc} 
& \multicolumn{2}{c}{ Vordervokale } & \multicolumn{2}{c}{ Hintervokale } \\
& Illabial & Labial & Illabial & Labial \\
Hoch oder geschlossen & $i[\mathrm{i}]$ & $\ddot{u}[\ddot{\mathrm{u}}]$ & & $u[\mathrm{u}]$ \\
Mittelhoch & $e[\mathrm{e}]$ & $\ddot{o}[\varnothing]$ & $\tilde{o}[\gamma]$ & $o[\mathrm{o}]$ \\
Tief oder offen & $\ddot{a}[\mathfrak{x}]$ & & $a[\mathrm{a}]$ &
\end{tabular}

Das $\tilde{o}$ wird von ViITSO (2003: 20) als geschlossener als die anderen mittelhohen Vokale beschrieben. Je nach Sprecher kann die Aussprache dieses Lautes variieren. So reiche das Spektrum ASU \& TERAS (2009: 369) zufolge von einem mittelhohen Hintervokal [ $\gamma]$ über einen geschlossenen Hintervokal [u] bis hin zu einem mittelhohen Mittelvokal [९].

\footnotetext{
${ }^{28}$ In LÄGLOS (I: XIII) wird $\ddot{o}$ erst für das Späturfinnische angesetzt. Im Frühurfinnischen habe er noch nicht vorgelegen.
} 
Beim Vorkommen der einzelnen Vokale gibt es zudem einige Einschränkungen: In der ersten Silbe können sämtliche Qualitäten auftreten, während in der zweiten Silbe lediglich die Laute $a, e, i$, und $u$ vorkommen (VIITSO 2003: 21). Alle genannten Vokale gibt es auch noch in einer langen Vertretung, die jedoch nur in der ersten Silbe auftritt (ebd.). ${ }^{29}$

Das Estnische hat ferner eine Vielzahl an Diphthongen. Alle neun der angeführten Kurzvokale können als erster Bestandteil eines Diphthongs dienen, während an zweiter Stelle lediglich $a$, $e, i, o$ und $u$ auftreten können (ebd.). So ergeben sich als Diphthonge, deren erster Bestandteil $\tilde{o}$ ist, in der ersten Silbe die folgenden (ebd. 22): $\tilde{o}$, $\tilde{o} e, \tilde{o} a, \tilde{o u} .^{30}$

\subsection{Der wotische Vokalismus}

Das Wotische hat insgesamt zehn Vokalphoneme, die sich nach ARISTE (1968: 1) wie folgt darstellen lassen:

$\begin{array}{ccccc} & \begin{array}{c}\text { Labiale Vorder- } \\ \text { vokale }\end{array} & \begin{array}{c}\text { Vorder- } \\ \text { vokale }\end{array} & \text { Mittelvokale } & \text { Hintervokale } \\ \text { Hoch } & \ddot{u} & i & i & u \\ \text { Mittelhoch } & \ddot{o} & e & e & o \\ \text { Tief } & & \ddot{a} & & a\end{array}$

Die Artikulation der Vorder- und Hintervokale ähnele der estnischen (ebd.), genaue Messdaten zur Aussprache liegen zum Wotischen nicht vor. ARISTE (ebd.) beschreibt außerdem, dass $\underline{\varrho}$ dem estnischen $\tilde{o}$ entspreche, während $i$ ähnlich wie das russische $b l$ artikuliert werde und eine Entlehnung sei, die ausschließlich in russischen Lehnwörtern vorkomme. Nach neuer Orthografie schreiben sich die beiden Laute $\tilde{o}(=e)$ und $y(=i)(\mathrm{VKS} 33)$.

Alle Vokalphoneme gibt es in einer kurzen und einer langen Vertretung (ARISTE 1968: 2). Andere Quellen gehen davon aus, dass $\underline{i}$ im Wotischen als einziger Vokal keine lange Vertretung habe (NiKOLAEVA 1997: 268).

\footnotetext{
${ }^{29}$ VIITSO (2003: 21) weist an der Stelle darauf hin, dass $\ddot{u} \ddot{i}$ in der Ausprache zu einem Diphthong [üi] wird, wenn ihm direkt ein Vokal $a, e$ oder $u$ folgt, vgl. z.B. est. sü̈̈a 'essen' oder est. sü̈̈̈es 'während des Essens'.

${ }^{30}$ Ferner werden von VIITSO (2003: 22) an dieser Stelle auch Diphthonge angeführt, die lediglich in Entlehnungen auftreten. Dazu zählt eu. Zu den Diphthongen, die in der zweiten Silbe auftreten können, vgl. VIITSO (2003: 21f.).
} 
Das Wotische ist zudem reich an Diphthongen: ARISTE (ebd. 3f.) listet insgesamt eine Anzahl von 24 Diphthongen für die hauptbetonte Silbe auf, außerdem ebenso viele für die nebenbetonten Silben. Als Diphthonge mit $\tilde{o}$ als erstem Bestandteil werden für die erste und nichterste Silbe gleichermaßen $e a^{31}$, e $i$ und $e u$ genannt.

\subsection{Der livische Vokalismus}

Im livischen Vokalismus findet sich ein leicht umfassenderes Phoneminventar als in den beiden zuvor behandelten Sprachen. Von VIITSO \& ERNŠTREITS wird es in ihrem Wörterbuch wie in der folgenden Tabelle beschrieben (LELS 385). Dabei werden einerseits die kurzen, andererseits auch die dazu gehörenden langen Vertretungen der einzelnen Vokale aufgelistet ${ }^{32}$ :

$$
\text { Vordervokale }
$$

$\begin{array}{cccc}\text { Hoch } & i \bar{\imath} & \tilde{o} \overline{\tilde{o}} & u \bar{u} \\ \text { Mittelhoch } & e \bar{e} & \dot{o} \bar{o} & o \bar{o} \\ \text { Tief } & \ddot{a} \bar{a} & a \bar{a} & \bar{Q}\end{array}$

Auffällig ist, dass zwei Laute auftreten, die in den anderen untersuchten Sprachen unbekannt sind: $\dot{o}$ und $\bar{q}$. Bei $\dot{o}$ handelt es sich in PostIS (1942: XVII, 10) Transkription um $g$, das von ihm als „ein dem $o$ entsprechender Mittelzungenvokal mit reduzierter Lippenartikulation“ beschrieben wird und nur im Ost-Livischen nach $p, m$ und $v$ vorkomme. Das $\bar{Q}$ wird von ihm als $Q$ transkribiert und komme ebenfalls nur im Ost-Livischen vor. Es sei „ein $O$ mit etwas niedrigerer Zungenstellung" und trete immer nur in langer Vertretung auf (ebd.). ${ }^{33}$

Ferner kennt das Livische eine Vielzahl an Diphthongen, von denen jedoch nur ein gewisser Anteil für die vorliegende Betrachtung einschlägig ist, sowie einige Triphthonge. Zu nennen sind von den Diphthongen an dieser Stelle $\tilde{o} i$ und $\tilde{o u}$ (LELS 386f.; zu den Vertretungen mit Stoßton und den langen Entsprechungen vgl. ebd.); Triphthonge mit $\tilde{o}$ als Bestandteil gibt es nicht (ebd. 387).

\footnotetext{
${ }^{31}$ Bereits die von ARISTE (1968: 3) genannte Form seaza 'Krieg-INESS.' deutet darauf hin, dass es sich hierbei nicht um einen eigentlichen Diphthong handelt. Sie ist vielmehr das Ergebnis des Ausfalls von intervokalischem $t$ (VKÄ 61), vgl. (55) wot. sõta, gen. sõaa 'Krieg'.

${ }^{32}$ Messdaten zur Aussprache liegen auch zum Livischen nicht vor.

${ }^{33}$ Das $\tilde{o}$ liegt in der ersten Silbe nur im Ost-Livischen und im Dialekt von İra vor, im West-Livischen jedoch nicht (VIITSO 2011: 205f.).
} 
Für die unbetonten Silben werden die folgenden Laute angesetzt: In allen Dialekten können $i$, $a$ und $\tilde{o}$ sowie die langen Vertretungen $\bar{l}, \bar{e}, \bar{a}$ und $\bar{u}$ auftreten, im Dialekt Îra und im WestLivischen zudem der Monophthong $u$ (VIITSO 2011: 206). Hier sei an das eingangs beschriebene Orthografieproblem erinnert: in unbetonten Silben entspricht $\tilde{o}$ nicht dem Phonem der ersten Silbe, sondern einem reduzierten $\hat{\partial}$ (LW XVIII).

\subsection{Der südestnische Vokalismus}

Für die südestnischen Dialekte ${ }^{34}$ Mulgi und Tartu werden für die hauptbetonten Silben die gleichen Vokale angesetzt wie für das Standardestnische (TERAS 2003: 16). Im Dialekt von Võru komme noch ein Phonem $i$ hinzu, bei dem diskutiert wird, ob es sich tatsächlich um ein eigenständiges Phonem handelt, da keine Minimalpaare zu $e$ vorliegen (ebd.). Die Diskussion um $i$ wurde bereits im wissenschaftsgeschichtlichen Teil dieser Arbeit angedeutet (vgl. Kapitel 2.2) und soll in diesem Rahmen nicht weiter fortgeführt werden. $\mathrm{Zu}$ den Bedingungen des Auftretens von $\underline{i}$ vgl. auch KEEM (2002: 33, 36). PARVE (2000: 9) zählt außerdem die Allophone $i ̣$ und ę zu den Monophthongen hinzu. Ihr zufolge sieht eine schematische Darstellung des südestnischen Vokalismus wie folgt aus (ebd.):

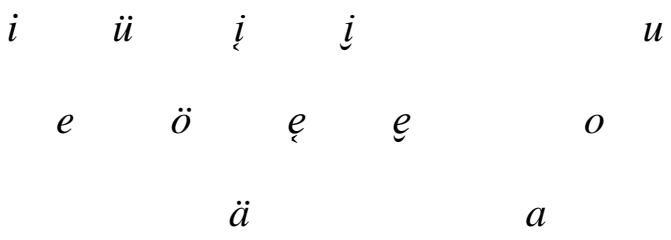

Laut PARVE (ebd. 14) werden $\underline{i}$ und $\varrho$ weiter vorne artikuliert als andere Hintervokale und unterscheiden sich in ihrer Artikulationshöhe von est. o. Die Artikulation von $e$ sei tiefer, die von $i$ höher.

\footnotetext{
${ }^{34}$ Das Südestnische wird als archaisch bezeichnet und man geht davon aus, dass die Nord- und Süddialekte (bzw. -sprachen) des Estnischen sich wohl unabhängig voneinander von der ostseefinnischen Grundsprache getrennt haben. Später haben sie sich wieder aufeinander zu entwickelt, was der geographischen Nähe geschuldet sei (WINKLER 2002a: 634). Bereits WIEDEMANN (1864: 1) erwähnt in seinem Versuch ueber den werroehstnischen Dialekt, dass das Estnische, welches im Süden des Landes gesprochen werde, dem Finnischen und Livischen in vielen Punkten näherstehe, gleichzeitig aber auch in einen fließenden Übergang zum „Revalehstnischen“, also dem Nordestnischen, gehe. Die Unterschiede zwischen dem Nord- und Südestnischen äußern sich nicht nur im lautlichen Bereich, sondern u.a. auch in Lexik und Syntax (WINKLER 2002a: 635).

Zum Südestnischen werden heute verschiedene Dialekte gezählt, die v.a. im Süden bzw. Südosten Estlands gesprochen werden: Mulgi, Tartu und Võru (EMS I: 10ff.). Das Dialektgebiet der Setu, das sich sowohl auf estnischem als auch auf russischem Staatsgebiet befindet, wird mitunter gesondert hinzugezählt (KEEM 2002: 5). Die Dialekte selbst werden ferner laut KEEM (ebd.) in vier große Dialektgebiete unterteilt, die Nord-, Süd-, Ost- und Westgruppe. Jedes dieser Gebiete unterteile sich schließlich in weitere Unterdialekte, deren einzelne Ausprägungen hier jedoch nicht näher diskutiert werden.
} 
Die Vokale können ebenso in einer langen Vertretung auftreten, zur Qualität vgl. auch TERAS (2003: 17f.). Alle Vokalphoneme treten ferner als erster Bestandteil von Diphthongen auf, während als zweiter Bestandteil lediglich $a, e, i, o, u, \ddot{a}, \ddot{o}$ und $\ddot{u}$ vorkommen (ebd.17). ${ }^{35}$ Diphthonge, in denen im Dialekt Võru $i$ auftrete, stehe in den anderen südestnischen Dialekten $e$ (ebd.).

Von Teras (2003: 18) werden für die Folgesilben die Vokale $i, i, u ̈, u, e, e, \ddot{o}, o, \ddot{a}$ und $a$ angesetzt, wobei $i \underset{\text { und }}{e}$ allophonisch seien und $o$ lediglich in der Nord- und Ostgruppe des Dialekts Võru vorkomme. Sie beschreibt $e$ als Laut, der die hintervokalische Variante zu $e$ sei und in seiner Qualität dabei eher dem $\varrho$ der ersten Silbe ähnele (ebd. 19).

\section{6}

\section{Zur Vokalharmonie}

Beschäftigt man sich mit dem Vokalismus der ostseefinnischen Sprachen, so stellt sich auch die Frage nach der Vokalharmonie und ihrer Relevanz für die Entwicklung des Lautes $\tilde{o}$. Unter Vokalharmonie versteht man, dass (nicht zusammengesetzte) Wörter in ihrem Vokalismus in allen Silben entweder nur vorder- oder nur hintervokalisch sein dürfen. Die Laute $e$ und $i$ sind im Falle der ostseefinnischen Sprachen üblicherweise neutral; sie können also sowohl mit Vorder- als auch Hintervokalen gleichermaßen kombiniert werden. Die Vokalharmonie ist ein Phänomen, das in vielen der finnougrischen Sprachen auftritt, ${ }^{36}$ und bereits für die ostseefinnische Grundsprache anzunehmen ist (vgl. LAANEST 1982: 129; ITKONEN 1945: 159).

Auch VIITSO (1998a: 106f.) setzt an, dass es eine teilweise Vokalharmonie bereits in der ostseefinnischen Grundsprache gegeben habe (vgl. auch Kapitel 3.1), da $\ddot{o}$ in nichtersten Silben anfangs nicht vorkam. Für $\ddot{o}$ gilt, dass es wohl später entstanden sei. LAANEST (1982: 115) beschreibt, dass es ,zunächst in onomatopoetischen Wörtern als Variante des Phonems $o$ auftrat"“. Erst durch das Entstehen einer Bedeutungsunterscheidung von $o$ und $\ddot{o}$ habe sich $\ddot{o} \mathrm{zu}$ einem eigenständigen Laut entwickeln können. Ein $\ddot{o}$ in nichtersten Silben wird von LAANEST (ebd.) erst für die Zeit nach der gemeinsamen ostseefinnischen Grundsprache angesetzt. Als Beweis dafür nennt er u.a. das Wotische, das eine Vokalharmonie bei $\ddot{a}$ und $\ddot{u}$ kenne, nicht aber bei $\ddot{o}$ : in nichtersten Silben stehe dann $o$, z.B. fi. näkö 'Gesicht, Sehvermögen' wot. näko 'Gesicht (Antlitz)'. VIITSO (1998a: 107) nimmt ebenfalls an, dass es sich bei der Entstehung von

\footnotetext{
${ }^{35}$ Für eine tabellarische Übersicht s. TERAS (2003: 17f.).

${ }^{36}$ Zur Vokalharmonie in den uralischen Sprachen vgl. COMRIE (1988: 454-457).
} 
$\ddot{o}$ in nichtersten Silben um eine Entwicklung $*_{o}>*_{o}: *_{0}$ handele. Diese habe zuerst in den Norddialekten der ostseefinnischen Sprachen stattgefunden und zu einem Ausbau der Vokalharmonie geführt. ${ }^{37}$

Die Vokalharmonie liegt heute nicht mehr in allen ostseefinnischen Sprachen vor. Während sie z.B. im Finnischen, Wotischen ${ }^{38}$ und Südestnischen ${ }^{39}$ bewahrt wurde, hat es im (Nord-)Estnischen und Livischen einen deutlich erkennbaren Abbau dieses Systems gegeben. Die Laute $\ddot{a}$, $\ddot{o}$ und $\ddot{u}$ kommen in den letztgenannten Sprachen in der zweiten Silbe nicht (mehr) vor (LAANEST 1982: 129; vgl. auch VIITSO 2003: 20). Im Wotischen ist eine besonders interessante Erscheinung die Tatsache, dass $e$ keinesfalls neutral ist, sondern im Kontext der Vokalharmonie der vordervokalische Gegenpart zu o (WINKLER 2002b: 762; LAUERMA 1993: 54f.). Eine Vokalharmonie in Bezug auf o kennt auch das Südestnische (KEEM 1997: 7): es trete in nichterster Silbe auf, wenn in vorausgehenden Silben $a, o$ oder $u$ stehen.

Als Beispiel für die bewahrte Vokalharmonie sei fi. kylmä 'kalt; Kälte, Frost' wot. tšülmä id. $\sim$ estS. külm, gen. - $\ddot{a}$ id. genannt, für die abgebaute dagegen est. külm, gen. $-a$ id. $\sim$ liv. kīlma, külma id.

Zur Annahme einer ursprünglichen Vokalharmonie im Kontext von $\tilde{o}$ kommt bereits ITKONEN (1945: 159) in seinem Artikel über die Mittelvokale im Ostseefinnischen (vgl. Kapitel 2.2). Auch setzt er die Neutralität der Laute $e$ und $i$ voraus, die zudem in velarer Umgebung leicht velarisiert gewesen sein können. Diese Theorie ITKONENS ist auch Voraussetzung für die nachfolgende Analyse.

\footnotetext{
${ }^{37}$ Wie bereits im wissenschaftsgeschichtlichen Teil beschrieben, geht ITKONEN (1945: 174f.) davon aus, dass die Voraussetzung zur Herausbildung von $\ddot{o}$ die Neutralität von $e$ und $i$ gewesen sei.

${ }^{38}$ Zur wotischen Vokalharmonie und deren Abweichungen vgl. auch ARISTE (1968: 4f.).

${ }^{39} \mathrm{Zu}$ den Bedingungen vgl. KeEM (1997: 6f.)
} 


\subsection{Zum Aufbau des Korpus}

Das Korpus teilt sich anhand der übergeordneten Kategorien in vier Unterkapitel: Kurzvokale, Langvokale, Diphthonge und Sonderfälle. Innerhalb dieser Kapitel sind die Belege alphabetisch sortiert und werden in bereits bekannter Reihenfolge angeführt (Estnisch - Wotisch - Livisch - Südestnisch, sofern $\tilde{o}$ in allen Sprachen in der ersten Silbe auftritt. Anderenfalls wird mit der ersten einschlägigen Entsprechung begonnen). Falls vorliegend, werden eine finnische Entsprechung sowie eine rekonstruierte Urform einer früheren ostseefinnischen Sprachstufe angeführt. Daran anknüpfend folgen in relevanten Fällen Angaben zur Etymologie des jeweiligen Belegs und schließlich wesentliche Informationen zur lautlichen Entwicklung in den Einzelsprachen. Für die Zuordnung in der späteren Analyse werden alle Belege zudem fortlaufend nummeriert.

\subsection{Einschlägige Lautwandel}

Im Vorfeld zur Analyse des eigentlichen Korpus soll zunächst auf Lautwandel eingegangen werden, die in den untersuchten Sprachen relevant sind. Dabei wird sich v.a. auf vier wesentliche Quellen bezogen: Eestin kielen äännehistoria (EKÄ; Die Lautgeschichte der estnischen Sprache, 1962), Vatjan kielen äännehistoria (VKÄ; Die Lautgeschichte der wotischen Sprache. 1930), beide von KetTunen, und für das Livische Postis Grundzüge der livischen Lautgeschichte $(1942)^{40}$. Für das Südestnische finden ebenso v.a. EKÄ sowie KeEMs Võru murde tekstid (Texte des Dialekts Võru, 2002) Verwendung. Die Darstellung der Lautwandel erfolgt in bewährter Reihenfolge. Bei der späteren Beschreibung des Korpus werden die Entwicklungen mit ihrem jeweiligen Kürzel angeführt.

\subsubsection{Lautwandel im Estnischen}

e:1 $\quad *_{-} \ddot{a}->-a-$ in nichterster Silbe (EKÄ 146f.)

est. härjad 'Ochse-PL' fi. härät id.; est. pesa 'Nest' fi. pesä id.

\footnotetext{
${ }^{40}$ Hinzuweisen ist hier auf Postis (1942: XVIIf.) Notation der nach ihm zitierten Belege, in welcher u.a. der Stoßton eigentümlich markiert wird, vgl. liv. kăggâl 'Hals' vs. ka 'ggâl id. (LW 100).
} 
e:2 $*_{-} o_{-}>-u$ - weiter hinten als in der ersten Wortsilbe (EK ̈̈ 155)

est. janu 'Durst' fi. jano id.; est. palju 'viel' fi. paljon id.

e:3 Schwund des vokalischen Auslauts - in zweisilbigen Wörtern bei langer erster ${ }^{41}$ und offener zweiter Silbe, in mehrsilbigen Wörtern immer (EKÄ 168) est. jalg 'Bein, Fuß' fi. jalka id.; est. laul 'Lied' fi. laulu id.

e:4 Schwund des Dentals nach unbetonter Silbe (EKÄ 78; LAANEST 1982: 205) ${ }^{42}$ est. hõbe 'Silber' < fosfi. * šope- $\delta a$

e:5 Vokalisierung des Klusils * $k$ im Silbenauslaut vor - $l$ - (EK ̈̈ 86) est. eile 'gestern' fi. eilen id. < *eklen; vgl. auch est. nõel 'Nadel'

e:6 *-uhh- > (-hu-) $-h v-($ EK ̈̈ 98)

est. jõ hv, gen. -e, -i 'Pferdehaar, Rosshaar' fi. jouhi 'Rosshaar'

e:7 $\quad *_{-} l h->-l-($ EKÄ 100)

est. sulane 'Knecht', vgl. fi. sulhanen 'Verlobter, Bräutigam'

e:8 Vokalisierung von $* n$ in Verbindung * $n s: *_{-} n s^{-}>-V s^{-}($EK ̈̈ 104)

est. põõsas 'Busch' fi. pensas id.; est. maasikas 'Erdbeere' fi. mansikka id.

e:9 Ausfall von *-m- (EKÄ 108)

est. põrand 'Fußboden' fi. permanto 'Fußboden; Dielenbrett'

$\mathrm{e}: 10 \quad-v-\sim-u-($ EK ̈̈ 115)

est. põu, gen. - $e$ 'Brust' fi. povi 'Busen, Brust'

e:11 Schwund von $*_{-v}$ - vor oder nach Labialvokal (EK ̈̈ 116)

est. nõu 'Rat, Ratschlag' fi. neuvo 'Rat; Art und Weise; Ratschlagung [...]'

e:12 $-u$ - ist eine reflexive Ableitung, $<* \ddot{u}$ (LEHTISALO 1936: 40)

est. hukkuma 'verderben, zugrunde gehen'

e:13 le- ist Ableitungssuffix (KASIK 2015: 160ff.)

est. tegelema 'sich beschäftigen, sich befassen', vgl. est. tegema 'etw. tun'

e:14 Ableitungssuffix -ke(ne) (KASIK 2015: 251)

est. aknake 'Fensterchen'

\footnotetext{
${ }^{41}$ Zur Länge der Silben vgl. auch VISK $\$ 11$.

${ }^{42} \mathrm{Zu}$ Fällen, in denen stattdessen - $d$ - steht, s. EK ̈̈ (77f.) und für weitere Schwundfälle EKÄ (78ff.)
} 
e:15 -nik ist Ableitungssuffix (KASIK 2015: 263)

est. aednik 'Gärtner', vgl. est. aed 'Garten'

e:16 -e ist Ableitungssuffix (KASIK 2015: 272ff.; EKG 534ff.)

est. pääse, gen. pääsme 'Eintrittskarte, Ticket', vgl. est. pääsema 'gelangen, geraten, eindringen'

e:17 -ne ist Ableitung (KASIK 2015: 348)

est. vesine 'wässrig', vgl. vesi 'Wasser'

e:18 $-i$ - ist Ableitungssuffix (LAANEST 1982: 275)

est. kaapima 'schaben', vgl. est. kaapama 'einmal schaben'

\subsubsection{Lautwandel im Wotischen}

$\mathrm{w}: 1 \quad *_{-} e_{-}>-\tilde{o}-$ in nichtersten Silben

wot. õpõa 'Silber' < fosfi. *šope- $\delta a$

w:2 *-euv- > -evv- (VKÄ: 137)

wot. pеуvалла (<* * putalla) 'trockene Witterung, schönes Wetter-ADESs.'

$\mathrm{w}: 3 \quad *_{-} u->-i-(\mathrm{VKÄ:} 137)$

wot. neizen 'anfangen, (auf)steigen'-1SGPRÄS

w:4 gelegentlich $*_{-} o>-u$ im Auslaut (VKÄ 142)

wot. лukku 'Schloss, Verschluss' fi. lukko id.

w:5 $\quad ? * \tilde{o}>i$ vor $* k l$ (russ. Einfluss) (VIITSO 1978b: 94)

wot. nigla 'Nadel' est. nõel id.

$\mathrm{w}: 6 \quad * k>t \check{s}(\mathrm{VK} \ddot{\mathrm{A}} 22)$

wot. itšä 'Alter' fi. ikä id.

$\mathrm{w}: 7 \quad *_{-s t-}>-s s-(\mathrm{VKÄ} \mathrm{26f.)}$

wot. vassen 'neu' (<*vastainen $)$

$\mathrm{w}: 8 \quad *_{-k s-}>-h s-(\mathrm{VK} \ddot{\mathrm{A}}: 36)$

wot. mahsā 'zahlen, bezahlen' fi. maksaa id. 
w:9 *ts vor offenen Silben > tts (VKÄ 42)

wot. vattsa 'Bauch' : vatsassa id.-SGELAT

w:10 Schwund des Dentals nach Vokal der unbetonten Silbe (VKÄ 69; LAANEST 1982: $205)^{43}$

wot. kõrkõa 'hoch' < *korke- $\delta a$; wot. ẹpea 'Silber' < fosfi. *šope- $\delta a$

w:11 *h-> Ø im Wortanlaut (VKÄ 83)

wot. ampāD 'Zähne' fi. hampaat id.

w:12 Schwund von $*_{-} h$ - nach Diphthong mit $-u$ - als zweitem Element, daraufhin $*_{-} u->-v-$ (VKÄ 85)

wot. javan (<*jau-an < jauhan) 'mahlen-1SGINDPRÄS'

w:13 Vokalisierung von $*_{n}$ in Verbindung $*_{n s} *_{-} n s^{-}>-V s^{-}$im Auslaut der ersten Silbe (VKÄ 92f.)

wot. pẹzzaz 'Busch' fi. pensas id.; wot. kẹzz (< konsa) 'wann, wenn, als'

$\mathrm{w}: 14 *-n t->-t-(\mathrm{VKÄ} \mathrm{94)}$

wot. avato 'Eisloch' fi. avanto id.

$\mathrm{W}: 15 \quad *_{-} m p->p(\mathrm{VK} \ddot{\mathrm{A}} 106)$

wot. sūrepi 'groß-KoMPSG' fi. suurempi id.

$\mathrm{w}: 16 \quad(*-s j->) *-z j->z z z ́(\mathrm{VKÄ} \mathrm{115)}$

wot. aźźa $(<a s j a)$ 'Sache'

$\mathrm{w}: 17 *-u v->-v v-(\mathrm{VKÄ} 118)$

wot. nevvoa 'jm. beraten, Ratschläge geben' fi. neuvoa id.

w:18 -o ist Ableitungssuffix (ARISTE 1968: 115)

wot. nītto 'Wiese' (< wot. nīttë̈ '(Rasen) mähen'

w:19 $-z$ ist Ableitung (ARISTE 1968: 115)

wot. kagлиz 'Kragen; Halstuch' ( < wot. kagла 'Nacken')

w:20 $-u$ - ist Ableitungssuffix (ARISTE 1968: 119)

wot. raskeun 'absteigen, herunterkommen-1SGPRÄs' (< raskęa 'lassen')

\footnotetext{
${ }^{43}$ Für weitere Schwundfälle, s. VKÄ (68-71).
} 
$\mathrm{w}: 21-u D$ ist Ableitungssuffix (LAANEST 1982: 217)

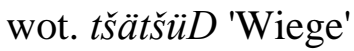

\subsubsection{Lautwandel im Livischen}

1:1 Vertretung von $-V$ - in langer sonorer Silbe durch $-V-\sim-\bar{V}$ - (PosTI 1942: 4-22)

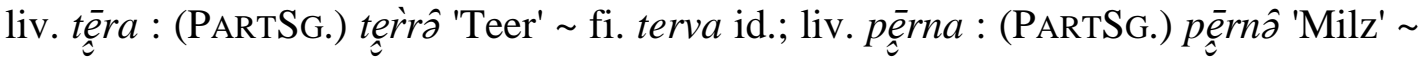
fi. perna id.

1:2 $*_{-}$- - der ersten Silbe > -иo- (Posti 1942: 6ff.); *-e- der ersten Silbe zu -ie-, im Wortanlaut zu je- (ebd. 19)

liv. $k^{u}$ odà 'Haus, Küche [...]' fi. kota; liv. thotà 'wahr-PARTSG' fi. totta id.;

liv. t'erà 'Schneide, Ährenspitze' fi. terä id.; liv. l'ep̀à 'Erle' fi. leppä id.

1:3 epenthetisches $-u$ - aus der zweiten Silbe verhindert $*_{-}-o_{-}>-$-

liv. sǒgdâ, auch: sǒugdâ $(<* \operatorname{sogu\delta a<*sokęa)~'blind'~~~fi.~sokea~id.~}$

1:4 *-o- in langen sonoren Silben durch $-^{u} O-\sim$ liv. kùona : (PARTSG) kuǹnâ 'Frosch' fi. konna id.; kùora : (PARTSG) kuor̀râ 'Ohr' $\sim$ fi. korva id.

1:5 Wortanlautendes $*$ - durch livO. $v o-\sim v \bar{Q}-$, livW. $v^{u} o, v u ̀ o$ vertreten (PosTI 1942: 12) liv. vořžn, vogrž : (PARTSG) vọrta 'Stange, Hühnerstange' fi. orsi id.

1:6 $\quad *_{-o-}>-u$ - vor $-m$ - (PosTI 1942: 13)

liv. um̄blâ 'nähen' fi. ommella id.

1:7 $\quad *_{-} \ddot{u}->-\tilde{o}-$ vor $-v-($ POSTI 1942: 15f.)

liv. tềvà 'tief' fi. syvä id.; liv. têِّu(v) 'breites Ende am Stamm oder Balken' fi. tyvi 'unteres dickes Ende (bes. bei Baumstamm)'

1:8 *-oi- der ersten Silbe vertreten durch -ùoi- - ${ }^{u}$ ò̀- im Ostlivischen (POSTI 1942: 31f.) liv. tùoista 'andere, zweite' fi. toinen id.

1:9 $\quad *-V i->-V u-$ vor $-v-($ PosTI 1942: 32)

liv. keùù 'Birke' fi. koivu id.

1:10 Vertretung von *-oi- durch -ùoi- -ò̀- nach $p$ - und $m$ - (POSTI 1942: 32)

livW. mu oizâ 'Herrengut, Hof' livO. moìzâ id. fi. moisio id. 
1:11 *-oi- > -e i- vor oder nach -v- (PosTI 1942: 33)

livO. vęì B 'können'-3SGINDPRÄS fi. voi id.

1:12 *-au-vertreten durch -ō- -où- (PosTI 1942: 35f.)

liv. sōna 'Badestube' : soùnâ id.-PARTSG fi. sauna 'Sauna'

1:13 - -

liv. sè̀

1:14 Vertretung von *-ou- durch -ō- -où- (POSTI 1942: 37)

liv. joùdâ 'gelangen, können; Kraft, Muße haben' : jōdaB id.-3SGPR fi. joutaa id.

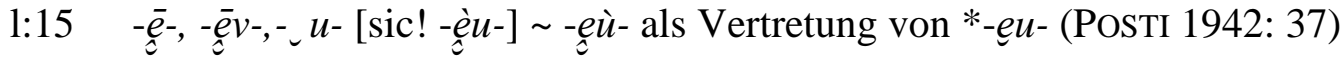

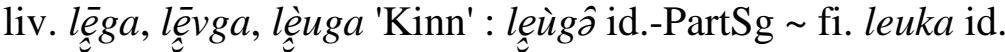

1:16 $\quad *_{-i-}>-\tilde{o}_{-}$-ausgelöst durch - $u$ - (POSTI 1942: 38f.)

liv. lè̀̆glâ 'stolpern, gleiten; fallen, springen' fi. liukua id.

1:17 früher Verlust des vordervokalischen Charakters von *-ä- in nichterster Silbe (POSTI 1942: 42f.)

liv. siè̀à 'Schmied' fi. seppä id.; liv. kätà 'Hand-PARTSG' fi. kättä id.

1:18 $*_{-} a->-i$ - in nichterster Silbe, wenn ein palatalisierter Konsonant vorausgegangen ist (PosTI 1942: 44)

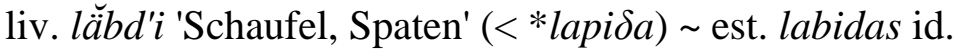

1:19 - $-\hat{\partial}$ - in nichterster Silbe sekundär

liv. vęrgâ 'Netz' < *verkka

z.B. weiter hinten als in der zweiten Silbe immer $*_{-} a->-\hat{\partial}$ - (POSTI 1942: 44)

liv. $k^{u} o \bar{r} d \hat{\partial}(<* k o r k e \varrho a)$ 'hoch' $\sim$ fi. korkea id.; liv. lăgda $(<* l a k e \varrho \delta a)$ 'flach, wüst' fi. lakea id.

z.B. $*_{-} a->-\hat{\partial}-$ nach kurzem Vokal, auf den Verbindung von Klusil und Liquida folgt (PosTi 1942: 44)

livW. kăglâ (späturfi. *kakla) 'Hals' fi. kaula id.; liv. ăddârz 'Pflug' fi. aura id.

z.B. $*_{-} a->-\tilde{o}$ - in zweiter Wortsilbe vor (ursprünglichem oder vor -ns- oder silbenschließenden - $h$ - entstandenem) langem Vokal in der ersten (PosTi 1942: 43) liv. vềrâz 'fremd' fi. vieras id.; liv. pē̌zâzz (<* pensas) 'Busch' fi. pensas id.; liv.

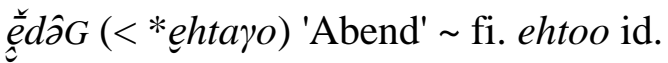


z.B. $*_{-} u->-\hat{\partial}^{-}$in zweiter Wortsilbe in den gleichen Fällen, in denen auch $-a->-\hat{\partial}-$ (PosTi 1942: 48)

liv. е̌ge $g \hat{~}(<* h e ̨ h k u-)$ 'glühen, schmerzen' fi. hehkua id.

z.B. $*_{-} u->-\hat{\partial}-$ in zweiter Wortsilbe (livO), wenn in der ersten ein gelängter Vokal oder Diphthong steht (PosTi 1942: 48f.)

liv. līndâD 'Vogel'-NomPL fi. lintu 'Vogel'

1:20 $*_{-o-}>-u$ - in nichterster Silbe (POSTI 1942: 48)

liv. mõtūks 'Auerhuhn, -hahn' fi. mettos id.

1:21 Apokope (Posti 1942: 62)

liv. kan̄D 'Baumstumpf' fi. kanto id.

1:22 -â im Wortauslaut vertritt Vokalkontraktion (PosTı 1942: 70ff.)

liv. kirrrâ 'Axt'-GENSG (<*kirvehen $) \sim$ fi. kirveen id.

1:23 Synkope unabhängig von der Länge der vorausgehenden Silbe und davon, ob die geschwundene Silbe offen oder geschlossen war (POSTI 1942: 76ff.)

liv. $u \bar{l} d \hat{\partial}(<* u \overline{l g} d \hat{a}<* u l k o \delta a)$ 'außerhalb, von außen' fi. ulkoa id.; liv. kǔltầ $(<* k u$ -

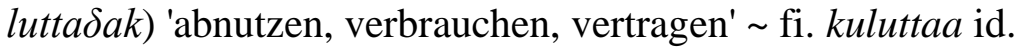

1:24 Epenthese von - $i$ - in zweiter Silbe (PosTI 1942: 102ff.)

livW. tốiB <*topi) 'Krankheit'

1:25 *-iu- > jęu- (POSTI 1942: 125)

liv. ję̀uG 'Sand' fi. hiukka 'Sandkorn, Stückchen; Partikel [...]'

1:26 *-üv->-ęv-(POSTI 1942: 128)

liv. jęvà̀ 'gut' $(<* \ddot{u} v \ddot{a}<* h \ddot{u} v \ddot{a})$

1:27 *h-> Ø im Wortanlaut (POSTI 1942: 145)

liv. $a \bar{l} G$ 'Scheit, Holzscheit' fi. halko id.

1:28 *j-im Anlaut entstanden durch „Brechung“ von - ̈̈- (POSTI 1942: 123-129, 146)

liv. jểà̀ 'gut' $(<* \ddot{u} v \ddot{a}<* h \ddot{u} v \ddot{a})$

1:29 Ausfall von $*$ - $h$ - in Verbindung $-h k$ - (Posti 1942: 160)

liv. tŭgâD 'Asche'-PL $(<*$ tuhkat $) \sim$ fi. tuhka id. 
1:30 $\quad *_{-} z^{-}>-\check{z}-$ vor - $i-($ PosTI 1942: 233)

liv. věžž, věiž 'Wasser' (<*vesi)

1:31 *-sj- vertreten durch -̌́- (POSTI 1942: 236)

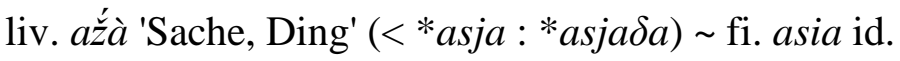

1:32 Schwund von *-h- nach Nasal oder Liquida (PosTi 1942: 243)

liv. vanà (<*vanha) 'alt' fi. vanha id.; liv. věsl (<*velho) 'Hexe' fi. velho id.

1:33 Schwund von *- $h$ - im Silbenauslaut der ersten Silbe, stattdessen Auftreten von langem Vokal oder Diphthong mit Stoßton (PosTi 1942: 243f.)

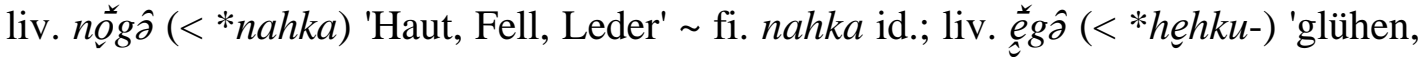
schmerzen' fi. hehkua id.

1:34 Vokalisierung von $*_{-} n$ - in Verbindung $*^{*} n s: *_{-} n s->-V s-$ nach der hauptbetonten Silbe.

Dadurch wird eine Vokallängung des vorausgehenden Vokals verursacht (POSTI 1942: 253)

liv. pẹ̃zzâz (<*pensas) 'Busch' fi. pensas id.; liv. mōš̌kâz (<*mansikka-) 'Erdbeere' $\sim$ fi. mansikka id.

1:35 $*_{-} v->-u$ - im Silbenauslaut vor geschwundenem Vokal (Posti 1942: 265)

liv. kěururâ 'krumm, schief' fi. kovera 'konkav, ausgehöhlt'

1:36 *-lv->-ll-(POSTI 1942: 266)

liv. tōla 'Winter' fi. talvi id.

1:37 Metathese in Verbindung *-hv-(PosTI 1942: 266)

liv. ǒunôD 'Barsch'-NomPL (<*avenet < *auhenet $<*$ ahvenet $) \sim$ fi. ahvenet id.

1:38 Schwund von Vokal in der Position $-n V n-($ Posti 1942: 311)

liv. ěsbbi 'Pferd' (< ębbin < *hępoinenn)

1:39 Zusammenfall und Schwund von *-nn- (PosTI 1942: 311)

liv. ęsbbi 'Pferd' (< ębbin < *hepoinen)

1:40 Stoßton, da anlautender Konsonant der Folgesilbe durch Apokope, Synkope oder Gemination in den Silbenauslaut gekommen ist (POSTI 1942: 318)

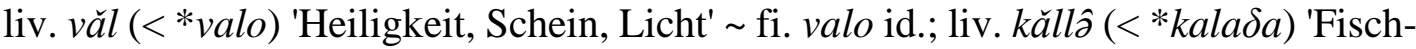
PARTSG' fi. kalaa id.; liv. pǔnni (<* *unainenn) 'rot' fi. punainen id. 
1:41 Stoßton wegen Verbindung von Klusil und Liquida an Grenze der ersten und zweiten Silbe (PosTI 1942: 318)

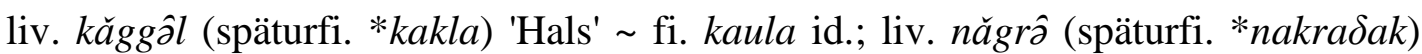
'lachen' fi. nauraa id.

1:42 Stoßton, da an der ersten Silbengrenze -s- und Halbvokal vorliegen und der Vokal in der zweiten Silbe geschwunden oder kontrahiert ist (POSTI 1942: 318)

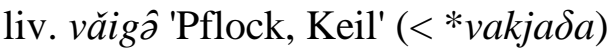

1:43 Stoßton aus - $h$ - des Anlauts der Folgesilbe entstanden (Posti 1942: 318f.)

liv. rŏ $(<*$ raha) 'Geld' fi. raha id.; liv. lǐebi $(<*$ lihaßainen $)$ 'fett, dick' $\sim$ fi. lihava id.

1:44 Stoßton nach einer hauptbetonten Silbe, die ursprünglich auf $*$ - $h$ - ausgelautet ist (Posti 1942: 319)

liv. tŭg$\partial \hat{D}$ 'Asche'-PL $(<* t u h k a t) \sim$ fi. tuhka id.

1:45 $\quad-i$ ist Ableitung (vgl. LAANEST 1982: 206f.)

liv. roùdi 'eisern'

1:46 -lâ ist Ableitungssuffix (LAANEST 1982: 274)

liv. eit̀â 'hin und her werfen'

\subsubsection{Lautwandel im Südestnischen}

eS:1 $\quad *_{-o-}>-u$ - vor $-h-($ EK ̈̈ 132)

estS. whak 'Distel' est. ohakas id.

eS:2 $\quad *_{-}-{ }_{-}>-u$ - vor $-m$ - (KEEM 2002: 33)

estS. um̀Blęmma 'nähen' fi. ommella id.

eS:3 $*_{-} o->-u$ - in nichterster Silbe (vgl. KEEM 2002: 34)

estS. mõtus 'Auerhuhn, -hahn' fi. mettos id.

eS:4 estS. $a i \sim$ est. ei (KEEM 2002: 36)

estS. hain 'Heu' est. hein, gen. - $a$ id.

eS:5 Assimilation des zweiten Diphthongbestandteils an den ersten (KEEM 2002: 36)

estS. kāvęDu' 'gegraben' est. kaevatud id. 
eS:6 Apokope (EK ̈̈ 196)

estS. küлm, gen. kü̆mà̀ 'kalt' fi. kylmä id.

eS:7 Synkope (EKÄ 196, KeEM 2002: 35)

estS. op̄ma 'lernen' est. óppima id.; estS. laùlva 'singen-3Pl' est. laulavad id.

$\mathrm{eS}: 8 \quad-u-\sim-v-$

estS. kõvve 'Donner'

eS:9 - $\tilde{o}$ - in nichtersten Silben sekundär

z.B. $*_{-} e_{-}>-\tilde{o}-$ in nichterster Silbe

estS. hõpo 'Silber' fi. hopea id.

eS:10 *-tk- > -tsk- (EKÄ 32)

estS. katsk 'Pest', vgl. est. katk id.

eS:11 Schwund von intervokalischem $*_{-j-}$ (vgl. auch EKÄ 114)

estS. mõo|ma, II. Infinitiv -daq 'beeinflussen, wirken'

eS:12 Schwund von intervokalischem *-v- (vgl. EKÄ 116)

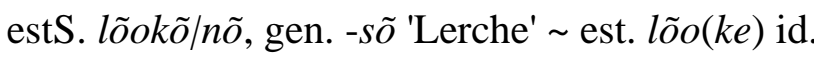

eS:13 -ss im Silbenauslaut (KEEM 1997: 7)

estS. kotuss 'Ort, Stelle'

eS:14 *-ks- > -ss-, -z-, -s'- (KEEM 2002: 25)

estS. $u s^{\sigma}$ 'Tür' est. $u k s$ id.

eS:15 $\quad *_{-t s-}>-d s-$ nach Langvokal in erster Silbe (KEEM 2002: 26)

estS. lõôts, gen. lõodsa 'Blasebalg'

eS:16 Gemination (KEEM 2002: 27)

estS. jämme, gen. jämeDä 'dick, stark, grob' est. jäme id.

eS:17 Metathese des intervokalischen $*-h$ - (KEEM 2002: 29)

estS. jõ $h v$ ', gen. jõhvi 'Pferdehaar, Rosshaar'

eS:18 *-ht- >-tt- (KEEM 2002: 30)

estS. $k \tilde{o t} \mid t$, gen. $-u$ 'Bauch' est. kõh|t, gen. $-u$ id.

eS:19 Metathese *-lh- > - hl- (KEEM 2002: 31)

estS. võhl, gen. võhlu 'Zauber' fi. velho 'Hexenmeister, Hexe, Wunderheiler' 
eS:20 Palatalisation

z.B. durch Einfluss von $-i$ - oder Schwund von $*_{-j}-$ oder $*_{-} i$ - (KEEM 2002: 25, 32)

estS. pechi, gen. pęha 'Boden, Grund'

z.B. des auslautenden Konsonanten in dreisilbigen Wörtern mit $A$ - oder $E$-Stamm, wenn Apokope oder Gemination vorliegt (KEEM 2002: 32)

estS. kü̈päŕ, gen. küBärä 'Hut'

eS:21 le- ist Ableitungssuffix (LAANEST 1982: 274)

estS. pil̆'viskellemä 'sich abwechselnd bewölken und aufklären'

eS:22 $-u$ - ist Ableitung (LAANEST 1982: 282)

estS. kak̀kuma 'entzweigehen, sich zerschleißen'

eS:23 für -nik ist wohl eine Ableitung ansetzbar (vgl. est.)

estS. sõnnik Mist, Dünger' est. sõnnik, gen. $-u$ id.

eS:24 -o ist wohl Ableitung (vgl. wot.)

estS. võso 'Schössling, Spross'

\subsection{Das Korpus}

\subsubsection{Kurzvokale}

1 est. hõbe 'Silber' wot. õpõa id. liv. ó'bdõ id. estS. hõpõ id.

fi. hopea 'Silber'

fosfi. *šope- $\delta a$ 'weich' (SSA I: 172); osfi.-lp. (EES 81f.; EEW II: 415)

Zum Ableitungssuffix $-\delta A$ und seinen einzelsprachlichen Vertretungen s. LAANEST (1982: 205).

est: e:3, e:4

wot. w:1, w:10, w:11

liv. 1:19, 1:23, 1:27, 1:40

estS: eS:9

2 est. hõlm, gen. - $a$ 'Rockschoß' $\sim$ wot. õlma id. liv. õlma id. estS. hõlm, gen. hõlma id.

fi. helma '(Rock)schoß, Saum'

urfi. *helma (LÄGLOS I: 92) 
? < germ. (SSA I: 154); > germ. (EES 82); osfi. (EEW II: 423); germ. Lehnwort (LÄGLOS I: 92)

est: e:3

wot: w:11

liv: $1: 1,1: 27$

estS: eS:6

3 est. hõl|p, gen. -bu 'Leichtigkeit' estS. hõlp, gen. hõlbu id. | wot. helppo 'leicht, mühelos'

fi. helppo 'leicht; locker'

urfi. *helppo (LÄGLOS I: 93)

? < germ. (SSA I: 154f.); < urgerm. (EES 82); osfi. *helppo oder ? < germ. (EEW II: 423ff.); germ. Lehnwort (LÄGLOS I: 93)

est: e:2, e:3

wot: w:11

estS: eS:6

4 est. horra-(in Zuss. mit hõrasilm 'starres Auge') wot. õrahtaa 'vor dem Melken Milch ins Euter sammeln' liv. ? egrā 'Geifer von brünstigen Tieren' estS. horra(s)silm 'schielendes Auge'

fi. hera 'klare Flüssigkeit, Molke'

urfi. *hera [<*šera] (LÄGLOS I: 96)

SSA (I: 157) erwägt ein mögliches urgerm. (< indoeur.) Lehnwort. Die Verbindung des liv. Belegs und der dort genannten Variante mit dem Estnischen gilt in SSA und nach KETTUNEN (LW 51) als unsicher.; kein Eintrag in EES; osfi. (EEW II: 426); ? germ. oder älteres Lehnwort (LÄGLOS I: 96)

wot: w:11

liv: $1: 27$

5 est. hõrak- (in Zuss.) 'Johannisbeere' liv. evrā-māráa 'rote Johannisbeere' estS. hõrak id.

fi. herukka 'Johannisbeere'

SSA (I: 159) setzt eine Ableitung der osfi. Entsprechungen vom gleichen Stamm wie fi. herua 'triefen', hera 'klare Flüssigkeit, Molke' an. Diese werden dort nicht weiter behandelt.; EES (83) bezeichnet den Beleg als osfi. Stamm, welcher der gleiche wie zu est. hõrasilm 'starres Auge' sein könne. Die Zusammengehörigkeit ist ungesichert.; 
MÄGISTE (EEW II: 427f.) setzt die est. Entsprechung bei hõrak-mari 'schwarze Johannisbeere' an und macht keine Herkunftsangaben.

liv: $1: 27$

6 est. $h \tilde{o r} \mid k$, gen. -gu 'appetitlich' | wot. erkku 'Delikatesse'

fi. herkku 'Delikatesse, Leibgericht'

Laut SSA (I: 158) in einigen osfi. Sprachen belegt; osfi. (EES 83); keine Herkunftsangabe in EEW (II: 429)

wot: w:11

7 est. (h)ôrn, gen. - $a$ 'zart, liebevoll' estS. õrn, gen. õrna id. | liv. vörna 'sanft, zart, liebevoll'

fi. hornea 'lau; kühl; unheimlich'

Laut SSA (I: 174) in einigen osfi. Sprachen belegt; osfi. (EES 627; EEW XII: 4034f.)

est: e:3

liv: 1:5, 1:27

estS: eS:6

8 est. jõgi, gen.jõe 'Fluss' wot. jõtši, gen. jõgõõ id. estS. jõgi, gen. jõõ id. | liv. jo'ug id.

fi. joki 'Fluss'

Laut SSA (I: 240) in einigen fiugr. Sprachen belegt; fiugr. (EES 103) keine Herkunftsangaben in EEW (II: 580f.)

liv: 1:24, 1:40; -u- in jo'ug wahrscheinlich aus jogùd 'Fluss-NoMPL' (LW 92); PosTI (1942: 8f.) bezeichnet das Wort als analoge Form zu 1:3.

estS: eS:9

9 wot. jõka 'jeder' | ? est. iga id. ? liv. jeka id. estS. ? egä; ogga id.

fi. joka 'jeder'

SSA (I: 239) erwägt eine Zusammengehörigkeit von fi. und wot. Ferner werden die est. und liv. Varianten zusammengestellt. Für letztere wird ein Ursprung <*ekä, welches auf den Pronominalstamm $e$ - zurückgehe, angesetzt. Dies trete nur ein, wenn das $j$ - in der Verbindung jo- nicht einen Lautwandel -o- > - $e$ - verursacht habe.; EES (88) erwägt einen osfi.-wolg. Stamm. Die osfi-lp. Entsprechungen seien von *jo- mit der Partikel

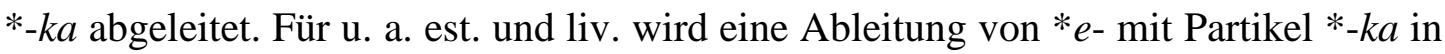
Betracht gezogen.; MÄGISTE (EEW II: 483f.) setzt ebenso * ekä für est. und liv. an. liv: $1: 2$ 
10 est. jõlkuda 'hin und her gehen' estS. .jõlkma, II. Infinitiv jõlkuq id.

fi. jolkata, jolkuttaa 'langsam laufen, gehen, traben'

SSA (I: 241) setzt eine deskriptive Wortgruppe an. Die estnischen Entsprechungen werden dort als dial. angesetzt.; osfi. onomat. (EES 103; EEW II:583)

est: e:12

estS: eS:7, eS:22

11 liv. jõva 'gut' | est. hüva id. wot. $\ddot{u} v \ddot{a} \mathrm{id}$. estS. hä̈̈ hü(v) ä id.

fi. hyvä 'gut'

*fosfi. šüvä (SSA I: 201)

fosfi. (SSA I: 201); EES (86) setzt einen osfi.-md. oder fiugr. Stamm an und erwägt eine Entwicklung von -ä̈̈- > -ea- für die dial. Varianten. Das intervokalische $-v$ - sei zudem geschwunden.; MäGISTE (EEW I: 311) vermutet einen osfi.(? -fiugr.) Stamm und setzt für die est. Variante hää die Entwicklung hää < hüä < hüvä an.

wot: w:11

liv: 1:7, 1:26, 1:28

estS: eS:12

12 est. $k \tilde{o} h \mid t$, gen. - $u$ 'Bauch' wot. kõhu|z, gen. -hsõo 'Taille' estS. kõt|t, gen. - $u$ 'Bauch' fi. kohtu 'Gebärmutter'

Laut SSA (I: 383) in einigen osfi. Sprachen belegt; osfi. (EES 199); MÄGISTE (EEW IV: 1085) setzt fiugr. *kokt- > kõht $\sim$ kõtt an.

est: e:3; - $u$ wird von UEW (II: 670) als Ableitung angesetzt

wot: ? w: 19

estS: eS:18

13 wot. kõhta 'Ort, Stelle' | est. koht, gen. koha id. liv. ku'odi 'gerade, ehrlich' estS. kuht, gen. koha kuha 'Ort, Stelle'

fi. kohta 'Stelle, (Zeit)punkt; (als Partikel) sofort, bald, gleich'

Laut SSA (I: 383) in allen osfi. Sprachen belegt; EES (170) erwägt einen osfi. oder osfi.-lp. Stamm oder eine mögliche Entlehnung, ? < balt. *kokta; ? ural. kohta $<*$ kokta (EEW III: 894)

est: e:3

liv: $1: 2,1: 18^{44}, 1: 33$

\footnotetext{
${ }^{44}$ Die Palatalität des $d$ im livischen Beispiel zeigt sich in KETTUNENS (LW 163) Transkription: $k u$ 'od'i.
} 
estS: eS:1, eS:6

14 est. kõla 'Gerät zum Weben eines Gürtels' ? liv. kõlā 'Bogen, Gewölbe, Bucht' estS. kõla 'Gerät zum Weben eines Gürtels'

fi. kela 'Haspel, Winde; Spule'

SSA (I: 340) erwägt > balt., est. sei dial. Variante.; < balt. (EES 199; EEW IV: 1089)

15 est. kõle 'trocken' wot. kõla 'trocken, kalt' liv. kõ'l id. estS. kõlló 'trocken'

fi. kolea 'kühl; rauh; düster; uneben'

Laut SSA (I: 389) in einigen osfi. Sprachen belegt; EES (200) setzt eine Variante von est. kole 'scheußlich, grässlich, unheimlich' an, welche ein osfi.-lp. Stamm sei (ebd. 172); osfi. (EEW IV: 1091)

Als Stamm ist *kole- $\delta a$ anzusetzen. Zum Ableitungssuffix $-\delta A$ und seinen einzelsprachlichen Vertretungen s. LAANEST (1982: 205).

est: e:3, e:4

wot: w:10

liv: 1:21, 1:23, 1:40; ? < est. (LW 118)

estS: eS:6, eS:9, eS:16

16 estS. kõlla|nõ, gen. -dsõ 'gelb' [estN. kold, gen. kolla 'Bärlapp (eine gelbe Farbe gebende Pflanze); Dotter, Eigelb', kollane 'gelb'] wot. kõlta id. | liv. ? keldarikki 'Sumpfdotterblume'

fi. kelta 'gelb; Name verschiedener Pflanzen'

< balt. (SSA I: 342; EES 172; EEW III: 908)

est: Für das Estnische ist die estS. Variante als einschlägig anzusetzen. Laut UNIVERE (1986: 69) haben die estS. Dialekte den Laut - $\tilde{o}$ - bewahrt, während in estN. -o- vorliegt (vgl. auch KEEM 2002: 33). Ein Lautwandel $*_{-} e->-\tilde{o}->-o$ - ist für estN. wohl ansetzbar. Für eine derartige Entwicklung sprechen zudem die anderen Vertretungen, vgl. auch kar. kelta 'gewöhnlicher Flachbärlapp', ing. keltain 'gelb' (SSA I: 342).

estS: eS:9

17 wot. kõlmõD 'drei' | est. kolm, gen. -e id. liv. kuolm id. estS. kolm, gen. kolmõ id. fi. kolme 'drei'

Laut SSA (I: 391f.) in allen fiugr. Sprachen belegt; fiugr. (EES 173); MÄGISTE (EEW III: 920f.) erwägt fiugr. Stamm *kolmi.

est: e:3

wot: w:1 
liv: $1: 2,1: 21$

estS: eS:6, eS:9

18 est. kõlvata 'taugen' wot. kõlvata id. liv. kõlbõ id. estS. .kõlbama id.

fi. kelvata 'taugen'

urfi. *kelpa- (LÄGLOS II: 79)

< urgerm. bzw. vorgerm. (SSA I: 342); urgerm. (EES 199); osfi. (EEW IV: 1090); germ. Lehnwort (LÄGLOS II: 79f.)

liv. 1:19, 1:23

19 est. kõmada 'dumpf tönen'

fi. komata 'dumpf, hohl tönen, donnern'

SSA (I: 393) setzt einen onomat. Stamm an. Dort wird fi. komata zu est. kómiseda 'dröhnen, dumpf schallen' gestellt, welches als abgeleitet anzusetzen ist (vgl. dazu auch KASIK 2015: 175); osfi. onomat. (EES 200; EEW IV: 1095)

20 est. kõmmeld(i) 'Hohlmesser, Hohleisen', kõveldi id., kõmmeldada 'aushöhlen' fi. koveli 'Hohleisen'

Laut SSA (I: 415) in einigen osfi. Sprachen und im Lp. belegt; EES (200) erwägt als Hauptvariante dieses Beispiels est. kõmmeldi und bezeichnet es als osfi.-lp. Stamm. Weitere Formen wie est. kõveldi und koveldi werden dort als dial. Formen angesetzt.; osfi.-lp. (EEW IV: 1096). Eine mögliche Beeinflussung durch den Stamm est. kumm 'Gewölbe, Wölbung' halten EES (200) und MÄGISTE (IV: 1096) für wahrscheinlich. Dort wird est. kõmmeldi nicht weiter behandelt.

21 est. kõmpida 'einherschreiten, schwerfällig gehen' estS. .kõmp'ma, II. Infinitiv .kõmpiq; .kõmpsíma, II. Infinitiv .kõmpsiq id.

fi. kompia 'kriechen'

Möglicherweise deskriptiv (SSA I: 395); EES (200) bezeichnet den Stamm als osfi. onomat. und erwägt eine Variante von est. kompama 'anfühlen, anfassen' und komberdama 'holpern, stolpern, humpeln'. Diese sind jedoch nicht gesichert.; est.(-?fi.) onomat. (EEW IV: 1096)

estS: eS:7, eS:20

22 est. kõndida 'gehen, spazieren' wot. kõntaassa 'hin und zurück gehen, spazieren' estS. .kõnd'ma, II. Infinitiv .kõndiq 'gehen, spazieren'

fi. kontata 'kriechen' 
SSA (I: 397) erwägt eine Verbindung zu fi. kontti 'Bein, Unterschenkel(knochen)'. Dort wird est. kõndida nicht weiter behandelt.; EES (200f.) geht von einer osfi. Form und einer Variante von est. kont 'Knochen' oder est. kand 'Kante, Saum; Landstrich' aus. Dort werden est. kõndida und Entsprechungen nicht besprochen.; osfi. (EEW IV: 1097). Eine Verbindung zu wot. kõntaassa wird in den etymologischen Wörterbüchern nicht angesetzt, es ist jedoch dazuzustellen.

estS: eS:7, eS:20

23 est. kõn|t, gen. - di 'Steifigkeit, Verkrüpplung'

fi. kontta '(durch Kälte verursachte) Starrheit'

Laut SSA (I: 398) in einigen osfi. Sprachen belegt; kein Eintrag in EES; osfi.(?-lp.) (EEW IV: 1099)

est: e:3

24 est. $k \tilde{o r} \mid b$, gen. -ve/be 'Urwald'

fi. korpi 'tiefer (Fichten)wald; Urwald; Bruchwald; Wildnis'

Laut SSA (I: 405) in einigen osfi. Sprachen belegt; EES (201) setzt den Stamm als osfi. an und erwägt die Zusammenstellung mit dem liv. Ortsnamen Kūorbõd.; osfi. (? fiugr.samoj.) (EEW IV: 1100)

est: e:3

25 est. kõrbeda 'anbrennen' estS. .kõrbõma, II. Infinitiv kõrbõdaq kõrvõdaq id. $\mid \sim$ liv. kuorbõ id.

fi. korventaa 'abbrennen, (ver)sengen, erhitzen'

Laut SSA (I: 408) in allen osfi. und einigen weiteren fiugr. Sprachen belegt; osfi.-md. (EES 201); MÄGISTE (EEW IV: 1100) bezieht sich auf die Variante est. $k \tilde{o} r \mid b$, gen. -ve 'Versengen, Brennen, Anbrennen', macht jedoch keine Herkunftsangabe.

liv: $1: 2,1: 19,1: 23$

estS: eS:9

26 estS. kõrd, gen. kõrra 'Ordnung, Reihe' [estN. kord, gen. korra id.] wot. kõrta id. liv. kõr $r d a$ id.

fi. kerta 'Mal, Schicht'

urfi. *kerta (LÄGLOS II: 86)

SSA (I: 347f.) nennt zwei Entlehnungsmöglichkeiten: < balt. oder urgerm.; < balt. (EES 178; EEW II: 952f.); ? germ. Lehnwort oder balt. (LÄGLOS II: 86f.) 
est: Für das Estnische ist die estS. Variante als einschlägig anzusetzen. Laut UNIVERE (1986: 69) haben die estS. Dialekte den Laut -õ- bewahrt, während in estN. -o- vorliegt (vgl. auch KEEM 2002: 33). Ein Lautwandel $*_{-} e->-\tilde{o}_{-}>--o$ - ist für estN. wohl ansetzbar. Für eine derartige Entwicklung sprechen zudem die anderen Vertretungen, vgl. auch kar. kerta 'Mal, Schicht', weps. kerd id. (SSA I: 347).

est: e:3

liv. 1:1

estS: eS:6

27 est. kõrend, gen. -i 'Stange' wot. kõrõta 'Tragholz, Schulterjoch' estS. kõreńd 'Stange'

fi. korento '(Trag)stange [...]'

SSA (I: 402) erwägt, dass fi. korento möglicherweise auf den gleichen Stamm zurückgehe wie fi. korelo 'Schürhaken' und fi. korilo id., auch: '(Trag)stange, [...]; Libelle'.; EES (201) erwägt eine Ableitung vom gleichen Stamm wie est. kõre 'lang und gerade', welcher osfi. sei; MäGISTE (EEW IV: 1102) bezeichnet den Stamm als osfi-md. (oder ? fiugr.) und setzt ebenfalls eine Verbindung zu est. kõre an.

est: e:3

wot: w:1

estS: eS:6, eS:20

28 est. kõrge 'hoch' wot. kõrkõa id. | liv. kuordõ id. estS. .korgõ 'hoch'

fi. korkea 'hoch'

Laut SSA (I: 403) in allen osfi. Sprachen belegt; osfi. (EES 201f.); osfi.-lp. (? fiugr.) (EEW IV: 1103)

Als Stamm ist *korke- $\delta a$ anzusetzen. Zum Ableitungssuffix $-\delta A$ und seinen einzelsprachlichen Vertretungen s. LAANEST (1982: 205)

est: e:3, e:4

wot: w:1; w:10

liv: $1: 2,1: 19,1: 23$

estS: eS:6, eS:9

29 wot. kõrjata 'verstecken, (sich) bedecken; sammeln, pflücken' | est. korjata 'sammeln, pflücken, suchen, [...]' liv. kuor'ŕs 'sammeln, pflücken' estS. .korjama, II. Infinitiv koradaq 'verstecken, (sich) bedecken; sammeln, pflücken'

fi. korjata 'ernten, einsammeln; reparieren, korrigieren [...]' 
Laut SSA (I: 403) in einigen osfi. Sprachen belegt; osfi. (EES 178f.; EEW III: 955f.) liv: $1: 2,1: 19,1: 23$

estS: eS:20

30 est. kõr|s, gen. -re 'Halm' wot. kõrsi, gen. kõrrõõ id. estS. kõrs', gen. kõrrõ id.

fi. korsi 'Halm; Schlamm-Schachtelhalm'

? < balt. (SSA I: 406); < balt. (EES 202); osfi. (EEW IV: 1108)

est: e:3

wot: w:1

estS: eS:6, eS:9, eS:20

31 est. kõrv, gen. - a 'Ohr' wot. kõrva id. estS. kõrv, gen. kõrva id. | liv. kūora id.

fi. korva 'Ohr; Henkel'

Laut SSA (I: 408) in allen osfi. und einigen weiteren fiugr. Sprachen belegt; EES (202f.) bezeichnet den Stamm als osfi.-lp. oder ural. Gleichzeitig wird die Möglichkeit einer Entlehnung osfi.-lp. < indoeur. erwogen.; osfi.-lp. (?fiugr.) (EEW IV: 1110f.)

est: e:3

liv: $1: 2,1: 4$

estS: eS:6

32 wot. kõta 'Hausflur, Haus, Kammer' | est. koda, gen. koja id. liv. kuodā 'Haus, Kammer' estS. koda, gen. kua 'Hausflur, Haus, Kammer'

fi. kota 'kegelförmige Wohnung, Hütte, Zelt [...], Kote; Kochzelt' fiugr. *kota (SSA I: 411)

SSA (I: 411) erwägt eine mögliche Entlehnung von fiugr. *kota < indoeur. oder umgekehrt von fiugr. *kota > indoeur.; EES (168) erwägt ebenfalls einen fiugr. Stamm oder eine Entlehnung ? < indoeur.; fiugr. (EEW III: 880f.)

liv: $1: 2$

33 estS. kõ |tar', gen. -dara 'Speiche' [estN. kodar, gen. - $a$ id.] wot. kõtara id. liv. kõ'ddõrzid.

fi. ketara 'Schlittenstrebe; Bogen der Schlittenkufe; Bein'

< balt. (SSA I: 351; EES 168); fiugr. Wort (EEW III: 881)

est: Für das Estnische ist die estS. Variante als einschlägig anzusetzen. Laut UNIVERE (1986: 69) haben die estS. Dialekte den Laut - $\tilde{o}$ - bewahrt, während in estN. -o- vorliegt (vgl. auch KEEM 2002: 33). Ein Lautwandel $*_{-}-e_{-}>-\tilde{o}_{-}>-$- $o$ - ist für estN. wohl ansetzbar. 
Für eine derartige Entwicklung sprechen zudem die anderen Vertretungen, vgl. auch kar. ketara 'gebogenes Vorderteil der Schlittenkufe, Nacken' (SSA I: 351).

est: e:3

liv. 1:19, 1:41; $-z$ ist hier wohl analogisch (POSTI 1942: 277)

estS: eS:6, eS:20

34 est. kõva 'hart, fest, streng' wot. kõva id. liv. kõvā id. estS. kõva id.

fi. kova 'hart; fest; streng'

Laut SSA (I: 415) in allen osfi. Sprachen belegt; osfi. (EES 203); keine Herkunftsangaben in EEW (IV: 1113f.)

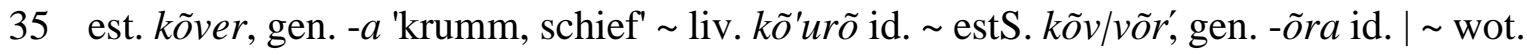
kovera id.

fi. kovera 'konkav, ausgehöhlt'

Laut SSA (I: 415f.) in allen osfi. Sprachen belegt; osfi. oder osfi.-md. (EES 203); osfi.(?-lp.) (EEW IV: 1115)

est: e:3

liv: $1: 19,1: 35,1: 40$

estS: eS:6, eS:9, eS:16, eS:20

36 est. krõmpsuda 'krachen, knacken' liv. krēmpš 'Kruste, Rösch, ein essbarer Pilz'

fi. ohne Entsprechung

Kein Eintrag in SSA; EES (185) diskutiert Ableitung von est. krompsuma 'krachen, knarren', was onomat. sei (ebd. 183).; MäGISTE (EEW III: 1005) erwägt für zugehöriges est. krõmps, $-u$ 'gewisser Ton, Laut' einen est.-liv. onomat. Stamm krõmps-.

37 est. lõbu 'Vergnügen' estS. lõbu id. | wot. Ø, vgl. jedoch wot. levätäg '(aus)ruhen lassen, rasten' zu fi. levätä (SSA II: 67f.) und est. lebada (EES 232)

fi. lepo 'Ruhe'

urfi. *lepo (LÄGLOS II: 193)

SSA (II: 64) verweist auf fi. levätä '(aus)ruhen, rasten', was in Verbindung zu est. lebada id. stehen soll. Dort wird est. lõbu nicht weiter behandelt. Bei fi. levätä wird eine Entlehnung erwogen, ? < germ., mhd. (SSA II: 67f.); EES (257) nennt für die Entwicklung von est. lõbu zwei Optionen: Es wird eine Ableitung von est. lebama 'ruhen, liegen' oder eine eigenständige Entlehnung erwogen, ? < urgerm.; vielleicht osfi. (EEW V: 1402); ? germ. Lehnwort oder Ableitung von levätä (LÄGLOS II: 193f.) est: e:2 
estS: eS:3

38 est. lõhe 'Lachs' wot. lõhi, gen. lõhõo id. estS. lôhekala; lôh|i, gen. -e id.

fi. lohi 'Lachs'

*losi- < balt. (EEW V: 1404)

< balt. (SSA II: 85; EES 258; EEW V: 1404)

39 est. lõhkeda 'bersten, platzen, sich spalten' wot. lõhgõta id. estS. lõhkõ-id.

fi. lohjeta 'sich (ab)spalten, zerfallen'

SSA (II: 86) erwägt ein vermutlich ursprünglich deskriptives Wort, welches den gleichen Ursprung wie fi. lohtua 'lösen, losmachen, trennen' haben könne.; EES (258) erwägt eine Variante vom Stamm est. lahk 'Scheitel, Schneise, Trennung'. Diese ist nicht gesichert.; MÄGISTE (EEW V:1408) setzt als Stamm osfi. *lohka( $\delta a)$ - an, von welchem sich auch est. lahkema entwickelt habe.

wot: w: 1

estS: eS:9

40 est. lõhmus 'Linde' estS. lõhmus id.

fi. lehmus 'Linde'

Möglicherweise deskriptiven Ursprungs (SSA II: 58); osfi., vielleicht onomat. (EES 258); osfi.-? wolg. (EEW V: 1405)

41 est. lõkatada 'aufflammen' | wot. lekko 'Flamme'

fi. lekko 'Flamme, Feuer; Hitze, Sonnenglut'

Laut SSA (II: 61) in einigen osfi. Sprachen belegt; In EES (258) wird ein onomat. Stamm angesetzt, der in keiner Verbindung zu anderen osfi. Wörtern stehen soll. Als „nahe Stämme“ werden est. rõkkama 'laut jauchzen, gellen, schallen' und est. logisema 'wackeln, klappern, klapprig sein' genannt, welche jedoch für den obenstehenden Beleg nicht einschlägig sind.; osfi. (EEW V: 1412)

42 est. lõng, gen. - a 'Garn, Faden' wot. lõyka id. | liv. lānga id. estS. lang, gen. langa id.

fi. lanka 'Faden, Garn; Draht'

urfi. *lanka (LÄGLOS II: 166)

< germ. (SSA II: 44); < urgerm. (EES 259); osfi-lp. < skand. (EEW V: 1417); germ. Lehnwort (LÄGLOS II: 166)

est: e:3

liv: $1: 1$ 
estS: eS:6

43 est. lóppeda 'beenden' wot. lõpõttaa id. estS. .lõpma, II. Infinitiv lõppõq id. | liv. loptõ id.

fi. lopettaa 'beenden'

SSA (II: 92) setzt den gleichen Urstamm wie für fi. loppu 'Ende' und fi. loppi 'Ecke, Winkel, Vertiefung' und Entsprechungen an.; osfi. Stamm (EES 259); MÄGISTE (EEW V: 1419f.) setzt einen osfi. Stamm *loppe- an.

wot: w:1

liv: $1: 19,1: 32$

estS: eS:7

44 est. mõdu 'Met' wot. mõtu 'Met, Bier' estS. mõdu 'Met'

fi. metu 'Honig, Saft'

urfi. *metu (LÄGLOS II: 261)

< ? balt. oder germ. (SSA II: 164; EES 291); MäGISTE (EEW V: 1589) erwägt eine balt. oder germ. Entlehnung oder eine Ableitung von [est. ?] mesi 'Honig'; ? germ. oder balt. Lehnwort (LÄGLOS II: 261f.)

45 liv. mõgā 'Geräusch, Dröhnen, Gedröhne' | est. möga 'Quatsch, Unsinn' wot. mökisä 'blöken, meckern; murmeln, [...]'

fi. mökä 'Geschrei, Lärm'

onomat. deskriptiv (SSA II: 196); osfi. onomat. (EES 297f.; EEW V: 1629)

est: e:1

liv: $1: 17$

46 est. mõjuda 'beeinflussen, wirken' estS. mõo|ma, II. Infinitiv -daq id. | liv. mojjjõ 'fühlen, wahrnehmen, wirken'

fi. mojoa 'schmerzen; wirken, helfen'

Laut SSA (II: 169f.) in den genannten osfi. Sprachen belegt; EES (291) setzt einen osfi. Stamm an und erwägt, dass es sich um den gleichen fiugr. Stamm wie bei est. mõista 'verstehen, begreifen' handeln könne.; MÄGISTE (EEW V: 1593) setzt eine osfi. Stamm *mojo an und betrachtet est. mõjuda als von est. mõju 'Wirkung, Ansehen' abgeleitet. est: e:12

liv: 1:19, 1:22

estS: eS:11 
47 est. mõksuda 'zusammenschlagen, zusammenstoßen [...]'

fi. moksahtaa 'klatschen, (hin)plumpsen'

onomat. (SSA II: 170); kein Eintrag in EES; osfi. onomat. (EEW V: 1593)

est: e:12; Wied. (SSA II: 170)

48 est. mõla 'Ruder' wot. mõla id. estS. mõla id.

fi. mela 'Ruder, Paddel'

Laut SSA (II: 158) in einigen osfi. und weiteren fiugr. Sprachen belegt; osfi-md. oder osfi.-perm. (EES 291f.); fiugr. (EEW V: 1594)

49 est. mõlema $(d)$ 'beide' wot. mõlõpad id. estS. mõlõmb|aq id. $\mid \sim$ liv. mó'lmõd id.

fi. molempi 'beide'

SSA (II: 170) setzt eine Ableitung vom Pronominalstamm *mo- an. Die Herkunft von -l- sei ungesichert.; In EES (292) wird erwogen, dass es sich um eine Stammvariante von est. тии 'andere/r/s' oder den gleichen Stamm wie bei est. mõni 'jemand' handeln könne.; MÄGISTE (EEW V: 1594) nennt als Ausgangsform ein osfi. Pronomen molen. wot: w:1

liv: $1: 19,1: 40$

wot: w:15

estS: eS:9

50 est. mõmiseda 'brummen' estS. mõmis| õma, II. Infinitiv -taq id.

? fi. mumista 'murmeln'

Keine Verbindung in SSA (II: 178); EES (292) bezeichnet den Stamm als osfi. onomat. und verweist auf weitere „nahe Stämme“, welche jedoch keine gesicherten Varianten sind.; MÄGISTE (EEW V: 1597) bezeichnet das Wort als est. onomat. und verbindet es nicht mit einer fi. Variante.

estS: eS:9

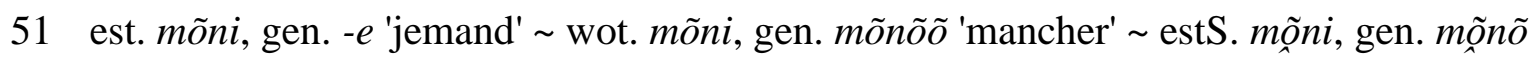
'jemand' | liv. mūnda 'mancher'

fi. moni 'mancher'

urfi. *moni < *mone (LÄGLOS II: 265)

SSA (II: 171) erwägt eine sehr alte indoeur. Verbindung, die auf eine mögliche Zusammengehörigkeit der Sprachen schließen könne. Diese sei nicht gegeben, wenn man von einer möglichen Ableitung der osfi. Wörter vom Pronominalstamm mo- mu- ausgehe. Bei der letzteren Entwicklung sei die Nähe zu indoeur. Formen Zufall.; EES (292) setzt 
eine unsichere alte Entlehnung an (indoeur.); fiugr. (EEW V: 1597f.); kein germ. Lehnwort (LÄGLOS II:265f.)

wot: w: 1

liv: 1:1; liv. Form = PartSg. (SSA II: 171, LW 237f.)

estS: eS:9

52 est. то̃пи 'Genuss [SSA auch: Lärm] estS. mõno 'Genuss' | wot. meno 'Gang' fi. meno 'Gehen, Gang, Fahrt, (Ver)lauf; Radau'

Die etymologischen Wörterbücher erwägen eine Ableitung von fi. mennä 'gehen' bzw. est. minema id. (SSA II: 160; EES 292), est. mõnu wird dort nicht weiter behandelt (SSA II: 159f.; EES 282); MÄGISTE (EEW V: 1599) setzt einen osfi.-lp. Stamm *meno 'Gehen, Gang' an.

est: e:2

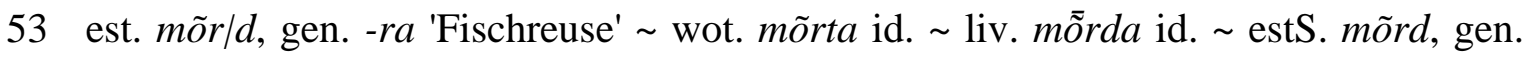
mõrra id.

fi. merta 'Fischreuse'

urfi. *merta (LÄGLOS II: 260f.)

< skand. (SSA II: 161; EEW V: 1600); urgerm. (EES 292); germ. Lehnwort (LÄGLOS II: 260f.)

est: e:3

liv. 1:1

estS: eS:6

54 est. mõrsja 'Braut' estS. .mõrsja id.

fi. morsian 'Braut'

< balt. (SSA II: 172; EES 292f.; EEW V: 1601)

55 est. mõtelda 'denken, nachdenken' $\sim$ wot. mõtõlla id. liv. mõtlõ id. $\sim$ estS. .mõtlõma, II. Infinitiv mõtõldaq id.

fi. ohne Entsprechung

urfi. *mettV- (LÄGLOS II: 281)

Eine Verbindung zu fi. miettiä 'nach-, bedenken, überlegen' sei laut SSA (II: 166f.) ungesichert. Ein russ. Lehnwort wird erwogen. Auch bei einer wenig wahrscheinlichen Verbindung zu est. mõtelda, welches < germ. sei, sei russ. Einfluss denkbar.; EES (293) nimmt eine Ableitung < urgerm. an. -le- sei ein osfi. Ableitungssuffix. MäGISTE (EEW 
V: 1603f.) setzt einen Stamm *metta- an, welcher germ. sei. Eine Zusammenstellung mit fi. miettiä lehnt er ab; germ. Lehnwort (LÄGLOS II: 281)

est: e:13

wot: w:1

liv: $1: 19,1: 23$

estS: eS:9, eS:21

56 liv. mõtsā 'Wald' $\sim$ estS. mõts, gen. mõtsa id. | est. mets, gen. $-a$ id. $\sim$ wot. mettsä id. fi. $m e t s \ddot{a}$ 'Wald'

? < balt. (SSA II: 163); < balt. oder fiugr. (EES 281); osfi.-lp. (EEW V: 1532f.)

est: e:1, e:3

liv: $1: 17$

estS: eS:6

57 est. mõtus, gen. -e (dial.) 'Auerhuhn, -hahn' liv. mõtūks id. estS. mõtus id.

fi. mettos 'Auerhuhn, -hahn'

Laut SSA (II: 164) in den genannten osfi. Sprachen belegt; osfi. oder fiugr. (EES 293);

? fiugr. (EEW V: 1604). In den etymologischen Wörterbüchern wird eine Verbindung zu fi. metso 'Auerhuhn, -hahn' bzw. est. metsis id. erwogen, welche eine volksetymologische Verbindung zu mets(ä) 'Wald' haben könne (vgl. SSA II: 163; EES 281)

est: e:2

liv: $1: 20$

estS: eS:3

58 est. nõbu 'Cousin'

fi. nepaa 'Cousin(e); Kind des Cousins/der Cousine'

< balt. (SSA II: 213; EES 323; EEW VI: 1756)

est: $-u$ sei laut EES (323) eine alte Ableitung

59 est. nõd|er, gen. -ra 'schwach, schwachköpfig' wot. nõdra id. liv. nó'ddõr id. estS. nõder, gen. nõdra id.

fi. nöyrä 'ergeben, unterwürfig, demütig'

*netra oder *neträ (EEW VI: 1756f.)

Laut SSA (II: 257) in einigen osfi. Sprachen belegt; osfi. (EES 323; EEW VI: 1756f.) liv. 1:19, 1:41

60 est. nõel, gen. - a 'Nadel' liv. nõ'ggõl id. estS. nõgõl; nõkl, gen. nõgla id. | wot. nigla id. 
fi. neula 'Nadel'

urfi. *nekla /*nēkla (LÄGLOS II: 297)

<germ. (SSA II: 215; EEW VI: 1758f.); < urgerm. (EES 323); germ. Lehnwort (LÄGLOS II: 297f.)

est: e:3, e:5

wot: w:5; ? kar. Einfluss (VKÄ 126)

liv: $1: 19,1: 41$

estS: eS:6

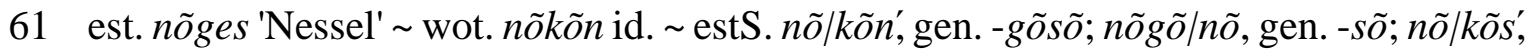
gen. $-g \tilde{o}(s) s \tilde{o} \mathrm{id}$.

fi. nokkonen 'Brennessel'

SSA (II: 229) erwägt eine Verbindung zu fi. nokka 'Schnabel', welche jedoch nicht gesichert ist; osfi. (EES 324; EEW VI: 1759f.)

wot: w:1

estS: eS:9, eS:20

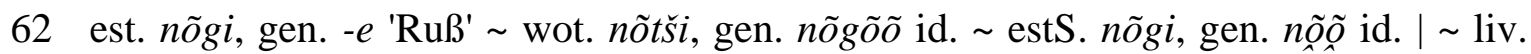
no'ugõd id.

fi. $n o k i$ 'Ruß'

Laut SSA (II: 228) in allen osfi. Sprachen belegt; osfi. (EES 324; EEW VI: 1760)

Wot: w:6

liv: $1: 3^{45}, 1: 19,1: 40$

estS: eS:9

63 est. nõ $\mid l g$, gen. -le 'Rotz' estS. nõlõ id. | liv. nolgõ 'geifern'

fi. nolki 'Schleim, Geifer'

urspr. deskriptives Wort (SSA II: 229f.); fiugr. (EES 324; EEW VI: 1762)

est: e:3

liv: $1: 19$

estS: eS:9

64 est. nõmm, gen. -e 'Heide' wot. nõmmi, gen. nõmmõ $\tilde{o}$ id. estS. nõmm gen. $-e$ id. | liv. num id.

fi. nummi 'Heide'

${ }^{45}$ Nach POSTI (1942: 49) ist wohl ansetzbar, dass es in der nichtersten Silbe zunächst eine Entwicklung $e>u$ gab. 65 
? osfi. *numi (SSA II: 238); osfi. oder ural. (EES 325); osfi. (EEW VI: 1763)

est: e:3

estS: eS:6

65 est. $n \tilde{o r} \mid k$, gen. - ga 'schwach' wot. nõrkka 'düster, finster, missmutig, wehmütig' liv. nõ $r k a$ 'schwach' estS. nõrk, gen. nõrga id.

fi. nerko 'klein, schwach, zart'

Laut SSA (II: 214) keine Zusammenstellung der Belege mit der wotischen Entsprechung; EES (325) setzt einen osfi. oder osfi.-perm. Stamm an und nennt als wot. Form nõrkk; osfi. (? fiugr.) (EEW VI: 1766f.)

est: e:3

liv. 1:1

estS: eS:6

66 est. nõtke 'biegsam, geschmeidig, weich' wot. nõtkõa id. liv. nõtkõ 'biegen, beugen, krümmen' estS. .nõtskõ 'biegsam, geschmeidig, weich'

fi. notkea 'biegsam, geschmeidig; weich'

Laut SSA (II: 233f.) in allen osfi. Sprachen und im Lp. belegt; EES (325) setzt für das zugehörige est. nõtk, gen. - $u$ 'Biegung; Senkung, Niederung, niedrig gelegenes Land' als Ausgangsform einen osfi.-md. Stamm an.; MÄGISTE (EEW VI: 1769f.) setzt für eine est. $n \tilde{o} t k$, gen. $-u$ einen urosfi.-lp. Stamm an.

Als Stamm ist *notke- $\delta a$ anzusetzen. Zum Ableitungssuffix - $\delta A$ und seinen einzelsprachlichen Vertretungen s. LAANEST (1982: 205).

est. e:3, e:4

wot. w:1, w:10

liv. 1:19, $1: 23$

estS: eS:9, eS:10

67 liv. $\tilde{o}^{\prime} b b i$ 'Pferd' | est. hobune id. wot. opõnõ id. estS. ho|põn', gen. -bõsõ

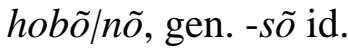

fi. hevonen hepo 'Pferd'

urfi. *hepo (LÄGLOS I: 95)

Laut SSA (I: 156, 160) in allen osfi. Sprachen belegt; EES (77) setzt einen osfi. Stamm an und erwägt eine mögliche germ. Entlehnung, für welche jedoch keine Quellen gefunden wurden.; osfi., vielleicht < hepoi (EEW II: 366f.); kaum germ. Lehnwort (LÄGLOS I: 95f.) 
est: e:2

wot: w:1, w:11; ? estnischer Einfluss (VKÄ 126)

liv: 1:27, 1:38, 1:39, 1:40; Bei - $i$ im Auslaut setzt KeTtUnen (LW XXXIX) die Vertretung eines alten Diphthongs an $(<*$ hevoinen $)($ ebd. XXVIII)

estS: eS:6, eS:9, eS:20

68 wot. oggaz 'Dorn, Stachel' | est. ok|as, gen. -ka id. liv. ? oga-pä̈ 'Starrkopf, eigensinnig' estS. oha|k, gen. - gu 'Dorn, Stachel'

fi. oas 'Dorn, Stachel, Widerhaken; Granne'

Unbekannte Herkunft (SSA II: 258); EES (336) setzt est. okas als Ableitung von est. oga 'Dorn, Stachel' an, was osfi. sei (ebd. 333f.); MÄGISTE (EEW VI: 1824f.) geht von einem osfi. Stamm aus und setzt für diesen *okaha- an.

liv: möglicherweise deskriptiv (POSTI 1942: 9)

estS: ? $k>h(v g l$. EKÄ 57)

69 est. õhata 'seufzen; ausströmen' estS. .õhkama, II. Infinitiv õhadaq id.

fi. hohkua 'Wärme oder Kälte ausstrahlen'

onomat. deskriptiv (SSA I: 169); osfi. onomat. (EES 625); kein Eintrag in EEW

70 est. $\tilde{o} h k$, gen. $\tilde{o} h u$ 'Luft' liv. $e^{\prime} g \partial$ 'glühen, schmerzen' estS. (h)õhk, gen. (h)ôhu 'Luft'

fi. hehkua 'glühen'

*hehku (EEW XII: 4011)

Laut SSA (I: 150) in einigen osfi. Sprachen belegt; EES (624) setzt einen osfi. onomat.

Stamm an, welcher sich möglicherweise mit einem Stamm õhkama vermischt habe.;

osfi. onomat. (EEW XII: 4011)

est: e:3

liv: 1:19, 1:33

estS: eS:6

71 wot. ôhja 'Leine, Zügel' | est. oh|i, gen. ja id. liv. ü'olmõz id. estS. uhi, gen. uhá $\sim$ ohi, gen. ohá id.

fi. ohja 'Zügel'

urfi. *ohja / *ohjas (LÄGLOS II:309)

? < (ur)germ. (SSA II: 259; EES 334); osfi. (EEW VI: 1817f.); ? germ. Lehnwort (LÄGLOS II:309)

liv: 1:4, 1:19, 1:33

estS: eS:1, eS:20 
72 wot. õhsa 'Ast, Zweig' | est. oks, gen. - a id. liv. oksā id. estS. ossa, gen. ossa id. fi. oksa 'Zweig, Ast'

Laut SSA (II: 262) in allen osfi. und einigen weiteren fiugr. Sprachen belegt; osfi.tscher. oder osfi.-perm. (EES 336); fiugr. (EEW VI: 1826)

est: e:3

wot: w:8

estS: eS:14

73 wot. õhsõta 'sich übergeben' | est. oksendada id. liv. oksnõ id. estS. ossõnda|ma, II. Infinitiv $-q$ id.

fi. oksentaa '(sich) erbrechen'

Laut SSA (II: 262) in allen osfi. und einigen weiteren fiugr. Sprachen belegt; osfi.perm. (EES 336); keine Herkunftsangaben in EEW (VI: 1826f.)

wot: w1, w:8, w:14

liv: $1: 19,1: 22$

estS: eS:9, eS:14

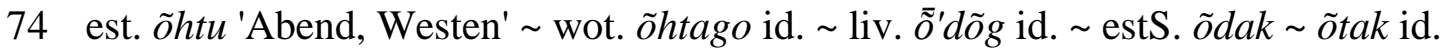

fi. ehtoo 'Abend; Abendessen'

Laut SSA (I: 101) in einigen osfi. Sprachen belegt; osfi. (EES 625); MÄGISTE (EEW XII: 4013) nimmt urosfi. *ekta; an. Die osfi. Formen seien von *ehta, *ekta mit Suffix $*_{-} \gamma o\left(?^{*}-\gamma a\right.$ ?) gebildet.

est: e:2

wot: EES (625) setzt alte Ableitung an

liv: 1:19, 1:21, 1:33; EES (625) setzt alte Ableitung an

estS: eS:6; EES (625) setzt alte Ableitung an

75 est. ôhuke 'dünn' wot. ôhuD id. $\mid \sim$ estS. .oh $(k u) k \tilde{o}|n \tilde{o} \sim . o h k u g \tilde{o}| n \tilde{o} \sim o h u k \tilde{o} \mid n \tilde{o}$, gen. -sõ id.

fi. ohut 'dünn'

SSA (III: 260) erwägt, dass das Beispiel auf den gleichen Stamm wie fi. ohimo 'Schläfe; Leiste' zurückgeht. Dieser sei *oha- (ebd. 259); osfi. (EES 625); osfi., von urosfi. eha (EEW XII: 4009)

est: e:14

wot: w:21

estS: eS:9; Verbreitung von $h k$ wird von KEEM (2002: 30) nicht genauer eingegrenzt 
76 wot. õja 'Bach' | est. oja id. liv. v(u)ojā, vojjā 'mit Wasser gefüllte Vertiefung, Pfütze' estS. oja; uja 'Bach'

fi. oja 'Graben; Bach'

Laut SSA (II: 262) in allen osfi. Sprachen und im Lp. belegt; EES (335f.) erwägt die Ableitung eines alten Stammes, zu dem auch est. voog 'Woge, Welle' gehören soll. Die Ableitung ist nicht gesichert.; MÄGISTE (EEW VI: 1823f.) erwägt eine mögliche osfi. Herkunft und setzt einen Stamm *oja- an.

liv: $1: 2,1: 5$

77 liv. ó 'ldzõ 'hell, grell, klar' | est. hele 'hell, klar' wot. eliä id. estS. helle id. fi. heleä 'hell, klar'

Laut SSA (I: 152) in allen osfi. Sprachen belegt; EES (73) setzt einen osfi. onomat. Stamm an, welcher möglicherweise in est. helge 'hell, freundlich, schwül' und est. helkima 'schimmern, glänzen' zu finden sei. Die Verbindung ist nicht gesichert.; MÄGISTE (EEW I: 323) setzt einen osfi. onomat. Stamm *helä- an.

Als Stamm ist *hele- $\delta A$ anzusetzen. Zum Ableitungssuffix $-\delta A$ und seinen einzelsprachlichen Vertretungen s. LAANEST (1982: 205)

est: e:3, e:4

wot. w:1; w:10, w:11

liv: 1:19, 1:23, 1:27, 1:40; KETTUNEN (LW 51) setzt für liv. <*heleda- + -nen : -sen an. estS: eS:6, eS:9, eS:16

78 est. $\tilde{o} l \mid g$, gen. - $a$ 'Schulter' | estS. olg, gen. ola id.

fi. olka 'Schulter'

Laut SSA (II: 263) in allen osfi. und einigen weiteren fiugr. Sprachen belegt; fiugr. (EES 626; EEW XII: 4022)

est: e:3

estS: eS:6

79 est. $\tilde{o l} \mid g$, gen. -e 'Stroh, Halm' wot. õltši, gen. ólgõ $\tilde{o}$ id. $\mid \sim$ liv. vólg id. $\sim$ estS. olg', gen. olõ id.

fi. olki 'Stroh, Halm'

Laut SSA (II: 263f.) in allen osfi. Sprachen und im MdE. belegt; osfi.-md. (EES 626); osfi.-lp.-md. (EEW XII: 4023)

est: e:3

wot: w:1, w:6 
estS: eS:6, eS:9, eS.24

80 wot. ôlla 'sein' | est. olla, olen-1SGPRÄSAKT id. liv. vōlda id. estS. olõma, II. Infinitiv ollaq id.

fi. olla 'sein'

ole- (< fiugr. *vole-), von Stamm o- (SSA II: 264)

SSA (II: 264) erwägt eine mögliche, ursprünglich frequentative Ableitung *ole- vom Stamm ${ }^{*} o-$, von welchem auch fi. oma 'eigen' abgeleitet sei und welcher die Grundlage für die Form on 'sein-3SGPRÄSAKT' bilde. EES (336) geht ebenso von einem fiugr. Stamm aus, von dem est. oma 'eigen' abgeleitet sein könne; MÄGISTE (EEW VI: 1828) setzt einen fiugr. Stamm an und vermutet, dass est. olla aus einer Form *oldak entstanden sei.

liv: $1: 5$

estS: eS:9

81 est. $\tilde{o} l \mid u$, gen. $-l e$ 'Bier' $\sim$ wot. õlu| $\mid$, gen. $-u$ id. $\mid \sim$ liv. $v \dot{o}^{\prime} l$ id. $\sim$ estS. oluq, gen. ollõ id. fi. olut 'Bier' urfi. *olut (LÄGLOS II: 310)

< balt. oder germ. (SSA II: 264f.; EEW XII: 4025); Auch EES (626) erwägt eine balt. oder germ. Entlehnung, germ. sei aus lautlichen Gründen unsicher; ? germ. oder balt. Lehnwort (LÄGLOS II: 310)

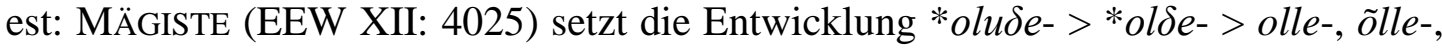
elle- mit „ungewöhnl. Synkope“ an.

liv: 1:5, 1:40

estS: eS:9

82 wot. $\tilde{\text { oma }}$ 'eigen' | est. oma id. liv. $u^{\prime} m$ id. ${ }^{46} \sim$ estS. uma id.

fi. oma 'eigen'

SSA (II: 265) erwägt eine Ableitung vom Stamm $o$ - mit dem Suffix -ma.; EES (337) erwägt eine Ableitung eines alten Stammes, von welchem auch est. olema abstamme, ebenso mit Suffix -ma (EES 337); fiugr. (EEW VI: 1831ff.)

liv: $1: 6,1: 40$

estS: eS:2

${ }^{46}$ Die Form liv. $u$ 'm 'eigen' wird durch SSA (II: 265) und KETTUNEN (LW 451) gestützt. LELS (343) übersetzt diese Form mit 'ich bin, er/sie/es ist'. Als livische Form von 'eigen' wird dort stattdessen $u$ 'mmi genannt (ebd. 344). 
83 est. ómmelda 'nähen' wot. ómmõlla id. | liv. umblõ id. estS. .umblõma id.

fi. ommella 'nähen'

Laut SSA (II: 266) in allen osfi. Sprachen und unsicher im Lp. belegt. Die Formen mit $u$ - im Anlaut seien sekundär. Ein Einfluss von fi. umpi 'ge-, verschlossener, verstopfter Zustand' wird erwogen.; osfi. oder osfi.-lp. (EES 626; EEW XII: 4025)

wot: w:1

liv: 1:6, 1:23, 1:46

estS: eS:2, eS:9

84 est. õng, gen. -e 'Angel(haken)' wot. ontši, gen. õndžõ õ id. estS. õng', gen. õngõ id. fi. onki '(Angel)haken'

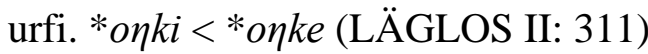

? < indoeur., urgerm. (SSA II: 267); < indoeur. (EES 626); ? < ar. (EEW XII:4026f.);

? germ. Lehnwort (LÄGLOS II: 311)

est: e:3

wot: w:1, w:6

estS: eS:6, eS:9, eS:20

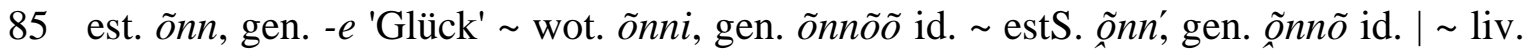
von id.

fi. onni 'Glück'

SSA (II: 267f.) erwägt eine Entstehung von fi. onni, gen. onne- durch die Bildung falscher Nominalformen aus den Flexionsformen des Wortes fi. onsi, gen. onte-. Für die liv. Entsprechung sei in diesem Fall anzusetzen, dass sie aus einem anderen Dialekt entlehnt sei, welcher -nn- als schwache Stufe von -nt- habe.; EES (626f.) erwägt eine Variante von est. õõs 'Höhle, Höhlung' oder ein vorgerm. Lehnwort; MÄGISTE (EEW 4028-4031) bezeichnet den Stamm als osfi.-lp. und erwägt eine mögliche frühurfi.-lp. Ableitung von fiugr. *ońća (< ar.); kein Eintrag in LÄGLOS

est: e:3

wot: w:1

liv: $1: 5$

estS: eS:6, eS:9

86 est. $\tilde{o} \tilde{o} \mid s$, gen. -ne 'Höhle, Höhlung' wot. $\tilde{o} \tilde{o s i}$, gen. $\tilde{o} \tilde{o} \tilde{\tilde{o}} \tilde{o} \mathrm{id} . \sim \operatorname{liv} . \overline{\tilde{o}} n \mathrm{id}$.

fi. onsi 'hohle Stelle, Höhlung; hohl, leer' 
In SSA (II: 268) werden zwei mögliche Urstämme angesetzt, *omte oder *oฑte; EES (628) geht von osfi.-lp. oder fiugr. Formen aus. Es wird eine Verschmelzung zweier fiugr. Stämme im osfi. erwogen; MÄGISTE (EEW XII: 4040) setzt fiugr. *omte (? *oฑte) und eine Entwicklung est. < urosfi. onsi an.

est: e:3, e:8

wot: w:10, w:13

liv: ? < est. (SSA II: 268); EES (628) setzt eine sichere est. Entlehnung an.

87 est. oppida '(er)lernen' wot. õppõa id. | liv. oppõ id. estS. .oṕma, II. Infinitiv oppiq id.

fi. oppia '(er)lernen'

Laut SSA (II: 269f.) in allen osfi. Sprachen und im Obugr. belegt; fiugr. (EES 627; EEW XII: 4031-4034)

wot: w:1

liv: 1:19, 1:23

estS: eS:7, eS:20

88 wot. ora 'Ahle, Pfriem, Metallstift, scharfe Spitze' | est. ora id. liv. vorrā 'Spitze' estS. ora 'Ahle, Pfriem, Metallstift, scharfe Spitze'

fi. ora 'Stachel, Dorn; Bohrer [...]'

? < urar. oder uriran. (SSA: II: 270); < urindoiran. (EES 338); fiugr. < ? frühindoiran. (EEW VI: 1840)

liv: 1:5

89 wot. orrava 'Eichhörnchen' | est. orav, gen. - $a$ id. liv. vörābõz/v(u)orābaz id. estS. or|rav, gen. -ava id.

fi. orava 'Eichhörnchen'

Laut SSA (II: 270f.) in allen osfi. und einigen weiteren fiugr. Sprachen belegt; osfi.perm. (EES 338); osfi.-lp. (EEW VI: 1841)

est: e:3

liv: $1: 2,1: 5,1: 19$

estS: eS:6, eS:16

90 est. $\tilde{o r} \mid s$, gen. -re 'Stange, Hühnerstange' wot. õrsi, gen. õrrõõ id. | liv. vórž 'Wipfel, Stange' estS. ors', gen. orrõ 'Stange, Hühnerstange'

fi. orsi 'Balken; Hühnerstange'

< balt. (SSA II: 272; EES 627; EEW XII: 4035f.) 
est: e:3

wot: w:10

liv: $1: 5,1: 30$

estS: eS:6, eS:9, eS:20

91 wot. $\tilde{o s a}$ 'Teil' | est. osa id. liv. vózā id. estS. osa id.

fi. $o s a$ 'Teil, Anteil'

? < urar. (frühuriran.) (SSA II: 273); ? < urindoran. (EES 339); ohne Herkunftsangabe in EEW (VI: 1847ff.)

liv: $1: 5$

92 wot. óssaa 'kaufen' | est. osta id. liv. vóstõ id. estS. .ostma, II. Infinitiv .ostaq id. fi. ostaa 'kaufen'

wahrscheinlich < indoeur. (SSA II: 274; EES 340); fiugr. (EEW VI: 1853f.)

liv: 1:5, 1:19

93 wot. ottsa 'Kopf, Ende' | est. ots, gen. - a 'Ende, Kopf, Spitze' liv. vöntsa/vūontsa 'Stirn, hervorstehender oberer Teil der Stirnseite von etw.' estS. ots, gen. otsa 'Kopf, Ende'

fi. otsa 'Stirn, hervorstehender oberer Teil der Stirnseite von etw.' urfi. *o(n)cca < ońćća (LÄGLOS II: 312)

? < (ur)germ. (SSA II: 275f.; EES 340); fiugr. (EEW IV: 1854ff.); ? germ. Lehnwort (LÄGLOS II: 312)

est: e:3

wot: w:9

liv: $1: 2,1: 5$

estS: eS:6

94 wot. ôttsia 'suchen' | est. otsida id. liv. vötšõ id. estS. .otśma, II. Infinitiv .otsiq id. fi. etsiä 'suchen' urfi. *ecci- / *occi- (LÄGLOS I: 58)

SSA (I: 109) bezeichnet die Überlegung als unklar, ob das osfi. Wort (sposfi *ettsi-) auf fosfi. *eńćći-, *eńćće- oder *eńććä- + j- zurückgehe, welches < frühgerm. sei; ?< urgerm. (EES 340); osfi.-lp.-md. (EEW VI: 1857); ? germ. Lehnwort (LÄGLOS I: 57f.) wot: w:9

liv: 1:5, 1:19; analoge Bildung zu 1:22

estS: eS:7, eS:20 
95 wot. õzra 'Gerste' | est. oder, gen. odra id. liv. vó'ddõrz/v(u)o'droz id.

fi. ohra 'Gerste'

< balt. (SSA II: 259); ? > balt. (EES 333); MÄGISTE (EEW VI: 1813) setzt einen osfi.

Stamm an und hält eine indoeur. Entlehnung für unwahrscheinlich.

liv: 1:5, 1:19, 1:44

96 wot. õzża 'Schachtelhalm' | est. osi, gen. osja id. liv. vöžā id. estS. osi, gen. os'a id.

fi. hosia '(Winter-, Schlamm-) Schachtelhalm'

SSA (I: 174) erwägt den gleichen möglichen Ursprung wie für fi. huosia 'Schrubber oder Bürste aus Birkenrinde, Reisig o.ä.', welcher jedoch nicht benannt wird (ebd. 188);; osfi. (EES 339); MÄGISTE (EEW VI: 1849f.) setzt einen osfi.(-?fiugr.) Stamm und eine osfi. Form *hosja an.

wot: w:16

liv: 1:5, 1:31

estS: eS:20

97 est. põdeda 'leiden, krank sein' estS. põ |dõma, II. Infinitiv -tõq id. | liv. pó'ddõ 'schmerzen'

fi. potea 'leiden, krank sein'

Laut SSA (II: 405) in einigen osfi. Sprachen und im Lp. belegt; osfi.-lp. (EES 398; EEW VII: 2266)

liv: 1:19, 1:40

estS: eS:9

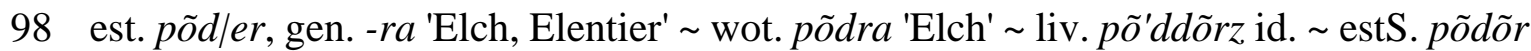
$\sim$ põtr, gen. põdra 'Elch, Elentier'

fi. peura 'wildes Rentier; Waldrentier, Elch'

SSA (II: 346) und EES (398) erwägen eine mögliche Entlehnung aus dem Baltischen; MÄGISTE (EEW VII: 2267) nimmt < osfi. *petra an und lehnt eine baltische Lehnetymologie ab.

liv: 1:19, 1:41; - $z$ ist hier wohl analogisch (POSTI 1942: 277)

estS: eS:9

99 est. põh $\mid i$, gen. -ja 'Boden, Grund' wot. põhja id. estS. põhi, gen. põhá id. | liv. pū'oj id.

fi. pohja 'Boden, Grund; Norden' 
urfi. *pohja (LÄGLOS III: 70)

SSA (II: 383) setzt zwei Möglichkeiten an: autochthone Entsprechungen oder ? < frühgerm.; ? < urgerm. oder ? < urindoiran. (EES 398); osfi.-lp. (EEW VII: 2269ff.)

Kein germ. Lehnwort, es wird eine indoiran. Entlehnung erwogen (LÄGLOS III:70f.). liv: $1: 2,1: 4,1: 33$

estS: eS:20

100 est. põhk, gen. $-u$ 'Kurzstroh, Streu' $\sim$ wot. põhku id. $\sim$ liv. $p \overline{\tilde{o}} ' k t$ 'feiner Staub' $\sim$ estS. põhk, gen. põhu 'Kurzstroh, Streu'

fi. pehku 'Stroh nach dem Dreschen, Spreu, Streu; (trockener Holz)abfall'

SSA (II: 328) bezeichnet die Beispiele als deskriptiv und geht von einem gleichen Ursprung wie fi. pehko 'Busch, Strauch' und fi. pehu 'zerbröseltes, morsches Holz, Streu; Abfall, Spreu' aus.; osfi. (EES 398; EEW VII: 2271f.)

est: e:3

liv: 1:21, 1:33 bzw. 1:29; $-t$ ist offensichtlich pluralisch (LW: 284)

estS: eS:6

101 est. põlata 'verachten' wot. põlgõt/taa 'mit Pferden tretend das Korn dreschen' estS. .põlgma, II. Infinitiv .põlgõ q 'verachten' | liv. pólgõ 'schmähen, lästern, beschuldigen, tadeln, verachten'

fi. polkea 'treten'

Laut SSA (II: 390) in allen osfi. Sprachen belegt; osfi. (EES 399); urosfi. Stamm *polke- (EEW VII: 2280ff.)

wot: w:1; pelgẹttās ? < est. (SSA II: 390)

liv: 1:19, 1:23

estS: eS:7, eS:9

102 est. põl|d, gen. -lu 'Feld' wot. põlto 'Feld, Ackerstreifen' estS. põld, gen. põllu 'Feld' fi. pelto 'Acker, Feld' urfi. *pelto (LÄGLOS III: 48f.)

< germ. oder schon vorgerm. (SSA II: 334); < urgerm. oder älter (EES 399); < germ. (EEW VII: 2278); germ. oder älteres Lehnwort (LÄGLOS III: 48f.)

est: e:2, e:3

estS: eS:6

103 est. põleda 'brennen' wot. põlõa id. | liv. pa'llõ 'Heu wenden; brennen' estS. pal|ama, II. Infinitiv -laq 'brennen' 
fi. palaa 'brennen'

SSA (II: 298) erwägt fi.-wolg. *pala- 'brennen'. Dieser Stamm gehöre möglicherweise zu ural. *pala- 'schlucken; Happen'; EES (399) setzt einen osfi.-md. Stamm an und diskutiert, dass es sich um den gleichen Stamm wie bei est. pala 'Stück, Biss, Happen' handeln könne. Die dort genannten Entsprechungen sind in diesem Rahmen nicht einschlägig (ebd. 349); MÄGISTE (EEW VII: 2279) nimmt ein fiugr. Wort an, das eine Variante zu est. palama 'brennen' sei.

wot: w:1

liv: 1:19, 1:40

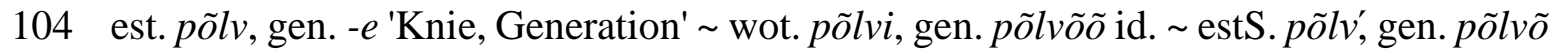
id. | liv. pūola id.

fi. polvi 'Knie; Generation'

Laut SSA (II: 392f.) in allen osfi. und weiteren fiugr. Sprachen belegt; ural. (EES 400; EEW VII: 2283f.)

est: e:3

wot: w:1

liv: 1:4, 1:36, vgl. dort: pùola : (PARTSG.) pol̀lâ

estS: eS:6, eS:9

105 liv. põnktõ 'strammen' | est. pingutada 'sich anstrengen, anspannen' estS. pingu|tama, II. Infinitiv -taq id.

fi. pingottaa '(an-, auf)spannen'

Laut SSA (II: 368) in einigen osfi. Sprachen und im Lp. belegt; EES (371) nimmt est. pinge 'Spannung, Anstrengung' als Ausgangsform an, welche osfi.-lp. oder osfi-tscher. sei.; MÄGISTE (EEW VII: 2064f.) erwägt est. ping, gen. - $u$ '[in Zuss.] gespannt, Spannungs-' als Ausgangsform, welche osfi. und vielleicht deskriptiv sei.

liv: 1:19, 1:22

106 est. põõsas 'Busch' wot. põõzaz id. liv. põ̃zõ id.

fi. pensas 'Busch'

Laut SSA (II: 337) in allen osfi. Sprachen und im Lp. belegt; osfi.-lp. (EES 402); MÄGISTE (EEW VII: 2301f.) setzt urosfi. pensas (? pensas) an.

est: e:8

wot: w:13

liv: 1:19. 1:34 


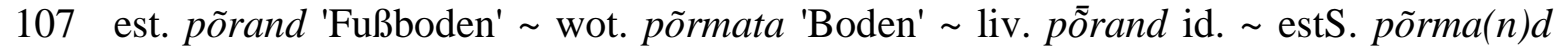
'Fußboden'

fi. permanto 'Fußboden; Dielenbrett'

SSA (II: 340) erwägt, dass es sich bei fi. permanto um eine Ableitung vom selben Stamm handelt wie est. põrm 'Staub, Asche'; In EES (400) wird der Stamm als osfi.-lp. bezeichnet und eine Ableitung von est. põrm als unsicher; osfi.-md. (EEW 2288) est: e:3; e:9

wot: w:14

liv: $1: 1$

108 est. põrm, gen. $-u$ 'Staub, Asche' $\sim$ wot. põrmu id. liv. põrm id. $\sim$ estS. põrm, gen. $-u$ 'Staub, Asche [...]'

fi. ohne Entsprechung

Kein Eintrag in SSA; < balt. (EES 401); est.-liv. (EEW VII: 2292)

est: e:3

liv: $1: 21$

estS: eS:6

109 est. põrn, gen. - $a$ 'Milz' wot. põrna id. liv. põ̃rna id. estS. põrn, gen. põrna id. fi. perna 'Milz'

Laut SSA (II: 341) in allen osfi. Sprachen belegt; ? < indoiran. (EES 401); osfi. (EEW VII: 2293)

est: e:3

liv: 1:1

estS: eS:6

110 est. põrsas 'Ferkel' wot. põrzaz id. estS. põrss, gen. .põrsa id. | liv. pūoraz id.

fi. porsas 'Ferkel'

< vorar. (SSA II: 400f.); < urindoiran. oder indoeur. (EES 401); MÄGISTE (EEW VII: 2294f.) setzt eine vorindoir. Entlehnung an, aus der ein Stamm urfiugr. *porśas (? *portśas) entstanden sei.

liv: $1: 4$

estS: eS:6, eS:13

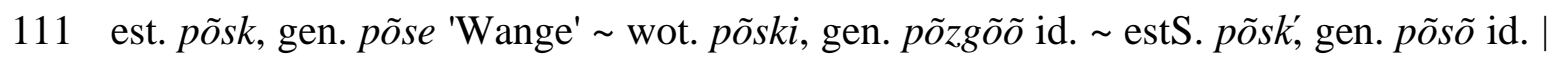

$\sim$ liv. posk id.

fi. poski 'Wange' 
Laut SSA (II: 403) in einigen osfi. und weiteren fiugr. Sprachen belegt; ural. (EES 401f.; EEW VII: 2296). MÄGISTE (EEW VII: 2296) erwägt zudem eine Entlehnung des wot. Beispiels aus dem Estnischen.

est: e:3

liv: $1: 21$

estS: eS:6, eS:9, eS:20

112 est. põtk, gen. - a 'Bein, Schenkel' wot. põtkõa 'treten' | liv. pótkāstõ id.

fi. potka 'Hinterfuß (Rind) bzw. -bein (Schwein) eines Schlachttieres'

Laut SSA (II: 405) in einigen osfi. und weiteren fiugr. Sprachen belegt; EES (402) bezieht sich auf die Variante est. põtk, gen. - $u$ 'Schenkel', welche osfi.-lp. oder ural. sei; osfi.-lp. (EEW VII: 2298);

est: e:3

liv: $1: 19$

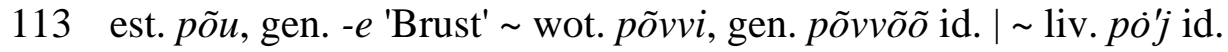

fi. povi 'Busen, Brust'

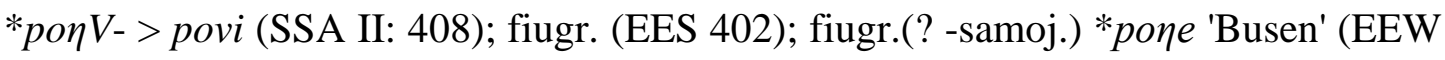
VII: 2299)

est: e:3, e:10

wot: w:1, w:17

liv: 1:40; $i=j$ (vgl. Transkription po ' $i$ (LW 309)).

114 wot. rõhgaa 'sehr, sehr viel, ziemlich' | est. rohke 'reichlich' estS. .rohkõ id.

fi. rohkea 'mutig, kühn; (als Mengenangabe) viel, reichlich'

urfi. *rohke- $\delta a$ (LÄGLOS III: 167)

Zum Ableitungssuffix $-\delta A$ und seinen einzelsprachlichen Vertretungen s. LAANEST (1982: 205)

Für fi. rohkea setzt SSA (III: 86) an: ? < altnord. oder gleicher Ursprung wie fi. rohtia 'Mut haben, wagen' oder fi. rohka 'porös, spröde; mürbe [...]'; ? < urgerm. (EES 433); osfi. (EEW VIII: 2515f.); germ. Lehnwort (LÄGLOS III: 167)

est: e:3, e:4

wot. w: $1, \mathrm{w}: 10$

estS: eS:6, eS:9

115 est. rõht, gen. rõ hu 'waagerecht seiend (Holz in der Wand, Stange im Zaun)' liv. rõ'ut 'Stangenlänge zwischen zwei Zaunpfosten' 
fi. rehto 'Abstand zwischen zwei Pfählen im Zaun oder Heureiter' urfi. *rehto (LÄGLOS III: 142)

< (ur)germ. (SSA III: 60; EES 441); wohl < germ. (skand.) (EEW VIII: 2571); germ. Lehnwort (LÄGLOS III: 142f.)

est: e:2

liv: ? < est. (LÄGLOS III: 142f.; EEW VIII: 2572; LW 335); POSTI (1942: 246) setzt

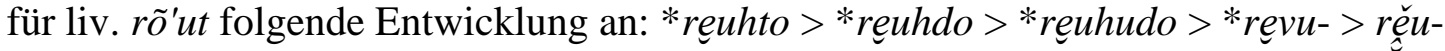

116 est. rõkkata 'laut jauchzen, gellen, schallen' liv. rõkkõ 'sagen, ansprechen' estS. rõkkama 'laut jauchzen, gellen, schallen'

fi. ohne Entsprechung

Kein Eintrag in SSA; onomat. (EES 441); est.-liv. onomatopoetisch (EEW VIII: 2574) liv: $1: 19$

117 est. rõngas 'Ring, Reifen' wot. rõygaz id. estS. rõngas, gen. .rõng

fi. rengas 'Ring, Reifen'

urfi. *reygas (LÄGLOS III: 145)

< urgerm. (SSA III: 64; EES 442; EEW VIII: 2575); germ. Lehnwort (LÄGLOS III: 145)

118 liv. rõzā 'Reuse' | est. rüsa 'Fischreuse' wot. rüsä id.

fi. rysä 'Fischreuse'

urfi. *rüsä (LÄGLOS III: 199)

< schwed. oder bereits urgerm. (SSA III: 119; EES 451); osfi. < skand. (EEW VIII: 2632); jüngeres Lehnwort (LÄGLOS III: 199)

est: e:1

liv: ? < est. (SSA III: 119; EES 451; EEW VIII: 2632; LÄGLOS III: 199)

119 est. sõba 'Decke, Bettdecke, wollenes Umschlagetuch' wot. sõpa 'Bekleidung, Kleider' estS. sõba 'Decke, Bettdecke, wollenes Umschlagetuch'

fi. sopa 'Bekleidung, Kleider'

Laut SSA (III: 200) in allen osfi. Sprachen und im Wog. belegt; fiugr. (EES 492); osfi.wog. (EEW IX: 2945f.)

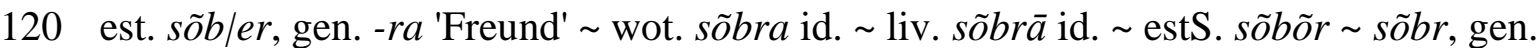
sõbra id.

fi. seura 'Gesellschaft, Zusammensein, Kreis von Menschen' ? < balt. (SSA III: 172; EES 492; EEW IX: 2946f.) 
liv: < est. (SSA III: 172; EES 492)

estS: eS:9

121 est. $s \tilde{o} \mid d a$, gen. -ja 'Krieg' $\sim$ wot. sõta, gen. sõaa id. estS. sõda, gen. sõa id. | liv. suodā id.

fi. sota 'Krieg'

Laut SSA (III: 204) in einigen osfi. Sprachen belegt; osfi. oder osfi.-wolg. (EES 493); osfi.-?lp.-md.-tscher. (EEW IX: 2947)

liv: $1: 2$

122 est. sõel, gen. - $a$ 'Sieb' liv. sõ'ggõl id. estS. sõgõl; sõkl, gen. sõgla id. | wot. sigla id.

fi. seula 'Sieb'

urfi. *sekla / *sēkla (LÄGLOS III: 229)

< (ur)germ. (SSA III: 171; EES 493); < germ. (EEW IX: 2948f.); germ. Lehnwort (LÄGLOS III: 229)

est: e:3, e:5

wot: w:5; ? kar. Einfluss (VKÄ 126)

liv: $1: 19,1: 41$

estS: eS:6, eS:9

123 est. sõge 'blind' wot. sõkõa id. estS. sõkõ 'dunkel, blind' | liv. so'gdə 'blind'

fi. sokea 'blind'

Die etymologischen Wörterbücher erwägen ungesicherte Ableitungen, vgl. fi. soka 'Schmutz, Müll, Unrat [...]' und est. soga 'Trübes, Bodensatz, Schlamm' (SSA III: 194; EES 493; EEW IX: 2949f.); MÄGISTE (EEW IX: 2949f.) spricht zudem von einem osfi. Stamm.

Als Stamm ist *soke- $\delta a$ anzusetzen. Zum Ableitungssuffix $-\delta A$ und seinen einzelsprachlichen Vertretungen s. LAANEST (1982: 205).

est: e:3, e:4

wot: w: $1, \mathrm{w}: 10$

liv: 1:3, 1:19, 1:23, 1:40

estS: eS:6, eS:9

124 est. sõl|g, gen. -le 'Spange' estS. sõlg', gen. sõlõ id. | liv. suolg id.

fi. solki 'Spange' 
Laut SSA (III: 196) in einigen osfi. und weiteren fiugr. Sprachen belegt; < indoeur. (EES 494); fiugr. (EEW IX: 2955f.)

wot: Die von EES (494) zu den anderen Beispielen hinzugestellte Form wot. sõlk 'Spange' wird in VKS (1200) dem Krewinischen zugeschrieben. WINKLER (1997: 353) interpretiert dieses Wort im Krewinischen als eine estnische Entlehnung und begründet dies u. a. mit dem geringen Vorkommen des Wortes (drei Beispiele) im Wotischen und dem nicht vorhandenen Auslautvokal.

est: e:3

liv: $1: 2,1: 21$

estS: eS:6, eS:9, eS:20

125 est. sõlm, gen. - - 'Knoten' wot. sõlm $\mid u$, gen. - $u$ id. $\sim$ estS. sõlm', gen. sõlmõ id. $\mid \sim$ liv. suolm id.

fi. solmu, solmi 'Knoten'

Laut SSA (III: 196f.) in allen osfi. und einigen weiteren fiugr. Sprachen belegt; fiugr. (EES 494); keine Herkunftsangaben in EEW (IX: 2951)

est: e:3

liv: $1: 2,1: 21$

estS: eS:6, eS:9, eS:20

126 est. sõmer, gen. - $a$ 'Kies, grober Sand' wot. sõmõrõ id. estS. sõm|mõr', gen. -õra id. | liv. sumār 'Krümel, Krume, Korn'

fi. somero 'grober Kies, Geröll'

Laut SSA (III: 197f.) in einigen osfi. Sprachen und im Lp. belegt; osfi.-lp. (EES 494; EEW IX: 2958)

est: e:3

wot: w:1

liv: $1: 6$

estS: eS:9, eS:20

127 est. sõna 'Wort' wot. sõna id. liv. sõnā id. estS. sõna id.

fi. sana 'Wort'

Laut SSA (III: 153f.) in allen osfi. Sprachen belegt; osfi. (EES 494; EEW IX: 2958)

128 est. sõnn, gen. - $i$ 'Stier' estS. sõnn, gen. -i 'Schafbock, Widder, Hammel'

fi. sonni 'Stier'

liv: (Sal) sonn 'Schafbock, Widder' (SSA III: 198; WINKLER/PAJUSALU 2009: 178) 
Laut SSA (III: 198) in den genannten osfi. Sprachen belegt; EES (494) bezeichnet den Stamm als osfi. und erwägt eine Entlehnung, für welche es jedoch keine Quellen gebe.; keine Herkunftsangaben in EEW (IX: 2961)

est: e:3

estS: eS:6

129 est. sõnnik, gen. - $u$ 'Mist, Dünger' estS. sõnnik id.

fi. sonta 'Mist, Dünger'

Laut SSA (III: 199) in einigen osfi. Sprachen und dem Tscher. belegt; osfi.-tscher. oder osfi.-perm. (EES 494); MÄGISTE (EEW IX: 2961f.) setzt einen osfi.-tscher.-?wotj. Stamm sonta an.

est: e: 15

estS: eS:23

130 est. sõre 'undicht, grob' | wot. sorõa 'groß, grob, undicht' estS. sorrõ 'undicht, grob' fi. sorea 'ebenmäßig, schön'

SSA (III: 201) bezeichnet den Stamm als deskriptiv und erwägt für fi. sorea eine Entstehung aus einer möglichen Kontamination von fi. solea 'schlank, rank, astlos' und fi. suora 'gerade, direkt'; ? < indoeur. oder ? onomat. (EES 495); osfi. deskriptiv (EEW IX: 2963)

Als Stamm ist *sore- $\delta a$ anzusetzen. Zum Ableitungssuffix $-\delta A$ und seinen einzelsprachlichen Vertretungen s. LAANEST (1982: 205).

est: e:3, e:4

wot: w: $1, \mathrm{w}: 10$

131 est. sõr $\mid g$, gen. - $a$ 'Klaue' liv. sõ̃rga 'Furche der Klaue' estS. sõrg, gen. sõra 'Klaue' | wot. sorkka 'Klaue'

fi. sorkka 'Klaue, Fuß oder Bein des Paarhufers'

Laut SSA (III: 202) in allen osfi. Sprachen belegt; osfi. (EES 495); keine Herkunftsangaben in EEW (IX: 2964)

est: e:3

liv: $1: 1$

estS: eS:6

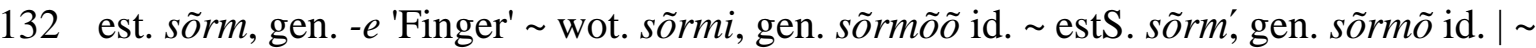
liv. suorm id.

fi. sormi 'Finger' 
Laut SSA (III: 202) in allen osfi. Sprachen, im Lp. und Md. belegt; osfi.-md. (EES 495); osfi.-lp.-md. (EEW IX: 2965f.)

est: e:3

liv: $1: 2,1: 21$

estS: eS:6, eS:9

133 est. sõsar 'Schwester' wot. sõzar id. liv. sõzār id. estS. sõsar'id.

fi. sisar 'Schwester'

< balt. (SSA III: 187; EES 495); < indoeur., wahrscheinlich balt. (EEW IX: 2967f.)

estS: eS:20

134 est. sõstar 'Johannisbeere' wot. sõssar id.

fi. siestar 'Schwarze Johannisbeere'

Laut SSA (III: 173f.) in einigen osfi. Sprachen belegt; osfi. oder osfi.-md. (EES 495);

osfi.-? fiugr. (EEW IX: 2968f.)

wot: w:7

135 est. sõtkas 'Bucephala clangula (Schellente)' estS. sõtkas, gen. .sõtka id.

fi. sotka 'Tauchente'

Laut SSA (III: 204) in einigen osfi. und weiteren fiugr. Sprachen belegt; fiugr. (EES 495f.); urfiugr. (EEW IX:2969)

136 est. sõtkuda 'treten, kneten' wot. sõtkoa/-ua id. estS. .sõkma, II. Infinitiv sõkkuq id. | liv. suotkõ id.

fi. sotkea 'durcheinanderbringen; kneten; zertreten; [...]'

Laut SSA (III: 204f.) in allen osfi. Sprachen belegt; osfi. oder osfi.-perm., vielleicht onomat. (EES 496); osfi.-?syrj. (EEW IX: 2969f.)

est: e:12

wot: w:20

liv: $1: 2,1: 19,1: 22$

estS: eS:7

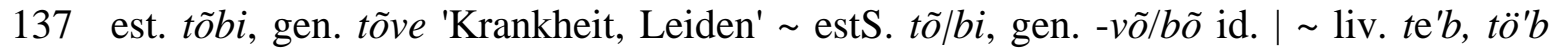
'Krankheit'

fi. ohne Entsprechung

urfi. *topi $<*$ tope (LÄGLOS III: 332) 
Kein Eintrag in SSA; < urgerm. oder urskand., Entlehnung der est. Form zu fi. dial. topi 'Krankheit, Lungenkrankheit' (EES 560); est.-liv. (EEW XI: 3396); fi. topi, welches < est., ? germ. Lehnwort (LÄGLOS III: 332f.)

liv: $1: 21,1: 24,1: 40$

estS: eS:9

138 est. tõbras 'Vieh' wot. tõbraz 'Rentier, Elch' estS. tõbras, gen. .tõpra 'Rind; Vieh' fi. teuras 'Schlachttier' urfi. *tepras / *teuras (LÄGLOS III: 291f.).

? > germ. (SSA III: 288; EEW XI: 3397); < urgerm. (EES 560); germ. Lehnwort (LÄGLOS III: 291f.)

139 wot. tõhtia 'dürfen, können' | est. tohtida 'dürfen' liv. tū'od̂̃ id. estS. toht 'ma, II. Infinitiv .tohtiq id.

fi. tohtia 'wagen, sich trauen' fosfi. *tošt- (SSA III: 303) ? < ieur. (SSA III: 303, EES 535); osfi.-lp.-tscher. (EEW X: 3201) est: e:18 wot: < est. (SSA III: 303) liv: 1:4, 1:19, 1:33; analoge Bildung zu 1:22 estS: eS:7, eS:20

140 est. tõke, gen. tõkke 'Hindernis, Hürde' wot. tõg o, gen. tõkõo 'Fischwehr, Fischzaun' | liv. to'ggõd, to'ugad id.-PL

fi. toe 'Fischwehr; Fischzaun'

< balt. (SSA III: 301; EES 561; EEW XI: 3401)

wot: w:1

liv: 1:19, 1:40; <*tokehet (PosTI 1942: 7)

141 est. tõmmata 'ziehen' wot. tõmmata id. liv. tõmbõ id. estS. .tốmbama id. fi. temmata 'reißen' < balt. (SSA III: 282; EES 561); ? < balt. (EEW XI: 3406) liv: 1:19, 1:23

142 est. tõnguda 'wühlen' wot. tõygata id.

fi. tonkia 'graben, wühlen; durchsuchen'

Deskriptiv (SSA III: 309); osfi. onomat. (EES 562); osfi. deskriptiv (EEW XI: 3410) est: e:12 
143 liv. tỗlza 'stumpf (Waffe, Streitwaffe), ranzig geworden' | est. tülts, gen. - $a$ (Wied.) 'stumpf'

fi. tylsä 'stumpf, nicht scharf, stumpfsinnig'

SSA (III: 345) erwägt eine mögliche Ableitung von fi. tyly 'unfreundlich; rau', welche jedoch nicht gesichert ist.; kein Eintrag in EES; osfi. deskriptiv (EEW XI: 3490)

est: e:1, e:3

liv: $1: 1,1: 17$

144 est. tõri, gen. tõrve 'Horn, Röhre'

fi. torvi 'Hirtenhorn, Horn; Röhre [...]'

< balt. (SSA III: 313; EES 562); Keine Erklärung in EEW (XI: 3416, 3421)

145 est. tõrjuda 'abwehren, verdrängen' estS. tõrjuda id.

fi. torjua 'nicht herankommen lassen, verscheuchen [...]'

SSA (III: 312) setzt fi. tora 'Zank, Streit' als mögliche Variante von fi.-est. torju- an; osfi., osfi.-lp. oder osfi.-md. (EES 562); osfi.-lp.-md. (EEW XI: 3417)

est: e:12

146 est. $t \tilde{o r} \mid s$, -re 'Kübel, Kufe' liv. tỗrdaz id. estS. .tõrdo id.

fi. torto 'längliches Holzgeschirr, Teiggefäß; große Tonne, Zuber' (laut EES 563 dial.)

Kein Eintrag in SSA; osfi. (EES 563; EEW XI: 3419;)

liv: 1:1; $-z$ ist hier wohl analogisch vgl. liv. kêvaz 'Birke' (vgl. LW XXXIX)

147 est. tõru 'Eichel' liv. tô'rmõz id. | wot. turu id.

fi. terho 'Eichel'

SSA (III: 285) stellt eine Verbindung zu fi. terä 'Klinge [...]' her.; EES (562) nimmt eine ungesicherte Variante von est. tera 'Korn; Schneide, Klinge' an. Est. tõru und Entsprechungen werden dort nicht weiter behandelt.; osfi. terho (EEW XI: 3420); KETTUNEN (LW: 415) setzt für liv. ein Derivat an.

est: e:2

wot: ? < est. dial. (SSA III: 285)

liv: $1: 19,1: 43$

148 est. tõrv, gen. - $a$ 'Teer' $\sim$ wot. tõrva id. liv. tỗra id. estS. tõrv, gen. tõrva id.

fi. terva 'Teer'

urfi. *terva (LÄGLOS III: 289) 
< balt. oder < urgerm. (SSA III: 286; EES 563; EEW XI: 3420f.). Eine germ. Entlehnung sei laut MÄGISTE (EEW XI: 3421) unwahrscheinlicher; ? germ. oder balt. Lehnwort (LÄGLOS III: 289f.)

est: e:3

liv: $1: 1$

estS: eS:6

149 est. tõsi, gen. tõe 'wahr; Wahrheit' $\sim$ wot. tõsi, gen. tõ $\tilde{o} \mathrm{id} . \sim$ estS. tôtõ id. $\mid \sim \operatorname{liv} . t^{u} o^{\prime} i z ̌$ id.

fi. tosi 'wahr, wirklich, ernst'

urfi. *tosi <<*tote (LÄGLOS III: 305f.)

? < balt. oder germ. (SSA III: 313, EES 563); osfi.-lp. (EEW XI: 3422ff.); ? germ. oder balt. Lehnwort oder noch älter (LÄGLOS III: 305f.)

liv: $1: 2,1: 24,1: 30,1: 42$

estS: eS:9

150 liv. tõ'v 'Stamm' | est. tüvi, gen. -e id. wot. tüvi, gen. tüvee id. estS. tüvi, gen. -e id.

fi. tyvi 'unteres, dickeres Ende (bes. eines Baumstammes)'

Laut SSA (III: 350) in allen osfi. und einigen weiteren fiugr. Sprachen belegt; fiugr. (EES 572f.; EEW XI: 3506ff.)

liv: $1: 7,1: 40$

151 liv. tõv $\bar{a}$ 'tief' | est. süva id. wot. süvä id. estS. süvä id.

fi. syvä 'tief'

urfi. *süvä $<*$ tivä (LÄGLOS III: 255)

SSA (III: 233) setzt fosfi. *tivä und einen (außer für das Livische gültigen) Lautwandel *ti- > si mit anschließender Labialisierung von $i$ an.; EES (503) geht von einem osfi.lp. oder osfi.-tscher. Stamm, einer möglichen urgerm. Entlehnung oder einer hier nicht einschlägigen Variante von est. tü̈̈ne 'still, ruhig' aus.; osfi.-lp.-tscher.-?md. zu urosfi. tivä (EEW IX: 3018); ? germ. Lehnwort (LÄGLOS III: 255f.)

est: e:1

liv: 1:7, 1:17

152 est. võhk, gen. võha 'Pflanze aus der Familie der Araceae' wot. võhka ' Calla palustris' estS. võhaleht' 'Calla palustris; [...]'

fi. vehka 'Calla, Calla palustris' 
Laut SSA (III: 421) in allen osfi. Sprachen belegt; osfi. (EES 615; EEW XII: 3934) est: e:3

153 est. võl $\mid g$, gen. - $a$ 'Schuld' wot. võlka id. liv. vȭlga id. estS. võlg, gen. võla id. fi. velka 'Geldschuld' Laut SSA (III: 424) in allen osfi. Sprachen belegt; osfi. (EES 616; EEW XII: 3944f.) est: e:3 liv: $1: 1$ estS: eS:6

154 est. võlu 'Zauber' liv. võ'l 'Zauberer, Hexe' estS. võhl, gen. võhlu id. fi. velho 'Hexenmeister, Hexe, Wunderheiler' wohl < russ. (SSA III: 423); < altruss. (EES 616); osfi. (< ? altruss.) (EEW XII: 3947f.) est: e:2; e:7 liv: 1:21, 1:32, 1:43 estS: eS:3, eS:6, eS:19

155 est. võnnu- (in Zusammensetzungen) 'völlig fremd' | wot. ventõizõ̃ id. fi. vento 'völlig fremd' urfi. *vento (LÄGLOS III: 387)

SSA (III: 425) erwägt einen ursprünglich gleichen Stamm wie für fi. vento 'weich, nicht tragend; biegsam; faul [...]', welches ? < urgerm. (SSA III: 425); ? < urgerm. (EES 616f.). Auf eine Verbindung zu fi. vento 'weich, [...]' wird verwiesen, jedoch nicht mit der urgerm. Ableitung in Beziehung gesetzt.; keine Herkunftsangabe in EEW (XII: 3950); germ. Lehnwort (LÄGLOS III: 387f.)

est: e:2

wot: w:1

156 est. võr|d, gen. -ra '[in Zuss.] gleich; Wert, Betrag' wot. (sev)võrta 'Größe, mit der etw. bzgl. Menge, Wert usw. verglichen wird, ebenbürtig (alt), soviel' fi. verta 'Größe, mit der etw. bzgl. Menge, Wert usw. verglichen wird, ebenbürtig (alt)' urfi. *verta (LÄGLOS III: 392)

< (ur)germ. (SSA III: 428f.; EES 617); < germ. (EEW XII: 3951ff.); germ. Lehnwort (LÄGLOS III: 392)

est: e:3

157 est. võr|k, gen. -gu 'Netz' wot. võrkko id. liv. võrgõ id. estS. võrk, gen. võrgu id. fi. verkko 'Netz' 
urfi. *verkko (LÄGLOS III: 391)

< (ur)germ. (SSA III: 428; EES 617); ? osfi. (EEW XII: 3954); germ. Lehnwort (LÄGLOS III: 391f.)

est: e:2, e:3

liv: $1: 19$

estS: eS:3, eS:6

158 est. ? võrkida (Wied.) 'ermatten, erschöpfen' liv. vẹrka 'langsam schleppend (z.B. Krankheit)'

fi. verkkainen 'langsam; gemächlich'

Laut SSA (III: 427f.) in einigen osfi. Sprachen belegt; kein Eintrag in EES; MÄGISTE (EEW XII: 3956) erwägt eine mögliche Ableitung von *võrk oder ungesicherte Verbindung zu est. virge 'munter sein; aufgeweckt', virk 'fleißig, emsig, hurtig', virgutama 'aufmuntern, antreiben'. Liv. stellt er nicht mit est. zusammen.; KETTUNEN (LW 484) stellt eine Verbindung zu fi. verkka 'langsam, gemächlich', verkkaisa id., verkkainen id. her. Wiedemann (1923: 1397) setzt die est. Variante als Mundart von Tartu an.

est: e:18

liv: $1: 1$

159 est. võrsuda 'sprießen' estS. .võrsu|ma, II. Infinitiv -daq id.

fi. verso 'Trieb, Sproß, Schößling', versoa 'sprießen'

Laut SSA (III: 428) in einigen osfi. Sprachen belegt; osfi. oder ? < urindoiran. (EES 617); osfi. (EEW XII: 3956f.)

est: e:2

estS: eS:3

160 est. võsa 'Schössling, Spross' wot. võso id. liv. võzā 'Trieb, Sprössling, Schössling' estS. võso 'Schössling, Spross'

fi. vesa 'Wurzel-, Stocktrieb, Spross, Schoss, Sprößling'

Laut SSA (III: 429) in allen osfi. Sprachen belegt; osfi. (EES 617; EEW XII: 3958f.)

wot: w:18

estS: eS:24

161 est. võtta 'nehmen' wot. võttaa id. liv. võttõ id. estS. .võtma id.

fi. ottaa 'nehmen' 
Laut SSA (II: 276) in allen osfi. Sprachen belegt, in weiteren fiugr. nur ungesichert; EES (618) setzt einen osfi. Stamm mit Verbindung zu einem nicht genannten fiugr. mit Entsprechungen u. a. im Syrjänischen an.; osfi. oder älter (EEW XII: 3960ff.) liv: $1: 19$

\subsubsection{Langvokale}

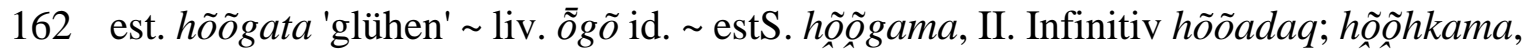
II. Infinitiv hõohadaq id.

fi. ohne Entsprechung

Kein Eintrag in SSA; EES (83) setzt einen onomat. Stamm an und erwägt eine Nähe zu est. ôhkama 'seufzen; ausströmen'; MÄGISTE (EEW II: 430) nimmt einen deskriptiven osfi. Stamm an, welcher in Verbindung zu fi. hehkua 'glühen' stehen könne. Aufgrund der vorausgegangenen Etymologien (s. o.) ist diese Zusammenstellung als ungesichert anzusehen.

liv: 1:19, 1:22, 1:27, 1:33

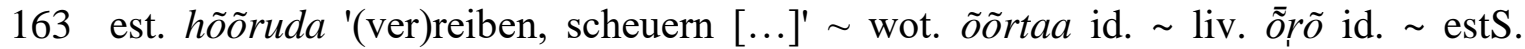
hõõr(d)ma, II. Infinitiv hõõr(d)uq id.

fi. hieroa '(ver)reiben, scheuern [...]'

Laut SSA (I: 160f.) in allen osfi. Sprachen belegt; osfi. oder osfi.-perm. (EES 83); MÄGISTE (EEW II: 432) setzt einen fiugr. Stamm und eine ursprüngliche Form *hēroi an, auf welche das Estnische zurückgehen soll.

est: e:12

wot: w:11

liv: $1: 19,1: 22,1: 27$

estS: eS:7

164 est. kõõm, gen. - a 'Kopfschuppen' liv. kõma 'Knorpelchen' | estS. koomõq, gen. kuumõ; kohmõq, gen. .kohmõ 'Kopfschuppen'

fi. ohne Entsprechung

Kein Eintrag in SSA; keine Herkunftsangabe in EES (204); est.-liv. (?-md.) (EEW IV: 1116)

est: e:3 
165 est. lõog, gen. lõa 'Leine' wot. lõoka 'Strick, Kette' estS. lõ ig, gen. lõia 'Leine'; Mulgi auch: lõogg

fi. lieka 'Pfahl und Strick zum Anbinden des Viehs'

urfi. *lēka (LÄGLOS II: 199)

< germ. (SSA II: 69); < urgerm. (EES 261); osfi. (EEW V: 1428); germ. Lehnwort (LÄGLOS II: 199)

est: e:3

166 est. lõosk, gen. - a 'große Flamme' wot. lõõska 'Feuerzug, Heizkanal'

fi. lieska 'aus dem Ofen schlagende Flamme; Stelle oberhalb der Ofenöffnung'

Deskriptiv (SSA II: 71); osfi., vielleicht onomat. (EES 261); keine Herkunftsangabe in EEW (V: 1430f.). Bei der von MäGISTE (ebd.) angeführten Form liv. li'es̀mô(z) 'Flamme' ist eine Entlehnung aus dem Lettischen anzusetzen, vgl. auch LW (193) und lett. liesma 'Flamme' (ForSSMAN 2010: 129).

est: e:3

167 est. lõots, gen. -a 'Blasebalg' wot. lõotsua 'Bälge treten' liv. lỗtstõ 'keuchen, schnaufen, pusten' estS. lõõts, gen. lõodsa 'Blasebalg'

fi. lietsoa '(mit dem Blasebalg) anfachen'

urfi. *lēcca (LÄGLOS II: 201)

Laut SSA (II: 72) in allen osfi. Sprachen belegt; ? < urgerm. (EES 261); ? osfi. onomat. (EEW V: 1431); ? germ. Lehnwort (LÄGLOS II: 201f.)

est: e:3

liv: 1:19, 1:22

estS: eS:6, eS:15

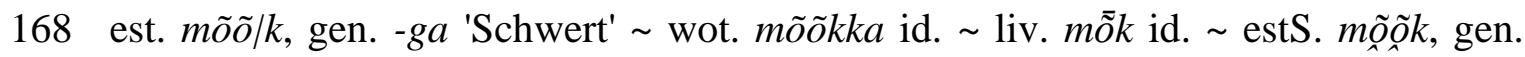
mõ̃oga id.

fi. miekka 'Schwert, Degen [...]'

urfi. *mēkka (LÄGLOS II: 262)

< germ. (SSA II: 165; EEW V: 1606); < urgerm. (EES 293f.); germ. Lehnwort (LÄG-

LOS II: 262)

est: e:3

estS: eS:6

169 est. mõ $\tilde{o} \mid t$, gen. $-d u$ 'Maß, Ausmessung' wot. mõotto 'Maß' estS. mõ õ t, gen. mõ $\tilde{\alpha} d u$ id. 
fi. ohne Entsprechung

urfi. *mētta- (LÄGLOS II: 280)

Kein Eintrag in SSA; < urgerm. (EES 294); MÄGISTE (EEW V: 1607) nimmt eine Ableitung von est. mõ̃ta 'messen' an, welches < germ.; ? germ. oder jüngeres Lehnwort (LÄGLOS II: 280)

est: e:3

estS: eS:6

170 est. $\tilde{h} \nu v(a)$ 'Sterke, Färse' wot. õhva id. liv. ó $u v$ 'Sterke, Färse, junge Kuh (vor dem Kalben)' estS. (h)õhv, gen. (h)õhva 'Sterke, Färse'

fi. hieho 'Färse, Kuhkalb'

Herkunft unklar (SSA I: 160); ? < balt. (EES 625); osfi. (EEW XII: 4014)

wot: w:11

liv: 1:37; Posti (ebd. 245) setzt für das Livische folgende Entwicklung an: *(h)ehvo $>* e$ euho $>$ *evvu $>$ ęcuv

Aufgrund der osfi. Entsprechungen ist ein ursprünglicher Langvokal ansetzbar, vgl.

fi. hieho 'Färse, Kuhkalb'47 ing. hēvikke id. (SSA I: 160).

estS: eS:6

171 est. põ̃on, gen. - $a$ 'Querleiste' liv. põ̃n 'Querleiste, Querholz' estS. põõnn, gen. põ̃̃na 'Querleiste' | wot. piina 'Pfosten Pfeiler'

fi. piena '(Quer)leiste, Quer-, Stützholz'

urfi. *pēna (LÄGLOS III: 54)

< germ. (SSA II: 348; EES 402); ? osfi.-md. (EEW VII: 2300f.); germ. Lehnwort (LÄGLOS III: 54f.)

est: e:3

wot: kar. Einfluss (VKÄ 131)

liv: $1: 21$

estS: eS:6

172 est. rõõs $\mid k$, gen. - $a$ 'frisch, süß, ungesäuert' wot. rõ̃os $k a$ id. liv. rõ̃skõ id. estS. rõ õsk, gen. rõõsa id.

fi. rieska 'frisch, süß, ungesäuert'

< balt. (SSA III: 72; EES 443; EEW VIII:2584)

${ }^{47}$ fi. $i e<* e e$ (HAKULINEN 1979: 38) 
est: e:3

liv: $1: 19$

estS: eS:6

173 est. rõ̃m, gen. $-u$ 'Freude, Vergnügen' $\sim$ liv. $r \overline{\tilde{o} m}$ 'Freude ${ }^{\prime 48} \sim$ estS. rõ õ $\tilde{\alpha} m$, gen. rõ̃mu 'Freude, Vergnügen'

fi. riemu 'Wonne, Freude; Trubel'

Deskriptiv (SSA III: 70f.); osfi. onomat. (EES 442); osfi. onomat. deskriptiv (EEW VIII: 2582f.)

est: e:3

liv: $1: 21$

estS: eS:6

174 est. sõõm, gen. - $u$ 'Schluck, Zug' estS. sõõm, gen. sõõmu id. | liv. sēmda 'Milch' fi. siemaista 'geirig oder schnell trinken, hinunterkippen, -schlürfen' Laut SSA (III: 172) in den genannten osfi. Sprachen und im Md. belegt; osfi.-md. (EES 496); MÄGISTE (EEW IX: 2972f.) setzt einen osfi.-md. Stamm an, welcher auf ein zu -õo- velarisiertes $*-\bar{e}$ - zurückgehe.

est: e:3

liv: KETTUNEN (LW 358f.) erwägt für liv. verschiedene Möglichkeiten: ein deskriptives Wort mit verschiedenen Varianten, ein Wort ohne Etymologie aus der Kindersprache oder eventuell gar aus dem Lettischen entlehnt.

estS: eS:6

175 est. sõ̃r $\mid e$, gen. -me 'Nasenloch, Nüster' wot. sẹr $(a) m e d$ id. liv. sõ̃rmõz id. estS.

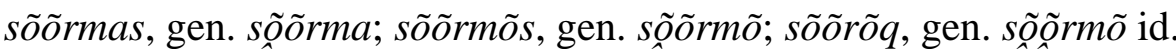

fi. sierain 'Nasenloch, Nüster'

Laut SSA (III: 173) in einigen osfi. Sprachen belegt, im Lp. ungesichert; osfi. oder osfi.-lp. (EES 497); osfi.-lp (EEW IX: 2977)

est: e:16

liv: $1: 19,1: 23$

estS: e: 10

${ }^{48}$ SSA (III: 70f.) nennt für das Wotische noch eine Variante (Kukk) rēmut 'Freuden, lauter Gang'. In VKS und EES ist diese Form nicht zu finden. Da die Etymologie dieser wotischen Entsprechung nicht ausreichend belegt ist, wird sie hier nicht behandelt. 


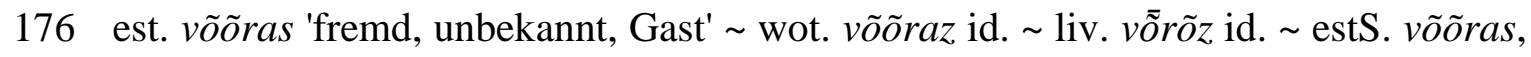
gen. võ õ ra id.

fi. vieras 'fremd, unbekannt; Gast'

SSA (III: 433f.) setzt eine Ableitung von fi. vieri 'Rand, Seite, Kante' an.; EES (618) und MäGISTE (EEW XII: 3962) erwägen eine Ableitung von est. veer 'Rand, Kante'. Die Form est. võ̃ ras samt Entsprechungen wird nicht weiter behandelt (vgl. SSA III: 434; EES 596; EEW XII: 3774), sodass die Ableitungsverhältnisse unklar sind. liv: $1: 19$

\subsubsection{Diphthonge}

177 est. hõim, gen. - $u$ 'Familie, Sippe' wot. õimo id. estS. hõim, gen. hõimo id. $\mid \sim$ liv. aim id.

fi. heimo 'Sippe, Geschlecht [...]'

< balt. (SSA I: 150; EES 82; EEW II: 418f.)

est: e:2, e:3

liv: $1: 27$

178 est. jõhv, gen. -e, -i 'Pferdehaar, Rosshaar' wot. jõv|i, gen. - $i i$ id. liv. jõ'ugi 'aus Rosshaar' estS. jõhv', gen. jõhvi; jõuh, gen. jõuhõ 'Pferdehaar, Rosshaar' fi. jouhi 'Rosshaar'

Laut SSA (I: 243) in allen osfi. Sprachen belegt; osfi. (EES 103); MÄGISTE (EEW II: 581f.) setzt einen osfi.-lp. Stamm an, der auf urosfi. *jouhi zurückgehe.

est: e:3, e:6

wot: w:12

liv: $1: 33,1: 35,1: 37,1: 45$

estS: eS:6, eS:8, eS:17, eS:20

179 est. jõuda 'können, vermögen; Zeit haben' wot. jõutaa id. estS. .joudma, II. Infinitiv (.)joudag; .jõudma, II. Infinitiv (.)jõudaq id. | liv. joudõ 'können, vermögen' fi. joutaa 'frei sein; Zeit haben' Laut SSA (I: 244) in allen osfi. Sprachen belegt; osfi. (EES 103f.); MÄGISTE (EEW II: 587f.) setzt einen urosfi. Stamm *jõuta-, jõude an. liv: $1: 19$ 
180 liv. jõugõ 'Sand' | est. (dial.) hiuk, gen. hiugi 'Bodensatz'

fi. hiukka 'Sandkorn, Stückchen; Partikel [...]'

Laut SSA (I: 168) in einigen osfi. Sprachen belegt; kein Eintrag in EES und EEW est: e:3

liv: $1: 16,1: 19,1: 25$

181 est. jõu|k, gen. -gu 'Bande, Rotte, Horde' wot. jõukku 'Büschel, Menge, Gruppe, Verwandtschaft' estS. jõuk, gen. jõugu 'Bande, Gruppe'

fi. joukko 'Menge, Haufen, Schar, Masse'

urfi. *joukko (LÄGLOS I: 141)

? germ. Lehnwort (SSA I: 243; LÄGLOS I: 141f.); fiugr. (EES 104; EEW II: 589)

est: e:2, e:3

wot: w:4

estS: eS:3, eS:6

182 est. jõulud 'Weihnachten' wot. jõulu id. estS. joul, gen. joulu; jõul, gen. jõulu id.

fi. joulu 'Weihnachten'

< skand. (SSA I: 243; EES 104; EEW II: 589)

183 est. kõi $\mid k$, gen. -ge 'alle' wot. kõikki, gen. kõikõõ id. estS. kõ $\mid i k$ ', gen. -gõ id.

fi. kaikki 'alle'

? < balt. (SSA I: 275; EES 199; EEW IV: 1087f.)

est: e:3

wot: w:1; ? < fi. (EEW IV: 1087f.)

estS: eS:6, eS:9, eS:20

184 est. kõikuda 'sich bewegen, schwanken, schaukeln' estS. .kõikma, II. Infinitiv .kõikuq id. | wot. keikab 'schaukeln-3SGPRÄs'

fi. keikkua 'schaukeln', keikka 'hoch und nach hinten gekrümmt; Kufe einer Wiege $[\ldots]^{\prime}$

urfi. *keikka (LÄGLOS II: 73)

SSA (I: 337f.) erwägt zwei Möglichkeiten: ? < skand. oder onomat. deskriptive Form, welche autochthon osfi. sei.; EES (199) bezeichnet den Stamm als osfi. onomat., erwägt aber auch ein urskand. Lehnwort; osfi. deskriptiv (EEW IV: 1087); ? germ. Lehnwort (LÄGLOS II: 73)

est: e:12

wot: < fi. (SSA I: 338) 
estS: eS:7

185 est. (dial. estS.) kõiv, gen. -u 'Birke' liv. kõuvõ id. | wot. koivu id.

fi. koivu 'Birke'

Laut SSA (I: 386) in allen osfi. und bis in die ural. Sprachen belegt; ural. (EES 199); fiugr.-samoj. (EEW IV: 1088)

est: e:3

wot: < fi. (SSA I: 396)

liv: $1.9,1: 19$

186 est. kõu, gen. -e 'Donner' estS. kõvve id.

fi. kouko 'Gespenst, Spuk [...]'

< balt. oder < indoeur. (SSA I: 414); EES (203) setzt eine verkürzte Stammvariante von est. kõuk 'Ahn, Vorfahre' an, welches < balt.; ? < balt. (EEW IV: 1113)

est: e:3

estS: eS:8, eS:16

187 est. lõigata 'schneiden' wot. lõikata id. estS. .lõikama, II. Infinitiv lõigadaq id.

fi. leikata 'schneiden'

urfi. *leikka- (LÄGLOS II: 189)

$?<$ germ. Die Annahme eines gemeinsamen ural. Stammes wird aufgrund der lautlichen Gestalt der osfi. Entsprechungen in SSA (II: 59) abgelehnt; ? < urgerm. (EES 258); osfi.-?samoj. (EEW V: 1410f.); kaum germ. Lehnwort (LÄGLOS II: 189f.)

188 est. lõim, gen. -e 'Weberkette' $\sim$ wot. lõim $\mid i$, gen. -õ id. estS. lõim', gen. lõimõ id. | liv. lūoima id.

fi. loimi 'Kette (beim Weben, in einem Gewebe); Pferdedecke'

Die etymologischen Wörterbücher (SSA II: 87; EES 258) setzen eine Ableitung von fi. luoda 'erschaffen, schöpfen' bzw. est. looma id. an. Die Formen est. lõim und wot. lõimi werden dort nicht behandelt (SSA II:105; EES 249f.).; osfi.-md. (EEW V: 1411) est: e:3

liv: $1: 8$

estS: eS:6, eS:9, eS:20

189 est. lõu|g, gen. - $a$ 'Kinn' wot. lõuka id. liv. lõga id. estS. lõug, gen. lõvva id.

fi. leuka '(Unter)kiefer, Kinn'

? < balt. (SSA II: 66; EEW V: 1423); < balt. (EES 260)

est: e:3 
liv: $1: 15$

estS: eS:6, eS:8, eS:16

190 est. lõu|gas, gen. - $k a$ 'gemauerte Seite des Aschenloches vor der Ofenmündung (als Sitz dienend), Ecke, Krümmung, Meerbusen' estS. lõugõq, gen. .lõukõ id. | ? liv. lōkandõks 'Eisloch'

fi. loukko 'Loch, Winkel'

Laut SSA (II: 96) in einigen osfi. Sprachen belegt; osfi. (EES 260); osfi. (-fiugr.samoj.?) (EEW V: 1424)

liv: $1: 14,1: 19$

estS: eS:9

191 liv. lõuglõ 'gleiten; stolpern, straucheln' | est. liuguda 'gleiten' wot. liukua id. fi. liukua 'gleiten'

SSA (II: 84) erwägt einen möglichen Zusammenhang mit fi. lipua 'gleiten'.; osfi. (EES 244); MÄGISTE (EEW IV: 1331f.) setzt est. liuguda als Ableitung von est. liug 'Gleiten, Glitschen' an, welches osfi. sei.

liv: 1:16, 1:19, 1:46

192 est. lõuna 'Süd, Mittag, Mittagessen' wot. lõunad id. liv. lõ̃nag id. estS. lõunõq, gen. .lõuñ; lõunaq, gen. .lõuna; lõuna|k, gen. -gu id.

fi. lounas 'Südwest(en) [...]; Mittag; Mittagessen'

Laut SSA (II: 97) in allen osfi. Sprachen belegt; osfi.-perm. (EES 260); MÄGISTE (EEW V: 1425f.) setzt einen fiugr. Stamm an und erwägt beim Estnischen eine Ent-

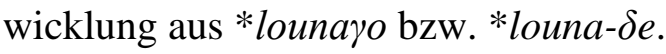

wot: $-D$ ist der Marker des Nominativ Plural (ARISTE 1968: 37)

liv: $1: 13$

estS: eS:9

193 est. mõis, gen. - $a$ 'Gut, Landgut' $\sim$ wot. mõiza id. estS. .mõisa id. | liv. moizõ/muoizə id.

fi. moisio 'kleiner umzäunter Acker; Waldwiese; Herrenhof [...]'

Laut SSA (II: 169) in einigen osfi. Sprachen belegt; osfi. (EES 291; EEW V: 1591)

est: e:3

liv: 1:10, 1:19

194 est. mõista 'verstehen, begreifen' wot. mõissaa id. estS. .mõistma, II. Infinitiv .mõistaq id. | liv. moistə/muoistə 'verstehen, bemerken' 


\section{fi. muistaa 'sich erinnern'}

fiugr. muja- (UEW I: 284)

SSA (II: 174f.) und MÄGISTE (EEW V: 1592) setzen einen Urstamm *muja- an, aus welchem es eine Entwicklung *mujista- > muista- gegeben habe.; fiugr. (EES 291). liv: 1:10, 1:19

195 liv. mō̄ka 'Wurst, Würstchen' | est. mau|k, gen. -gu 'dicke Wurst' wot. mauttšsi 'Darm' estS. maugo 'dicke Wurst' fi. maukku 'Eierstock beim Fisch, Schwimmblase' *maukku / *moukku (LÄGLOS II: 256) ? < skand. (SSA II: 155); ? < urgerm. oder skand. oder onomat. osfi. (EES 278); MÄGISTE (EEW V: 1518) erwägt, dass es sich um einen deskriptiven osfi. Stamm oder ? < skand. handeln könne; ? germ. Lehnwort (LÄGLOS II: 256f.)

wot: w:6

liv: 1:12; danach ist wohl ein Lautwandel $-\bar{o}->-\overline{\tilde{O}}-$ ansetzbar.

196 est. nõi|d, gen. - a 'Zauberer, Hexe' wot. nõita id. estS. nõid, gen. nõia id. fi. noita 'Zauberer, Hexe' Laut SSA (II: 227f.) in allen osfi. Sprachen und im Lp. belegt; fiugr. (EES 324; EEW VI: 1761)

est: e:3 estS: eS:6

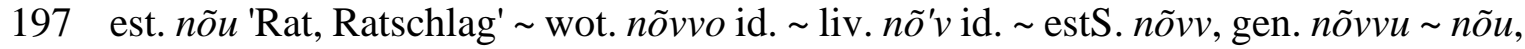
gen. nõvvo id.

fi. neuvo 'Rat; Art und Weise; Ratschlagung [...]'

Laut SSA (II: 215) in allen osfi. Sprachen belegt; osfi. (EES 326; EEW VI: 1770f.) est: e:3, e:11

wot: w:2

liv: $1: 40$

estS: eS:8

198 est. nõuda 'verlangen, fordern' estS. nõudma, II. Infinitiv (.)nõudaq id. fi. noutaa 'holen'

SSA (II: 234) setzt einen ursprünglichen Stamm fi. *nou- an.; ural. (EES 326); MÄGISTE (EEW VI: 1772) setzt einen fiugr. oder ural. Stamm *novta- an. 
199 est. oige 'recht, richtig' wot. oikõa 'recht, richtig, grade' liv. ôigi 'recht, richtig' estS. .õigõ id.

fi. oikea 'rechte/r/s; recht, richtig; echt, wahr [...]'

Laut SSA (II: 260) in allen osfi. Sprachen und im Lp. belegt, darüber hinaus unsicher; osfi.-lp. oder osfi.-wolg. (EES 625); osfi.-lp.-(?)wolg. (EEW XII: 4015)

Als Stamm ist *oike- $\delta a$ anzusetzen. Zum Ableitungssuffix - $\delta A$ und seinen einzelsprachlichen Vertretungen s. LAANEST (1982: 205)

est: e:3, e:4

wot. w:1, w:10

liv: ? < est. (Posti 1942: 33; SSA II: 260; EES 625; LW 51); KETTUNEN (LW 51) erwägt zudem < *oikinen oder eine analoge Form.

estS: eS:9

200 est. õis, gen. õie õile, gen. õilme 'Blüte' | wot. ed'jelmoled'd'ôlmo 'Blütenstaub, Pollen' liv. èdrõm 'Blüte' estS. häilmü, häiermü 'Blühen, Blüte'; häidseq, gen. .häits(m)e; häierm; häielm; häelm 'Blüte'

fi. hedelmä 'Frucht'

SSA (I: 150f.) setzt eine Ableitung von fi. heitiä 'blühen (Roggen, Gerste)' an, welches wohl < balt.; EES (625) nennt als ursprüngliche Form est. ois, welches < balt.; Für est. õile setzt MÄGISTE (EEW XII: 4018) urosfi. *heidelmä an, was veraltet sei. $\mathrm{Zu}$ est. õis nennt er (ebd. 4019) früh-urosfi. *šeiti und verweist auf balt. Einfluss.

est: e:3

wot: w:1

liv: $1: 19$

201 est. oud(ne) 'unheimlich'

fi. outo 'fremd, unbekannt, ungewöhnlich [...]'

Laut SSA (II: 277) in einigen osfi. Sprachen belegt; osfi. (EES 627); osfi.-lp. (EEW XII: 4038)

est: e:17

202 est. põigata 'abbiegen' wot. põitši '(postp.) seit, ab (zeitlich)'

fi. poiketa 'abbiegen; abweichen'

SSA (II: 385) erwägt den gleichen Urstamm wie für fi. poikki 'über, ab, entzwei' und fi. pois 'weg, fort, davon', nennt diesen jedoch nicht.; osfi. (EES 398); MÄGISTE (EEW VII: 2273f.) erwägt einen osfi. Stamm *poikke- (? poikki-). 
wot: w:6

203 est. põimida 'flechten, winden' wot. põimia 'sammeln, pflücken' | liv. pöimó 'flechten, winden'

fi. poimia 'pflücken'

Laut SSA (II: 386) in allen osfi. Sprachen und im Lp. belegt; osfi.-lp. (EES 398; EEW VII: 2274f.)

liv: 1:19; analoge Bildung zu 1:22

204 est. põu|d, gen. - $a$ 'Dürre' wot. põuta 'trockenes, sonniges Wetter; Sonnenschein; Dürre' liv. põ̃da 'neblig, schwül' estS. põud, gen. põvva 'Dürre'

fi. pouta 'trockene Witterung, schönes Wetter'

? > balt. oder urbalt. (SSA II: 408); ? < balt. (EES 402); osfi.-lp. (EEW VII: 2299f.)

est: e:3

liv: $1: 13$

estS: eS:6, eS:8, eS:16

205 est. rõivas 'Kleid, Kleidung' estS. rõivas, gen. .rõiva id. | wot. roivaz '(Flachs-, Stroh-) Bündel'

fi. roivas 'Hanf- oder Flachsbündel, -garbe'

Laut SSA (III: 88) in allen osfi. Sprachen belegt; osfi. (EES 441); wahrscheinlich osfi. (EEW VIII: 2573f.)

206 est. rõu|k, gen. -gu '(auf Reitern, Reutern aufgeschichteter) Klee- oder Kornschober' wot. rõukko id. estS. rõuk, gen. rõugu id.

fi. roukko 'Eishaufen, sich am Ufer schichtendes, Lufträume aufweisendes Eis; Haufen'

urfi. *roukka / rö̈̈kkä / *roukko / *rö̈̈kkö / *roukk(i)jo (LÄGLOS III: 169)

wohl < germ. (urskand.), die etymologischen Wörterbücher erwägen zudem eine Vermischung mit est. rõu|k, gen. - gu 'Stütze des Wagenleiters' (SSA III: 97; EES 442); ? skand. (EEW VIII: 2579); ? germ. Lehnwort, Variation im osfi. Vokalismus sei bereits früh entstanden (LÄGLOS III: 169f.).

est: e:2, e:3

estS: eS:3, eS:6

207 est. rõu|k, gen. -gu 'Stütze des Wagenleiters'

? fi. roukku 'Querbalken auf dem Arbeitsschlitten [...]; Weidenband [...]' 
Laut SSA (III: 97) in den genannten osfi. Sprachen belegt; ? osfi. (EES 442); keine Herkunftsangabe in EEW (VIII: 2579)

est: e:3

208 est. sõim, gen. -e 'Krippe' wot. sõim $\mid i$, gen. -õo '(Pferde)krippe'

fi. soimi '(Futter)krippe'

Die etymologischen Wörterbücher erwägen eine Ableitung vom gleichen Stamm wie fi. syödä 'essen' bzw. est. sööma id. (SSA III: 194; EES 493; EEW IX: 2951). Dort werden est. sõim und Entsprechungen nicht behandelt (vgl. SSA III: 234f.; EES 500f.; EEW IX: 2999-3002); MÄGISTE (EEW IX: 2951) bezeichnet die Sippe ferner als osfi. est: e:3

wot: w:1

209 wot. sõisa 'stehen' | est. seista id. estS. .saisma, II. Infinitiv (.)saistaq id. fi. seisoa 'stehen'

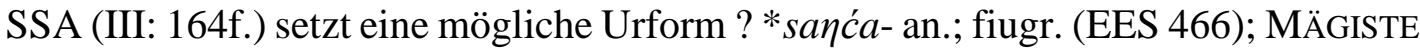

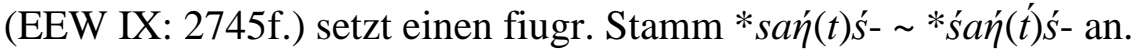

estS: eS:4

210 est. sõita 'fahren' wot. sõittaa 'fahren; schelten' liv. selitz 'jm. etw. vorwerfen' estS. .sõitma, II. Infinitiv .sõitaq 'fahren'

fi. soida '(er)klingen, (er)schallen [...]'

? onomat.-deskriptiv (SSA III: 193); osfi.-lp. onomat. oder fiugr., im Falle eines fiugr. Stammes habe sich aus der ursprünglichen Bedeutung '(er)klingen, (er)schallen' die Bedeutungen 'fahren' und '(sich) streiten' entwickelt (EES 493f.); MÄGISTE (EEW IX: 2953f.) nimmt eine onomat. osfi.-lp.-fiugr. Form mit Stamm *soje- an. liv: $1: 19$

211 liv. sõitõ 'können, vermögen' | est. suuta id. estS. sutma, II. Infinitiv suttaq id. fi. suittaa 'können; vermögen, Zeit haben [...]'

Die etymologischen Wörterbücher erwägen eine mögliche Ableitung von suи 'Mund, Maul' (SSA III: 208; EES 492; EEW IX: 2943). Fi. suittaa/est. suuta und Entsprechungen werden dort nicht behandelt (SSA III: 223f.; EES 491; EEW IX: 2933ff.). liv: 1:19, 1:22

212 est. sõuda 'rudern' wot. sõutaa id. liv. sõudõ id. estS. .sõudma, II. Infinitiv (.)sõudaq id.

fi. soutaa 'rudern' 
Urstamm * sū $\gamma$ - ?? < indoeur. (SSA III: 205); ? < indoeur. (EES 496); MÄGISTE (EEW IX: 2970f.) setzt eine Entwicklung urslav. > frühurfi. an.

liv: $1: 19$

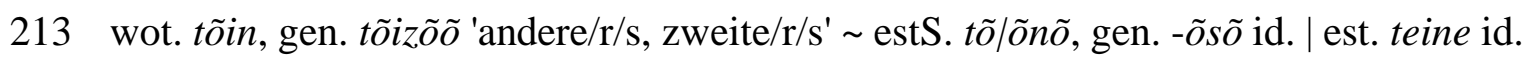
$\sim$ liv. tuoi id.

fi. toinen ' andere/r/s, zweite/r/s'

Die etymologischen Wörterbücher setzen eine Ableitung vom Pronomen fi. tuo 'jener' bzw. est. too id. an (SSA III: 304; EES 522; EEW X: 3116ff.). In SSA (III: 327f.) wird eine osfi. Urform *tō angenommen. Die verwandten Sprachen sollen auf deren wechselnde Vertretung hinweisen, vgl. *to-, *tō-, *tu-. Fi. toinen mit Entsprechungen wird dort nicht behandelt.; EES (538) setzt eine ural. Form mit abweichender Entwicklung der Ableitung teine an (<*toi-nen).; MÄGISTE (EEW X: 3224) nimmt ural. Stämme * to, *tu und *tō an, behandelt est. teine und Entsprechungen jedoch nicht. wot: Apokope ist im Wotischen nicht lautgesetzlich (VKÄ 145f.) liv: $1: 8$ estS: eS:5, eS:9

214 est. tõug, gen. tõu 'Sommersaat, -korn' wot. tõuko id. estS. tõug, gen. tõvvu id. fi. touko 'Aussaat (im Frühjahr); heranwachsendes Getreide' Laut SSA (III: 315) in einigen osfi. und weiteren fiugr. Sprachen belegt; ? < uriran. (EES 563); MÄGISTE (EEW XI: 3429f.) setzt einen fiugr. Stamm *tovkV- an.

est: e:3

estS: eS:8, eS:16

215 est. tõugata 'stoßen, schieben' wot. tõukata id. ? liv. tęvk, tęuk 'stoßen, stampfen' | estS. .toukama, II. Infinitiv tougadaq; .tuukama, II. Infinitiv tuugadaq [...] 'stoßen, schieben'

fi. toukata 'reißen, zupfen; schubsen [...]' deskriptiv (SSA III: 315); osfi. onomat. (EES 564; EEW XI: 3431f.) liv: $1: 13$

216 est. tõu|k, gen. - gu 'Larve, Wurm' wot. tõukka id.

fi. toukka 'Larve, Raupe; Wurm'

Laut SSA (III: 315) in einigen osfi. Sprachen belegt; osfi. (EES 564); keine Herkunftsangabe in EEW (XI: 3431)

est: e:3 
217 liv. tõurõz 'teuer'

fi. tyyris 'teuer'

urfi. *tiuris / *tiüris (LÄGLOS III: 328)

< germ. (SSA III: 351); kein Eintrag in EES und EEW; germ. Lehnwort (LÄGLOS

III: 328)

liv: $1: 16,1: 19$

218 est. $v \tilde{o} i$ 'oder' $~$ wot. või id. liv. või id. | estS. vai id. (EES 615)

fi. vai 'oder'

SSA (III: 389) erwägt verschiedene Erklärungen, aufgrund einer hohen Anzahl an osfi. Partikeln, die sich miteinander vermischt haben, sei ein Ursprung schwer zu ermitteln und die Erklärungen unsicher.; EES (615) setzt einen osfi. Stamm an und erwägt eine Verkürzung eines von vielen Stämmen; osfi. (EEW XII: 3936)

219 est. või 'Butter' wot. või id. liv. võ̃idag id. estS. võid, gen. võiu id.

fi. voi 'Butter'

fiugr. woje 'Fett, Fettstoff' (UEW I: 578)

SSA (III: 467) und EES (615) erwägen zwei Herleitungen. Die fiugr. Wörter wurden oft mit denen aus Turksprachen verglichen. Auch eine Entlehnung aus dem Urar. wird bedacht. EES (615) zufolge sei der Stamm ferner fiugr.; fiugr. (EEW XII: 3936f.)

liv: 1:11; KeTtUNEN (LW 481) verweist auf fi. voide 'Schmiere', was auf eine Ableitung hindeutet (zu fi. vgl. HAKULINEN 1979: 190f.)

estS: s. liv.

220 liv. vęih '(Interjektion, z.B. zum Schrecken)' | est. voi (Wied.) '(Schmerz ausdrückend; Interjektion)'

fi. voi 'ach!, oh!; au! [...]'

Laut SSA (III: 467) in einigen osfi. Sprachen belegt; kein Eintrag in EES; keine Herkunftsangaben in EEW (XII: 3900)

liv: $1: 11$

221 est. võida 'können' wot. vęija/võitaa id. liv. võidõ id. estS. .võima, II. Infinitiv .võidaq id.

fi. voida 'können'

Laut SSA (III: 467f.) in allen osfi. Sprachen belegt; osfi. (EES 616; EEW XII: 3939ff.)

liv: 1:11, 1:19 


\subsubsection{Sonderfälle}

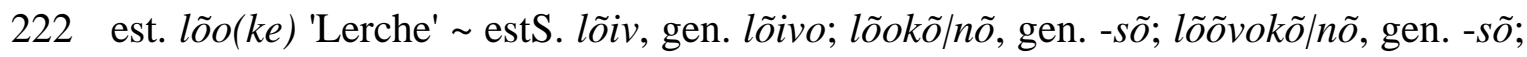
lõivokõ|nõ, gen. -sõ id.

fi. leivo 'Lerche'

urfi. *leivo (LÄGLOS II: 190)

? < germ. (SSA II: 61); < urgerm., onomat. (EES 259); MÄGISTE (EEW V: 1419) erwägt einen deskriptiven Stamm oder eine mögliche Entlehnung von osfi. leivo(i), welches < ? germ.; germ. Lehnwort (LÄGLOS II: 190f.)

est: e:11

estS: eS:5, eS:9

223 wot. õohko 'Distel' | est. ohakas id. liv. ? ū'ogõz/ūogaz/u'ogəz 'Ährenspitze, Dorn; kleine Gräte' estS. uhak; (.)ohtjas; (.)uhtjas; (.)ohk'mas; (.)uhk'mas; (.)oht'mas; (.)uht'mas; uhas 'Distel'

fi. ohdake 'Distel'

SSA (II: 259) setzt einen Urstamm *ohta an, welcher ? < balt. (SSA II: 259); < balt. (EES 334); osfi. (EEW VI: 1815)

liv: $1: 2,1: 19,1: 33$

estS: eS:1

224 est. tõusta 'aufstehen, sich erheben' wot. nõisa 'anfangen, beginnen' liv. nūzõ 'aufstehen, sich erheben' estS. nõs|oma, II. Infinitiv -taq id.

fi. nousta '(auf)steigen, aufstehen, sich erheben'

Laut SSA (II: 234) in allen osfi. Sprachen belegt; osfi. (EES 564; EEW XI: 3433ff.) est: EES (564) setzt - $n$ - > - $t$ - in nordestnischen Dialekten an; ? regressive Assimilation noust- > toust- (EEW XI: 3435)

wot: w:3

liv: $1: 19$

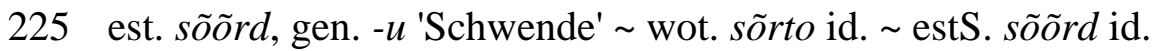

fi. sortaa 'umwerfen, zum Einsturz bringen; niederschlagen [...]'

urfi. *sorta- (LÄGLOS III: 248) 
Laut SSA (III: 203) in einigen osfi. Sprachen belegt; EES (496f.) setzt einen osfi. Stamm an, erwägt aber ebenso eine germ. Entlehnung, für welche jedoch keine passenden Quellen vorliegen.; keine Herkunftsangaben in EEW (IX: 2975f.); kaum germ. Lehnwort (LÄGLOS III: 248f.)

est: e:3

estS: eS:6

226 est. tõotada 'versprechen, geloben' wot. tõivoa 'vorhersagen' liv. tõitõ 'versprechen, geloben' estS. .too|tama, II. Infinitiv -taq; toovotama, II. Infinitiv -taq; .tõo|tama, II. Infinitiv -taq [...] id.

fi. toivoa 'hoffen, jemandem etw. wünschen'

Laut SSA (III: 305) in allen osfi. Sprachen und im Lp. belegt; ? < urindoiran. (EES 562); MÄGISTE (EEW XI: 3412) setzt eine Ableitung von urosfi. toivo an.

est: e:11

liv: $1: 19,1: 22$

estS: eS:12 
5.

Analyse

\section{1}

Zur Vorgehensweise

Im vorliegenden Kapitel erfolgt eine genauere Betrachtung des zusammengestellten Korpus im Hinblick auf die Einzelsprachen. Da sich die meisten der früheren Arbeiten ausschließlich mit dem standardestnischen $\tilde{o}$ beschäftigen und der estnische Teil des Korpus den größten Anteil ausmacht, wird innerhalb der einzelnen Unterkapitel immer zunächst mit der Betrachtung dieser Sprache begonnen. Die anderen Sprachen folgen anschließend in gleicher Reihenfolge wie bei der Darstellung der zu untersuchenden Belege. Die Abfolge der Analysegruppen orientiert sich ebenso an der aus dem Korpus bewährten Reihenfolge. In den einzelnen Kapiteln erfolgt die Darstellung der Lautwandel nicht nach alphabetischer Reihenfolge, sondern aufgrund ihrer Relevanz. Zunächst werden also die Lautwandel behandelt, denen eine hohe Anzahl an Belegen zuzuordnen ist. Lautwandel, die nur eine marginale Vertretung vorweisen, werden in einem späteren Teil der Analyse zusammengestellt. Unter den Sonderfällen gibt es keine konkreten Gruppierungen, sie werden als Einzelfälle betrachtet und für sich diskutiert.

Jedem dieser Unterkapitel geht eine statistische Auswertung der Belege voraus, einerseits zu Beginn ganz allgemeiner Natur, in den einzelnen Abschnitten auf den jeweiligen Lautwandel präzisiert.

\subsection{Zur Datenlage}

Insgesamt wurden 226 einschlägige Belege ${ }^{49}$ untersucht, die sich ohne Berücksichtigung weiterer Kriterien zunächst gemäß der folgenden Tabelle auf die unterschiedlichen Kategorien verteilen:

$\begin{array}{ll}\text { Kurzvokale } & 161 \\ \text { Langvokale } & 15 \\ \text { Diphthonge } & 45 \\ \text { Sonderfälle } & 5\end{array}$

\footnotetext{
${ }^{49}$ Ein Beleg bezeichnet an dieser Stelle nicht nur ein einzelnes Wort. Er kann aus mehreren Wörtern unterschiedlicher Sprachen bestehen. Diese stehen in einem etymologischen Zusammenhang miteinander. Nicht immer sind alle Wörter dabei einschlägig.
} 
Eine Tendenz zur Verteilung der Lautwandel ist hierbei bereits sehr deutlich erkennbar: Die Kurzvokale haben eine Entwicklung zu $\tilde{o}$ am häufigsten vollzogen, während sie bei den Langvokalen selten eintritt. Schlüsselt man diese Werte auf, ergibt sich für die einschlägigen Belege der Einzelsprachen die nachfolgende Darstellung:

\begin{tabular}{lccccc} 
Sprache & \multicolumn{5}{c}{ Anzahl der einschlägigen Belege im Korpus } \\
& gesamt & Kurzvokale & Langvokale & Diphthonge & Sonderfälle \\
Estnisch & 185 & 129 & 15 & 37 & 4 \\
Wotisch & 147 & 103 & 10 & 30 & 4 \\
Livisch & 85 & 55 & 11 & 19 & 0 \\
Südestnisch & 144 & 99 & 13 & 30 & 4
\end{tabular}

Die Darstellung zeigt, dass die Lautwandel im Estnischen am häufigsten auftreten, während das Livische kaum halb so viele einschlägige Belege vorweist. Dieses Verhältnis liegt in nahezu allen Bereichen vor. Auch decken sich die Resultate aus der Tabelle mit dem Verhältnis der Gesamtübersicht. Die Kurzvokale sind in allen Sprachen die stärkste Vertretung, die Langvokale die geringste, wenn man von den Sonderfällen absieht, die eine eigene Rolle einnehmen. Die Auswertung weiterer Daten, die sich auf die Einzelsprachen und das lautliche Umfeld der jeweiligen Belege beziehen, erfolgt in den entsprechenden Kapiteln.

\section{$5.3 \quad$ Kurzvokale}

\subsubsection{Statistische Auswertung}

Im Folgenden werden die Daten der Kurzvokale für die Einzelsprachen statistisch aufbereitet. Die Verteilung der Kurzvokale wurde bereits anhand der vorausgegangenen Tabelle in Kapitel

\section{2 dargelegt.}

Betrachtet man die statistische Verteilung der Lautwandel genauer, wird deutlich, dass zwei Vokale in dieser Kategorie eine Art Zentrum bilden, nämlich $* e$ und $* o$. Eine Vertretung anderer Vokale (z.B. $* a,{ }^{*} i,{ }^{*} u$ ) als ursprünglicher Laut der ersten Silbe liegt nur marginal vor. Diese Erkenntnis deckt sich mit den bisherigen Angaben anderer Forscher. 


\subsubsection{Estnisch}

Unter die 129 einschlägigen estnischen Belege fallen 51, die in der ersten Silbe auf *e zurückgehen. Ein ursprüngliches $*_{o}$ liegt bei insgesamt 71 Belegen vor, auf $* a$ gehen drei zurück sowie zwei auf $* u$. Bei weiteren zwei Belegen sind die ursprünglichen Vokale der ersten Silbe unbekannt und auch unter Zuhilfenahme der Etymologien nicht rekonstruierbar. Die Verteilung der estnischen Belege anhand ihrer ersten Silbe mit Kennzeichnung der Vokale der Folgesilben stellt das nachfolgende Diagramm dar. Dabei ist besonders die Häufigkeit von * $e$ und $* a$ in den nichtersten Silben hervorzuheben. Sie machen in dieser Position die stärksten Vertretungen aus. Im Diagramm sind lediglich die Belege des Korpus erfasst, in denen $\tilde{o}$ in der ersten Silbe auftritt. Jene, die für den Lautwandel nicht einschlägig sind, werden in dieser Darstellung nicht berücksichtigt.

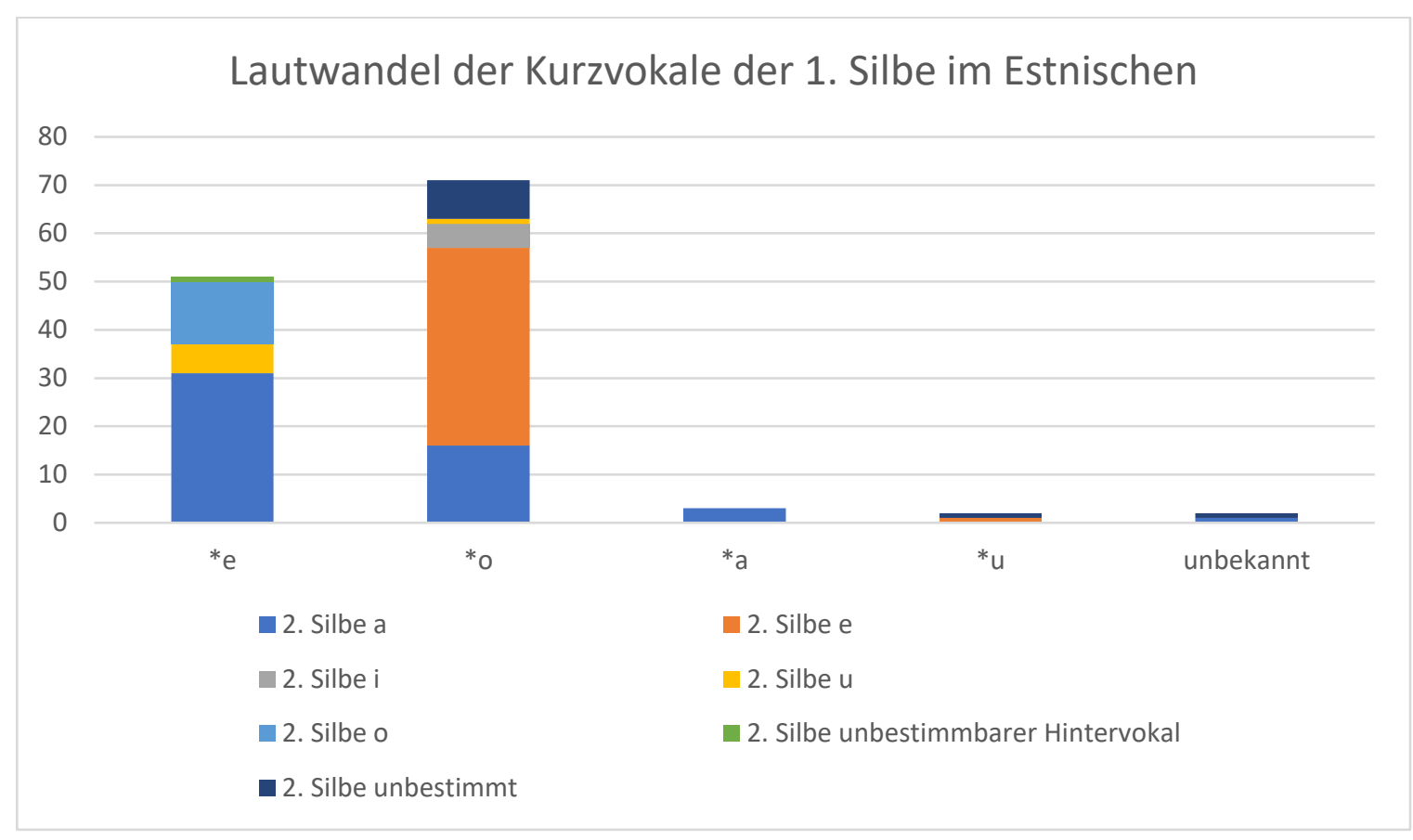

\subsubsection{Wotisch}

Im Wotischen lässt sich eine ähnliche Verteilung wie im Estnischen festhalten. Insgesamt sind 103 der Belege im Fall der Kurzvokale einschlägig. Von ihnen entfallen 32 auf * $e$ in der ersten Silbe, auf $* o$ gehen 67 zurück. Bei drei Belegen existierte ursprünglich $* a$ in dieser Position, auf * $u$ entfällt ein Beleg. Dargestellt werden kann die Verteilung der einschlägigen Belege mitsamt der zweiten Silbe, wie das folgende Diagramm zeigt. Auch im Wotischen sind in der 
nichtersten Silbe die stärksten Vertretungen $* a$ und $* e$. Nicht einschlägige Belege, die im Korpus auftreten, werden auch hier nicht berücksichtigt, Belege, in deren zweiter Silbe $\tilde{o}$ auftritt, wurden zu ihrer historischen Vertretung hinzugezählt.

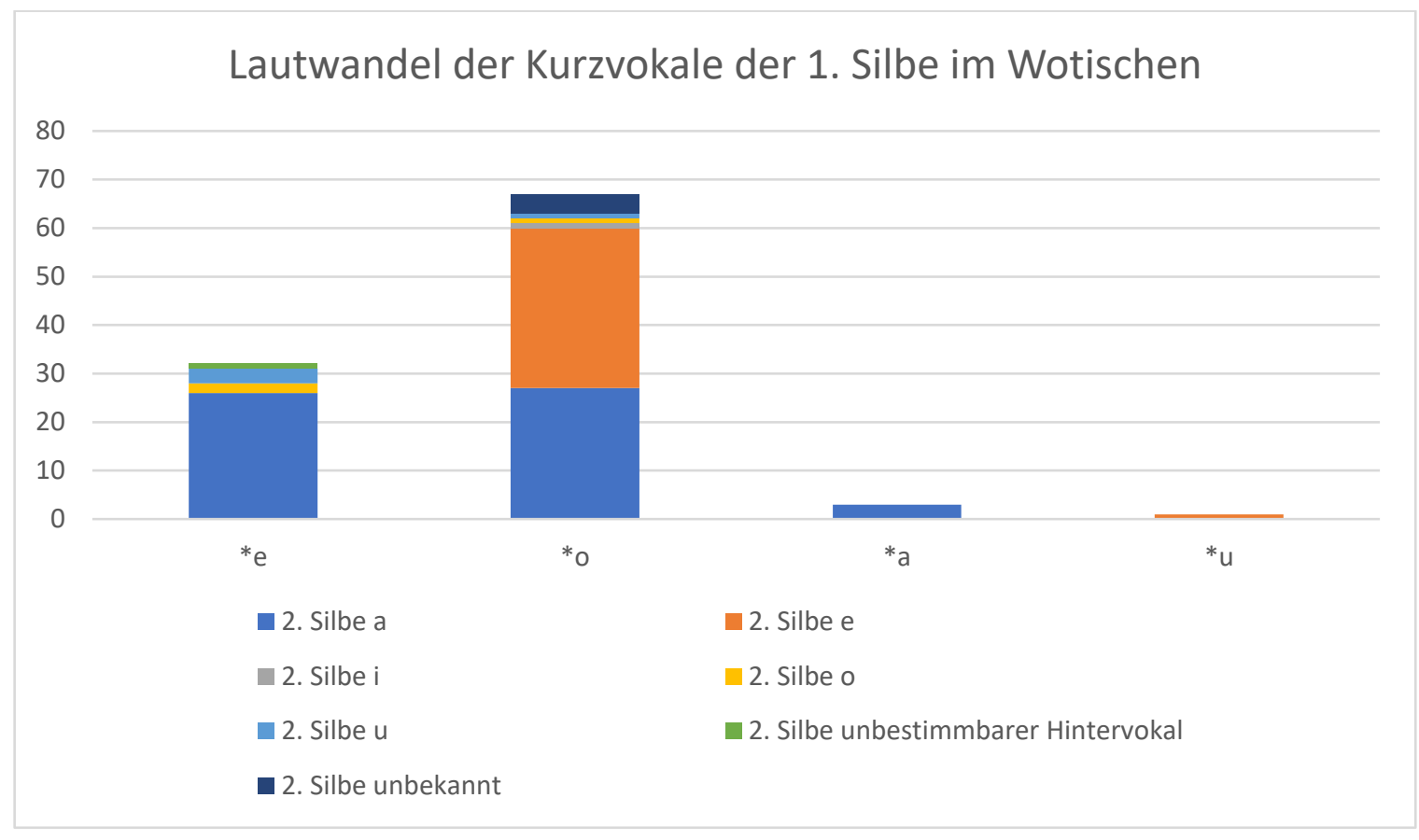

\subsubsection{Livisch}

Das Livische weist mit nur 55 einschlägigen Belegen im Korpus die kleinste Vertretung in der Kategorie der Kurzvokale auf. Auf $* e$ in der ersten Silbe entfallen dabei 36 Belege, auf $*_{o}$ lediglich neun. Hinzu kommen fünf, die auf *y zurückgehen. Bei einem Beleg liegt ursprünglich $* a$ vor, eine gleiche Anzahl findet sich jeweils bei $* i$ und $* \ddot{o}$. Bei zwei Belegen ist der ursprüngliche Erstsilbenvokal unbekannt. Das folgende Diagramm soll die Verteilung im Livischen darstellen, wobei hier ebenfalls die Vokale der zweiten Silbe ${ }^{50}$ berücksichtigt werden, Belege aus diesem Korpus ohne Einschlägigkeit hingegen nicht. Es zeigt sich dabei, dass sich die alte Vertretung der Laute vor dem Lautwandel im Livischen über ein breiteres Spektrum verteilen. Mit Ausnahme des Lautwandels * $e>\tilde{o}$, der die mit Abstand größte Anzahl an Belegen in dieser Sprache zeigt, kann kaum von einer hohen Datenmenge gesprochen werden.

\footnotetext{
${ }^{50}$ Auch für das Livische gilt, dass die Belege mit $\tilde{o}$ in nichterster Silbe zu ihren historischen Vertretungen gezählt wurden.
} 


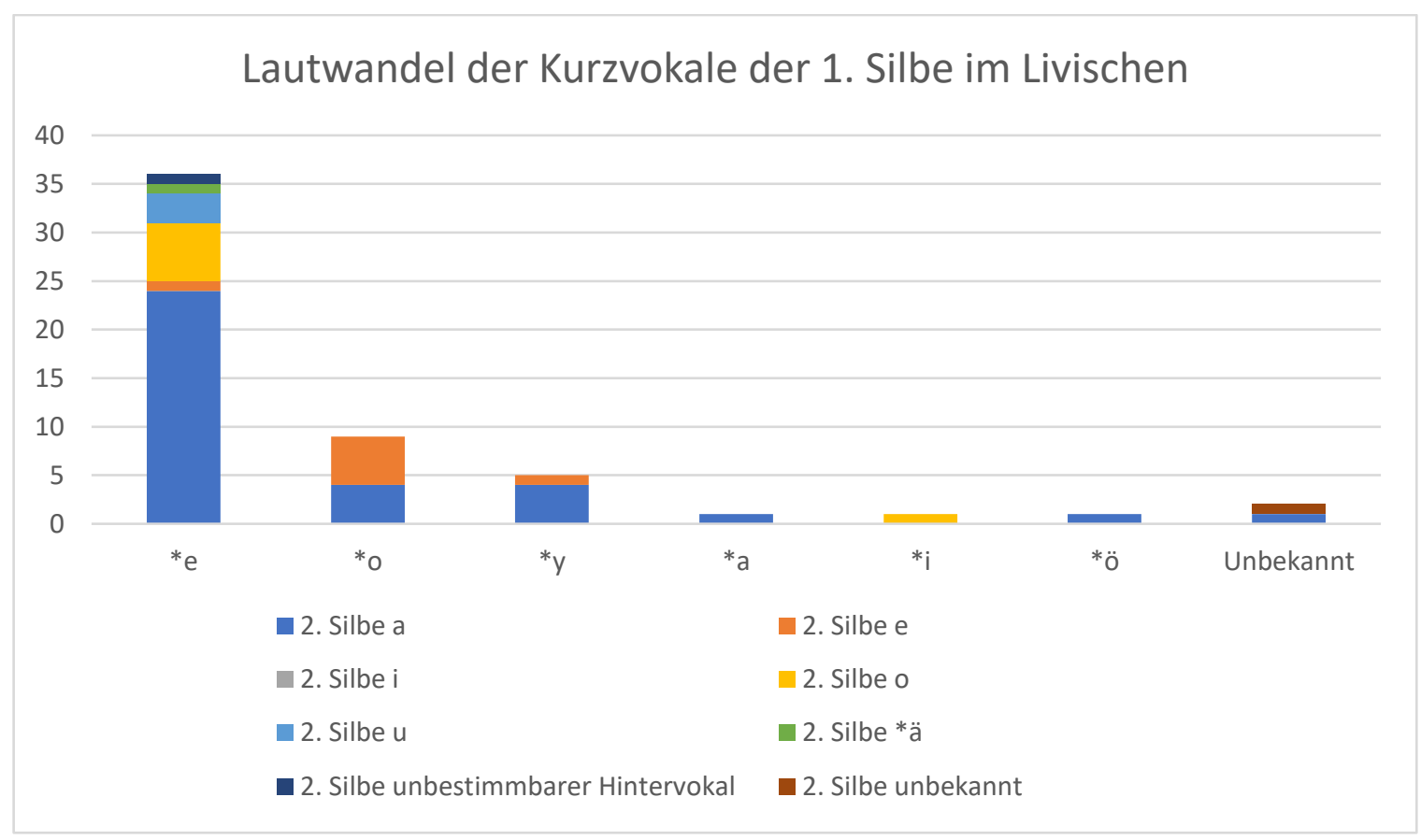

\subsubsection{Südestnisch}

Im Südestnischen lassen sich anhand der vorliegenden Belege insgesamt 99 für die Entwicklungen der ersten Silbe als einschlägig identifizieren. Auf * $e$ gehen in der ersten Silbe 42 Belege zurück, auf $* o$ sogar 53. Dem * $u$ der ersten Silbe lassen sich zwei Belege zuordnen. Die Vertretung von $* a$ umfasst einen Beleg, die historische Vertretung eines Belegs kann nicht bestimmt werden. Im Südestnischen liegt der gleiche Trend vor, der sich bereits bei den anderen Sprachen abgezeichnet hat. Während die Laute * $e$ und * $o$ das Zentrum der Entwicklungen bilden, liegen für die Lautwandel weiterer Vokale nur wenige Belege vor. Unter Berücksichtigung ihrer Folgesilben lassen sich die Belege gemäß der nachfolgenden Abbildung darstellen. Nicht einschlägige Belege des Korpus wurden auch hier nicht berücksichtigt. 


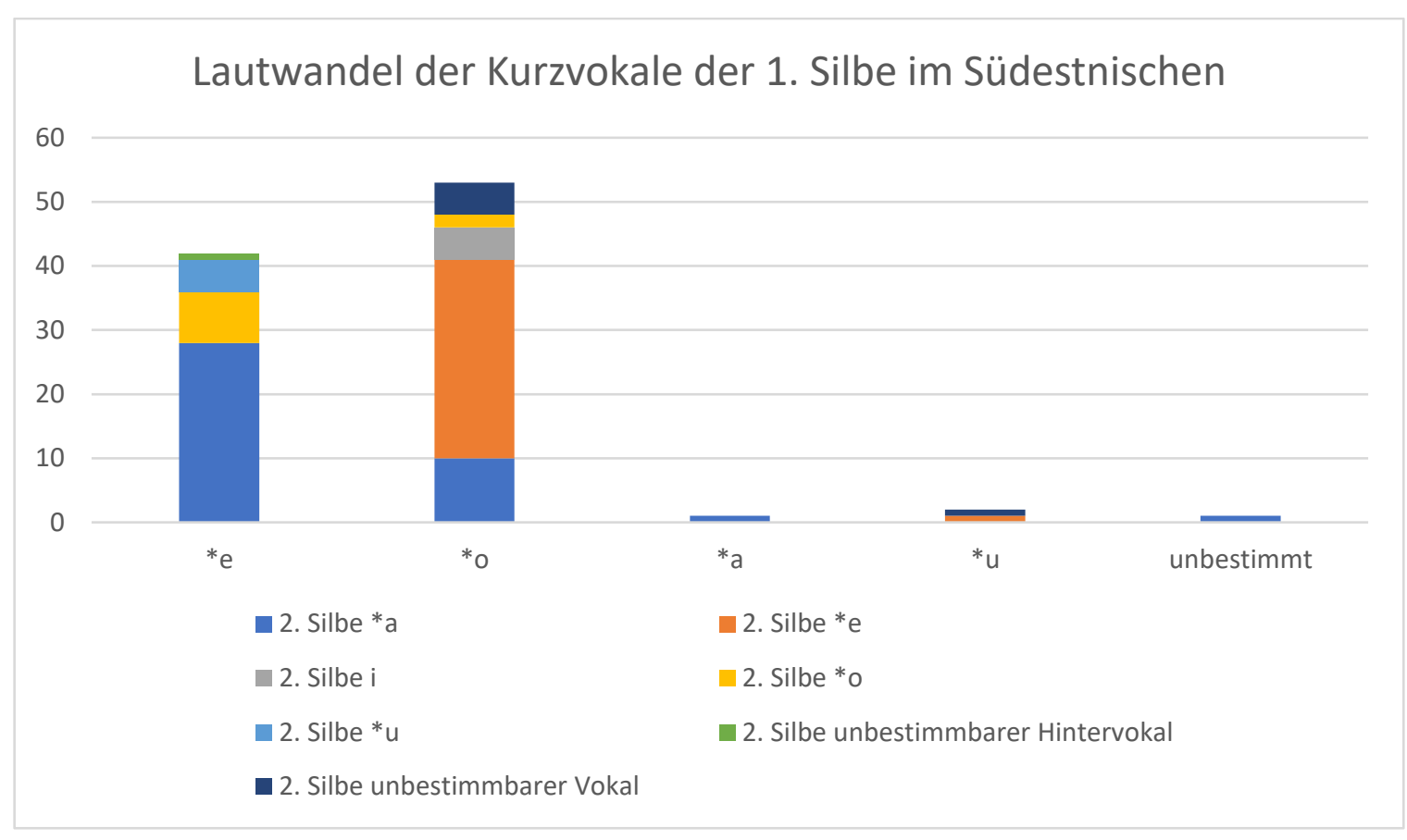

5.3.2 $* e>\tilde{o}$

Zunächst steht die Diskussion der Belege aus, die auf ein ursprüngliches ${ }^{*} e$ in der ersten Silbe zurückgehen. Sie machen in dieser Untersuchung die größte Gruppierung aus.

\subsubsection{Estnisch}

Betrachtet man das Estnische, so zeigt sich für die Entwicklung $* e>\tilde{o}$ in der ersten Silbe die nachfolgende Darstellung im Hinblick auf die zweite Silbe. Belege, die in nichtersten Silben im Estnischen einen Lautwandel $* o>u$ vollzogen haben (EKÄ 155), werden der historischen Vertretung in Form des Vokals $o$ zugeordnet.

$\begin{array}{cr}\text { Vokal der zweiten Silbe } & \text { Anzahl } \\ a & 32 \\ o & 12 \\ u & 6\end{array}$

Unbestimmbarer

Hintervokal 1 
Die zugehörigen Belege lassen sich folgendermaßen aufschlüsseln:

mit zweiter Silbe, die auf *a zurückgeht:

(2) est. hôlm, gen. - $a$ 'Rockschoß',

(74) est. ôhtu 'Abend, Westen',

(4) est. hõra- (in Zuss. mit hõrasilm 'starres

(98) est. põd|er, gen. -ra 'Elch, Elentier',

Auge'),

(106) est. põ̃sas 'Busch',

(5) est. hõrak- (in Zuss.) 'Johannisbeere',

(107) est. põrand 'Fußboden',

(14) est. kõla 'Gerät zum Weben eines Gür-

(109) est. põrn, gen. - $a$ 'Milz',

tels',

(120) est. sõb|er, gen. -ra 'Freund',

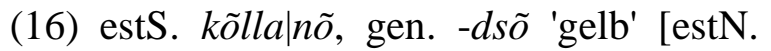

(122) est. sõel, gen. - $a$ 'Sieb',

kold, gen. kolla 'Bärlapp (eine gelbe Farbe

(133) est. sõsar 'Schwester', gebende Pflanze); Dotter, Eigelb', kollane 'gelb']

(134) est. sõstar 'Johannisbeere',

(18) est. kõlvata 'taugen',

(138) est. tõbras 'Vieh'

(26) estS. kõrd, gen. kõrra 'Ordnung, Reihe'

(141) est. tõmmata 'ziehen', [estN. kord id.],

(148) est. tõrv, gen. - a 'Teer', (75) est. rõngas

'Ring, Reifen',

(33) estS. kõ|tar', gen. -dara 'Speiche' [estN.

kodar id.],

(152) est. võhk, gen. võha 'Pflanze aus der Fa-

(41) est. lõkatada 'aufflammen',

(48) est. mõla 'Ruder',

(53) est. mõr|d, gen. -ra 'Fischreuse',

(59) est. nod $\mid$ er, gen. -ra 'schwach, schwach-

köpfig',

milie der Araceae',

(153) est. võl $\mid g$, gen. - $a$ 'Schuld',

(156) est. võr|d, gen. -ra '[in Zuss.] gleich;

Wert, Betrag',

(158) est. ? võrkida (Wied.) 'ermatten, erschöpfen',

(60) est. nõel, gen. - $a$ 'Nadel',

(160) est. võsa 'Schössling, Spross',

(65) est. nor $\mid k$, gen. - $g a$ 'schwach',

mit zweiter Silbe, die auf $* o$ zurückgeht:

(3) est. hõl $\mid p$, gen. - bu 'Leichtigkeit',

(147) est. tõru 'Eichel',

(37) est. lõbu 'Vergnügen',

(154) est. võlu 'Zauber',

(52) est. mõnu 'Genuss [SSA auch: Lärm]',

(155) est. võnnu- (in Zusammensetzungen)

(90) est. mõtus, gen. -e (dial.) 'Auerhuhn, - 'völlig fremd',

hahn',

(157) est. võr $\mid k$, gen. - gu 'Netz',

(58) est. nõbu 'Cousin',

(159) est. võrsuda 'sprießen';

(102) est. põl|d, gen. -lu 'Feld', 
(115) est. rõht, gen. rõhu 'waagerecht seiend

(Holz in der Wand, Stange im Zaun)',

mit zweiter Silbe, die auf * $u$ zurückgeht:

(6) est. $h \tilde{o r} \mid k$, gen. -gu 'appetitlich',

(70) est. $\tilde{o} k$, gen. $\tilde{o} h u$ 'Luft',

(40) est. lõhmus 'Linde',

(100) est. põhk, gen. - $u$ 'Kurzstroh, Streu',

(44) est. mõdu 'Met',

(108) est. põrm, gen. - $u$ 'Staub, Asche';

mit unbestimmbarem Hintervokal:

(55) est. mõtelda 'denken, nachdenken'.

Aus dieser Darstellung lässt sich zunächst schließen, dass der Lautwandel * $e>\tilde{o}$ im Estnischen immer dann einzutreten scheint, wenn die darauffolgende Silbe hintervokalisch ist oder auf einen ebensolchen Vokal als historische Vertretung zurückgeführt werden kann. Belege, deren zweite Silbe historisch vordervokalisch ist, treten nicht auf.

\section{ANMERKUNGEN}

$(\mathbf{1 6}, \mathbf{2 6}, 33)$ Die südestnischen Formen der Belege sind wohl als ursprüngliche Vertretung ansetzbar. Für das Nordestnische ist in der ersten Silbe hier eine Entwicklung $* e>\tilde{o}>o$ festzuhalten, im Südestnischen ist dagegen nur die erste Stufe des Lautwandels vollzogen worden (vgl. UnIVERE 1986: 69; KeEM 2002: 33). Die These wird durch die Überlegungen von WIIK (1986: 41) gedeckt, der eine derartige Lautentwicklung ebenfalls annimmt.

(55) In LÄGLOS (II: 281) wird ein Stamm urfi. *mettV-rekonstruiert. Zwei Gründe sprechen dafür, dass der Vokal der zweiten Silbe dieses Stamms ein Hintervokal gewesen sein muss: 1) Der Lautwandel $* e>\tilde{o}$ scheint nur bei Wörtern einzutreten, deren zweite Silbe hintervokalisch ist. 2) Es wird von einem germanischen Lehnwort ausgegangen, dessen zweite Silbe hintervokalisch gewesen sei (vgl. urgerm *metō(ja)n-, *metan- (ebd.)). Ein Hintervokal muss folglich in einer früheren Wortform vorgelegen haben, er ist jedoch nicht näher bestimmbar.

An den Stamm des Wortes trat später ein Ableitungssuffix le- (KASIK 2015: 160ff.). Laut LAANEST (1982: 274) ist der Bestandteil $l$ in frequentativen Ableitungssuffixen aller uralischen Sprachen bekannt. Dass die Ableitung mit le- in diesem Wort wohl eine jüngere Entwicklung ist, zeigt sich auch daran, dass nicht in allen ostseefinnischen Sprachen eine Entsprechung vorliegt: Es gibt sie lediglich noch im Wotischen, Livischen (EES 293) und Südestnischen. Der 
Ableitungstyp ist im Estnischen auch heute noch produktiv, da weiterhin neue Verben gebildet werden, z.B. est. aspektlema 'vor sich hin aspektieren' (KASIK 2015: 161).

(58) Von EES (323) wird eine alte Ableitung $-u$ angesetzt. Nach der Form des ins Ostseefinnische entlehnten baltischen Wortes zu schließen, ist eine Entwicklung von $* o>u$ in der zweiten Silbe ebenso zu erwägen, vgl. urlit. nepotis, nepuotis, nepatis 'Enkel; Sohn des Bruders oder der Schwester, Sohn des Cousins/der Cousine' (SSA II: 213; EES 323).

$(60,122)$ Vor $l$ wird der Klusil $* k$ im Silbenauslaut vokalisiert (EKÄ 86), vgl. zu (60) urfi. *nekla /*nēkla (LÄGLOS II: 297) und zu (122) urfi. *sekla / *sēkla (LÄGLOS III: 229).

(74) Die historische Vertretung der zweiten Silbe lautet wohl $a$, vgl. auch fi. ehta, ehtä 'abendliche oder nächtliche Gemeinschaftsarbeit; Abend' (SKES I: 35; SSA I: 101) wot. õhtago 'Abend, Westen' liv. $\overline{\tilde{o}}^{\prime} d \tilde{o} g$ id. ${ }^{51} \sim$ estS. õdak $\sim \tilde{o t a k}$ id.

(106) Eine Vokalisierung des $* n$ der Verbindung $* n s$ tritt ein, also $*^{*} n s>V s$ (EKÄ 104). HoLST (2001: 93f.) schlägt zwei Optionen vor: 1) Der Konsonant sei zuerst vokalisiert worden, ehe sich der Vokal der ersten Silbe zu $\widetilde{o}$ wandeln konnte, oder 2) vor Eintritt der Vokalisierung sei es zum Lautwandel * $e>\tilde{o}$ gekommen. Die Reihenfolge sei seiner Meinung nach für den tatsächlichen Ablauf unerheblich, denn beide Optionen würden in der heutigen Wortform zum gleichen Ergebnis führen. Da man es bei der Vokalisierung von $* n$ mit einer Entwicklung zu tun hat, die laut LAANEST (1982: 124f.) „konsequent in den Sprachen der Südwestgruppe des Ostseefinnischen“" stattgefunden hat, ist zu erwägen, ob der Lautwandel ${ }^{*} n s>V s$ älter ist als die Entstehung von o, vgl. dazu auch die wotischen und livischen Entsprechungen in Kapitel 5.3.2.2 und 5.3.2.3. Aufgrund der geringen Anzahl an Belegen kann diese Überlegung jedoch nicht gesichert werden. Eine parallele Entwicklung in den Einzelsprachen ist ebenso möglich.

(133) Dem $i$ der finnischen Entsprechung fi. sisar 'Schwester' scheint eine andere Entwicklung zugrunde zu liegen. HOLST (2001: 92) führt an, dass es im Baltischen in dieser Position vor Plosiv oder Sibilant eine wechselnde Vertretung von $i$ und $e$ gebe, sodass diese Zweigeteiltheit ein möglicher Grund für die Unterschiede sei: Das Finnische habe seiner Auffassung nach die

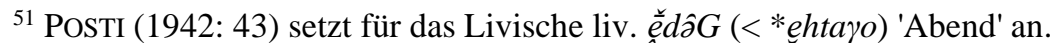


Form mit $* i$ in der ersten Silbe entlehnt, das Estnische die mit $* e$, in der es später zum Lautwandel $* e>\tilde{o}$ kam.

(134) Die ursprüngliche Form ist unbekannt, die finnische Entsprechung auffällig: fi. siestar 'schwarze Johannisbeere'. Ansetzbar ist wohl, dass man es mit einem historischen * $e$ zu tun hat und das Finnische in der Entwicklung dieses Wortes abweicht, während das Estnische den Lautwandel $* e>\tilde{o}$ vollzieht. Stützen lässt sich die Annahme von *e durch weitere Entsprechungen, vgl. wot. sõssar 'Johannisbeere', weps. sestrikäine 'rote Johannisbeere' und lüd. sestroi 'rote Johannisbeere, lokal auch schwarze Johannisbeere' (SSA III: 173f.).

(138) Angesetzt wird urfi. *tepras oder *teuras (LÄGLOS III: 291f.). Die Form *tepras ist wahrscheinlicher, da ansonsten eine Entwicklung $* u>b$ für das Estnische hätte angesetzt werden müssen. Eine Vokalisierung liegt bei diesem Beleg im Finnischen vor. ${ }^{52}$

(158) Eine unsichere Entsprechung liegt vor, die lediglich bei WIEDEMANN (1923: 1397) als dialektale Vertretung (Tartu) belegt ist. ${ }^{53}$ Das $i$ der zweiten Silbe ist ein Ableitungssuffix (KASIK 2015: 172f.), der Wortstamm ursprünglich wohl mit $a$ als hintervokalisch ansetzbar, wie die anderen ostseefinnischen Entsprechungen implizieren: fi. verkkainen 'langsam; gemächlich' liv. vẹrka 'langsam schleppend (z.B. Krankheit)'). Eine gesicherte abschließende Bewertung der etymologischen Entwicklung bleibt aufgrund des Mangels weiterführender Belege offen. EES behandelt dieses Wort nicht.

\section{GEGENPROBE}

Geht man nun anhand der soeben diskutierten Belege davon aus, dass es sich bei * $e>\tilde{o}$ um eine Entwicklung handelt, die dann eintritt, wenn der Vokal der Folgesilbe hintervokalisch ist, so stellt sich die Frage nach einem Gegenbeweis, der im Folgenden erbracht werden soll. Dazu

\footnotetext{
${ }^{52}$ Die Belege (98) und (120) gehören ebenso an diese Stelle, da sie einem ähnlichen Typus entsprechen. Die estnischen Belege sind regelmäßig und eine Vokalisierung liegt nicht vor. In den finnischen Entsprechungen ist diese dagegen anzutreffen, vgl. zu (98) fi. peura 'wildes Rentier, Waldrentier, Elch' und zu (120) fi. seura 'Gesellschaft, Zusammensein, Begleitung, Kreis von Menschen'. Der Beleg (59) gehört ebenfalls zu diesen Wörtern, die im Finnischen den Klusil im Silbenauslaut vokalisieren vgl. fi. nöyrä 'ergeben, unterwürfig'. Als ursprüngliche Form ist wohl *netra ansetzbar. Zur Vokalisierung der Klusile im Finnischen vgl. auch LAANEST (1982: 133).

${ }^{53}$ Das Wort wurde aufgrund seiner livischen Entsprechung, liv. vẹrka 'langsam schleppend (z.B. Krankheit)', in das Korpus aufgenommen. Zum Livischen vgl. Kapitel 5.3.2.3.
} 
wurden auf Grundlage der etymologischen Wörterbücher SSA und EES weitere Belege ermittelt, deren zweite Silbe in ihrer heutigen Form hintervokalisch ist, während * $e$ in der ersten Silbe bewahrt wird. Folgende Kriterien liegen der Zusammenstellung zugrunde: Es wurden nur Wörter berücksichtigt, deren erste Silbe *e lautet. Sie müssen in einen nachweisbaren etymologischen Zusammenhang mit anderen ostseefinnischen Sprachen gebracht werden können. Nicht beachtet wurden Ableitungen von Wörtern, deren Wortstamm bekannt ist, Wörter mit unsicherer Etymologie sowie Entlehnungen, die eindeutig jüngeren Alters sind und über einen alten Lautstand wenig aussagen, z.B. aus dem Niederdeutschen oder dem Russischen. Ebenso wenig aufgenommen wurden künstlich gebildete Formen oder Varianten. Aus dem Korpus waren an dieser Stelle keine Belege zu ergänzen.

$1 \quad$ est. edasi 'weiter, vorwärts, ferner'

fi. et $\ddot{a}$ - 'fern'

fi.-wolg. ečkä oder ečä + Kasussuffix 'weit, fern' (UEW II: 624)

SSA (I: 109f.), (EES 57)

2 est. elada 'existieren, leben, wohnen'

fi. elää 'leben, wohnen'

ural. elä- 'leben' (UEW I: 73)

SSA (I: 103f.), EES (60)

3 est. ema 'Mutter'

fi. emä 'Muttertier; Tierweibchen; sehr groß, ausgewachsen; Haupt; Gebärmutter' ural. emä 'Mutter, Weib' (UEW I: 74)

SSA (I: 104), EES (60f.)

4 est. enam 'mehr'

fi. enä 'groß; viel'

ural. enä 'groß viel' (UEW I: 74)

SSA (I 106), EES (61)

5 est. era- 'privat-'

fi. erä 'getrenntes Stück von etwas; (An)teil; Partie; Mal'

SSA (I: 107) EES (62)

6 est. hell, gen. hella 'zart, liebevoll, sanft'

fi. hellä 'empfindlich, wund; zärtlich, locker, mild (Wetter)'

SSA (I: 153f.), EES (74) 
est. kebja 'flink, hurtig, leichtfüßig; leicht (Gewicht)'

fi. kepeä 'leicht'

fiugr. (? ural.) kepä 'leicht' (UEW I: 146)

SSA (I: 344), EES (139)

8

est. keder, gen. kedra 'Scheibe, Drehscheibe'

fi. kehrä 'Spinnwirtel; Ring'

fi.-wolg. kečrä ( > *kešträ) 'Spindel; ? spinnen' (UEW II: 656)

SSA (I: 336), EES (139f.)

9 est. keha 'Körper; Rumpf'

fi. kehä 'Kreis, Radkranz; [...]'

fiugr. kečä 'Kreis, Ring, Reifen' (UEW I: 141)

SSA (I: 337), EES (141)

10 est. kehata 'sich trauen, den Mut haben, wagen; übers Herz bringen'

fi. kehdata 'sich nicht schämen, wagen; nicht die Mühe scheuen, Lust haben'

SSA (I: 335), ? est. dial. kõhata 'können; sich schämen, ekeln', welches in EES nicht behandelt wird (EES 142)

11 est. kehv, gen. kehva 'ärmlich, dürftig, gering, schlecht, unbefriedigend'

fi. $k \ddot{o y} h \ddot{a}$ 'arm'

urfi. *keühä (LÄGLOS II: 143)

SSA (I: 486), EES (142); ? germ. Lehnwort (LÄGLOS II: 143)

12 est. kelk, gen. kelgu 'Schlitten'

fi. kelkka 'Schlitten'

*kelkka (LÄGLOS II: 76f.)

SSA (I: 340), EES (143); germ. oder jüngeres Lehnwort LÄGLOS (II: 76f.)

13 est. kell, gen. kella 'Uhr, Glocke'

fi. kello 'Glocke, Schelle, Uhr'

urfi. *kello (LÄGLOS II: 78)

SSA (I: 340), EES (143); germ. Lehnwort (LÄGLOS II: 78)

14 est. kelp, gen. kelba 'Walm'

urfi. *kelppä / *kelppa (LÄGLOS II: 79)

EES (143); kaum germ. Lehnwort (LÄGLOS II: 79)

15 est. kenus 'stolz nach hinten gebogen'

fi. keno 'Bootsheck od. -bug; hochgebogenes Vorderteil des Schlittens' 
urfi. *keno (LÄGLOS II: 82)

SSA (I: 343), EES (144); germ. Lehnwort (LÄGLOS II: 82f.)

16 est. kepsata 'leicht hüpfen, mit kleinen Schritten leicht schreiten', keps, gen. - $i,-u$ 'Hüpfer'

fi. kepsa 'flink'

SSA (I: 344), EES (144)

17 est. kera 'Knäuel'

fi. kerä 'Knäuel'

SSA (I 348), EES (144)

18 est. kerjata 'betteln'

fi. kerjätä 'betteln'

fiugr. kerä- 'bitten' (UEW I: 149)

SSA (I 346), EES (145)

19 est. kesa 'Brache, Brachfeld'

fi. kesä 'Sommer'

fi.-wolg. kesä 'Sommer' (UEW II: 660f.)

SSA (I: 351), EES (146)

20 est. kesta 'dauern, währen'

fi. kestää '(aus)halten, vertragen; dauern'

SSA (I: 350), laut EES (146) zu est. kesk 'mitten'

21 est. kevad(e) 'Frühling'

fi. kevät 'Frühling'

fi.-wolg. keøä 'Name einer milden Jahreszeit; Frühling, Sommer' (UEW II: 659)

SSA (I: 352), (EES 147)

22 est. lebada 'liegen'

fi. levätä '(aus)ruhen, rasten'

urfi. *lepä- (LÄGLOS II: 198)

SSA (II: 67f.), EES (232); ? germ. Lehnwort (LÄGLOS II: 198f.)

23 est. lehm, gen. lehma 'Kuh'

fi. lehmä 'Kuh'

fi.-wolg. lešmä 'irgendein größeres Haustier: Kuh, Pferd' (UEW II: 689)

SSA (II: 58), EES (234) 
24 est. lennata 'fliegen, schnell fortbewegen'

fi. lentää 'fliegen'

SSA (II: 64), EES (236)

25 est. lepp, gen. lepa 'Erle (Alnus)'

fi. leppä 'Erle (Alnus); Blut'

fi.-wolg. leppä 'Erle' (UEW II: 689)

SSA (II: 64f.), EES (237)

26 est. lest, gen. lesta 'Flunder (Platichthys flesus)' $\sim$ liv. liestā 'Flunder' EES (237)

27 est. neli, gen. nelja 'vier'

fi. neljä 'vier'

fiugr. ńeljä (neljä) 'vier' (UEW I: 315f.)

SSA (II: 212), EES (310)

28 est. pedajas 'Kiefer, Föhre'

fi. petäjä 'Kiefer, Föhre'

fi.-perm. pečä penčä 'Kiefer, Föhre; Pinus sylvestris' (UEW II: 727)

SSA (II: 345f.), EES (358)

29 est. peksta 'schlagen, prügeln, peitschen'

fi. piestä 'prügeln, peitschen, buttern [...]'

ural. peksä- 'schlagen' (UEW I: 368)

SSA (II: 349), (EES 360)

30 est. peljata 'scheuen, (sich) fürchten'

fi. pelätä '(sich) fürchten'

ural. pele- 'fürchten, sich fürchten' (UEW I: 370)

SSA (II: 335), EES (361)

31 est. рери 'Hintern'

fi. ugs. рерри 'Hintern'

SSA (II: 338, EES (362)

32 est. pesa 'Nest'

fi. pesä 'Tierbehausung (Bau, Nest'); Hausstand [...]

SSA (II: 343f.), EES (363)

33 est. pesta 'waschen'

fi. pestä 'waschen' 
fi.-wolg. pese- 'waschen (den Kopf?) (UEW II: 729)

SSA (II: 343), EES (363)

34 est. petta 'betrügen, fälschen'

fi. pettää 'betrügen'

SSA (II: 345), EES (363)

35 est. plehku 'lauf-, flucht-' wot. plehkuu id.

EES (375)

36 est. rebane 'Fuchs'

fi. repo 'Fuchs'

osfi. (urfi.) *repäinen (SSA III: 65)

fiugr. repä ( -ćs) 'Fuchs' (UEW I: 423)

SSA (III: 65f.), EES (421f.)

37 est. segada 'mischen, vermischen [...]'

fi. seka 'etw. Gemischtes', sekoittaa 'mischen, vermischen'

SSA (III: 166), EES (465)

38 est. selg, gen. selja 'Rücken'

fi. selkä 'Rücken'

fi.-wolg. śelkä 'Rücken' (UEW II: 772)

SSA (III: 167), EES (466)

39 est. sepp, gen. sepa 'Schmied'

fi. seppä 'Schmied'

fiugr. śeppä 'geschickt' (UEW I: 474)

SSA (III: 169), EES (467f.)

40 est. Wied. seba 'das nach oben gebogene Ende des Schlittens'

fiugr. śepä 'Hals, Nacken' (UEW I: 473)

fi. sepä 'das nach oben gebogene Vorderende (bzw. ein Teil dess.) des Pferdeschlittens'

SSA (III: 169), in EES nicht behandelt

41 est. serv, gen. serva 'Rand, Saum'

fi. särvi 'Rand, Seite, Kante'

SSA (III: 242), EES (468)

42 est. teha 'tun, machen'

fi. tehdä 'tun, machen' 
fiugr. teke- 'tun, machen' (UEW I: 519)

SSA (III: 279), EES (520f.)

est. tela 'Rundholz zum Transport von Booten'

fi. tela 'Rundholz als Unterlage (eines Holzstapels usw.) oder als Rolle beim Transport (eines Bootes über Land o.ä.); Walze; nicht verbrannter, angekohlter Baumstamm auf der Schwende' urfi. * tela (LÄGLOS III: 284)

SSA (III: 280f.), EES (522); VMS (II: 497); germ. Lehnwort (LÄGLOS III: 284)

$44 \quad$ est. tema Pronomen-3Sg

fi. tämä 'diese/r/s'

ural. $t \ddot{a}(\sim t e \sim t i)$ 'dieser' (UEW I: 513)

SSA (III: 355), EES (523)

45 est. tera 'Schneide, Klinge'

fi. terä 'Klinge, Schneide, Sägeblatt, Bohrer'

fiugr. terä 'Schneide, Spitze' (UEW I: 522)

SSA (III: 287), EES (524f.)

Zur Gegenprobe lassen sich folgende Anmerkungen festhalten:

1-9, 17-25, 27-30, 32-34, 36, 38-42, 45: Der Abbau der Vokalharmonie ist für die Abweichung im Estnischen ursächlich. Es ist von historisch vordervokalischen zweiten Silben auszugehen. 10: Die Entwicklung ist unklar, die Variante mit õ sei lediglich eine ungesicherte dialektale Vertretung, welche in EES nicht behandelt wird.

11: Der Ausgangslaut der ersten Silbe ist ein Diphthong, dessen zweiter Bestandteil der Metathese $* V h>h V$ unterliegt (EKÄ 142). Obschon hier keine tatsächliche Vertretung eines historisch kurzen Vokals vorliegt, kann als Grund für das Bewahren von * $e$ der Abbau der Vokalharmonie genannt werden.

12: Warum im Estnischen kein Lautwandel eintritt, ist nicht endgültig erklärbar. Ein möglicher Ansatz ist die in LÄGLOS (II: 76f.) angeführte wiederholte Entlehnung, sodass das Wort womöglich erst nach der Entstehung von $\tilde{o}$ aufgekommen ist.

13, 15, 16, 35, 37: Ungeklärt ist auch hier, warum der Lautwandel nicht vorliegt.

14: In LÄGLOS (II: 79) werden zwei mögliche Stämme angesetzt. Der Stamm mit vordervokalischem Auslaut entspricht der Entwicklung der Belege, die die Vokalharmonie abgebaut haben. 
26: Eine balt. Entlehnung wird in EES (237) angesetzt, deren Einfluss womöglich als Ursache für die Abweichung zugrunde gelegt werden kann, vgl. lit. lekštas 'flach, platt; sanft geneigt, mild, schräg, schief', lett. lesns 'flach, platt', lēsns id., lēss id., leste 'Scholle; Flunder'.

31: Laut etymologischer Wörterbücher seien es Formen aus der Kindersprache, die für die Abweichung wohl ursächlich sind, vgl. fi. perse 'Arsch' bzw. est. perse 'Hintern, Arsch' (SSA II: 338, EES 362).

43: Die estnische Entsprechung sei nur in den Küstendialekten vertreten und < fi. (vgl. VMS II: 497).

44: Für das Estnische wird in UEW (I: 514) ein Stammvokal *e angesetzt. Die Gebrauchshäufigkeit des Pronomens ist wohl als Grund für dessen Bewahren ansetzbar.

Nach der vorausgegangenen Betrachtung der Gegenbelege zu schließen, ist der häufigste Grund für abweichende Formen im Estnischen der Abbau der Vokalharmonie. Sie fügen sich in die zuvor formulierte Annahme eines Lautwandels bei Hintervokal in der Folgesilbe nahtlos ein. Das Zahlenverhältnis stützt diese Hypothese ebenso. Die abweichenden Belege, für die kein Lösungsansatz gefunden wurde, liegen im Vergleich zu den Belegen mit Lautwandel in der ersten Silbe nur in geringer Anzahl vor. Von insgesamt 45 Belegen der Gegenprobe treten lediglich in fünf Fällen Unklarheiten auf, für welche mangels weiterführender Angaben keine Antwort gefunden werden konnte. Sie können als erwartbarer Grad an Abweichungen einzelsprachlicher Entwicklungen festgehalten werden.

Der Lautwandel der Belege, die in der ersten Silbe auf *e zurückgehen, ist auch in der Wissenschaft v.a. für das Estnische bereits vielfach beschrieben und wird von den Vertretern des $\tilde{o}$ als sekundäre Entwicklung als sicher angesehen. Die Thesen decken folglich die für das Estnische ermittelten Resultate (vgl. z.B. WIIK (1986: 40) und HOLST (2001: 78)).

\subsubsection{Wotisch}

Bei der Betrachtung des Lautwandels * $e>$ o im Wotischen zeigt sich, dass ebenfalls alle Belege dieser Entwicklung einem Hintervokal in der Folgesilbe zugeordnet werden können. Zur Veranschaulichung der Verteilung der Vokale der zweiten Silbe dient die nachfolgende Tabelle: 


$\begin{array}{cc}\text { Vokal der zweiten Silbe } & \text { Anzahl } \\ a & 26 \\ o & 2 \\ u & 3 \\ \text { Unbestimmbarer Hintervokal } & 1\end{array}$

$\mathrm{Zu}$ diesen Daten gehören die nachfolgend angeführten Belege: mit zweiter Silbe, die auf * $a$ zurückgeht:

(2) wot. ôlma 'Rockschoß',

(107) wot. põrmata 'Boden',

(4) wot. orahtaa 'vor dem Melken Milch ins (109) wot. põrna 'Milz',

Euter sammeln',

(117) wot. rõygaz 'Ring, Reifen',

(16) wot. kõlta 'gelb',

(120) wot. sõbra 'Freund',

(18) wot. kõlvata 'taugen',

(133) wot. sõzar 'Schwester',

(26) wot. kõrta 'Ordnung, Reihe',

(134) wot. sõssar 'Johannisbeere',

(33) wot. kõtara 'Speiche',

(138) wot. tõbraz 'Rentier, Elch',

(48) wot. mõla 'Ruder',

(141) wot. tõmmata 'ziehen',

(53) wot. mõrta 'Fischreuse',

(148) wot. tõrva 'Teer',

(59) wot. nõdra 'schwach, schwachköpfig',

(152) wot. võhka 'Calla palustris',

(65) wot. nõrkka 'düster, finster, missmutig, wehmütig',

(153) wot. võlka 'Schuld',

(74) wot. ôhtago 'Abend, Westen',

(156) wot. (sev)võrta 'Größe, mit der etw.

(98) wot. põdra 'Elch', bzgl. Menge, Wert usw. verglichen wird,

(106) wot. põ̃zaz 'Busch', ebenbürtig (alt), soviel',

(160) wot. võso 'Schössling, Spross';

mit zweiter Silbe, die auf $* u$ zurückgeht:

(44) wot. mõtu 'Met, Bier', (108) wot. põrmu 'Staub, Asche';

(100) wot. põhku 'Kurzstroh, Streu', mit zweiter Silbe, die auf $* o$ zurückgeht:

(102) wot. põlto 'Feld, Ackerstreifen', (157) wot. võrkko 'Netz';

mit unbestimmbarem Hintervokal:

(55) wot. mõtõlla 'denken, nachdenken'.

Die Darlegung der Daten stimmt mit den Verhältnissen im Estnischen überein: Es ist erkennbar, dass der Lautwandel $* e>\tilde{o}$ anscheinend auch im Wotischen immer dann eintritt, wenn die 
darauffolgende Silbe hintervokalisch ist. Abweichungen davon zeigen sich anhand der Belege nicht.

\section{ANMERKUNGEN}

(55) Das $\tilde{o}$ der zweiten Silbe ist zunächst als eine sekundäre Entwicklung festzuhalten, die auf *e zurückgeht. Jenes ist Teil einer Ableitung (-ele-/-ele-, vgl. ARISTE 1968: 118). Hinzuweisen ist auf den rekonstruierten Stamm urfi. *mettV- (LÄGLOS II: 281), für den bereits bei der Diskussion der estnischen Entsprechung ein Hintervokal angesetzt wurde, vgl. Kapitel 5.3.2.1. Es ist ebenso anzunehmen, dass das Ableitungssuffix zu einem späteren Zeitpunkt an den Stamm getreten ist und die historische Vertretung der zweiten Silbe den Lautwandel zuvor ausgelöst hat.

(106) Eine Vokalisierung * $n s>V s$ tritt ein (VKÄ 92f.). Wie schon bei der estnischen Entsprechung dieses Belegs bleibt offen, in welcher Reihenfolge die Vokalisierung von $* n$ und die Entwicklung $* e>\tilde{o}$ abgelaufen sind; vgl. Kapitel 5.3.2.1.

(133) Vgl. die Erläuterungen zur estnischen Entsprechung in Kapitel 5.3.2.1

(134) Vgl. die Erläuterungen zur estnischen Entsprechung in Kapitel 5.3.2.1

(160) - $o$ ist ein Ableitungssuffix (ARISTE 1968: 115). Der Beleg geht wohl in der zweiten Silbe auf *a zurück (vgl. est. võsa 'Schössling, Spross' liv. võza 'Trieb, Sprössling, Schössling' fi. vesa 'Wurzel- Stocktrieb, Spross, Schoss, Sprößling').

\section{ABWEICHUNGEN IN DER ERSTEN SILBE}

(60) wot. nigla 'Nadel' (147) wot. turu 'Eichel' (122) wot. sigla 'Sieb'

$(60,122)$ Karelischer Einfluss bewirkte wohl einen Lautwandel $* e>i$ (VKÄ 126). (147) SSA (III: 285) setzt eine unsichere Entlehnung aus einem estnischen Dialekt an. So gibt es eine Form est. turu in einigen Dialekten in Nordost- und Ostestland (Väike-Maarja, Kadrina, Viru-Jaagupi, Palamuse) (VMS II: 564). Vor dem Hintergrund der geographischen Verteilung 
der Dialekte und ihrer relativen Nähe zum wotischen Sprachgebiet scheint diese Erklärung plausibel.

\section{GEGENPROBE}

Auch für das Wotische wurden auf Grundlage von SSA und EES Wörter ermittelt, die einer Gegenprobe zu den hier dargelegten Belegen dienen sollen. Die Kriterien sind dabei die gleichen wie für das Estnische $(* e$ in der ersten Silbe bewahrt, etymologisch nachweisbar, keine Ableitung oder jüngere Entlehnung). Zusammengestellt werden konnten die nachfolgenden Belege:

$1 \quad$ wot. elkkā 'in hohen Tönen klingen'

fi. helkkyä 'in hohen Tönen klingen; schwappen'

SSA (I: 153), EES (73f.)

$2 \quad$ wot. (Tsv.) kelk; (Kukk) kelkka 'Schlitten'

fi. kelkka 'Schlitten'

*kelkka (LÄGLOS II: 76f.)

SSA (I: 340), EES (143); germ. oder jüngeres Lehnwort (LÄGLOS II: 76f.)

3 wot. tšellä 'Glocke; Glockenblume', kello (Kukk,) id., tšello 'Uhr'

fi. kello 'Glocke, Schelle, Uhr'

urfi. *kello (LÄGLOS II: 78)

SSA (I: 340), EES (143); germ. Lehnwort (LÄGLOS II: 78)

$4 \quad$ wot. lello id.

fi. lelu 'Spielzeug'

SSA (II: 62), EES (236)

5 wot. (Kukk) pellovaz, 'Lein, Flachs'

fi. pellava 'Lein, Flachs'

SSA (II: 333)

6 wot. plehkuu 'lauf-, flucht-' est. plehku id.

EES (375)

7 wot. repo 'Fuchs'

fi. repo 'Fuchs'

osfi. (urfi.) *repäinen (SSA III: 65)

fiugr. repä ( -ćs) 'Fuchs' (UEW I: 423)

SSA (III: 65f.), EES (421f.) 
1: Laut EES (73f.) sei es eine veraltete Form, weitere Begründungen für eine Abweichung konnten nicht festgestellt werden.

2: Die Form wot. kelkka stammt aus dem Dialekt Kukkuzi, für welchen charakteristisch ist, dass der Laut $\tilde{o}$ nicht auftritt (VKÄ 126). Die Form wot. kelk ist nach TsVETKOV notiert und gibt somit keine Auskunft über die Entwicklung, da dieser einen Teil des wotischen Wortschatzes neu geschaffen hat (vgl. ERNITS 2009: 105).

3: Die Form wot. ť̌ellä 'Glocke; Glockenblume' verhält sich erwartungsgemäß. Für wot. kello id. wird der Dialekt Kukkuzi angesetzt, zudem < fi. Unklar bleibt die Entwicklung von wot. tšello 'Uhr', von Fremdeinfluss ist wohl ebenfalls auszugehen.

4: Die Entwicklung ist unklar, auch hier ist Fremdeinfluss wohl ansetzbar (vgl. est. lelu 'Spielzeug' < fi. (EES 236)).

5: Vertretung aus dem Dialekt Kukkuzi, s. auch 2.

6: Unklar ist, warum der Lautwandel nicht eintritt. Das wotische Wort tritt lediglich im Dialekt Jõgõperä auf, kann also eine dialektale Abweichung sein.

7: wot. -o ist Ableitungssuffix (vgl. ARISTE 1968: 115), der Wortstamm ursprünglich vordervokalisch.

8: Zwei Dinge werden angesetzt: Das Wort stammt aus dem Dialekt Kukkuzi (vgl. auch 2) und sei $<$ ing.

Ferner sind anhand des Korpus die nachfolgenden Belege zu dieser Gegenprobe zu ergänzen:

3 wot. helppo 'leicht, mühelos'

fi. helppo 'leicht; locker'

urfi. *helppo (LÄGLOS I: 93)

6 wot. erkku 'Delikatesse'

fi. herkku 'Delikatesse, Leibgericht'

41 wot. lekko 'Flamme'

fi. lekko 'Flamme, Feuer; Hitze, Sonnenglut' 
52 wot. meno 'Gang'

fi. meno 'Gehen, Gang, Fahrt, (Ver)lauf; Radau'

155 wot. ventõizõ̃ 'völlig fremd'

fi. vento 'völlig fremd'

urfi. *vento (LÄGLOS III: 387)

$(6,41)$ SSA (I: 154; II: 61) setzt den Dialekt Kukkuzi für diese Formen an.

$(3,52,155)$ Fremdeinfluss scheint hier möglich zu sein, kann jedoch nicht sicher nachgewiesen werden. ${ }^{54} \mathrm{Zu}$ (52) vgl. z.B. ing. helppo 'mühelos, leicht; locker' (IMS 56)

Im Wotischen ist die Gegenprobe weniger umfangreich als im Estnischen, was auch aus der vorhandenen Vokalharmonie zu erklären ist. Es liegen stattdessen andere Kriterien für die Abweichungen der ersten Silbe als im Estnischen zugrunde, wie beispielsweise Formen aus dem Dialekt Kukkuzi, der $\tilde{o}$ nicht kennt, sowie Ableitungen. Insgesamt ist die Anzahl der Formen, die den Lautwandel trotz erfüllter Voraussetzungen nicht durchläuft, überschaubar. Von den 13 hier behandelten Wörtern konnten sieben nicht gesichert aufgeklärt werden, wenngleich bei den meisten dieser ungesicherten Belege wohl Fremdeinfluss angesetzt werden kann. Anhand der Lautlehren und anderer wissenschaftlicher Ausführungen lassen sich für den Lautwandel $* e>$ $\tilde{o}$ im Wotischen keine weiteren Kriterien ermitteln, sodass hier die gleichen ansetzbar sind, wie im Estnischen.

\subsubsection{Livisch}

Für das Livische ist festzuhalten, dass in der zweiten Silbe der der Entwicklung * $e>\tilde{o}$ unterliegenden Belege fast ausschließlich $a$ oder ein aus diesem hervorgegangenes sekundäres $\tilde{o}$ vorzufinden sind. Anderen Vokale sind in kaum nennenswerter Anzahl in dieser Position vertreten.

\footnotetext{
${ }^{54}$ Für Beleg (155) wot. ventõizõ̃ 'völlig fremd' wird in VKS nur ein Dialekt als Vertretung genannt, das westwotische Jõgõperä. In anderen Dialekten scheint diese Entsprechung nicht aufzutreten.
} 


$\begin{array}{cc}\text { Vokale der zweiten Silbe } & \text { Anzahl } \\ a & 24 \\ o & 6 \\ u & 3 \\ e & 1 \\ \ddot{a} & 1 \\ \text { Unbestimmbarer } & 1 \\ \text { Hintervokal } & \end{array}$

Einem historischen $* e$ in der ersten Silbe können die nachfolgend aufgeschlüsselten Belege zugeordnet werden:

mit zweiter Silbe, die auf *a zurückgeht:

(2) liv. ỗlma 'Rockschoß',

(106) liv. põ̃zõ 'Busch'

(4) liv. ? ęrā 'Geifer von brünstigen Tieren',

(107) liv. põ̃rand 'Boden',

(5) liv. eráa-māráa 'rote Johannisbeere',

(109) liv. põ̃rna 'Milz'

(14) ? liv. kõlā 'Bogen, Gewölbe, Bucht',

(120) liv. sõbrā 'Freund',

(18) liv. kõlbõ 'taugen',

(122) liv. sõ'ggõl 'Sieb',

(26) liv. kỗrda 'Ordnung, Reihe',

(133) liv. sõzār 'Schwester',

(33) liv. kõ'ddorrz 'Speiche',

(141) liv. tõmbõ 'ziehen',

(53) liv. mȭrda 'Fischreuse',

(148) liv. tỗra 'Teer',

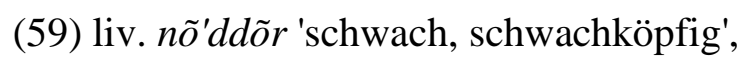

(153) liv. vỗlga 'Schuld',

(60) liv. nõ'ggõl 'Nadel',

(158) liv. vẹrka 'langsam schleppend (z.B.

(65) liv. nō rka 'schwach', Krankheit)',

(74) liv. $\bar{\sigma}^{\prime} d \tilde{o g}$ 'Abend, Westen',

(160) liv. võz $\bar{a}$ 'Trieb, Sprössling, Schößling',

(98) liv. põ'ddorrz 'Elch',

mit zweiter Silbe, die auf $* o$ zurückgeht:

(57) liv. mõtūks 'Auerhuhn, -hahn,

(147) liv. tõ'rmõz 'Eichel',

(67) liv. $\tilde{o}^{\prime} b b i$ 'Pferd',

(154) liv. võ'l 'Zauberer, Hexe',

(115) liv. rõ'ut 'Stangenlänge zwischen zwei (157) liv. võrgõ 'Netz';

\section{Zaunpfosten',}

mit zweiter Silbe, die auf * $u$ zurückgeht:

(70) liv. $\bar{e}^{\prime} g \partial$ 'glühen, schmerzen',

(108) liv. põrm 'Staub, Asche';

(100) liv. $p \overline{\tilde{o}}^{\prime} k t$ 'feiner Staub', 
mit zweiter Silbe, die auf *e zurückgeht:

(77) liv. ó'ldzõ 'hell, grell, klar';

mit zweiter Silbe, die auf $* \ddot{a}$ zurückgeht:

(56) liv. mõtsā 'Wald';

mit nicht näher bestimmbarem Hintervokal:

(55) liv. mõtlõ 'denken, nachdenken'.

Auch für das Livische zeigt sich, dass der Lautwandel $* e>\tilde{o}$ bei hintervokalischer Folgesilbe einzutreten scheint. Im Vergleich zum Estnischen und Wotischen finden sich jedoch vereinzelte Belege, deren zweite Silbe historisch vordervokalisch ist.

\section{ANMERKUNGEN}

(55) An dieser Stelle sei abermals auf den rekonstruierten Stamm urfi. *mettV-verwiesen (LÄGLOS II: 281). Der ursprüngliche Vokal ist unbekannt und wurde wohl synkopiert, das Ableitungssuffix ist hier -lõ (LAANEST 1982: 274). Aufgrund der vorausgegangenen Diskussion ist eine hintervokalische zweite Silbe ansetzbar, vgl. Kapitel 5.3.2.1

(56) Bereits POSTI (1942: 137) schreibt von einer unklaren Entwicklung: Erwartungsgemäß ist in der ersten Silbe *e, vgl. est. mets, gen. - $a$ 'Wald' wot. mettsä id. Zwar nehmen die etymologischen Wörterbücher für diesen Beleg eine (ungesicherte) Entlehnung aus den baltischen Sprachen an (SSA II: 163; EES 281), es liegt jedoch eine glaubwürdige Rekonstruktion eines Wortstammes fiugr. mećä 'Rand, Seite von etwas' vor (UEW I: 269f.), die auf eine ursprünglich vordervokalische zweite Silbe hindeutet. POSTI (1942: 42) setzt an, dass $\ddot{a}$ seinen vordervokalischen Charakter im Livischen in nichtersten Silben bereits früh verloren hat und die Reichweite der Entwicklung ist derart umfangreich, dass ihr ein gewisses Alter zugeschrieben werden kann. Möglich scheint eine so frühe Entwicklung, dass die Bedingungen für die Ausbreitung des neuen Lautes $\tilde{o}$ bereits gegeben waren. Jedoch werfen einige Wörter zu dieser These Fragen auf. Im Livischen gibt es jene, in deren erster Silbe * $e$ unter ähnlichen Bedingungen bewahrt wird. Darunter fallen solche, die zu einem ausreichend alten Wortschatz zählen und in der zweiten Silbe ursprünglich ebenso vordervokalisch waren, vgl. liv. je'llõ 'leben', liv. jemā 'Mutter' und liv. kejā 'Körper, Rumpf' sowie liv. nēḷa 'vier', welche im Rahmen der Gegenprobe näher betrachtet werden (s.u.). Zu notieren ist, dass das vorliegende Sprachmaterial nicht umfassend und eindeutig genug ist, um die Frage nach der Abweichung von (56) zu bewerten. Es scheint, 
als habe man es hierbei mit einer eigenständigen Entwicklung zu tun. Da auch die südestnische Entsprechung abweicht, estS. mõts, gen. mõtsa 'Wald' (s. auch Kapitel 5.3.2.4), diskutiert VIITSO (1978b: 90), dass diese Wortsippe eine Entlehnung mit zwei unterschiedlichen Stammvarianten sei. Im Dialektverbund des Finnischen, Estnischen und Wotischen sei eine vordervokalische Variante entstanden, die im Verbund des Livischen und Südestnischen nicht vorkomme. In letzterem könne die Form *metsa eine spätere Entlehnung sein, deren Konsequenz eine regelmäßige Entwicklung wäre.

$(59,60,98,120,122)$ Die Klusile bleiben, im Gegensatz zu z.B. den finnischen Entsprechungen, erhalten. Vgl. auch die estnischen Entsprechungen in Kapitel 5.3.2.1.

(67) Der Lautwandel der ersten Silbe liegt lediglich im Livischen vor. Es wird ein Stamm urfi. *hepo angesetzt (LÄGLOS I: 95). KETTUNEN (LW XXXIX) geht beim auslautenden $-i$ von der Vertretung eines alten Diphthongs aus (vgl. das von ihm rekonstruierte *hevoinen). Die Annahme eines solchen Diphthongs wird durch die etymologischen Wörterbücher (SSA und EES) allerdings nicht gestützt. Das livische $-i$ kann wohl als spätere Entwicklung angesetzt werden, denn das $* o$ der zweiten Silbe muss bei Eintritt des Lautwandels $* e>\tilde{o}$ noch vorhanden gewesen sein. ${ }^{55} \mathrm{Im}$ Estnischen und Wotischen liegt eine andere Entwicklung vor, da der Laut der ersten Silbe dort in der heutigen Form $o$ ist, vgl. est. hobune 'Pferd' wot. opõnõ id. ${ }^{56}$

(70) Das * $u$ in der zweiten Silbe wurde synkopiert.

(77) Der Beleg geht auf einen Stamm *hele- $\delta A$ zurück. Das historische $* e$ der zweiten Silbe wurde im Verlauf seiner Entwicklung synkopiert (POSTI 1942: 76ff.), womöglich noch vor dem Eintritt von $* e>\tilde{o}$.

\footnotetext{
${ }^{55} \mathrm{Vgl}$. dazu auch LAANEST (1982: 206f.), der anführt, dass $i$ in Suffixen wie fi. -(i)nen- : -(i)se-, zu welchem er

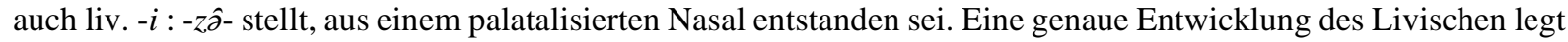
er nicht dar. Der erste Bestandteil des Diphthongs sei im Livischen in nichtersten Silben geschwunden, wenn der zweite $i$ gewesen ist (ebd. 131). Weitere Beispiele, die an jener Stelle genannt werden, sind u.a. liv. pu'nni 'rot' ( fi. punainen id.) und liv. u'ddi 'nebelig' (<*utuinen), sodass bei (154) liv. ó'bbi 'Pferd' wohl von Analogie ausgegangen werden kann.

${ }^{56}$ Die Formen est. hobune 'Pferd' wot. opõnõ id. deuten darauf hin, dass im Estnischen abermals eine Entwicklung $* e>\tilde{o}>o$ vorliegt, vgl. Kapitel 5.3.2.1). Dafür spricht auch est. hebu 'Stute', das mit den anderen Belegen etymologisch zusammengestellt wird (SSA I: 156) und eine Vertretung ist, die nur in einem Teil der Dialekte vorliegt (EMS I: 545). Daher unterliegt sie dem Lautwandel wohl nicht. Die südestnische Form estS. ho|põn', gen.

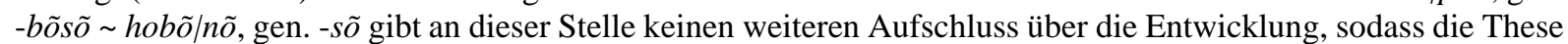
nicht gesichert werden kann. Im Wotischen liegt an dieser Stelle wohl estnischer Einfluss vor.
} 
$(\mathbf{1 0 0}, \mathbf{1 0 8})$ Das *u der zweiten Silbe wurde apokopiert, vgl. zu (100) fi. pehku 'Stroh nach dem Dreschen, Spreu, Streu; (trockener Holz)abfall' est. põ hk, gen. - $u$ 'Kurzstroh, Streu' wot. põhku id. und zu (108) est. põrm, gen. - $u$ 'Staub, Asche' wot. põrmu id.

(106) In der Verbindung * $n s$ wird $*_{n}$ vokalisiert (POSTI 1942: 253). Die Reihenfolge des Ablaufs der Lautwandel in besagter Position ist ferner auch für das Livische aufgrund der geringen Beleglage nicht gesichert festzuhalten, vgl. Kapitel 5.3.2.1. Das õ der zweiten Silbe kann auf *a zurückgeführt werden

(115) ? < est. (LÄGLOS III: 142f.; EEW VIII: 2572; LW 335). Das * $o$ der zweiten Silbe wurde apokopiert, vgl. auch urfi. *rehto.

(133) Vgl. die Erläuterungen zum Estnischen unter Punkt 5.3.2.1

(147) Das *o der zweiten Silbe wurde synkopiert. KETTUNEN (LW 415) setzt für diesen Beleg ein Derivat an.

(154) Das *o der zweiten Silbe wurde apokopiert, vgl. fi. velho 'Hexenmeister, Hexe, Wunderheiler'.

\section{GEGENPROBE}

Die Gegenprobe zum Lautwandel * $e>\tilde{o}$ erfolgte im Livischen unter Berücksichtigung der gleichen Kriterien wie zu den vorausgegangenen beiden Sprachen auf Grundlage der etymologischen Wörterbücher SSA und EES. Folgende Wörter konnten dabei zusammengestellt werden:

1 liv. el'ka 'erschallen; in hohen Tönen klingen; schwappen, klingen, klappern' fi. helkkyä 'in hohen Tönen klingen; schwappen ' SSA (I: 153), EES (73f.)

2 liv. je 'llõ 'leben; tun; arbeiten'

fi. elää 'leben, wohnen' ural. elä- 'leben' (UEW I: 73)

SSA (I: 103f.), EES (60) 
3 liv. jemā 'Mutter; Großmutter; alte Frau; Muttertier; Gebärmutter; Kiel (Boot)' fi. emä 'Muttertier; Tierweibchen; sehr groß, ausgewachsen; Haupt; Gebärmutter' ural. emä 'Mutter, Weib' (UEW I: 74)

SSA (I: 104), EES (60f.)

4 liv.je 'nnõ 'viel'; je 'mbit, jemīn 'mehr'

fi. enä 'groß; viel'

ural. enä 'groß viel' (UEW I: 74)

SSA (I 106), EES (61)

5 liv. kebām, kevām, kievām 'leicht (Gewicht)'

fi. kepeä 'leicht'

fiugr. (? ural.) kepä 'leicht' (UEW I: 146)

SSA (I: 344), EES (139)

6 liv. kejā 'Körper, Rumpf; Gebärmutter; Behältnis'

fi. kehä 'Kreis, Radkranz; [...]'

fiugr. kečä 'Kreis, Ring, Reifen' (UEW I: 141)

SSA (I: 337), EES (141)

7

liv. kerrõ 'sammeln, pflücken' (LW auch ker̀râ 'unter den Einwohnern einsammeln'

(LW 115f.))

fi. kerjätä 'betteln'

fiugr. kerä- 'bitten' (UEW I: 149)

SSA (I 346), EES (145)

8 liv. nēḷa 'vier'

fi. neljä 'vier'

fiugr. ńeljä (neljä) 'vier' (UEW I: 315f.)

SSA (II: 212), EES (310)

9 liv. $r e^{\prime} b b i$ 'Fuchs'

fi. repo 'Fuchs'

osfi. (urfi.) *repäinen (SSA III: 65)

fiugr. repä ( -ćs) 'Fuchs' (UEW I: 423)

SSA (III: 65f.), EES (421f.)

10 liv. sebā-vitsā 'langer Zweig'

fiugr. śepä 'Hals, Nacken' (UEW I: 473) 
fi. sepä 'das nach oben gebogene Vorderende (bzw. ein Teil dess.) des Pferdeschlittens'

SSA (III: 169), in EES nicht behandelt

1, 5-10: Der Abbau der Vokalharmonie ist für die Abweichung ursächlich.

2-4: Eine eigenständige Entwicklung der ersten Silbe liegt vor: $e$ - im Wortanlaut $>$ je- (POSTI 1942: 19), was wohl eine Entwicklung * $e>\tilde{o}$ verhindert hat.

Hinzu kommt ferner ein Beleg, der anhand des Korpus festgestellt werden konnte:

16 liv. ? keldarikki 'Sumpfdotterblume'

fi. kelta 'gelb; Name verschiedener Pflanzen'

16: Die livische Entsprechung gilt als unsicher. Die Wortsippe sei aus dem Baltischen entlehnt, vgl. lit. geltà 'Gelbheit, gelbe Farbe; Gelbsucht, gelbe Garne (meist im Plural)' (SSA I: 342; EES 172; EEW III: 908). Fortwährender Einfluss der baltischen Sprachen scheint hier eine mögliche Begründung für die Abweichung.

Ein wesentlicher Grund für augenscheinliche Abweichungen in der Entwicklung ist abermals der Abbau der Vokalharmonie. Ebenso ist die einzelsprachliche Entwicklung der ersten Silbe wichtig, da sie wohl einen Lautwandel $* e>\tilde{o}$ verhindert hat. Diese These wird in der Gegenprobe anhand der Entwicklung $e_{-}>j e$ - im Wortanlaut gedeckt. Ferner liegen im Livischen Entwicklungen vor, die die erste Silbe derart verändern, dass sie kein Gegenstand der Überprüfung sind, z.B. * $e>$ ie in erster Silbe (PosTi 1942: 19).

Aus dem Gesamtbild der Belege lässt sich schließen, dass die zuvor aufgestellte Vermutung eines regelmäßigen Lautwandels $* e>\tilde{o}$ bei einem Hintervokal in der Folgesilbe auch für das Livische gültig ist, sofern spracheigene Erscheinungen diesen nicht verhindert haben.

\subsubsection{Südestnisch}

Für das Südestnische lässt sich anhand der untersuchten Daten die nachfolgende Verteilung der Laute der zweiten Silbe festhalten. Die Belege, deren zweite Silbe eine Entwicklung $*_{o}>u$ unterliegt (EK ̈̈ 155, 157f.), wurden hier dem historischen Ausgangslaut hinzugerechnet. 


$\begin{array}{cc}\text { Vokale der zweiten Silbe } & \text { Anzahl } \\ a & 28 \\ o & 8 \\ u & 5 \\ \text { Unbestimmbarer Hintervokal } & 1\end{array}$

Die Belege verteilen sich im Südestnischen wie folgt:

mit zweiter Silbe, die auf $* a$ zurückgeht:

(2) estS. hõlm, gen. hõlma 'Rockschoß',

(98) estS. põdorr põtr, gen. põdra 'Elch,

(4) estS. horra(s)silm 'schielendes Auge',

Elentier',

(5) estS. hõrak 'rote Johannisbeere',

(107) estS. põrma(n)d 'Fußboden',

(14) estS. kõla 'Gerät zum Weben eines Gür-

(109) estS. põrn, gen. põrna 'Milz', tels',

(117) estS. rõ̃ngas, gen. .rõnga 'Ring,

(16) estS. kõlla|nõ, gen. -dsõ 'gelb',

Reifen',

(18) estS. . kõlbama 'taugen',

(120) estS. sõbõr sõbr, gen. sõbra 'Freund',

(26) estS. kõrd, gen. kõrra 'Ordnung, Reihe',

(122) estS. sõgõl; sõkl, gen. sõgla 'Sieb',

(33) estS. kõ |tar', gen. -dara 'Speiche',

(133) estS. sõ sar''Schwester',

(48) estS. mõla 'Ruder',

(138) estS. tõbras, gen. .tõpra 'Rind; Vieh',

(53) estS. mõrd, gen. mõrra 'Fischreuse',

(141) estS. .tõmbama 'ziehen',

(59) estS. nõder, gen. nõdra; nõdor 'schwach,

(148) estS. tõrv, gen. tõrva 'Teer',

schwachköpfig',

(152) estS. võhaleht' 'Calla palustris; [...]',

(60) estS. nõgõl; nõkl, gen. nõgla 'Nadel',

(153) estS. võlg, gen. võla 'Schuld',

(65) estS. nõrk, gen. nõrga 'schwach',

(160) estS. võso 'Schössling, Spross';

(74) estS. odak otak 'Abend, Westen',

mit zweiter Silbe, die auf $* o$ zurückgeht:

(3) estS. hõlp, gen. hõlbu 'Leichtigkeit',

(37) estS. lõbu 'Vergnügen',

(52) estS. mõno 'Genuss',

(57) estS. mõtus 'Auerhuhn, -hahn',

(154) estS. võhl, gen. võhlu 'Zauberer, Hexe',

(157) estS. võrk, gen. võrgu 'Netz';

(159) estS. .võrsu|ma, II. Infinitiv-daq 'sprie-

ßen';

(102) estS. põld, gen. põllu 'Feld',

mit zweiter Silbe, die auf * $u$ zurückgeht:

(40) estS. lõhmus 'Linde',

(100) estS. põhk, gen. põhu 'Kurzstroh,

(44) estS. mõdu 'Met',

Streu', 


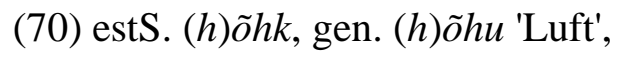
(108) estS. põrm, gen. - $u$ 'Staub, Asche [...]';

mit unbestimmbarem Hintervokal:

(55) estS. .mõtlõma, II. Infinitiv mõtõldaq 'denken, nachdenken';

mit zweiter Silbe, die auf $* \ddot{a}$ zurückgeht:

(56) estS. mõts, gen. mõtsa 'Wald'.

Im Südestnischen zeigt sich ebenfalls, dass die Hintervokale in zweiter Silbe bei einem Lautwandel $* e>\tilde{o}$ in der ersten Silbe dominieren. Lediglich ein Beleg weicht davon ab.

\section{ANMERKUNGEN}

(55) Für das Südestnische sei ebenso auf den rekonstruierten Stamm urfi. *mett $V$ - verwiesen (LÄGLOS II: 281). An diesen Stamm trat ein Ableitungssuffix -le- bzw. -lõ- (vgl. auch LAANEST (1982: 274)). Der ursprüngliche Laut der zweiten Silbe ist nicht rekonstruierbar, eine hintervokalische zweite Silbe kann jedoch angesetzt werden, vgl. auch Kapitel 5.3.2.3.

(56) Bereits bei der Analyse des Livischen wurde die Auffälligkeit dieses Belegs diskutiert vgl. Kapitel 5.3.2.3. Anhand der oben zum Südestnischen angeführten Belege wäre $* e$ in der ersten Silbe hier erwartungsgemäß. Dies ist besonders bemerkenswert, da das Südestnische im Gegensatz zum Livischen die Vokalharmonie kennt. Die Wörter, in denen das Livische ähnliche Bedingungen zu liv. mõtsā vorweist, sind im Südestnischen in der zweiten Silbe allesamt vordervokalisch: liv. je'llõ 'leben' estS. elämä id., liv. jemā 'Mutter' estS. imä id., liv. kejā 'Körper, Rumpf' estS. kihä id. ${ }^{57}$ sowie liv. nēl̦a 'vier' estS. neli, gen. nelä id. Im Südestnischen existieren nur wenige Belege, die den gleichen Bedingungen unterliegen, den Lautwandel gleichwohl nicht vollziehen. Bei den meisten ist von jüngeren Entlehnungen auszugehen, z.B. estS. démokraatia 'Demokratie' oder estS. .petroĺ, gen. - $i$ 'Petroleum', auch Ableitungen können angesetzt werden, vgl. estS. tembota|ma, -q 'Streiche machen, Schabernack treiben' (wohl zu est. temp 'Streich', welches eine unregelmäßige Ableitung von est. tõmmata 'ziehen' sei (EES 523)). Für das Südestnische kommt die von VIITSO (1978b: 90) zum Livischen besprochene Entlehnung der beiden Wortstämme folglich ebenso infrage.

(133) Vgl. die Erläuterungen zur estnischen Entsprechung in Kapitel 5.3.2.1

${ }^{57}$ estS. imä und estS. kihä entwickeln sich andersartig: *e > $i$ (KEEM 2002: 33; vgl. auch EKÄ 128) 
(160) - $o$ ist hier wohl, ähnlich wie im Wotischen, Ableitungssuffix, vgl. Kapitel 5.3.2.2

\section{GEGENPROBE}

Auch im Falle des Südestnischen wurde eine Gegenprobe unternommen, die jedoch aufgrund der geringen etymologischen Behandlung dieser Sprache erschwert wurde. Anhand des Korpus wurden für *e keine Abweichungen festgestellt. Die nachfolgenden Belege konnten dabei zusammengestellt werden:

$1 \quad$ estS. ketrata, keträtä 'spinnen'

fi. kehrä 'Spinnwirtel; Ring'

fi.-wolg. kečrä ( > *kešträ) 'Spindel; ? spinnen' (UEW II: 656)

SSA (I: 336), EES (139f.)

$2 \quad$ estS. kelg, gen. kelgu 'Schlitten'

fi. kelkka 'Schlitten'

*kelkka (LÄGLOS II: 76f.)

SSA (I: 340), EES (143); germ. oder jüngeres Lehnwort (LÄGLOS II: 76f.)

3 estS. keps, gen. kepsu 'Hüpfer'

fi. kepsa 'flink'

SSA (I: 344), EES (144)

$4 \quad$ estS. (Setu) Peko 'Spezielle Gottheit des Getreides'

fi. pekko: pellonpekko 'Gottheit der Gerste (oder eines aus Gerste zubereiteten Getränkes), des Ackers'

SSA (II: 331); in EES nicht behandelt

1: Zwei Formen existieren: estS. keträtä verhält sich erwartungsgemäß, während estS. ketrata, abweicht. Die Abweichung konnte nicht aufgeklärt werden, ist aber wohl sekundär. Es könnte est. Einfluss vorliegen.

2: Die Datierung wird als schwierig erachtet, ein Grund für die Abweichung ist möglicherweise die in LÄGLOS (II: 76f.) angeführte mehrfache Entlehnung.

3: Die Abweichung bleibt ungeklärt, $-u$ ist womöglich eine Ableitung.

4: SSA (II: 331) schlägt verschiedene Entwicklungen vor, u.a. die Parallele zum Eigennamen Pekko. Die Verwendung als Name, auch für eine Gottheit, kann Ursache für das Bewahren des Lautstandes sein. 
Im Südestnischen ist die Vertretung abweichender Belege recht gering. Solche, die eigensprachlichen Entwicklungen unterliegen, wurden hier nicht aufgenommen, da diese den Lautwandel $* e>\tilde{o}$ wohl verhindert haben, vgl. z.B. $* e>i$ (KEEM 2002: 33) oder jene, in denen das Verhältnis est. $e \sim$ estS. $\ddot{a}$ vorliegt, für die jedoch keine Bedingungen angegeben werden (ebd.). Nach der Diskussion ist für das Südestnische die gleiche Entwicklung festzuhalten wie für seine verwandten Sprachen: Ein Lautwandel $* e>\tilde{o}$ tritt bei hintervokalischer Folgesilbe ein, so wie bereits die einschlägigen Belege vermuten ließen. ${ }^{58}$

\subsection{3 $* o>\tilde{o}$}

Der Lautwandel $* o>\tilde{o}$ stellt die zweite große Gruppe der Entwicklungen dar und soll im nachfolgenden Kapitel diskutiert werden.

\subsubsection{Estnisch}

Zunächst gilt es, die Herausbildungen des Lautwandels $* o>\tilde{o}$ im Estnischen zu betrachten. Insgesamt sind 71 der Belege des Korpus für diese Entwicklung einschlägig. Dabei verteilen sich die Laute der zweiten Silbe nicht mehr genauso eindeutig auf Vokale einer bestimmten Qualität, wie das nachfolgende Diagramm zeigt:

$\begin{array}{cc}\text { Vokale der zweiten Silbe } & \text { Anzahl } \\ e & 41 \\ i & 5 \\ a & 16 \\ u & 1 \\ \text { Unbestimmter Vokal } & 8\end{array}$

Diese Verteilung entspricht den nachfolgenden Belegen:

mit zweiter Silbe, die auf $* e$ zurückgeht:

(1) est. hõbe 'Silber',

(8) est. jõgi, gen. jõe 'Fluss',

(15) est. kõle 'trocken',
(83) est. ommelda 'nähen',

(84) est. ong, gen. - $e$ 'Angel(haken)',

(85) est. õnn, gen. - - 'Glück',

(86) est. õo|s, gen. -ne 'Höhle, Höhlung',

\footnotetext{
${ }^{58}$ Da das Südestnische hinsichtlich der Entwicklung von $\tilde{o}$ wissenschaftlich kaum eigenständig behandelt wird, kann hier und im Folgenden kein Vergleich mit Theorien dazu unternommen werden.
} 
(20) est. kõmmeld(i) 'Hohlmesser, Hohlei-

sen',

(24) est. kõr $\mid b$, gen. -velbe 'Urwald',

(25) est. kõrbeda 'anbrennen',

(27) est. kõrend, gen. - $i$ 'Stange',

(28) est. kõrge 'hoch',

(30) est. kõr $\mid s$, gen. -re 'Halm',

(35) est. kõver, gen. - $a$ 'krumm, schief',

(38) est. lõhe 'Lachs',

(39) est. lõhkeda 'bersten, platzen, sich spal-

ten',

(43) est. lõppeda 'beenden',

(49) est. mõlema(d) 'beide',

(51) est. mõni, gen. - $e$ 'jemand',

(61) est. nõges 'Nessel'

(62) est. nõgi, gen. - $e$ 'Ruß',

(64) est. $n \tilde{o} \mid l g$, gen. -le 'Rotz',

(66) est. nõtke 'biegsam, geschmeidig, weich',

(79) est. $\tilde{o} l \mid g$, gen. - $e$ 'Stroh, Halm', mit zweiter Silbe, die auf $* i$ zurückgeht:

(21) est. kõmpida 'einherschreiten, schwerfällig gehen',

(22) est. kõndida 'gehen, spazieren',

(23) est. kõn $\mid t$, gen. - di 'Steifigkeit, Ver-

krüpplung',

mit zweiter Silbe, die auf $* a$ zurückgeht:

(7) est. (h)õrn, gen. - a 'zart, liebevoll',

(19) est. kõmada 'dumpf tönen',

(31) est. kõrv, gen. - $a$ 'Ohr',

(34) est. kõva 'hart, fest, streng',

(54) est. mõrsja 'Braut',

(69) est. ôhata 'seufzen; ausströmen',
(87) est. opppida '(er)lernen',

(90) est. or $\mid s$, gen. -re 'Stange, Hühner-

stange',

(97) est. põdeda 'leiden, krank sein',

(104) est. põlv, gen. -e 'Knie, Generation',

(111) est. põsk, gen. põse 'Wange',

(113) est. põu, gen. - $e$ 'Brust',

(123) est. sõge 'blind',

(124) est. sõl $\mid g$, gen. -le 'Spange',

(125) est. sõlm, gen. - $e$ 'Knoten',

(126) est. sõmer, gen. - $a$ 'Kies, grober Sand',

(130) est. sõre 'undicht, grob',

(132) est. sõrm, gen. - $e$ 'Finger',

(137) est. tõbi, gen. tõve 'Krankheit, Leiden',

(140) est. tõke, gen. tõkke 'Hindernis, Hürde',

(144) est. tõri, gen. tõrve 'Horn, Röhre',

(146) est. $t \tilde{o} r \mid s$, gen. -re 'Kübel, Kufe',

(149) est. tõsi, gen. tõe 'wahr; Wahrheit';
(128) est. sõnn, gen. -i 'Stier',

(129) est. sõnnik, gen. - $u$ 'Mist, Dünger';
(110) est. põrsas 'Ferkel',

(112) est. põtk, gen. - $a$ 'Bein, Schenkel',

(119) est. sõba 'Decke, Bettdecke, wollenes

Umschlagetuch',

(121) est. sõ $\mid d a$, gen. -ja 'Krieg',

(131) est. $s \tilde{r} r \mid g$, gen. - $a$ 'Klaue', 
(78) est. $\tilde{o} l \mid g$, gen. - $a$ 'Schulter',

(80) est. olla, olen-1SGPRÄSAKT 'sein',

(99) est. põh|i, gen. -ja 'Boden, Grund',

(101) est. põlata 'verachten',

mit zweiter Silbe, die auf * $u$ zurückgeht:

(81) est. ôl|u, gen. -le 'Bier';

mit zweiter Silbe, die nicht bestimmbar ist:

(10) est. jõlkuda 'hin und her gehen',

(12) est. $k \tilde{o} h \mid t$, gen. - $u$ 'Bauch',

(46) est. mõjuda 'beeinflussen, wirken',

(47) est. mõksuda 'zusammenschlagen, zusammenstoßen $[\ldots]$,
(135) est. sõtkas 'Bucephala clangula (Schell-

ente)',

(161) est. võtta 'nehmen';

Aufgrund der Belege lässt sich festhalten, dass das Estnische den Lautwandel $* o>\tilde{o}$ in den meisten Fällen zu vollziehen scheint, wenn die Folgesilbe vordervokalisch ist. Allerdings ist das Bild hier nicht mehr genauso eindeutig wie bei der Entwicklung von *e, da der Lautwandel häufig auch dann auftritt, wenn in der zweiten Silbe ein Hintervokal auftritt. Der Vokal $a$ ist hierbei dominant.

\section{ANMERKUNGEN}

\section{Vordervokalische zweite Silbe}

(61) Als Variante auch est. nõgene, steht in etymologischem Zusammenhang mit fi. nokkonen 'Brennnessel'. Ein ursprünglich hintervokalischer Stamm ist wohl ansetzbar (vgl. SSA II: 229), von welchem das Wort mit fi. -nen bzw. est. -ne abgeleitet wurde (vgl. auch LAANEST 1982: 206). Plausibel scheint im Estnischen eine Annäherung des Vokals der zweiten an den der dritten Silbe, sodass in der heutigen Form $e$ vorliegt. Derartige Entwicklungen sind in dieser Sprache aus verschiedenen Bereichen bekannt (EKÄ 181f.).

(81) Als Stamm wird urfi. *olut angesetzt. Die Entwicklung der zweiten Silbe im Genitiv zu $e$ scheint unmotiviert. Für eine Ableitung gibt es keinen Anhaltspunkt. 
(86) Eine Vokalisierung von $*_{n}$ vor $s$ tritt ein, also $* n s>V s$ (EKÄ 104). Die Möglichkeit, dass jene Entwicklung älter sein könnte als die Entstehung des neuen Lautes $\tilde{o}$, wurde bereits bei der Diskussion um den Lautwandel $* e>\tilde{o}$ festgehalten.

(113) KetTUnEN (EKÄ 115) schreibt dem labiodentalen Spiranten $v$ die Eigenschaft zu, gelegentlich als Halbvokal zu funktionieren. Dies äußere sich in der Vertretung $-v-\sim-u-$.

\section{Hintervokalische zweite Silbe}

Die Belege mit $* a$ in zweiter Silbe lassen sich in zwei Gruppen einteilen, einerseits die, in welcher ein labialer Konsonant in direkter Nähe zu $* o$ steht, andererseits jene, in der genau dies nicht der Fall ist. Zu den neun Belegen mit labialer Umgebung der ersten Silbe zählen hier (19), (34), (54), (99), (101), (110), (112), (119) sowie (161). HoLST (2001) zufolge fallen sie unter das Labialitätsgesetz und vollziehen den Lautwandel aufgrund der labialen Laute im direkten Umfeld von * $o$. Seiner Aussage steht die zweite Gruppe der Abweichungen gegenüber (sieben Belege). Diese unterliegt dem Lautwandel ohne labiale Umgebung der ersten Silbe und umfasst die Belege (7), (31), (69), (78), (121), (131) und (135). Dass das Labialitätsgesetz bei fast genauso vielen Belegen nicht eintritt, kann nicht dem Zufall geschuldet sein. Auffällig ist unabhängig von der labialen Umgebung der ersten Silbe, dass der Vokal der Folgesilbe $a$ ist. HoLST (2001: 81) lehnt zwar ab, dass $a$ ausschlaggebend für die Entwicklung der ersten Silbe sei, die Belege lassen allerdings genau diese Theorie zu: Statt labialen Einfluss als Ursache für den Lautwandel von $* o$ zu vermuten, lässt sich schlussfolgern, dass Assimilationsprozesse an der Veränderung der Wörter beteiligt waren und diese die Entwicklung zu õ ausgelöst haben. Das ursprüngliche $* o$ muss sich in diesen Wörtern dem $a$ der zweiten Silbe angenähert haben, ist dabei durch den illabialen Laut entlabialisiert und somit zu $\tilde{o}$ geworden. Auffällig ist hier, dass der Prozess ähnlich ist wie die Entwicklung $*_{o}>\tilde{o}$ bei vordervokalischer Folgesilbe: Entlabialisierung durch Assimilation, aufgelöst durch Laute, die in ihrer Eigenschaft selbst illabial sind. Dass die Assimilation von $*^{*} o$ an das $a$ der Folgesilbe kein abgeschlossener Prozess ist, zeigen weitere Belege, die die Bedingungen für die Entwicklungen zwar erfüllen, * $o$ jedoch bewahren. Allein aus diesem Korpus gehören dazu u.a. (13) est. koht, gen. koha 'Ort, Stelle', (29) est. korjata 'sammeln, pflücken, suchen, [...]' und (32) est. koda, gen. koja 'Hausflur, Haus, Kammer'. ${ }^{59}$ Wäre der Assimilationsprozess abgeschlossen, müsste der Laut $\tilde{o}$ in sämtlichen Wörtern

\footnotetext{
${ }^{59}$ Ferner liegen vor: (68) est. $o k \mid a s$, gen. - $k a$ 'Dorn, Stachel', (71) est. oh|i, gen. ja 'Leine, Zügel', (72) est. oks, gen. - a 'Ast, Zweig', (76) est. oja 'Bach', (82) est. oma 'eigen', (88) est. ora 'Ahle, Pfriem, Metallstift, scharfe Spitze',
} 
mit $* o$ in der ersten und $a$ in der zweiten Silbe vorliegen. Die Belege implizieren stattdessen ein Ende der Ausbreitung.

(80) Das Verb 'sein' stellt einen besonderen Fall dar, wie ein Blick auf das Verbparadigma zeigt $^{60}$ :

$\begin{array}{lll} & \text { Singular } & \text { Plural } \\ \text { 1. Person } & \text { olen } & \text { oleme } \\ \text { 2. Person } & \text { oled } & \text { olete } \\ \text { 3. Person } & \text { on } & \text { on }\end{array}$

Die Infinitivform est. olla vollzieht den Lautwandel nicht, da der Assimilationsprozess bei $a$ in der Folgesilbe hier augenscheinlich nicht eintritt. Bei näherer Analyse des Flexionsparadigmas zeigen sich jedoch auch Formen mit vordervokalischer Folgesilbe, die einen Lautwandel erwarten lassen würden, vgl. z.B. est. olen 'sein-1SGPRÄs' oder est. oli 'sein-3SGPRÄT'. Von Relevanz müssen an dieser Stelle die Formen der 3. Person sein, da sie die höchste Gebrauchshäufigkeit vorweisen. Diese ist wohl der Grund für die Abweichungen der anderen Formen, die analog zu der der 3. Person den Laut der ersten Silbe bewahrt haben.

\section{Unbestimmbare zweite Silbe}

Bei einem Teil der Belege handelt es sich um mit $u$ abgeleitete Verben (vgl. auch KASIK 2015: 165ff.). Die meisten von ihnen sind Onomatopoetika, deren Eigenschaft eine lautliche Variabilität ist. Nach Aufkommen des neuen Lautes $\tilde{o}$ bildeten sich also lautmalerische Parallelformen. Ferner heißt es für das Estnische, dass diese Ableitung $u$ oftmals neue Formen von deskriptiven Stämmen bilde (KASIK 2015: 165). Als onomatopoetisch oder deskriptiv werden gemäß den etymologischen Wörterbüchern bewertet:

(10) vgl. EES (103); in SSA (I: 241) wird eine dialektale Vertretung angesetzt

(47) wird in EES nicht behandelt und in SSA (II: 170) als Wort markiert, das lediglich bei WIEDEMANN vorkommt.

(136) vgl. EES (496),

(89) est. orav, gen. - $a$ 'Eichhörnchen', (91) est. osa 'Teil', (92) est. osta 'kaufen', (93) est. ots, gen. - a 'Ende, Kopf, Spitze', (95) est. oder, gen. odra 'Gerste' und (96) est. osi, gen. osja 'Schachtelhalm'.

${ }^{60} \mathrm{Zu}$ ergänzen ist zudem das Partizip Präsens Aktiv, est. olnud, das beispielsweise in Formen des negierten Präteritums auftritt (est. ma ei olnud 'ich war nicht'). 
Die weiteren abgeleiteten Verben sind:

(46) Die Etymologie des Belegs ist nicht abschließend geklärt, eine gesicherte Rekonstruktion eines Urstammes liegt nicht vor. Von einem ostseefinnischen Stamm in Verbindung zu est. mõista 'verstehen, begreifen' wird ausgegangen (EES 291).

(145) Die Etymologie ist ungeklärt, gesicherte Entsprechungen liegen lediglich im Finnischen und Estnischen vor, ferner eine südestnische Form, die etymologisch nicht behandelt wird. Eine Zusammenstellung mit fi. tora 'Zank, Streit' wird zwar erwogen, ist aber ebenso wenig gesichert (SSA III: 312).

Die beiden verbliebenen Belege, deren Vokal der Folgesilbe in der heutigen Form $u$ ist, sind: (12) ${ }^{62}$ UEW (II: 670) setzt für $u$ eine Ableitung an. Der ursprüngliche Vokal der zweiten Silbe sei unbestimmbar: fi.-perm. koktз 'Bauch' (ebd.). Zwar geht man in der Quelle davon aus, dass es sich um ein Wort aus der finnisch-permischen Sprachschicht handelt, laut SSA (I: 383) sei die Zusammenstellung der ostseefinnischen Belege mit denen der anderen finnougrischen Sprachen unsicher. Fraglich ist, wann das Ableitungssuffix an den Stamm getreten sein soll. Da in allen ostseefinnischen Sprachen ein $u$ in dieser Position vorliegt und das Wort selbst alt ist, kann es sich wohl kaum um eine sehr späte Entwicklung handeln. Die Verhältnisse sind hier nicht eindeutig genug, der Lautwandel scheint unmotiviert.

(75) $\mathrm{Zu}$ nennen ist hier zunächst das Derivationssuffix -ke(ne), welches im Estnischen noch als produktiv gilt (zur Produktivität vgl. KASIK (2015: 251f.)) ${ }^{63}$. In den etymologischen Wörterbüchern wird eine Ableitung von einem Stamm *oha-, von dem auch fi. ohimo 'Schläfe; Leiste' abgeleitet sei (SSA II: 259), diskutiert. Da $u$ in allen etymologisch bestätigten ostseefinnischen

\footnotetext{
${ }^{61}$ Bei Beleg (142) est. tõnguda 'wühlen' kann aufgrund der wotischen Entsprechung wot. tõygata id. zwar erwogen werden, dass die zweite Silbe auf $a$ lautet, diese Hypothese lässt sich abschließend jedoch nicht bestätigen.

${ }^{62}$ Holst (2001: 65) behandelt (12) und dessen finnische Entsprechung fi. kohtu 'Gebärmutter' nicht, da er der Auffassung ist, es hier mit einer unsicheren Entsprechung zu tun zu haben. In UEW (II: 670) begründet man die Zusammengehörigkeit der beiden Entsprechungen dadurch, dass es in den finnougrischen Sprachen oft vorkomme, nah zueinander befindliche Körperteile mit dem gleichen Begriff zu bezeichnen.

${ }^{63}$ KASIK (2015: 252) führt an, dass im Nominativ die Formen - ke und -kene existieren, die Variante -ke allerdings häufiger Verwendung findet. In den flektierten Formen finden sich dagegen meist die, die auf die lange Variante -kene zurückgehen, so z.B. die Genitiv-Form -kese.
} 
Sprachen in der zweiten Silbe auftritt, ist diesem Laut hier ein gewisses Alter zuzuschreiben. ${ }^{64}$ Der Lautwandel der ersten Silbe ist wohl unmotiviert.

\section{GEGENPROBE}

Abermals stellt sich am Ende der Ausführungen die Frage nach einer Gegenprobe, die auch für $* o$ anhand von SSA und EES zusammengestellt wurde. Es gelten ähnliche Kriterien wie schon beim Gegenbeweis von * $e>\sigma^{65}$ Auf dieser Grundlage können die nachfolgenden Belege für das Estnische zusammengestellt werden:

1 est. joriseda 'vor sich hinsingen, gröhlen'

fi. jorista 'undeutlich sprechen, brummen; schwatzen, plaudern'

SSA (I: 242), EES (99)

2 est. klobida 'prügeln, verhauen'

fi. (dial.) klopista 'klingen, klirren'

EES (166); in SSA nicht behandelt

3 est. kobe 'kräftig, stark'

fi. kopea 'stolz, hochmütig; schön, prächtig

SSA (I: 399), EES (167)

4 est. kogemata 'unabsichtlich, aus Versehen; zufällig'

fi. kokea 'erleben, durchmachen, prüfen'

SSA (I: 386), EES (169)

ural. koke- 'sehen, besichtigen, finden' (UEW I: 171); est. koge- hier dial.

5 est. koger, gen. kogre 'Karausche (Carassius carassius)'

fi. kouri 'Karausche (Carassius carassius)'

SSA (I: 414), EES (169)

6 est. kohi, gen. kohi 'impotent; kastriert', SSA auch est. kohitseda 'kastrieren'

fi. kuoha 'Hoden (besonders des Hengstes)'

SSA (I: 439), EES (169)

7 est. kohmida 'trödeln, herumwirtschaften'

fi. (dial.) kohmia, (alt) kohmuta 'tasten, trödeln'

EES (170), in SSA nicht behandelt

\footnotetext{
${ }^{64}$ Zur finnischen Entsprechung vgl. HAKULINEN (1979: 141f.).

${ }^{65} \mathrm{Da}$ die Zweigeteiltheit der Entwicklung von $* o$ bei $a$ in der Folgesilbe bereits festgestellt wurde, wird auf eine Zusammenstellung von Belegen, die dieses Kriterium erfüllen, im Rahmen einer Gegenprobe verzichtet.
} 
est. kolkida 'klopfen, hauen'

fi. kolkata 'klopfen, poltern'

SSA (I: 390), EES (172)

9 est. koll, gen. kolli 'Gespenst, Kinderschreck (Folklore)', laut SSA auch dial. gen. kolju

fi. (dial.; Volkslieder) koljo 'massiges Wesen (Mensch, Tier); Riese, Krankheit bringender Geist, Teufel; klobiger Gegenstand'

SSA (I: 389f.), EES (173)

fiugr. kolja 'böser Geist' (UEW I: 173)

10 est. kolle, gen. kolde 'Teil des Ofens oder Herd, der brennt [...]'

fi. kolle 'unebene Stelle (z.B. einer Wand, Straße); Falte'

SSA (I: 391), EES (173)

11 est. komberdada 'stolpern, humpeln'

fi. kompuroida 'stolpern'

SSA (I: 395), EES (173)

12 est. komme, gen. kombe 'Brauch'

fi. komme 'Streich, Trick; Werkzeug'

SSA (I: 394f.), EES (173)

13 est. komm, gen. kommi 'Schlafbank, Pritsche'

fi. komma 'Rahmen; Häuschen, Hütte; Geigenkörper; Perlmuschel'

SSA (I: 394), in EES nicht behandelt

14 est. kont, gen. kondi 'größter Knochen'

fi. kontti 'Bein, Unterschenkel(knochen)'

SSA (I: 398), EES (175)

15 est. kopsida 'klopfen, pochen', kops, gen. -u 'Schlag, Klopfen'

fi. kopsaa 'klopfen, klappern'

SSA (I: 401), EES (177f.)

16 est. kosida 'freien'

fi. kosia 'freien'

SSA (I: 408); < urgerm. (EES 179), in LÄGLOS nicht behandelt 
est. kosk, gen. kose 'Wasserfall, Stromschnelle'66

fi. koski 'Stromschnelle'

SSA (I: 409), EES (179)

18 est. kott, gen. koti 'Tasche'

fi. kotti 'Beutel; Hodensack; Eihülle (Kuh, Pferd), Gebärmutter, Bauch; Hülle, (Samen)gehäuse; Fischgefäß, kleines Gefäß, Trog (auch als Schleife zum Mistfahren)' urfi. *kotti (LÄGLOS II: 108)

< germ. (SSA I: 413); < urgerm. (EES 180); germ. Lehnwort (LÄGLOS 108f.)

19 est. lodiseda 'gurgeln, glucksen, plätschern'

fi. lotista 'platschen; klappern (Zähne); quatschen'

SSA (II: 95), EES (245)

20 est. lohmida, lohmata 'schlagen, prügeln'

fi. lohmia 'hacken, grobe Stücke abhauen; etw. nachlässig tun; verschwenden, fressen'

SSA (II: 86), EES (246)

21 est. loll, gen. lolli 'dumm verrückt'

fi. dial. lolli 'dumm; dick, schlaff, faul'

SSA (II: 90), EES (247)

22 est. dial. lomm, gen. lommi 'Beule'

fi. lommo 'Delle'

SSA (II: 90), EES (247)

23 est. lontida 'schlendern' wot. lontata 'schlendern, bummeln, sich herumtreiben' EES (248), in SSA nicht behandelt

24 est. lontsida 'schlendern, stapfen'

fi. dial. lonsua 'glucksen; holpern; schwanken, schaukeln'

EES (248), in SSA nicht behandelt

25 est. lopsida 'schlagen'

fi. lopsia 'plappern; essen, schnappen; klopfen, hacken'

SSA (II: 93), EES (251)

${ }^{66}$ SSA (I: 409) zufolge existiert eine Form est. $k \tilde{o} s k$ 'Wasserfall, Stromschnelle', die allein nach WIEDEMANN belegt sei. Daher wurde sie in diesem Kontext nicht beachtet. Gleiches gilt für die Entsprechung wot. Kẹhtšizella (Ortsname im Adessiv), die in VKS und EES nicht belegt ist. 
est. lora 'Gefasel', loriseda 'schwatzen'

fi. lorista 'rieseln'

SSA (II: 93f.), EES (251)

27 est. lorp, gen. lorbi 'Faulpelz, Halunke', lorpida 'herumschlendern, -gehen'

fi. lorppi 'Faulpelz, Dummkopf'

SSA (II: 94), in EES nicht behandelt

28 est. nolk, gen. nolgi 'Jüngelchen, zu großer Mann; junges Tier; nicht ausgewachsener untermaßiger Fisch'

fi. nolkki Bursche, Bengel'

SSA (II: 230), EES (316)

29 est. dial. ogeda 'lehren, raten'

fi. hokea 'reden, schwatzen, immer noch einmal wiederholen; herumerzählen' SSA (I: 170), in EES nicht behandelt

30 est. oherdada 'bohren', dial. uherdada id.

fin. (dial.) uhertaa 'bohren'

SSA (III: 366), EES (334)

31 est. ott, gen. oti 'Bär'

fi. (Volkslieder) ohto 'Bär; großes Tier oder Mensch'

SSA (II: 260), EES (340)

32 est. pobiseda 'murmeln' liv. bobīkšõ 'murmeln, brummen'

? fi. popottaa 'schnell sprechen, plappern; fiepen (Hase)'

EES (377), in SSA (II: 396) wird fi. nicht mit est. und liv. zusammengestellt

33 est. pomiseda 'murmeln, brummen'

fi. pomista 'laut sprechen; dumpf tönen; murmeln'

SSA (II: 393), EES (379)

34 est. rodiseda 'knarren, knirschen'

fi. rotista 'krachen, knattern, prasseln; meckern'

SSA (III: 95), in EES nicht behandelt

35 est. ropsida 'brechen, schwingen'

fi. ropsia '(leicht mit einem Bund grüner Birkenzweige) schlagen; fegen, wedeln;

schlendern; pfuschen'

SSA (III: 93), EES (437) 
est. soliseda 'rieseln, plätschern'

fi. solista 'rieseln, plätschern; hell (er)klingen'

SSA (III: 196), EES (479)

37 est. sohiseda, sohkida 'sausen, rauschen; flüstern, tuscheln'

fi. sohista 'rauschen, zischen; zischeln'

SSA (III: 192), in EES nicht behandelt

38 est. solk, gen. solgi 'Spülwasser'

fi. solkata 'durch Tat oder Wort Unordnung, Verwirrung stiften, besudeln; radebrechen, undeutlich sprechen'

SSA (III: 196), EES (479)

39 est. songida 'wühlen, (durch)wühlen, graben'

fi. sonkia '(durch)wühlen, graben'

SSA (III: 198), EES (480)

$40 \quad$ est. sopp, gen. sopi 'Ende, Winkel'

fi. soppi 'Ecke, Winkel'

SSA (III: 200f.), EES (483)

41 est. sorida 'wühlen, herumkramen'

fi. sorista 'brodeln, rieslen; eifrig, durcheinanderreden; knistern, knattern, rasseln; summen'

SSA (III: 201f.), EES (483)

42 est. sorkida 'stechen, stochern'

fi. sorkkia 'stechen'

EES (483f.), in SSA nicht behandelt

43 est. tokerdada 'verwickelt, verwirrt sein'

fi. tokertaa 'stümpern, etwas mühselig oder ungeschickt tun; mühselig schnitzen'

SSA (III: 306), EES (536)

44 est. toksida 'hämmern'

fi. toksahtaa 'plötzlich auftauchen; (gegen etwas) stoßen; etwas festhalten'

EES (536), in SSA nicht behandelt

45 est. vohmida 'gierig verschlingen'

fi. dial. vohmia 'gierig verschlingen, gierig essen; mit großen Schritten schreiten; herumliegen'

EES (611), in SSA nicht behandelt 
fi. volista 'weinen, heulen, greinen; plätschern; knurren (Magen)'

SSA (III: 469), in EES nicht behandelt

1, 2, 8, 11, 12, 19-29, 32-39, 41-46: Der onomatopoetische bzw. deskriptive Charakter des jeweiligen Wortes kann Grund für die Abweichung sein.

3, 10, 13, 14, 16-18, 40: Warum keine Entwicklung $*^{*}>$ o eintritt ist ungeklärt.

4: Der Beleg sei lexikalisiert (EES 169), in SSA (I: 386) stellt man fi. kopea mit est. dial. kogeda 'fürchten, aufpassen, sich schämen' zusammen, welches $<$ fi.

5: Der Klusil der finnischen Entsprechung ist wohl vokalisiert, warum im Estnischen kein Lautwandel in der ersten Silbe eintritt, kann nicht aufgeklärt werden.

6: Der Beleg trägt in der ersten Silbe ursprünglich einen Langvokal. Während dieser im Finnischen zu einem Diphthong wurde (vgl. LAANEST 1982: 128), wurde er im Estnischen vor intervokalischem $h$ gekürzt (EKÄ 98). Ein Lautwandel *oo > $\tilde{o} \tilde{o}$ liegt nicht vor.

7, 31: Die Verhältnisse sind nicht eindeutig, die historische Vertretung der zweiten Silbe kann nicht ermittelt werden. Für 31 wird von SSA ferner eine Form nach WIEDEMANN angesetzt, nicht jedoch von EES.

9: Fraglich ist, ob nicht die als dialektal angeführte Variante die alte Vertretung ist, vgl. fi. Das Wort wird auf einen fiugr. Stamm zurückgeführt, der in zweiter Silbe hintervokalisch war, sodass ein solcher wohl ursächlich für den Erhalt von *o ist.

15: Ein Hintervokal kann als ursprüngliche Vertretung der zweiten Silbe angesetzt werden, vgl. auch kar. kopsoa 'klopfen, pochen', weps. kopsotada id.

30: Vergleicht man den estnischen Beleg mit weiteren osfi. Sprachen, so ist festzuhalten, dass in der ersten Silbe die Vertretung wohl $* u$ gewesen ist (vgl. EKÄ 129).

Aus dem Korpus lassen sich die nachfolgenden Belege zur Gegenprobe ergänzen:

17 est. kolm, gen. - $e$ 'drei'

fi. kolme 'drei'

73 est. oksendada 'sich übergeben'

fi. oksentaa '(sich) erbrechen'

94 est. otsida 'suchen'

fi. etsiä 'suchen'

urfi. *ecci- / occi- (LÄGLOS I: 58) 
114 est. rohke 'reichlich'

fi. rohkea 'mutig, kühn; (als Mengenangabe) viel, reichlich'

urfi. *rohke- $\delta a$ (LÄGLOS III: 167)

139 est. tohtida 'dürfen'

fi. tohtia 'wagen, sich trauen'

fosfi. *tošt- (SSA III: 303)

(17) Grund für die Abweichung dieses Belegs ist wohl der Status als Zahlwort und dessen Gebrauchshäufigkeit.

(73) Das Verb gilt als deskriptiv bzw. onomatopoetisch.

$(94,114,139)$ Warum $* o$ in der ersten Silbe erhalten bleibt, kann nicht begründet werden.

Die Gegenprobe zum Lautwandel $*_{o}>\tilde{o}$ hat sich als wenig eindeutig herausgestellt und wirft die Frage auf, inwieweit man diese Entwicklung versuchen kann, über eine Gesetzmäßigkeit zu erklären, wenn zwar ein beträchtlicher Anteil der Belege dem Lautwandel unter bestimmten Bedingungen unterliegt, ein aber nicht minder beachtliches Aufkommen an Wörtern existiert, die zwar die Bedingungen des Lautwandels erfüllen, ihn gleichwohl nicht vollziehen.

Auch in der wissenschaftsgeschichtlichen Diskussion ist man in vielen Punkten im Unklaren, was *o anbelangt. WIIK (1986: 42) bietet für die Entwicklung im Estnischen an dieser Stelle keine Lösungsmöglichkeit an und sagt lediglich, dass *o mal bewahrt werde, mal nicht. HoLST (2001: 78) formuliert dagegen eine Regel, dass diese Entwicklung bei vordervokalischer zweiter Silbe eintritt. Für die abweichenden Belege postuliert er ein bereits mehrfach zitiertes Labialitätsgesetz (ebd. 79f.). Die Analyse des Korpus konnte in dieser Sache Klarheit schaffen sowie die Thesen überprüfen: WIIK hat richtig erkannt, dass die Verhältnisse nicht immer eindeutig sind. Die Bedingungen dafür konnten in diesem Rahmen weiter eingegrenzt werden, wenngleich noch immer Fragen unbeantwortet bleiben. HOLSTS Labialitätsgesetz hat sich dabei als nicht gültig herausgestellt, da die Assimilationstendenz von *o nicht nur auf die Beeinflussung durch die vordervokalische zweite Silbe beschränkt und nicht aus der labialen Umgebung der Silbe zu erklären ist.

\subsubsection{Wotisch}

Insgesamt liegen im Wotischen 67 einschlägige Belege für den Lautwandel * $o>\tilde{o}$ vor. Davon sind 34 in der Folgesilbe vordervokalisch, während 29 hintervokalisch sind. Sie stehen also 
zahlenmäßig in einem recht ausgewogenen Verhältnis zueinander. In einem geringfügigen Teil der Belege konnte der Stammvokal nicht ermittelt werden. Die Vokale der zweiten Silbe verteilen sich bei diesem Lautwandel im Wotischen gemäß der folgenden Tabelle.

$\begin{array}{cc}\text { Vokale der zweiten Silbe } & \text { Anzahl } \\ e & 33 \\ i & 1 \\ a & 27 \\ o & 1 \\ u & 1 \\ \text { Unbestimmbarer Vokal } & 4\end{array}$

Die Belege sind wie folgt:

mit zweiter Silbe, die auf *e zurückgeht:

(1) wot. õpõa 'Silber',

(84) wot. õntši, gen. õndžõ õ 'Angel(haken)',

(8) wot. jõtši, gen. jõgõ õ 'Fluss',

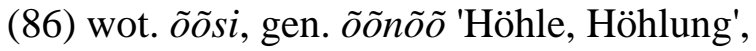

(14) wot. kõla 'trocken, kalt',

(87) wot. öppõa '(er)lernen',

(14) wot. kõlmõ D 'drei',

(90) wot. orrsi, gen. orrrõo 'Stange, Hühner-

(27) wot. kõrõta 'Tragholz. Schulterjoch', stange',

(28) wot. kõrkõa 'hoch',

(101) wot. põlgõt/taa 'mit Pferden tretend das

(30) wot. kõrsi, gen. kõrrõ̃ 'Halm',

Korn dreschen',

(38) wot. lõhi, gen. lõhõ ' 'Lachs',

(104) wot. põlvi, gen. põlvõ̃ 'Knie, Genera-

(39) wot. lõhgõta 'bersten, platzen, sich tion',

spalten',

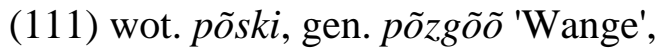

(43) wot. lõpõttaa 'beenden',

(112) wot. põtkõa 'treten',

(49) wot. mõlõpaD 'beide',

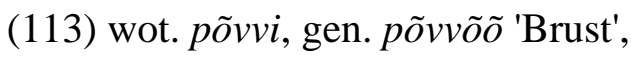

(51) wot. mõni, gen. mõnõ $\tilde{o}$ 'mancher',

(123) wot. sõkõa 'blind',

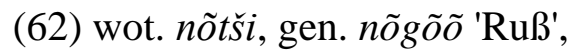

(125) wot. sõlm $\mid u$, gen. - $u$ 'Knoten',

(66) wot. nõtkõa 'biegsam, geschmeidig,

(126) wot. sõmõrõ 'Kies, grober Sand', weich',

(73) wot. ôhsõta 'sich übergeben', (132) wot. sõrmi, gen. sõrmõ õ 'Finger',

(79) wot. õltši, gen. õlgõ̃ 'Stroh, Halm', (140) wot. tõgõ, gen. tõkõo 'Fischwehr,

(83) wot. õmmõlla 'nähen', Fischzaun',

(85) wot. õnni, gen. õnñ̃ ô 'Glück',

(149) wot. tõsi, gen. tõ̃ 'wahr; Wahrheit'; 
mit zweiter Silbe, die auf $* i$ zurückgeht:

(94) wot. ottsia 'suchen',

mit zweiter Silbe, die auf $* a$ zurückgeht:

(9) wot. jõka 'jeder',

(13) wot. kõhta ' Ort, Stelle',

(86) wot. $\tilde{o} \tilde{s} i$, gen. $\tilde{o} \tilde{o n o \tilde{o} ~ ' H o ̈ h l e, ~ H o ̈ h l u n g ', ~}$

(15) wot. kõla 'trocken, kalt',

(88) wot. orra 'Ahle, Pfriem, Metallstift,

(22) wot. kõntaassa 'hin und zurück gehen, scharfe Spitze',

spazieren',

(89) wot. orrava 'Eichhörnchen',

(29) wot. kõrjata 'verstecken, (sich) bede-

(91) wot. õsa 'Teil',

cken; sammeln, pflücken',

(92) wot. ossaa 'kaufen',

(31) wot. kõrva 'Ohr',

(93) wot. õttsa 'Kopf, Ende',

(32) wot. kõta 'Hausflur, Haus, Kammer',

(95) wot. õzra 'Gerste',

(34) wot. kõva 'hart, fest, streng',

(96) wot. õzża 'Schachtelhalm',

(68) wot. ogaz 'Dorn, Stachel',

(99) wot. põhja 'Boden, Grund',

(71) wot. ôhja 'Leine, Zügel',

(72) wot. ôhsa 'Ast, Zweig',

(76) wot. oja 'Bach',

(80) wot. ôlla 'sein',

(82) wot. óma 'eigen',

(110) wot. põrzaz 'Ferkel',

(114) wot. rõhgaa 'sehr, sehr viel, ziemlich',

(119) wot. sõpa 'Bekleidung, Kleider',

(121) wot. sõta, gen. sõaa 'Krieg',

(142) wot. tõygata 'wühlen',

(161) wot. võttaa 'nehmen';

mit zweiter Silbe, die auf $* o$ zurückgeht:

(61) wot. nõkõn 'Nessel';

mit zweiter Silbe, die auf $* u$ zurïckgeht:

(81) õlu|D, gen. $-u$ 'Bier'

mit zweiter Silbe, die nicht bestimmbar ist:

(12) wot. kõhu|z, gen. -hsõ̃ 'Taille',

(136) wot. sõtkoa/-ua 'treten, kneten',

(75) wot. ôhuD 'dünn',

(139) wot. tõhtia 'dürfen, können'.

Auffällig ist auch hier die Verteilung der zweiten Silbe. Meist ist $* e$ der (ursprüngliche) Laut in dieser Position, während $i$ nur in einem Bruchteil der Belege auftritt. ${ }^{67}$ Auch im Wotischen ist es $* a$, das die zweitstärkste Vertretung ausmacht.

\footnotetext{
${ }^{67} \mathrm{Zu}$ beachten ist hierbei der alte Stammvokal $* e$. Bei $i$ in den Nominativformen handelt es sich meist um eine Entwicklung $* e>i$ im Auslaut (vgl. dazu auch LAANEST (1982: 116)). Ferner gibt es eine sekundäre Entwicklung $*_{e}>\tilde{o}$ in nichtersten Silben, die es zu berücksichtigen gilt. Belege, die diesen Lautwandeln unterliegen, wurden dem eigentlichen Stammvokal *e hinzugezählt.
} 


\section{ANMERKUNGEN}

\section{Vordervokalische zweite Silbe}

(15) Anzusetzen ist ein Stamm *kole- $\delta a$. Warum das *e der zweiten Silbe im Wotischen geschwunden ist, kann nicht begründet werden. Andere Belege mit ähnlicher Struktur bewahren den Vokal in dieser Position normalerweise, vgl. z.B. (1) wot. õpõa 'Silber', (28) wot. kõrkõa 'hoch'. Eine Synkope komme KETTUNEN (VKÄ 145) zufolge im Wotischen kaum vor, der Vokal in unbetonten oder schnell ausgesprochenen Silben könne in seltenen Fällen dennoch schwinden. Dieser Beleg scheint solch ein ungewöhnlicher Fall zu sein.

(86) $* n$ wird in der Verbindung *ns vokalisiert (VKÄ 92f.). Über das potenzielle Alter des Lautwandels wurde bereits in den vorausgehenden Kapiteln referiert. Zur estnischen Entsprechung vgl. Kapitel 5.3.3.1.

(94) Rekonstruiert werden für diese Wortsippe urfi. *ecci- und *occi- (LÄGLOS I: 58). Die Ausprägungen der südostseefinnischen Sprachen gehen wohl auf die Form mit $* o$ in der ersten Silbe zurück. Dieses sei eine sekundäre Entwicklung (ebd.).

(125) $-u$ ist ein Ableitungssuffix (ARISTE 1968: 116). Der Beleg kann auf einen vordervokalischen Urstamm fiugr. ćolme 'Knoten, Bündel; binden' zurückgeführt werden (UEW I: 38). Auch die finnischen und estnischen Entsprechungen deuten auf eine sekundäre Ableitung hin, vgl. est. sõlm, gen. - $e$ 'Knoten' fi. solmu, solmi id.

\section{Hintervokalische zweite Silbe}

Mit insgesamt 32 Belegen $^{68}$ ist die Vertretung jener mit Hintervokal $a$ in der Folgesilbe im Wotischen zahlenmäßig beinahe so groß wie die der vordervokalischen Belege. In direktem Kontakt zu einem labialen Laut in der ersten Silbe stehen im Wotischen (31), (34), (82), (99), (110), (119), (161). Mit lediglich sieben von 32 Belegen kann davon ausgegangen werden, dass das Labialitätsgesetz nach HOLST (2001) für die Entwicklung nicht gilt. Die labiale Umgebung

\footnotetext{
${ }^{68}$ Die Entwicklung von (79) wot. tõygata 'wühlen' ist nicht gänzlich gesichert (vgl. die Diskussion um die estnische Entsprechung in Kapitel 6.3.4.1), * $a$ kann in der zweiten Silbe wohl angesetzt werden.
} 
von $* o$ kann also auch im Wotischen nicht der Grund für den Lautwandel sein. Stützen lässt sich eine solche Annahme auch durch die Ergebnisse der Untersuchung im Estnischen. ${ }^{69}$ Deutlich wird, dass es sich im Wotischen ebenfalls um einen Fall von einsetzender Assimilation handelt: Das $* o$ der ersten Silbe hat sich durch den Lautwandel $*_{o}>\tilde{o} \operatorname{dem} a \operatorname{der}$ Folgesilbe angenähert und wurde illabialisiert, vgl. Kapitel 5.3.3.1. Die wotischen Belege stützen die Annahme, dass das Labialitätsgesetz nach HoLST keine ausreichende Erklärung für den Lautwandel bietet. Die Menge an wotischen Entsprechungen, deren zweite Silbe den Vokal $a$ trägt, den Lautwandel aber nicht durchlaufen haben, impliziert gleichzeitig auch in dieser Sprache, dass die Assimilation ein nicht abgeschlossener Prozess ist. ${ }^{70}$ Belege gleicher Bedingungen ohne Lautwandel sind z.B. (131) wot. sorkka 'Klaue' sowie außerhalb des Korpus u.a. wot. loma 'Barre, Stange' oder wot. porata 'zerschlagen; steif werden, erstarren, lahm werden'. Die Wörter wot. loma und wot. porata sprechen ferner gegen die Richtigkeit des Labialitätsgesetzes. Hätte es im Wotischen Gültigkeit gehabt, hätte der Lautwandel in diesen Belegen vollzogen werden müssen.

(80) Das Verb 'sein' ist abermals ein interessanter Fall. Während sich im Estnischen die Formen mit $*_{o}$ in der ersten Silbe durchgesetzt haben (vgl. Kapitel 5.3.3.1), findet man im Wotischen ein lautlich gemischtes Paradigma, in welchem die Formen mit o zahlenmäßig stark überwiegen. Lediglich in der 3. Person Präsens Singular und Plural existieren solche, in denen $* o$ bewahrt wurde. Es sind gleichzeitig jene mit hoher Gebrauchshäufigkeit, sodass der Erhalt der ursprünglichen Lautung nicht verwundert. Das Paradigma für Präsens und Imperfekt gestaltet sich nach ARISTE (1968: 68) folgendermaßen:

Präsens

$\mathrm{Sg}$.

1. Person miä eูren $\sim \bar{e} n$

2. Person siä еле $D \sim \bar{e} D$

3. Person tämä on
Imperfekt

Pl. Sg. Pl.

$$
\begin{aligned}
& \text { тӧ еллетта е èmma ellin elimma } \\
& \text { tö éлetta } \sim \text { ętta ellid elitta } \\
& \text { nämä omaD onvaD ovaD eli elivaD }
\end{aligned}
$$

(61) In diesem ursprünglich dreisilbigen Wort schwindet zuerst das auslautende $-n$, ehe das in den Auslaut geratene - $e$ - des Suffixes -nen entfällt (VKÄ 145). Die Entwicklung von $*_{o}>\tilde{o}$ in

\footnotetext{
${ }^{69}$ Nicht unter das Gesetz fallen folglich ebenso mit * $a$ in der zweiten Silbe und ohne labiale Umgebung die Belege (9), (13), (22), (29), (32), (68), (71), (72), (76), (80), (88), (89), (91), (92), (93), (95), (96), (114), (121), (142).

${ }^{70}$ Dieses Ergebnis entkräftet zugleich HoLSTS (2001: 88) Annahme, dass der Lautwandel $*_{o}>\tilde{o}$ bei einem $a$ in der zweiten Silbe im Wotischen regelmäßig sei.
} 
der zweiten Silbe ist sekundär, da $*^{o}$ in dieser Position normalerweise bewahrt wird (vgl. z.B. (102) wot. põlto 'Feld, Ackerstreifen') (ebd. 141f.). Der Auslöser des Lautwandels ist lautgesetzlich nicht zu erklären. Von estnischem Einfluss muss in diesem Fall ausgegangen werden, vgl. die Entsprechung est. nõges 'Nessel' (Kapitel 5.3.3.1).

(81) Der Beleg geht auf einen Stamm urfi. *olut zurück. Der Lautwandel scheint hier unmotiviert aufzutreten, eine Beeinflussung durch das Estnische ist allerdings erwartbar.

\section{Unbestimmbare zweite Silbe}

(12) - uz ist ein Ableitungssuffix (ARISTE 1968: 115). Der ursprüngliche Stammvokal ist nicht zu ermitteln, die anderen ostseefinnischen Sprachen deuten auf ein $u$ in dieser Position hin (vgl. auch UEW (II: 670) und SSA (I: 383)). Estnischer Einfluss ist aufgrund des Lautwandels der ersten Silbe wohl ansetzbar.

(75) Im Vergleich zu den anderen osfi. Sprachen muss bei $u$ von einem höheren Alter ausgegangen werden. Der Lautwandel scheint unmotiviert, ist womöglich auf estnischen Einfluss zurückzuführen, vgl auch Kapitel 5.3.3.1 zum Estnischen.

(136) Zwei Varianten liegen vor. $u$ ist eine Ableitung (ARISTE 1968: 119), gleiches gilt wohl auch für die Variante mit $o$. In den etymologischen Wörterbüchern (EES 496) wird eine onomatopoetisch beeinflusste Form angesetzt, die hier der Grund für die Abweichung der ersten Silbe sein kann.

(139) Der Beleg wird durch das Suffix $i$ abgeleitet, dessen Grundverb unbekannt sei (LAANEST 1982: 275). Als Urstamm wird urfi. *tošt- erwogen, der ursprüngliche Vokal der zweiten Silbe ist unbekannt (SSA III: 303). Ferner wird davon ausgegangen, dass dieses Wort aus dem Estnischen ins Wotische gelangt ist (ebd.). Soweit dies stimmt, ist fraglich, warum der Lautwandel entgegen der estnischen Entsprechung vollzogen wurde, vgl. est. tohtida 'dürfen'.

\section{GEGENPROBE}

Für die Gegenprobe zum Wotischen konnten die nachfolgenden Belege auf Grundlage von SSA und EES zusammengestellt werden. Aus dem Korpus sind keine Belege zu *o zu ergänzen. 
1 wot. (Kukk) hokkia 'reden, schwatzen, immer noch einmal wiederholen; herumerzählen'

fi. hokea 'reden, schwatzen, immer noch einmal wiederholen; herumerzählen' SSA (I: 170), in EES nicht behandelt

2 wot. klopisa 'klappern; klopfen'

fi. (dial.) klopista 'klingen, klirren' EES (166); in SSA nicht behandelt

3 wot. kolisa 'klopfen, umziehen'

fi. kolata 'rumpeln, poltern'

SSA (I 388), EES (171f.)

4 wot. kosia, (Kukk) koźźoa 'freien'

fi. kosia 'freien'

SSA (I: 408); < urgerm. (EES 179); in LÄGLOS nicht behandelt

5 wot. kotti 'Tasche'

fi. kotti 'Beutel; Hodensack; Eihülle (Kuh, Pferd), Gebärmutter, Bauch; Hülle, (Samen)gehäuse; Fischgefäß, kleines Gefäß, Trog (auch als Schleife zum Mistfahren)' urfi. *kotti (LÄGLOS II: 108)

< germ. (SSA I: 413); < urgerm. (EES 180); germ. Lehnwort (LÄGLOS 108f.)

fi.-perm. konte 'Korb, Ranzen aus Birkenrinde' (UEW I: 177)

$6 \quad$ wot. (Kukk) lopisa 'Unpassendes reden'

fi. lopista 'schwatzen, quatschen'

SSA (II: 92), EES (244)

7 wot. lotise 'platschen, klappern (Zähne); quatschen'

fi. lotista 'platschen; klappern (Zähne); quatschen' SSA (II: 95), EES (245)

8 wot. lopsia 'schlagen, prügeln, Ohrfeige geben' klopsahtaa 'krachen'

fi. lopsia 'plappern; essen, schnappen; klopfen, hacken'

SSA (II: 93), EES (251)

9 wot. lorissa 'schwatzen'

fi. lorista 'rieseln'

SSA (II: 93f.), EES (251)

10 wot. pomisa 'murmeln, brummen, dröhnen'

fi. pomista 'laut sprechen; dumpf tönen; murmeln' 
SSA (II: 393), EES (379)

11 wot. (Tsv.) ropsia 'schwingen, schütteln, rütteln'

fi. ropsia '(leicht mit einem Bund grüner Birkenzweige) schlagen; fegen, wedeln;

schlendern; pfuschen;'.

SSA (III: 93), EES (437)

12 wot. šolata, šolisa 'rieseln, plätschern; hell (er)klingen'

fi. solista 'rieseln, plätschern; hell (er)klingen'

SSA (III: 196), EES (479)

13 wot. šohisa 'sausen, brausen'

fi. sohista 'rauschen, zischen; zischeln'

SSA (III: 192), in EES nicht behandelt

14 wot. solkki 'Spülwasser'

fi. solkata 'durch Tat oder Wort Unordnung, Verwirrung stiften, besudeln; radebrechen, undeutlich sprechen'

SSA (III: 196), EES (479)

15 wot. soria 'wühlen, herumkramen', SSA: šorisa 'sausen, brausen, rauschen; strömen, rieseln, plätschern'

fi. sorista 'brodeln, rieslen; eifrig, durcheinanderreden; knistern, knattern, rasseln; summen'

SSA (III: 201f.), EES (483)

16 wot. (Tsv) volize- 'murmeln, sprudeln, rauschen, sprudeln, glucksen'

fi. volista 'weinen, heulen, greinen; plätschern; knurren (Magen)'

SSA (III: 469), in EES nicht behandelt

1, 6: Für diese Belege wird der Dialekt Kukkuzi angesetzt, in dem $\tilde{o}$ in der ersten Silbe nicht auftritt.

2, 3, 7-10, 12-15: Als Grund für die Abweichung ist der onomatopoetische bzw. deskriptive Wortcharakter ansetzbar.

4: Eine der beiden angeführten Varianten stammt aus dem Dialekt Kukkuzi, vgl 1. Die Abweichung der anderen Variante könnte auf Fremdeinfluss zurückzuführen sein.

5: Der Grund dafür, dass der Lautwandel nicht eintritt, konnte nicht ermittelt werden, Fremdeinfluss scheint möglich. 
11, 16: Einerseits haben die Belege onomatopoetischen Charakter, andererseits sind sie unsicher, da sie nach TSVETKOV notiert sind.

Ferner lassen sich aus dem Korpus die nachfolgenden Belege hier zuordnen:

35 wot. kovera 'krumm, schief'

fi. kovera 'konkav, ausgehöhlt'

130 wot. sorõa 'groß, grob, undicht'

fi. sorea 'ebenmäßig, schön'

(35) Bereits an der lautlichen Gestalt des Belegs fällt auf, dass das $e$ der zweiten Silbe erhalten ist; der sekundäre Lautwandel zu õ wird nicht durchgeführt. Dies könnte ein Anzeichen für Fremdeinfluss sein. In SSA (I: 415) setzt man den Dialekt Kukkuzi an. Dieser gilt gemeinhin als stark durch das Ingrische beeinflusst, sodass viele Eigenschaften des Wotischen nicht mehr klar zu erkennen seien (ARISTE 1968: V). Die Formen weisen hier deutliche Ähnlichkeiten zum Ingrischen auf, vgl. ing. kovvēra 'krumm, gebogen, gekrümmt', ing. kovertā 'biegen, krümmen, drücken; eine Kurve machen, ausweichen' (SSA I: 415). ${ }^{71}$ VKS (480) nennt als Ort der Aufzeichnung der wotischen Belege stattdessen u.a. die Dialekte Mati, Liivtšülä, Luuditsa und Jõgõperä, was Fremdeinfluss freilich nicht ausschließt.

(130) Der Lautwandel tritt nicht ein, Fremdeinfluss kann auch hier erwogen werden.

Die Gegenprobe für das Wotische fällt überschaubar aus. Betrachtet man die Belege genauer, so stammen sie oftmals aus dem Dialekt Kukkuzi oder gelten als Onomatopoetika, auch Fremdeinfluss kommt infrage. Wenngleich einige Abweichungen vorliegen, kann angenommen werden, dass der Lautwandel $*_{o}>\tilde{o}$ bei vordervokalischer Folgesilbe oder $a$ in dieser Position meist eintritt. Anhand anderer wissenschaftlicher Arbeiten lassen sich keine weiteren Kriterien ergänzen.

\subsubsection{Livisch}

Besonders interessant ist die Betrachtung des Lautwandels $* o>\tilde{o}$ im Livischen. Insgesamt sind lediglich neun Belege in dieser Sprache einschlägig, die sich auf nur zwei unterschiedliche Vokale als Vertretung der zweiten Silbe verteilen:

\footnotetext{
${ }^{71} \mathrm{Vgl}$. dazu auch ing. kov̌vēraa 'krumm, gebogen, gekrümmt', welches von NIRVI (IMS: 201) in verschiedenen Varianten für unterschiedliche ingrische Dialekte festgehalten wird, hier z.B. Repola.
} 


$\begin{array}{cc}\text { Vokale der zweiten Silbe } & \text { Anzahl } \\ e & 5 \\ a & 4\end{array}$

Als Belege, die dem Lautwandel unterliegen, sind zunächst wie folgt zu nennen: mit zweiter Silbe, die auf $* e$ zurückzuführen ist:

(1) liv. ó 'bdõ 'Silber', (66) liv. nõtkõ 'biegen, beugen, krümmen', (15) liv. $k \tilde{o}^{\prime} l$ 'trocken, kalt', (86) liv. $\tilde{\tilde{o} n}$ 'Höhle, Höhlung';

(35) liv. kõ'urõ 'krumm, schief', mit zweiter Silbe, die auf $* a$ zurückzuführen ist:

(34) liv. kõva 'hart, fest, streng', (146) liv. tõ̃rdaz 'Kübel, Kufe', (131) liv. sȭrga 'Furche der Klaue', (161) liv. võttõ 'nehmen'.

Es lässt sich anhand der vorliegenden Belege kaum eine konkrete Aussage über den Lautwandel in dieser Sprache treffen, daher muss von anderen Entwicklungen ausgegangen werden, deren Hintergrund in einem späteren Teil dieses Kapitels erörtert werden soll.

\section{ANMERKUNGEN}

(1) Ein Stamm fosfi. *šope- $\delta a$ 'weich' (SSA I: 172) ist anzusetzen. Das historische *e der zweiten Silbe wurde im Zuge der sprachinternen Entwicklungen synkopiert.

(15) Als Stamm ist für diesen Beleg *kole- anzusetzen. KeTTUNEN (LW 118) vermutet bei der livischen Entsprechung eine (unsichere) Entlehnung aus dem Estnischen. Diese lässt sich anhand der etymologischen Wörterbücher nicht bestätigen (vgl. SSA I: 389).

(34) Der Beleg wird von Posti (1942: 11) durch den Lautwandel $*_{o}>\tilde{o}$ vor $v$ erklärt.

(35) Auch hier wurde $* e$ in der zweiten Silbe synkopiert. Laut Posti (1942: 11) ist abermals *o $>\tilde{o}$ vor $v$ ansetzbar. Es folgte $* v>u$, da $v$ im Zuge der Entwicklung in den Silbenauslaut geraten ist (ebd. 265).

(86) Die etymologischen Wörterbücher setzen eine (mögliche) Entlehnung aus dem Estnischen an (vgl. SSA (II: 268) und EES (628)). Der Vordervokal * $e$ kann für die zweite Silbe angesetzt 
werden, vgl. fi. onsi, gen. onnen 'hohle Stelle, Höhlung; hohl, leer' est. õó|s, gen. -ne 'Höhle, Höhlung'. Der vermeintliche Langvokal der ersten Silbe ist im Estnischen auf eine Vokalisierung des $* n$ zurückführbar (EKÄ 104). Die Vokalisierung von $* n$ in der Verbindung * $n s$ beschreibt PosTi (1942: 253) ferner auch für das Livische.

$(\mathbf{1 3 1}, \mathbf{1 4 6}, \mathbf{1 6 1})$ Unklar ist, warum der Lautwandel in diesen Belegen vollzogen wird. Ob auch im Livischen die Assimilation von $* o$ bei $a$ in der zweiten Silbe einsetzt, ist aufgrund der geringen Menge an Belegen kaum mit Sicherheit zu sagen. Stattdessen ist hier von einer unmotivierten Entwicklung auszugehen.

Wie eingangs notiert, ist die Anzahl an einschlägigen Belegen im Livischen bei diesem Lautwandel überschaubar. Bereits ITKONEN (1945: 181f.) erkennt, dass hier andere Verhältnisse vorliegen als im Estnischen und Wotischen, expliziert diesen Umstand aber nicht genauer. Die Abweichung ist jedoch wesentlich: Das Livische unterliegt phonetischen Entwicklungen, die den Lautwandel $* o>\tilde{o}$ verhindert haben. Für die Untersuchung von $\tilde{o}$ sind dabei drei Entwicklungen wesentlich.

Eine große Gruppe mit insgesamt 16 Belegen allein im Korpus dieser Betrachtung ist jene, die in der ersten Silbe einen Lautwandel $*_{o}>$ uo vollzieht. POSTI (1942: 6) bezeichnet diese Erscheinung als eine, die ,im [A]llgemeinen“ für das Livische gelte - ein Umstand, der sich auch in der hohen Anzahl an Belegen in dieser Untersuchung widerspiegelt. Zu den genauen Bedingungen, wann dieser Lautwandel eintritt, äußert sich POSTI ${ }^{72}$ nicht, wohl auch, weil es ein derart umfangreiches Phänomen ist, das die Regel und nicht eine Ausnahme zu sein scheint. Aufgrund der hohen Frequenz des Lautwandels ist davon auszugehen, dass er älter ist als das Einsetzen von $*_{o}>\tilde{o}$, sodass die weitere Ausbreitung von $\tilde{o}$ im Livischen verhindert wurde. Für diese These spricht neben einem geringen Auftreten von $\tilde{o}$ in dieser Sprache auch der Umstand, dass die meisten untersuchten Wörter zu $*_{o}$ der Entwicklung $*_{o}>$ u unterliegen. Belege dieses Korpus, die dieser Gruppe zugeordnet werden können, sind:

(13) liv. ku'odi 'gerade, ehrlich',

(110) liv. pūoraz 'Ferkel',

(17) liv. kuolm 'drei',

(121) liv. suodā 'Krieg',

(25) liv. kuorbõ 'anbrennen',

(124) liv. suolg 'Spange',

(28) liv. kuordõ 'hoch',

(125) liv. suolm 'Knoten',

\footnotetext{
${ }^{72} \mathrm{Zu}$ den Fällen, in denen eine andere Entwicklung als $* o>$ uo vorliegt, vgl. POSTI (1942: 7-11).
} 
(29) liv. kuor'ŕs 'sammeln, pflücken',

(71) liv. $\bar{u}^{\prime} o l m o \tilde{z}$ 'Leine, Zügel',

(99) liv. $p \bar{u}$ 'oj 'Boden, Grund',

(104) liv. pūola 'Knie, Generation',
(132) liv. suorm 'Finger',

(136) liv. suotkõ 'treten, kneten',

(139) liv. tū'od̦ $\tilde{o}$ 'dürfen',

(149) liv. $t^{u} o^{\prime} i z ̌$ 'wahr; Wahrheit'.

Hinzu kommt eine Gruppe mit 22 Belegen dieses Korpus. Hier tritt der Vokal $\dot{o}$ auf. PostI (1942: 129) ist der Auffassung, dass die labialen Konsonanten im Wortanlaut einen Lautwandel $o,{ }^{u} O>g$ ausgelöst haben. ${ }^{73}$ Die Belege dieser Gruppe sind:

(7) liv. vörna 'sanft, zart, liebevoll',

(92) liv. vóstõ 'kaufen',

(46) liv. mojjõ 'fühlen, wahrnehmen, wirken',

(93) liv. vōntsa/vūontsa 'Stirn, hervorstehen-

(49) liv. mó'lmõd 'beide', der oberer Teil der Stirnseite von etw.',

(76) liv. $v(u)_{\text {oj }} \bar{a}$, vojja 'mit Wasser gefüllte

Vertiefung, Pfütze',

(94) liv. vòtšõ 'suchen',

(79) liv. vòlg 'Stroh, Halm',

(95) liv. vó'ddõrz/v(u)o'draz 'Gerste',

(80) liv. vōlda 'sein',

(96) liv. vỏža 'Schachtelhalm',

(81) liv. vó'l 'Bier',

(85) vón 'Glück',

(97) liv. $p \dot{o} ' d d \tilde{o}$ 'schmerzen',

(88) liv. vörā 'Spitze', (101) liv. pólgõ 'schmähen, lästern, beschul-

(89) liv. vörābõz/v(u)orāboz 'Eichhörnchen', digen, tadeln, verachten',

(90) liv. vöržz 'Wipfel, Stange',

(111) liv. pósk 'Wange',

(112) liv. potkāstõ 'treten',

(113) liv. $p \dot{o}$ 'j 'Brust'.

(91) liv. vóz̄̄ 'Teil',

Diese Entwicklung muss aufgrund ihrer hohen Verbreitung ebenfalls älter sein als der Lautwandel $*_{o}>\tilde{o}$. Unter den einschlägigen Belegen hat sich lediglich (30) liv. võttõ 'nehmen' dagegen durchgesetzt. Aufgrund eines einzelnen Belegs lassen sich allerdings kaum Rückschlüsse auf die Entwicklung ziehen und es kann von einem Ausnahmefall ausgegangen werden.

Als letzter Lautwandel, der die Entwicklung zu $\tilde{o}$ in der ersten Silbe verhindert hat, ist für das Livische $*^{*}>u$ vor $m$ zu nennen (PosTI 1942: 10). Er zeigt sich im Korpus in den Belegen (82) liv. u'm 'eigen', (83) liv. umblõ 'nähen' sowie (126) liv. sumār 'Krümel, Krume, Korn'.

\footnotetext{
${ }^{73}$ Da es sich nur um ein Phänomen im Ostlivischen handelt, sei auf die vorhandenen Parallelformen in anderen livischen Dialekten hingewiesen. Einige dieser finden sich auch als Variante, sofern sie anhand der etymologischen Wörterbücher belegt sind, z.B. (89) liv. vórābõz/v(u)orābəz 'Eichhörnchen'.
} 


\section{ABWEICHUNGEN IN DER ERSTEN SILBE}

(51) liv. mūnda 'mancher'

In SSA (II: 171) wird eine Ableitung von einem Pronominalstamm $m o-\sim m u$ - angesetzt. Sofern das Livische von letzterer Form abgeleitet ist, kann dies eine Erklärung für die abweichende Form darstellen.

\section{GEGENPROBE}

Für die Gegenprobe des Livischen wurden die nachfolgend aufgeführten Belege anhand von SSA und EES zusammengestellt. Nicht eingeflossen sind derartige Wörter, deren erste Silbe z.B. einen Lautwandel $* o>$ uo durchlaufen hat.

1 liv. bobīkšõ 'murmeln, brummen' est. pobiseda 'murmeln'

? fi. popottaa 'schnell sprechen, plappern; fiepen (Hase)'

SSA (II: 396), EES (377)

2 ? liv. bomīkš̃̃ 'mit sich selbst reden'

fi. pomista 'laut sprechen; dumpf tönen; murmeln'

SSA (II: 393), (EES 379)

3 liv. jo ' $r$ 'Gebrumme; leeres Geschwätz'

fi. jorista 'undeutlich sprechen, brummen; schwatzen, plaudern'

SSA (I 242), EES (99)

4 liv. klobārtõ 'schütteln, klopfen', klobīntõ 'klappern'

fi. (dial.) klopista 'klingen, klirren'

EES (166); in SSA nicht behandelt

5 liv. ko 'ggõrz 'Karausche (Carassius carassius)'

fi. kouri 'Karausche (Carassius carassius)'

SSA (I: 414); EES (169)

6 liv. ko 'ktõ 'probieren; planen'

fi. kokea 'erleben, durchmachen, prüfen'

SSA (I: 386), EES (169)

ural. koke- 'sehen, besichtigen, finden' (UEW I: 171)

7 liv. kom 'Schlauheit' (LW auch kom̄ 'List, Anschlag' (LW: 144))

fi. komme 'Streich, Trick; Werkzeug'

SSA (I: 394f.), EES (173) 
8 ? liv. lobāz 'Schwätzer' est. lobama 'schwatzen, plappern'

fi. lopista 'schwatzen, quatschen'

SSA (II: 92), EES (244)

9 liv. lol 'dumm'

fi. dial. lolli 'dumm; dick, schlaff, faul'

SSA (II: 90), EES (247)

10 liv. lo 'mmõ 'sich neigen; sich krümmen, sich biegen'

fi. lommo 'Delle'

SSA (II: 90), EES (247)

11 liv. lops 'Ohrfeige'

fi. lopsia 'plappern; essen, schnappen; klopfen, hacken'

SSA (II: 93), EES (251)

12 liv. lorīkšõ 'rieseln; schwatzen'

fi. lorista 'rieseln'

SSA (II: 93f.), EES (251)

13 liv. okš 'Bär'

fi. (Volkslieder) ohto 'Bär; großes Tier oder Mensch'

SSA (II: 260), EES (340)

14 liv. tšolīnțõ 'rieseln, plätschern; hell (er)klingen; unterhalten, quatschen'

fi. solista 'rieseln, plätschern; hell (er)klingen'

SSA (III: 196), EES (479)

15 liv. tšorkõ 'stechen, stochern'

fi. sorkkia 'stechen'

EES (483f.); in SSA nicht behandelt

16 liv. zollk 'Abwasser, Abwaschwasser'

fi. solkata 'durch Tat oder Wort Unordnung, Verwirrung stiften, besudeln; radebrechen, undeutlich sprechen'

SSA (III: 196), EES (479)

1, 2, 8: die Zusammenstellung mit dem fi. Wort gilt als unsicher, der Wortcharakter ist onomatopoetisch und kann so Grund für die Abweichung sein.

3, 4, 7, 9-12, 14-16: Der onomatopoetische bzw. deskriptive Wortcharakter wird für die Abweichung ausschlaggebend sein. 
5, 6: Es konnte nicht ermittelt werden, warum hier keine der Entwicklungen in der ersten Silbe eingetreten ist.

13: Die Verhältnisse sind nicht klar genug, sodass nicht festgestellt werden kann, warum kein Lautwandel eintritt.

Aus dem Korpus sind die nachfolgenden Belege zur Gegenprobe zu ergänzen:

43 liv. loptõ 'beenden'

fi. lopettaa 'beenden'

63 liv. nolgõ 'geifern'

fi. nolki 'Schleim, Geifer'

73 liv. oksnõ 'sich übergeben'

fi. oksentaa '(sich) erbrechen'

87 liv. oppõ '(er)lernen'

fi. oppia '(er)lernen'

123 liv. so'gdo 'blind'

fi. sokea 'blind'

$(43,87)$ Der Lautwandel ${ }^{*} o>$ uo tritt nicht ein, da auf $*^{*} o$ der labiale Konsonant $p$ folgt (PosTI 1942: 8). Ungeklärt ist, warum die Entwicklung $* o>\tilde{o}$ nicht abläuft.

(63) Der Wortcharakter ist deskriptiv (vgl. SSA (II: 229f.)), sodass dies als mögliche Ursache für das Bewahren von $* o$ angesetzt werden kann

(73) Auch hier kann aufgrund des Wortcharakters eine deskriptive Form angesetzt werden, wenngleich sie etymologisch nicht bestätigt ist.

(123) Posti (1942: 8) zufolge liegt eine Entwicklung <*sogu $\delta a<* s o k e \delta a$ vor, deren epenthetisches $u$ der zweiten Silbe eine Entwicklung $*_{o}>$ uo verhindert. Warum kein $\tilde{o}$ auftritt, kann nicht geklärt werden.

Dass die Gegenprobe im Livischen nicht umfangreicher ausgefallen ist, ist u.a. den oben diskutierten eigensprachlichen Entwicklungen zuzuschreiben. Zu diesen sei zu ergänzen, dass der Lautwandel $* o>$ uo unter bestimmten Bedingungen nicht eingetreten ist. POSTI (1942: 8) zufolge komme die Entwicklung nicht vor, wenn auf $o$ ein labialer Konsonant $p$ oder $b$ oder auch $u$ in der zweiten Silbe gefolgt sei, wobei insbesondere der letztere Fall nicht ausnahmslos gelte. Aufgrund der geringen Beleganzahl seien „einige Einzelheiten noch unklar“ (ebd. 9). PosTI 
erklärt damit einen Teil des Wortschatzes, in dem *o bewahrt wurde. Als Folge dessen hätte die Entwicklung $*_{o}>\tilde{o}$ eintreten müssen, wie aber die Gegenprobe verdeutlicht hat, passierte dies nicht.

\subsubsection{Südestnisch}

Die Belege, die sich im Südestnischen dem Lautwandel $* o>\tilde{o}$ in der ersten Silbe zuordnen lassen können, verteilen sich anhand der zweiten Silbe wie folgt:

$\begin{array}{cc}\text { Vokale der zweiten Silbe } & \text { Anzahl } \\ e & 31 \\ a & 10 \\ i & 5 \\ o & 2 \\ \text { unbestimmbar } & 5\end{array}$

Den angegebenen Daten können die angeführten Belege zugeordnet werden: mit zweiter Silbe, die auf * $e$ zurückgeht:

(1) estS. hõpõ 'Silber', (84) estS. õng', gen. ơngõ 'Angel(haken)',

(8) estS. jõgi, gen. jõ õ 'Fluss', (85) estS. ợn', gen. õ $n n \tilde{o}$ 'Glück',

(15) estS. kõllõ 'trocken', (87) estS. .op'ma, II. Infinitiv oppiq '(er)ler(25) estS. .kõrbõma, II. Infinitiv kõrbõdaq nen', kõrvõdaq 'anbrennen', (97) estS. põ|dõma, II. Infinitiv -tõq 'leiden, (27) estS. kõreńd 'Stange', krank sein',

(28) estS. . korgõ 'hoch', (101) estS. .põlgma, II. Infinitiv .põlgõ q'ver(30) estS. kõrs', kõrrõ 'Halm', achten',

(34) estS. kõva 'hart, fest, streng', (104) estS. põlv', gen. põlvõ 'Knie, Generati-

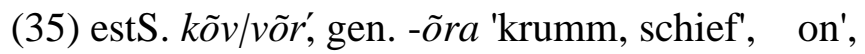
(38) estS. lôhekala; lõh|i, gen. -e 'Lachs', (111) estS. põsk', gen. põso 'Wange', (39) estS. lõhkõ- 'bersten, platzen, sich (123) estS. sõkõ 'dunkel, blind', spalten', (124) estS. sõlg', gen. sõlo 'Spange', (43) estS. .lõpma, II. Infinitiv lõppõq 'been- (125) estS. sõlm', gen. sõlmõ 'Knoten', den', (126) estS. sõm|mõr', gen. -õra 'Kies, grober (49) estS. mõlõmb $\mid a q$ 'beide', Sand', 
(62) estS. nõgi, gen. nõ $\tilde{o}$ 'Ruß',

(63) estS. nõlõ 'Rotz',

(66) estS. .nõtskõ 'biegsam, geschmeidig, den',

weich',

(79) estS. olg', gen. olõ 'Stroh, Halm',

mit zweiter Silbe, die auf $* i$ zurückgeht:

(21) estS. .kõmṕma, II. Infinitiv .kõmpiq;

.kõmpsíma, II. Infinitiv .kõmpsiq 'einher-

schreiten, schwerfällig gehen',

(22) estS. .kõnd'ma, II. Infinitiv .kõndiq 'ge-

hen, spazieren',

mit zweiter Silbe, die auf *a zurückgeht:

(31) estS. kõrv, gen. kõrva 'Ohr',

(54) estS. .mõrsja 'Braut',

(70) estS. .õhkama, II. Infinitiv õhadaq 'seuf-

zen; ausströmen',

(99) estS. põhi, gen. põh'a 'Boden, Grund',

(110) estS. põrss, gen. .põrsa 'Ferkel',

(119) estS. sõba 'Decke, Bettdecke, wollenes

Umschlagetuch',

mit zweiter Silbe, die auf $* o$ zurückgeht:

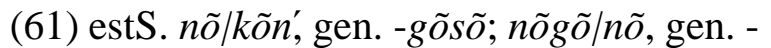

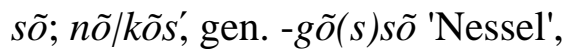

mit zweiter Silbe, die nicht bestimmbar ist:

(10) estS. .jõlkma, II. Infinitiv jõlkuq 'hin und her gehen',

(12) estS. kõt $\mid t$, gen. - $u$ 'Bauch',

(46) estS. mõo|ma, II. Infinitiv -daq 'beein-

flussen, wirken',
(132) estS. sõrm', gen. sõrmõ 'Finger',

(137) estS. tõ $\mid b i$, gen. -võ/bõ 'Krankheit, Lei-

(149) estS. tõtõ 'wahr; Wahrheit';

(128) estS. sõnn, gen. - $i$ 'Schafbock, Widder, Hammel', (129) estS. sõnnik 'Mist, Dünger';

(121) estS. sõda, gen. sõa 'Krieg',

(131) estS. sõrg, gen. sõra 'Klaue',

(135) estS. sõtkas, gen. .sõtka 'Bucephala clangula (Schellente)',

(161) estS. .võtma 'nehmen';

(146) estS. .tõrdo 'Kübel, Kufe';

(136) estS. .sõkma, II. Infinitiv sõkkuq'treten, kneten', (145) estS. tõrjuda 'abwehren, verdrängen'.

Anhand der vorliegenden Belege kann für das Südestnische ein ähnliches Verhältnis konstatiert werden, wie bereits im Rahmen des Estnischen und Wotischen: Ein Lautwandel scheint häufig einzutreten, wenn in der Folgesilbe ein Vordervokal oder $a$ vorliegt. 


\section{ANMERKUNGEN}

$(\mathbf{1 0}, 136)$ Angesetzt werden kann hier wohl, wie auch schon für die standardestnische Form, dass man es bei diesen Verben einerseits um deskriptive bzw. onomatopoetisch beeinflusste Wörter handelt, andererseits auch, dass $-u$ - eine Ableitung ist.

(12) Wie schon im Estnischen scheint der Lautwandel hier unmotiviert, vgl. Kapitel 5.3.3.1. Möglicherweise liegt wechselseitiger Einfluss vor.

(46, 61) Der Grund für den Lautwandel konnte für diese Belege nicht ermittelt werden. Auch hier kann Einfluss des Estnischen vorliegen.

(145) Die Etymologie ist ungesichert, der Grund für die Abweichung daher nicht zu ermitteln, vgl. auch Kapitel 5.3.3.1.

(146) Hier liegt wohl estnischer Einfluss vor. Der dortige Lautwandel wird in der zweiten Silbe durch -e ausgelöst, vgl. est. tõr|s, -re 'Kübel, Kufe'.

Auch im Südestnischen häufen sich Belege, in denen der Lautwandel bei $a$ in der Folgesilbe eintritt. Dabei lässt sich abermals eine Opposition derer aufstellen, in denen ein labialer Laut in direkter Umgebung von $* o$ vorliegt, zu jenen, die den Lautwandel ohne labialen Einfluss vollzogen haben. Die Belege mit labialer Umgebung liegen in gleicher Anzahl vor wie jene ohne, sodass erneut bestätigt werden kann, dass das Labialitätsgesetz nach HoLST kein relevanter Faktor der Lautentwicklung ist: (54), (99), (110), (119), (161). Sie stehen den Belegen ohne Kontakt zu labialen Lauten gegenüber: (31), (70), (121), (131), (135).

Da der Einfluss der labialen Laute nicht ausschlaggebend für die Entwicklung gewesen sein kann, ist auch für das Südestnische festzuhalten, dass diese Belege allesamt Teil des Assimilationsprozesses $* o>\tilde{o}$ sind, hier ausgelöst durch $a$ in der Folgesilbe.

\section{ABWEICHUNGEN IN DER ERSTEN SILBE}

(13) estS. kuht, gen. koha kuha 'Ort, Stelle'

(71) estS. uhi, gen. uha' 'Leine, Zügel'

(82) estS. uma 'eigen' 
(83) estS. .umblõma 'nähen'

$(\mathbf{1 3 , 7 1})$ Eine Entwicklung $*_{o}>u$ vor $h($ EKÄ 132) tritt ein und verhindert bei diesen Belegen den Lautwandel zu $\tilde{o}$ in der ersten Silbe.

$(\mathbf{8 2}, \mathbf{8 3})$ Wie aus dem Livischen bekannt, tritt im Südestnischen ein Lautwandel $*_{o}>u$ vor Nasalen ein (KEEM 2002: 33f.) und verhindert somit den Lautwandel $* o$ o.

\section{GEGENPROBE}

Um die These einer Entwicklung $*_{o}>\tilde{o}$ bei einem Vordervokal oder $a$ in der zweiten Silbe zu untersuchen, soll auch hier die Gegenprobe gemacht werden. Die nachfolgend angeführten Belege konnten dafür im Südestnischen zusammengestellt werden.

1 estS. joris|oma, -taq 'vor sich hinsingen, gröhlen'

fi. jorista 'undeutlich sprechen, brummen; schwatzen, plaudern'

SSA (I 242), EES (99)

2 estS. kogõmaldaq 'unabsichtlich, aus Versehen; zufällig'

fi. kokea 'erleben, durchmachen, prüfen'

SSA (I: 386), EES (169)

ural. koke- 'sehen, besichtigen, finden' (UEW I: 171); est. koge- hier dial.

3 estS. kogõr kokr, gen. kogrõ 'Karausche (Carassius carassius)'

fi. kouri 'Karausche (Carassius carassius)'

SSA (I: 414), EES (169)

$4 \quad$ estS. kohi, kuhi 'impotent; kastriert'

fi. kuoha 'Hoden (besonders des Hengstes)'

SSA (I: 439), EES (169)

5 estS. kolkma, .kolkiq 'klopfen, hauen'

fi. kolkata 'klopfen, poltern'

SSA (I: 390), EES (172)

6 estS. koll', gen. kolli 'Gespenst, Kinderschreck (Folklore)'

fi. (dial.; Volkslieder) koljo 'massiges Wesen (Mensch, Tier); Riese, Krankheit bringender Geist, Teufel; klobiger Gegenstand'

SSA (I: 389f.), EES (173)

fiugr. kolja 'böser Geist' (UEW I: 173) 
7 estS. kollõq, gen. .koldõ 'Teil des Ofens oder Herd, der brennt [...]'

fi. kolle 'unebene Stelle (z.B. einer Wand, Straße); Falte'

SSA (I: 391), EES (173)

8 estS. kombõrdama, kombõrdaq 'stolpern, humpeln'

fi. kompuroida 'stolpern'

SSA (I: 395), EES (173)

8 estS. kommõq, gen. .kombõ 'Brauch'

fi. komme 'Streich, Trick; Werkzeug'

SSA (I: 394f.), EES (173)

10 estS. kopsitama, kopsitaq 'klopfen, pochen'

fi. kopsaa 'klopfen, klappern'

SSA (I: 401), EES (177f.)

11 estS. kos|ima, -siq 'freien'

fi. kosia 'freien'

SSA (I: 408); < urgerm. (EES 179); in LÄGLOS nicht behandelt

12 estS. kott', gen. koti 'Tasche'

fi. kotti 'Beutel; Hodensack; Eihülle (Kuh, Pferd), Gebärmutter, Bauch; Hülle, (Samen)gehäuse; Fischgefäß, kleines Gefäß, Trog (auch als Schleife zum Mistfahren)' urfi. *kotti (LÄGLOS II: 108)

< germ. (SSA I: 413); < urgerm. (EES 180); germ. Lehnwort (LÄGLOS 108f.)

fi.-perm. konte 'Korb, Ranzen aus Birkenrinde' (UEW I: 177)

13 estS. nolk, gen. nolgi 'Jüngelchen, zu großer Mann; junges Tier; nicht ausgewachsener untermaßiger Fisch'

fi. nolkki Bursche, Bengel'

SSA (II: 230), EES (316)

14 estS. pobis|õma, -taq 'murmeln' est. pobiseda id.

? fi. popottaa 'schnell sprechen, plappern; fiepen (Hase)'

SSA (II: 396), EES (377)

15 estS. pomis|õma, -taq 'murmeln, brummen'

fi. pomista 'laut sprechen; dumpf tönen; murmeln'

SSA (II: 393), EES (379) 
16 estS. rops'ma, .ropsiq 'schlagen, zappeln; schütteln; klopfen'

fi. ropsia '(leicht mit einem Bund grüner Birkenzweige) schlagen; fegen, wedeln;

schlendern; pfuschen;'.

SSA (III: 93), EES (437)

17 estS. sori|tsõma, -tsaq -daq 'verleumden, klatschen, nörgeln'

fi. sorista 'brodeln, rieslen; eifrig, durcheinanderreden; knistern, knattern, rasseln; summen'

SSA (III: 201f.), EES (483)

18 estS. .tśokśma, .tśoksiq 'hämmern'

fi. toksahtaa 'plötzlich auftauchen; (gegen etwas) stoßen; etwas festhalten' EES (536), in SSA nicht behandelt

19 estS. tsoliseda 'rieseln, plätschern; hell (er)klingen' (SSA und EES)

fi. solista 'rieseln, plätschern; hell (er)klingen'

SSA (III: 196), EES (479)

20 estS. tsolk 'Spülwasser'

fi. solkata 'durch Tat oder Wort Unordnung, Verwirrung stiften, besudeln; radebrechen, undeutlich sprechen'

SSA (III: 196), EES (479)

21 estS. tsongi. 'wühlen, (durch)wühlen, graben '

fi. sonkia '(durch)wühlen, graben'

SSA (III: 198), EES (480)

22 estS. tsopp 'Ende, Winkel'

fi. soppi 'Ecke, Winkel'

SSA (III: 200f.), EES (483)

23 estS. $\operatorname{tsorkam}(m) a$ 'stechen, hineingießen'

fi. sorkkia 'stechen'

EES (483f.), in SSA nicht behandelt

1, 5, 8-10, 13-21, 23: Die Abweichung ist wohl dem onomatopoetischen bzw. deskriptiven Wortcharakter geschuldet.

2: Die standardestnische est. kogemata sei lexikalisiert (EES 169). Ob Einfluss auf das Südestnische vorliegt, ist ungewiss und etymologisch nicht bestätigt. 
3: Es kann nicht aufgeklärt werden, warum der Lautwandel hier nicht eintritt, vgl. auch die est. Entsprechung in Kapitel 5.3.3.1.

4: Eine Kürzung des Langvokals vor intervokalischem $h$ liegt wohl vor, wenngleich anzumerken ist, dass diese im Südestnischen nicht immer einzutreten scheint (EKÄ 98). Ein Lautwandel *oo $>\tilde{o} \tilde{o}$ tritt nicht ein. Für die Variante estS. kuhi gilt $o>u$ vor $h$ (EKÄ 132).

6: Wie schon bei der Gegenprobe zum Estnischen angeführt, wird ein fiugr. Stamm mit hintervokalischem Auslaut angesetzt, der für den Erhalt von $* o$ ausschlaggebend gewesen sein kann.

7, 11, 12, 22: Die Entwicklung ist ungeklärt.

Ferner können aus dem Korpus die folgenden Belege für eine Gegenprobe genannt werden:

17 estS. kolm, gen. kolmõ 'drei'

fi. kolme 'drei'

28 estS. .korgõ 'hoch'

fi. korkea 'hoch'

73 estS. ossõnda|ma, II. Infinitiv - $q$ 'sich übergeben'

fi. oksentaa '(sich) erbrechen'

79 estS. olg', gen. olõ 'Stroh, Halm'

fi. olki 'Stroh, Halm'

81 estS. oluq, gen. ollõ 'Bier'

fi. olut 'Bier'

urfi. *olut (LÄGLOS II: 310)

87 estS. .op'ma, II. Infinitiv oppiq '(er)lernen'

fi. oppia '(er)lernen'

90 estS. ors', gen. orrõ 'Stange, Hühnerstange'

fi. orsi 'Balken; Hühnerstange'

94 estS. .ots'ma, II. Infinitiv .otsiq 'suchen'

fi. etsiä 'suchen'

urfi. *ecci- / *occi- (LÄGLOS I: 58)

114 estS. .rohkõ 'reichlich'

fi. rohkea 'mutig, kühn; (als Mengenangabe) viel, reichlich'

urfi. *rohke- $\delta a$ (LÄGLOS III: 167)

130 estS. sorrõ 'undicht, grob'

fi. sorea 'ebenmäßig, schön' 
(17) Zahlwörter haben eine hohe Gebrauchshäufigkeit, sodass dieses Kriterium für eine Abweichung des Belegs zugrunde gelegt werden kann.

$(\mathbf{2 8}, \mathbf{7 9}, \mathbf{8 7}, \mathbf{9 0}, \mathbf{9 4}, \mathbf{1 1 4}, \mathbf{1 3 0}, \mathbf{1 3 9})$ Ein Grund für die Abweichung konnte nicht festgestellt werden.

(73) Hierbei handelt es sich wohl um ein deskriptives Wort, sodass dies die Ursache für die abweichende Form der ersten Silbe sein kann.

(81) Vgl. die Diskussion zu est. ôlut 'Bier' in Kapitel 5.3.3.1. Das $u$ der Nominativform scheint im Südestnischen dominant zu sein und dafür zu sorgen, dass die Entwicklung $*_{o}>\tilde{o}$ nicht eintritt.

Auffällig ist, dass der Grund für eine Abweichung der Annahme, $*^{*}>\tilde{o}$ trete bei vordervokalischer Folgesilbe ein, meist nicht genauer bestimmt werden kann. Schon KEEM (2002: 33) notiert, dass in den südestnischen Dialekten andere Verhältnisse vorliegen als in den standardestnischen Entsprechungen, führt allerdings keine weiteren Details aus, sodass die Bedingungen unklar bleiben. Etymologisch wird das Südestnische meist nicht behandelt, daher kann auch nur selten gesichert auf Fremdeinfluss geschlossen werden. Aufgrund der dargelegten Gegenprobe anzunehmen, dass $\tilde{o}$ im Anlaut nicht eintritt, ist naheliegend, doch gibt es eine ausreichende hohe Anzahl an Belegen, die diese Vermutung widerlegen, z.B. (79) und (84). Feststeht trotz einer Vielzahl an Unklarheiten, dass der Lautwandel $*^{o}>\tilde{o}$ in hohem Maße bei einem Vordervokal oder $a$ in zweiter Silbe eintritt.

\section{$5.4 \quad$ Langvokale}

Die Langvokale stellen in dieser Betrachtung die kleinste der drei Kategorien dar. Insgesamt besteht dieser Teil des Korpus nur aus 15 einschlägigen Belegen. Im Estnischen sind sie alle einschlägig, im Südestnischen 13, im Wotischen zehn von ihnen, im Livischen sogar elf. Trotz geringer Gesamtanzahl kann das Verhältnis als relativ ausgewogen angesehen werden. 


\subsubsection{Statistische Auswertung}

Im Korpus gibt es in den untersuchten Sprachen keine Belege, die einem Lautwandel zu $\tilde{o} \tilde{o}$ von *oo ausgehend unterliegen, was bedeutet, dass Wörter mit ursprünglich langem *oo nicht davon erfasst wurden, vgl. z.B. est. soo 'Sumpf' $\sim$ wot. sō id. liv. sūo id. $\sim$ estS. suu id. ${ }^{74}$ Gleiches gilt auch für andere Vokale in der ersten Silbe: einschlägige Belege, die auf andere Laute als *ee zurückgehen, konnten bei der Zusammenstellung nicht ermittelt werden. In Einzelfällen konnte die erste Silbe nicht sicher nachvollzogen werden. Eine Verteilung der einschlägigen Belege gestaltet sich in den drei Sprachen wie folgt: ${ }^{75}$

\section{Silbe *ee 1. Silbe unbekannt}

$\begin{array}{lcc}\text { Estnisch } & 13 & 2 \\ \text { Wotisch } & 10 & \text { ohne } \\ \text { Livisch } & 9 & 2 \\ \text { Südestnisch } & 12 & 1\end{array}$

\subsection{2 *ee> $\tilde{o} \tilde{O}$}

Die Langvokalbelege durchlaufen den Lautwandel *ee > $\tilde{o}$, wenn ein Hintervokal in der Folgesilbe vorliegt. Sie verteilen sich in den drei Sprachen, wie nachfolgend dargestellt:

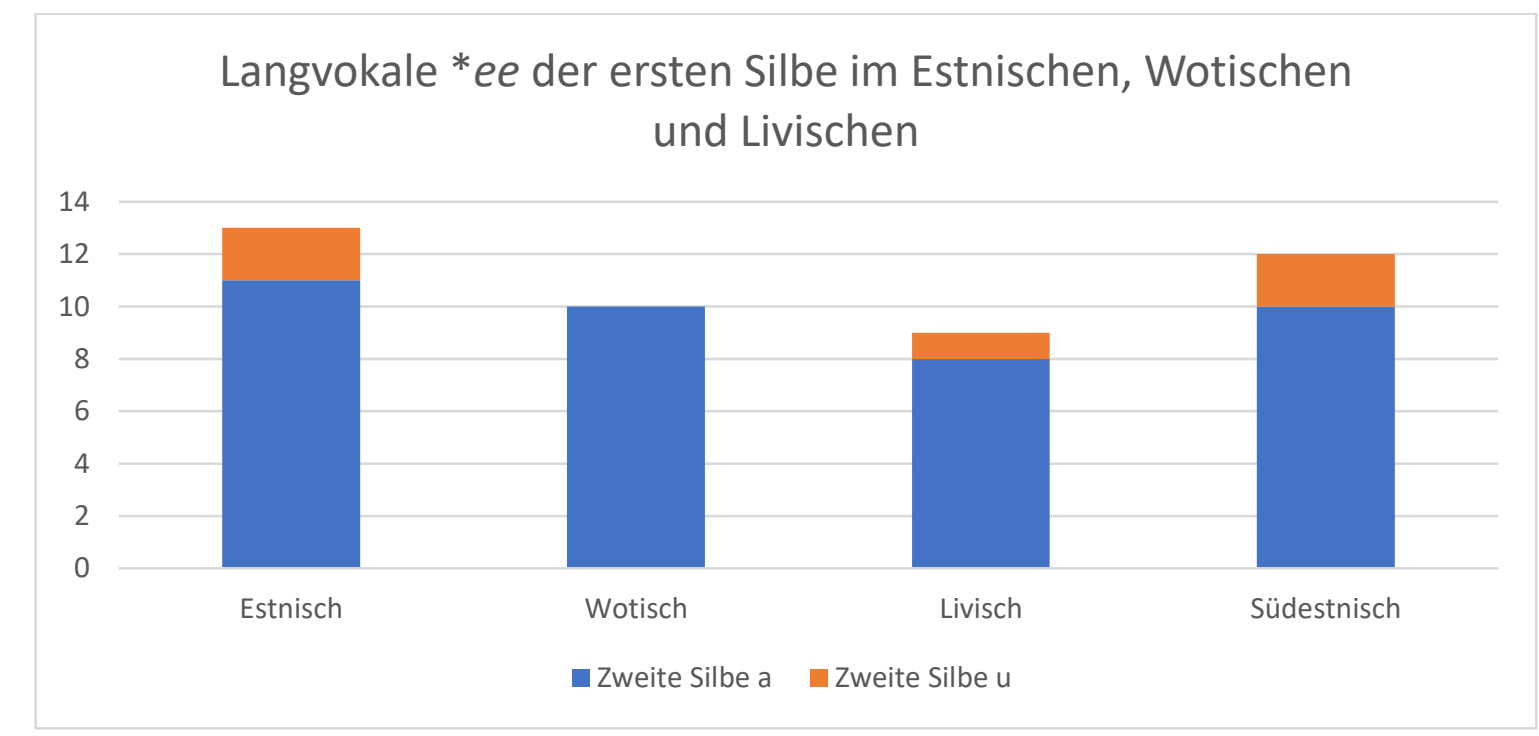

\footnotetext{
${ }^{74} \mathrm{Zu}$ den Entwicklungen der livischen und südestnischen Entsprechungen, s. LAANEST (1982: 128) und EKÄ (138).

${ }^{75}$ Für Belege, deren erste Silbe unbekannt ist, vgl. Kapitel 5.7.
} 


\subsubsection{Estnisch}

Bei den Entwicklungen von *ee sind die Laute in der zweiten Silbe der estnischen Belege allesamt auf einen hintervokalische Folgesilbe $* a$ (elf Belege) oder $u$ (zwei Belege) zurückzuführen:

mit zweiter Silbe, die auf $* a$ zurückgeht:

(163) est. hõ̃ruda '(ver)reiben, scheuern', (170) est. $\tilde{o} h v(a)$ 'Sterke, Färse',

(165) est. lõogg, gen. lõa 'Leine', (171) est. põ̃on, gen. - $a$ 'Querleiste',

(166) est. lõõsk, gen. - $a$ 'große Flamme', (172) est. rõ̃os $\mid k$, gen. - $a$ 'frisch, süß, unge(167) est. lõ̃ts, gen. - a 'Blasebalg', säuert',

(168) est. $m \tilde{o} \tilde{o} \mid k$, gen. - $g a$ 'Schwert', (175) est. sõ̃or|e, gen. -me 'Nasenloch, Nüs-

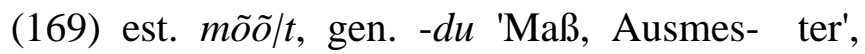
sung', (176) est. võ̃ras 'fremd, unbekannt, Gast';

mit zweiter Silbe, die auf $* u$ zurückgeht:

(173) est. rõ̃m, gen. - $u$ 'Freude, Vergnügen', (174) est. sõ̃m, gen. - $u$ 'Schluck, Zug'.

\section{ANMERKUNGEN}

(163) Das $u$ ist ein Ableitungssuffix (KASIK 2015: 165ff.). Der ursprüngliche Wortstamm lautete in der zweiten Silbe wohl auf $a$, vgl. fi.-perm. šera ( $\check{s} \bar{e} r a)$ 'Schleifstein, Wetzstein; schleifen, wetzen' (UEW II: 783).

(170) Ein ursprünglicher Langvokal ist vor dem Hintergrund weiterer osfi. Entsprechungen wohl ansetzbar, fi. hieho 'Färse, Kuhkalb' ing. hēvikke id. (SSA I: 160). Womöglich hat man es mit einer analogen Entwicklung dieses Belegs zu der Kürzung des Langvokals vor intervokalischem $h$ zu tun, vgl. est. maha <*māhan 'herab, herunter' (EKÄ 98). Ob zunächst die Kürzung oder der Lautwandel *ee > $\tilde{o} \tilde{o}$ eingetreten ist, kann nicht genauer bestimmt werden und ist für das Ergebnis an dieser Stelle unerheblich, da beide möglichen Entwicklungen zur gleichen Form $\tilde{o} h v(a)$ geführt hätten.

(169) $-u$ ist hier wohl als Ableitung ansetzbar, der rekonstruierte Urstamm ist urfi. *mētta(LÄGLOS II: 280). 
(175) Das - $e$ (gen. -me) ist hier ein Ableitungssuffix (EKG 534ff.; KASIK 2015: 272ff.). In einer frühostseefinnischen Form muss die zweite Wortsilbe hintervokalisch gewesen sein, wie die Entsprechungen im Finnischen und Samischen verdeutlichen: fi. sierain 'Nasenloch, Nüster' lpN. suorram id. Wahrscheinlich ist folglich, dass $a$ zunächst bewahrt und erst nach Ablauf des Lautwandels synkopiert wurde. Somit ist auch HoLSTS (2001: 89) Theorie, dass das *a der zweiten Silbe zum Zeitpunkt des Aufkommens von $\tilde{o}$ noch vorhanden gewesen sei und den Lautwandel ausgelöst habe, als plausibel zu bewerten.

\section{GEGENPROBE}

Für einen Gegenbeweis konnten die nachfolgend angeführten Belege anhand von SSA und EES zusammengestellt werden. Im Korpus dieser Untersuchung konnten keine weiteren Belege festgestellt werden, in denen *ee im Estnischen in der ersten Silbe erhalten bleibt.

1 est. keeda 'kochen'

fi. kiehua 'sieden'

SSA (I: 352), EES (140f.)

fiugr. (? ural.) keje- '(intr.) kochen, gekocht, gar, reif werden' (UEW I: 143f.)

2 est. keerata 'drehen; kehren, wenden'

fi. kiertää 'drehen, umdrehen; umwickeln; [...]'

SSA (I: 355), EES (141)

fiugr. kerä 'rund, rollend; sich drehen, drehen, wenden' (UEW I: 147)

3 est. leelo, gen. leelo 'Leelo (Form von Volkslied aus Südostestland)' estS. leelo id. EES (233), in SSA nicht behandelt

$4 \quad$ est. needa 'fluchen'

fi. nietää 'fluchen'

SSA (II: 217), EES (309)

5 est. neelata 'schlucken, verschlingen'

fi. niellä '(ver)schlucken'

SSA (II: 216), EES (309)

ural. ńele- (ńēle-) '(ver)schlucken, (ver)schlingen' (UEW I: 315)

$6 \quad$ est. peenar, gen. peenra 'Beet, Feldrain'

fi. piennar 'Rain, Graben-, Wegrand'

SSA (II 349), EES (359) 
1: Es wird vom gleichen Ursprung ausgegangen wie fi. keittää 'kochen' (SSA I: 352), sodass Vokalharmonie und somit eine vordervokalische Folgesilbe als alt angesetzt werden können.

2, 4, 5: Die Belege zeigen insbesondere im direkten Vergleich mit den finnischen Entsprechungen, dass die zweite Silbe historisch vordervokalisch gewesen ist. Der Abbau der Vokalharmonie führte zur Abweichung im Estnischen.

3: Der onomatopoetische Charakter des Wortes ist wohl ausschlaggebend dafür, dass weiterhin *ee vorliegt.

6: Der Erhalt von *ee kann nicht begründet werden.

Die Gruppe der Gegenbeweise ist recht klein und deutet darauf hin, dass der Lautwandel *ee > $\tilde{o} \tilde{o}$ bei hintervokalischer zweiter Silbe im Estnischen wohl regelmäßig ist. Die meisten Belege der Gegenprobe sind aufgrund der fehlenden Vokalharmonie im Estnischen abweichend, lediglich ein Beleg ist fraglich. Dass die aus den einschlägigen Belegen geschlossene Annahme eines Lautwandels bei Hintervokal richtig sein muss und weitere Laute in Form eines Langvokals nicht erfasst werden, lässt sich anhand einiger Vorarbeiten stützen. WIIK (1986: 40f.) weist darauf hin, dass man die Erklärung für historisch *ee aufgrund der Kurzvokal-Regel nicht weiter erwähnen müsse, was eine Regelmäßigkeit bei einer hintervokalischen zweiten Silbe impliziert. Auch HoLST (2001: 79) notiert, dass sich das lange *ee wie das kurze verhalte, woraus sich das gleiche Kriterium für die Entwicklung ergibt. Einen Lautwandel *oo $>\tilde{o} \tilde{o}$ halten beide für ausgeschlossen (WIIK 1986: 42; HOLST 2001: 79). Weitere Bedingungen werden nicht festgelegt, da es keine anderen Laute gibt, die in langer Vertretung einem Lautwandel unterliegen.

\subsubsection{Wotisch}

Im Wotischen könne alle zehn Belege in der zweiten Silbe auf den Hintervokal $a$ zurückgeführt werden:

(163) wot. õortaa '(ver)reiben, scheuern',

(169) wot. mõõtto 'Maß',

(165) wot. lõõka 'Strick, Kette', (170) wot. ôhva 'Sterke, Färse',

(166) wot. lõõska 'Feuerzug, Heizkanal',

(172) wot. rõ̃oska 'frisch, süß, ungesäuert',

(167) wot. lõ̃tsua 'Bälge treten',

(175) wot. sẹer (a)med 'Nasenloch, Nüster',

(168) wot. mõ̃okka 'Schwert',

(176) wot. võ̃raz 'fremd, unbekannt, Gast'. 


\section{ANMERKUNGEN}

(167) Der Beleg geht auf urfi. *lēcca zurück (LÄGLOS II: 201), sodass ein *a in der zweiten Silbe als ursprünglich anzusetzen ist. Das - $u$ - ist hier Ableitungssuffix (vgl. ARISTE 1968: 119).

(169) Das -o ist ein Ableitungssuffix (vgl. ARISTE 1968: 115). Als Urstamm wird urfi. *mēttaangesetzt (LÄGLOS II: 280), sodass auch hier * $a$ als ursprünglicher Vokal gilt.

(170) Vgl. die Diskussion der est. Entsprechung in Kapitel 5.4.2.1.

(175) Als primäre Vertretung ist eine Pluralform mit hintervokalischer zweiter Silbe anzusetzen, da dieses Wort am häufigsten im Plural in Gebrauch gewesen zu sein scheint. Es existiert noch eine Singularvariante, wot. sõ̃rmi, bei der eine sekundäre Herausbildung angenommen werden kann. Fraglich ist, ob es sich hierbei nicht sogar um eine von AHLQVIST geschaffene Rekonstruktion handelt. Sie findet sich nur in seiner Aufzeichnung, weitere werden laut VKS üblicherweise im Plural verwendet (vgl. VKS 1208). Einen Beweis für einer derartige Theorie gibt es jedoch nicht. Singularformen werden in SSA (III: 173) nicht angeführt und auch in EES (497) geht man von einer üblicherweise pluralischen Verwendung aus.

\section{ABWEICHUNGEN IN DER ERSTEN SILBE}

(171) wot. piina 'Pfosten Pfeiler'

Hierbei handelt es sich um karelischen Einfluss, der für eine Entwicklung *ee > ii sorgte (VKÄ 131).

Zur Gegenprobe konnte im Wotischen aus SSA und EES lediglich ein Beleg festgestellt werden. Anhand des Korpus konnten keine Ergänzungen identifiziert werden.

1 wot. vēno 'sanft abfallen, senkend; langsam'

fi. vieno 'zart, schwach, mild, lau; weich (Schneide, Klinge) '

SSA (III: 433), in EES nicht behandelt

1: In VKS wird dieser Beleg als Variante von wot. võono 'langsam; flach, ruhig; lauwarm' angesehen, welches etymologisch nicht behandelt wird. Eine Zusammenstellung scheint vor dem Hintergrund des Lautwandels *ee > $\tilde{o} \tilde{o}$ durchaus plausibel, warum dieser aber in der Variante, 
in der *ee bewahrt bleibt, nicht einritt, kann nicht begründet werden, da keine Etymologie vorliegt. Womöglich liegt Fremdeinfluss oder eine (dialektale) Variante vor.

Da für die Gegenprobe nur ein etymologisch bestätigter Beleg gefunden wurde, bei dem es sich womöglich um eine Variante handelt, kann angesetzt werden, dass es sich im Wotischen bei $*_{e e}>\tilde{o} \tilde{o}$ vor hintervokalischer Folgesilbe wohl um eine regelmäßige Entwicklung handelt.

\subsubsection{Livisch}

Insgesamt gehen neun Wörter im Livischen auf *ee zurück. Sie verteilen sich wie nachfolgend anhand der Vokale der zweiten Silbe:

mit zweiter Silbe, die auf * $a$ zurückgeführt werden kann:

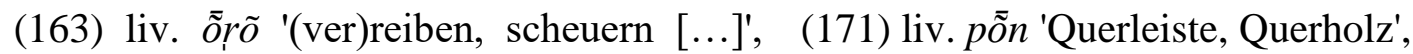

(167) liv. lõ̃tstõ 'keuchen, schnaufen, [...]', ， (172) liv. rõ̃skõ 'frisch, süß, ungesäuert',

(168) liv. $m \overline{\tilde{k}} k$ 'Schwert',

(175) liv. sõ̃rmõz 'Nasenloch, Nüster',

(170) liv. $\tilde{o}^{\prime} u v$ 'Sterke, Färse, junge Kuh (vor (176) liv. võ̃rõz 'fremd, unbekannt, Gast';

dem Kalben)',

mit zweiter Silbe, die auf $* u$ zurückgeführt werden kann:

(173) liv. rõ̃m 'Freude'.

\section{ANMERKUNGEN}

(170) Posti (1942: 245) geht von einem *o als Vokal der zweiten Silbe aus und setzt für das Livische folgende Entwicklung mit Metathese an: *(h)êhvo $>*$ equho $>*$ evu $>$ ěcuv. Die erste Silbe muss ursprünglich wohl lang gewesen sein (vgl. die Diskussion um die est. und wot. Belege). Der Vokal der zweiten Silbe ist nicht genau zu ermitteln, da die Herkunft des Wortes unklar ist. Betrachtet man die Laute dieser Position in anderen ostseefinnischen Sprachen, so kann ein Hintervokal, wohl $a$, angesetzt werden: est. $\tilde{o} h v(a)$ 'Sterke, Färse' wot. õhva id. estS. (h)ôhv, gen. (h)õhva id.

(175) Wie schon in den vorausgegangenen Kapiteln angemerkt, war die zweite Silbe dieses Belegs in einer frühostseefinnischen Form zunächst hintervokalisch. Das $a$ wurde im Livischen synkopiert. Im Nominativ Singular ist $-z$ im Livischen ein übliches Merkmal (LW XXXIX). Vgl. ferner die Diskussion in den Kapiteln 5.4.2.1 und 5.4.2.2. 


\section{GEGENPROBE}

Für die Gegenprobe konnten für das Livische lediglich ein Beleg im Korpus festgestellt werden.

Dies ist v.a. den innersprachlichen Entwicklungen geschuldet, die das Aufkommen von $\tilde{o}$ verhindert haben und die auch die Langvokale betreffen, vgl. hier ${ }^{*} \bar{e}>$ ie (POSTI 1942: 27f.).

174 liv. sēmda 'Milch'

fi. siemaista 'geirig oder schnell trinken, hinunterkippen, -schlürfen'

(174) KETTUNEN (LW 358f.) diskutiert verschiedene Optionen: Es könne ein deskriptives Wort sein und mehrere Varianten haben, aus der Kindersprache stammen und daher keine Etymologie haben oder gar aus dem Lettischen entlehnt sein (vgl. lett. (Dunika) zēmilža 'von Kühen' und lett. semilše 'eine Kuh, die das 2. Jahr gemolken wird').

Für das Livische lässt sich eine regelmäßige Entwicklung ebenso bestätigen wie für die zuvor diskutierten Sprachen. Die Gegenprobe hat nicht keine Belege ergeben, die die Annahme eines regelmäßigen Lautwandels *ee > $\tilde{o}$ bei hintervokalischer Folgesilbe widerlegen.

\subsubsection{Südestnisch}

Die nachfolgenden Belege sind im Südestnischen für die Entwicklung *ee > $\tilde{o} \tilde{o}$ einschlägig. mit zweiter Silbe, die auf $* a$ zurückgeht:

(163) estS. hõ õr $(d) m a$, II. Infinitiv hõ õ r(d)uq (171) estS. põõn, gen. põona 'Querleiste', '(ver)reiben, scheuern [...]', (172) estS. rõõosk, gen. rõ̃sa 'frisch, süß, un-

(165) estS. lốig, gen. lốia 'Leine', gesäuert',

(167) estS. lõõts, gen. lõõdsa 'Blasebalg', (175) estS. sõõrmas, gen. sõ õrma; sõorrmõs, (168) estS. mõ õ k, gen. mõoga 'Schwert',

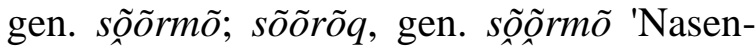
(169) estS. mõ ôt, gen. mõ õdu 'Maß, Ausmesloch, Nüster', sung', (176) estS. võoras, gen. võ õ ra 'fremd, unbe-

(170) estS. (h)õhv, gen. (h)õhva 'Sterke, kannt, Gast';

Färse', mit zweiter Silbe, die auf * $u$ zurückgeht: (173) estS. rõ õ̃m, gen. rõ̃mu 'Freude, Ver(174) estS. sõ̃õm, gen. sõ̃mu 'Schluck, gnügen', Zug'. 


\section{ANMERKUNGEN}

(165) KetTUNEN (EKÄ 134) setzt für das Standardestnische in der alten Schriftsprache ein Verhältnis $\tilde{o} \tilde{o} \sim \tilde{o} e$ an. Fraglich ist, ob es derartige Formen analog auch im Südestnischen gab und dabei auch eine Entsprechung $\tilde{o} \tilde{o} \sim \tilde{o} i$ zustande kam. Weder KETTUNEN noch KEEM stellen Überlegungen dazu an, vergleichbare Erscheinungen konnten anhand der Wörterbücher nicht ermittelt werden, sodass es wohl als unmotivierte Entwicklung anzusetzen ist.

(170) Vgl. die Diskussion um die est. Entsprechung in Kapitel 5.4.2.1.

(175) Im Südestnischen ist - $m$ - ebenfalls Bestandteil einer Ableitung. Die Entwicklung des Belegs ist wohl als ähnlich wie im Estnischen ansetzbar, vgl. Kapitel 5.4.2.1.

\section{GEGENPROBE}

Für die Gegenprobe zu dieser Lautentwicklung konnten für das Südestnische die nachfolgend angeführten Belege zusammengestellt werden. Anhand des Korpus konnten keine Ergänzungen identifiziert werden.

1 estS. keero|tama, -taq, drehen, kreisen

fi. kiertää 'drehen, umdrehen; umwickeln; [...]'

SSA (I: 355), EES (141);

fiugr. kerä 'rund, rollend; sich drehen, drehen, wenden' (UEW I: 147)

2 estS. leelo 'Leelo (Form von Volkslied aus Südost-Estland)' est. leelo, gen. leelo id.

EES (233), in SSA nicht behandelt

1: -o- muss wohl eine Ableitung sein, vgl. auch est. keerutama 'drehen, kreisen'.

2: Der onomatopoetische Charakter des Wortes ist für die Abweichung wohl ausschlaggebend.

Der Gegenbeweis konnte die These einer wohl regelmäßigen Entwicklung *ee $>\tilde{o} \tilde{o}$ bei einem Hintervokal in der zweiten Silbe im Südestnischen nicht entkräften, soweit die knappe Anzahl der Belege Schlüsse zulässt. Die Ergebnisse entsprechen somit denen der anderen Sprachen. 


\subsubsection{Statistische Auswertung}

In dieser Untersuchung sind 45 Belege für die Kategorie der Diphthonge einschlägig. Auf das Estnische entfallen 37 dieser Belege, im Wotischen sind es 30, im Südestnischen 30 und abermals bildet das Livische mit lediglich 19 Belegen die kleinste Gruppe. Die Diphthonge zerfallen dabei auf eine Vielzahl an Ausgangslauten und sorgen somit für ein sehr heterogenes Gesamtbild.

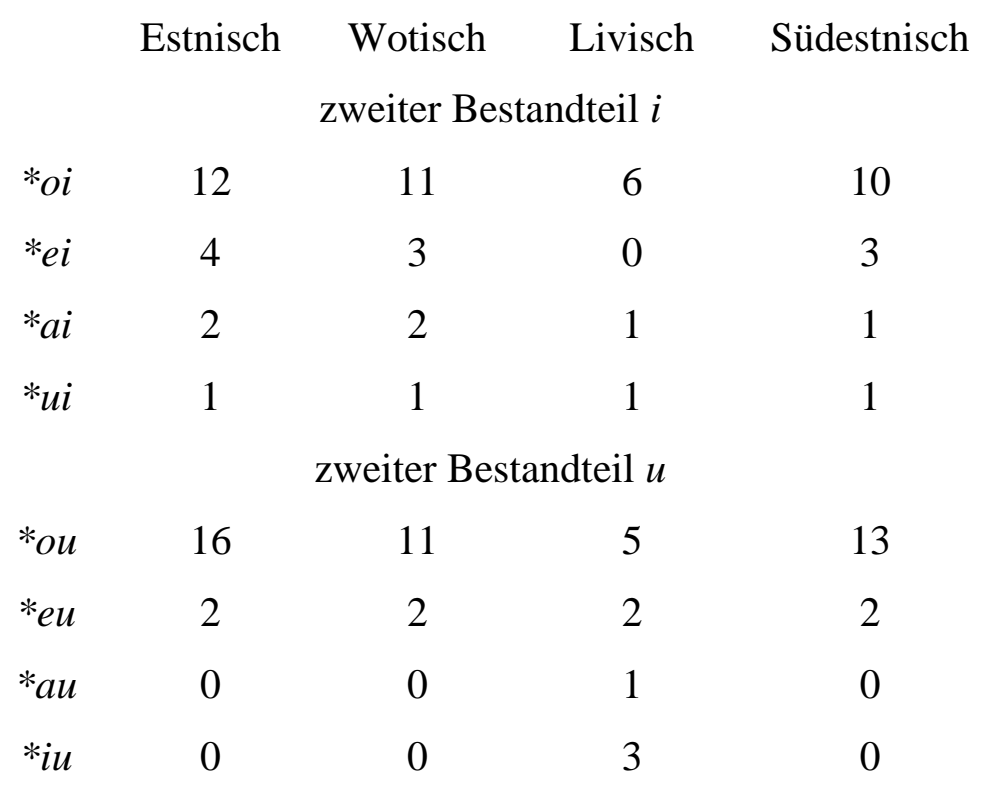

Zwei starke Vertretungen stechen anhand der Übersicht hervor: Die Diphthonge, deren erster Bestandteil *o ist, sind für den Lautwandel scheinbar in allen Sprachen besonders empfänglich. Deutlich wird auch, dass nur Diphthonge einen Lautwandel vollzogen haben, deren zweiter Bestandteil $i$ oder $u$ ist. Liegt in der heutigen Wortform ein anderer Laut in zweiter Position vor, ist der erste Bestandteil in diesem Kontext nicht einschlägig.

\subsubsection{Diphthonge mit $u$ als zweitem Bestandteil}

Als Diphthonge, deren zweiter Bestandteil $u$ lautet und deren erster eine Entwicklung zu $\tilde{o}$ durchläuft, konnten in diesem Korpus *ou, *eu, *au und *iu identifiziert werden. Die letzteren beiden sind ausschließlich im Livischen einschlägig und treten somit nur in geringer Anzahl 
auf. $^{76}$ Die größte Gruppe unter diesen Diphthongen bildet jene, die dem Lautwandel $* o u>\tilde{o u}$ unterliegt.

\subsubsection{1 *ou $>\tilde{o u}$}

Für den Lautwandel $* o u>\tilde{o} u$ sind im Estnischen sind insgesamt 16 Belege einschlägig, im Südestnischen 13, im Wotischen sind es elf und das Livische verzeichnet fünf Wörter. Über die Verteilung der auf*ou zurïckgehenden Belege gibt das nachfolgende Diagramm Aufschluss. Abermals zeigt sich deutlich, dass die größte Vertretung der Vokale der zweiten Silbe * $a$ lautet. Eine starke Vertretung liegt noch bei $* o$ vor, alle anderen Laute treten in den Folgesilben nur noch marginal auf. Das Bild ist also wenig homogen, wenngleich die Hintervokale dominieren.

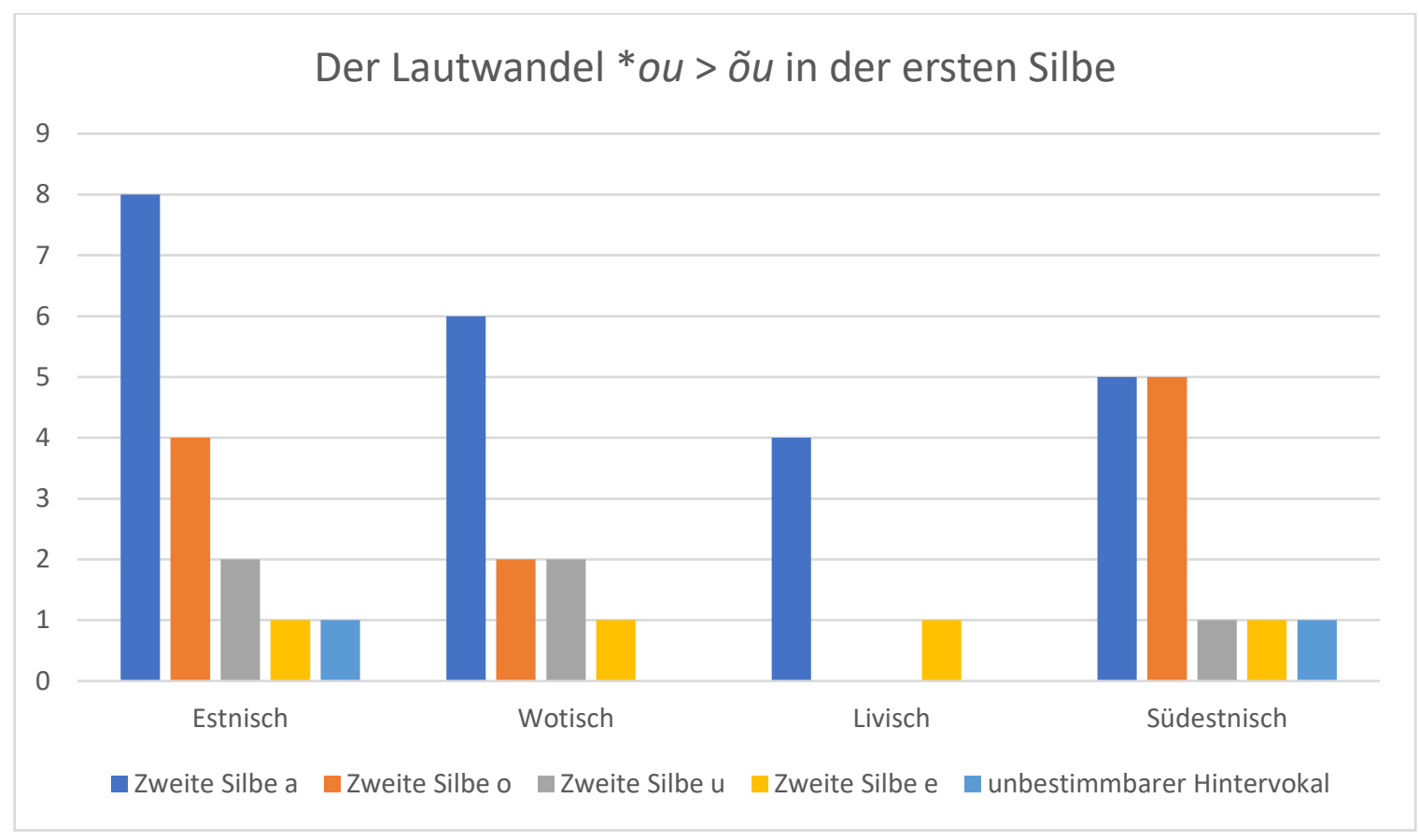

\subsection{Estnisch}

Betrachtet man zunächst das Estnische, so lassen sich für dem Lautwandel $*$ ou $>\tilde{o u}$ die nachfolgenden Belege zuordnen.

mit zweiter Silbe, die auf *a zurückgeht:

(178) est. jõ $h v$, gen. $-i,-e$ 'Pferdehaar, Rosshaar',

\footnotetext{
${ }^{76}$ Die Diphthonge *au und *iu werden hier nicht näher behandelt. Vgl. dazu Kapitel 5.7 Entwicklungen mit marginaler Vertretung.
} 
(179) est. jõuda 'können, vermögen; Zeit haben',

(190) est. lõu|gas, gen. - $k a$ 'gemauerte Seite des Aschenloches vor der Ofenmündung

(als Sitz dienend), Ecke, Krümmung, Meerbusen',

(192) est. lõuna 'Süd, Mittag, Mittagessen',

(198) est. nõuda 'verlangen, fordern',

(204) est. põu|d, gen. - $a$ 'Dürre',

(212) est. sõuda 'rudern',

(215) est. tõugata 'stoßen, schieben',

(216) est. tõu|k, gen. - gu 'Larve, Wurm';

mit zweiter Silbe, die auf *u zurückgeht:

(182) est. jõulud 'Weihnachten',

(207) est. rõu|k, gen. - gu 'Stütze des Wagenleiters';

mit zweiter Silbe, die auf $* o$ zurückgeht:

(181) est. jõu|k, gen. - $g u$ 'Bande, Rotte, Horde',

(201) est. õud(ne) 'unheimlich',

(206) est. rõu|k, gen. - gu '(auf Reitern, Reutern aufgeschichteter) Klee- oder Kornschober',

(214) est. tõug, gen. tõu 'Sommersaat, -korn';

mit unbestimmbarem Hintervokal:

(186) est. kõu, gen. -e 'Donner'.

\section{ANMERKUNGEN}

(178) Zunächst setzt eine Metathese ein, dann *u>v: -uhh- > (-hu-) - hv- (EKÄ 98). Für den Stammvokal liegen im Estnischen laut SSA (I: 243) Varianten auf $-i$ und auf $-e$ vor, wobei $-e$ wohl alt ist (vgl. fi. jouhi, gen. jouhen 'Rosshaar' oder weps. jovez id., jouhed id.-NOMPL).

(186) Eine indoeur. oder balt. Herkunft des Stammes wird in den etymologischen Wörterbüchern diskutiert, Einigkeit herrscht in dieser Frage nicht (vgl. SSA (I: 414) und EES (203)). Da die lehngebenden Formen in beiden Fällen hintervokalisch sind, kann ein Hintervokal als historische Vertretung des Stammvokals angesetzt werden, wenngleich er unbestimmbar ist. 
(201) -ne ist ein Ableitungssuffix (KASIK 2015: 348). Der Wortstamm ist etymologisch nicht gesichert, die finnischen und karelischen Entsprechungen fi. outo 'fremd, unbekannt, ungewöhnlich [...]' kar. outo id. (SSA II: 277) deuten auf einen Stammvokal *o hin.

(214) Der vokalische Auslaut schwindet (EKÄ 168). Ein Vergleich mit anderen ostseefinnischen Entsprechungen ergibt, dass für die zweite Silbe * $o$ ansetzbar ist (vgl. SSA (III: 315$)$ ).

(216) $-u$ ist wohl eine Ableitung. Als ursprünglicher Vokal ist in dieser Position * $a$ anzusetzen, vgl. fi. toukka 'Larve, Raupe; Wurm' wot. tõukka id. kar. toukka id. (SSA III: 315).

\section{GEGENPROBE}

Als Gegenprobe findet sich für diesen Lautwandel anhand von SSA und EES im Estnischen lediglich ein Beleg. Im Korpus sind keine weiteren Wörter in diesem Kontext relevant.

1 est. dial. (Kuusalu) koukima 'etw. von irgendwo/aus etw. herausziehen, hervorziehen; hervorstochern', kouk, gen. kougu 'Kartoffelhacke'

fi. koukku 'Haken, Angelhaken'

SSA (I: 414), EES (180f.)

1: Laut SSA (I: 414) stammt der estnische Beleg aus dem Dialekt Kuusalu und sei eine finnische Entlehnung.

Da nur ein Beleg für eine Gegenprobe angeführt werden kann und hierbei von einer dialektalen Vertretung auszugehen ist, scheint die Entwicklung der ersten Silbe bei diesem Lautwandel im Estnischen regelmäßig zu sein. Dies wird auch durch die Angaben aus der einschlägigen Literatur bestätigt. WIIK (1986: 43) erwägt eine regelmäßige Entwicklung * ou > õu im Estnischen. Auch KETTUNEN (EKÄ 141) stellt fest, dass die Entsprechung von *ou im Estnischen wohl immer õu sei. Holst (2001: 79f.) ist der Auffassung, dass dieser Diphthong seinem Labialitätsgesetz unterliege und daher den Lautwandel regelhaftig vollzieht. Dass sein Gesetz nicht gilt, konnte anhand der Entwicklung von $* o$ bereits festgehalten werden. 


\subsection{Wotisch}

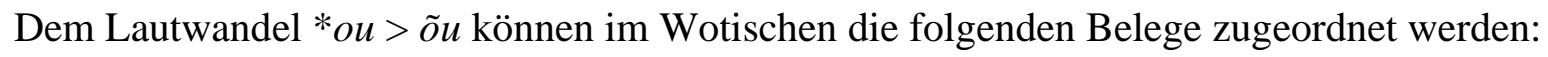
mit zweiter Silbe, die auf $* a$ zurückgeht:

(179) wot. jõutaa 'können, vermögen; Zeit haben',

(192) wot. lõunad 'Süd, Mittag, Mittagessen',

(204) wot. põuta 'trockenes, sonniges Wetter; Sonnenschein; Dürre',

(212) wot. sõutaa 'rudern',

(215) wot. tõukata 'stoßen, schieben',

(216) wot. tõukka 'Larve, Wurm';

mit zweiter Silbe, die auf *u zurückgeht:

(181) wot. jõukku 'Büschel, Menge, Gruppe, Verwandtschaft',

(182) wot. jõulu 'Weihnachten';

mit zweiter Silbe, die auf $* o$ zurückgeht:

(206) wot. rõukko '(auf Reitern, Reutern aufgeschichteter) Klee- oder Kornschober',

(214) wot. tõuko 'Sommersaat, -korn';

mit zweiter Silbe, die auf *e zurückgeht:

(178) wot. jõvi 'Pferdehaar, Rosshaar'.

\section{ANMERKUNGEN}

(178) Nach einem Diphthong mit $u$ als zweitem Element schwindet $h$, darauf folgt * $u>v$ (VKÄ 85). KetTunEn (ebd. 141) zufolge gab es gelegentlich Vermischungen mit den Formen der $i$ Stämme, sodass als Stammvokal hier $-e$ ansetzbar ist (vgl. auch weitere osfi. Entsprechungen).

\section{GEGENPROBE}

Die Zusammenstellung von Belegen für eine Gegenprobe fällt im Wotischen bescheiden aus.

1 wot. koukku 'Schürhaken aus Holz'

fi. koukku 'Haken, Angelhaken'

Laut SSA (I: 414) in vielen osfi. Sprachen belegt, est. < fi.; osfi. (EES 180f.)

1: Ob es sich hierbei um eine Entlehnung handelt oder um einen Teil des ursprünglichen Wortschatzes, ist anhand der etymologischen Wörterbücher nicht genauer zu bestimmen. Im Ingrischen ist gleichwohl ein derartiges Wort aus den Dialekten Saarove und Metsäkylä - also in 
unmittelbarer Nähe zum wotischen Sprachgebiet - zu verzeichnen, vgl. ing. koukku 'Schürhaken, mit dem man Kohlen aus dem Ofen holt; schwere Arbeit' (IMS 199).

Bei weiteren Wörtern, die sich in VKS finden, handelt es sich zumeist um solche, die etymologisch nicht näher bestimmbar sind und wohl dialektale Varianten oder Entlehnungen sein können (vgl. auch VKÄ 136). Einige Wörter sind dialektale Varianten von Wörtern, die auch in diesem Korpus vorliegen, z.B. wot. (Luuditsa) toukku 'Larve, Wurm', das hier durch (216) vertreten ist. ${ }^{77}$

Betrachtet man nun die wotischen Belege samt Gegenprobe, so lässt sich auch hier von einem Lautwandel ausgehen, der wohl regelmäßig ist. Für den einzigen gesicherten Gegenbeleg konnte ein Lösungsansatz ermittelt werden. Die Annahmen lassen sich auch durch die Lautlehre stützen: Der Lautwandel sei KETTUNEN (VKÄ 136) zufolge regelmäßig und ou komme nur in späteren Entlehnungen vor.

\subsection{Livisch}

Das Livische stellt auch beim Lautwandel $* o u>\tilde{o u}$ mit nur fünf einschlägigen Belegen die kleinste Vertretung dar. Zu ihnen zählen:

mit zweiter Silbe, die auf * $a$ zurückgeht:

(192) liv. lōnag 'Süd, Mittag, Mittagessen'.

(204) liv. põda 'neblig, schwül'.

(212) liv. sõudõ 'rudern'.

(215) liv. tevk, tẹk 'stoßen, stampfen';

mit zweiter Silbe, die auf $* e$ zurückgeht:

(178) liv. jõ'ugi 'aus Rosshaar'.

\section{ANMERKUNGEN}

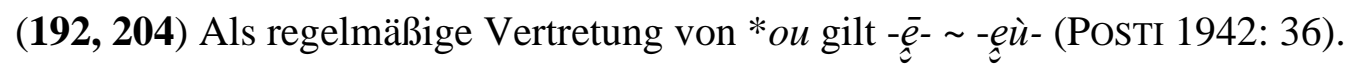

\footnotetext{
${ }^{77}$ Für den Dialekt Luuditsa (est. Luutsa) sind die gleichen Phoneme anzusetzen wie für weitere wotische Dialekte (vgl. VIITSO 1961: 144f.). Warum hier aber kein Lautwandel vorliegt, kann nicht ermittelt werden. Von Fremdeinfluss ist wohl bei derartigen Wörtern auszugehen, wenngleich es keine etymologisch gesicherten Nachweise gibt. Das Wotische, so auch dieser Dialekt, ist von anderen Sprachen deutlich beeinflusst (VIITSO 1961: 154).
} 
(215) Bei der Variante liv. tevk ist eine Entwicklung * $u>v$ beim zweiten Bestandteil des Diphthongs ansetzbar, vgl. est. tõugata 'stoßen, schieben' wot. tõukata id.

\section{GEGENPROBE}

Anhand von SSA und EES konnten für das Livische keine Belege für eine Gegenprobe zusammengestellt werden. Aus dem Korpus sind die folgenden zu nennen:

179 liv. joudõ 'können, vermögen'

fi. joutaa 'frei sein; Zeit haben'

190 ? liv. lōkandõks 'Eisloch'

fi. loukko 'Loch, Winkel'

(179) Posti (1942: 37) begründet die nicht stattgefundene Entwicklung mit einem möglichen Rückwirken der lettischen Wörter lett. jauda 'Vermögen, physische Kraft' und lett. jaudāt 'vermögen, können; verstehen, wissen' auf das livische Wort. Sie seien aus dem Ostseefinnischen entlehnt und für Posti (ebd.) ist dies „eine völlig befriedigende Erklärung“ für die erste Silbe. (190) Der Beleg wird nur als unsichere Entsprechung zu den anderen ostseefinnischen Sprachen gesehen. Die Vertretung von *ou in der ersten Silbe ist hier wohl -ō- -où- (PosTI 1942: 37). Aufgrund des ungesicherten Status ist dieser Beleg für eine Gegenprobe wenig nutzbringend.

Das Livische knüpft nach der Diskussion der Belege und der Gegenprobe an die bereits vorher diskutierten Sprachen an. Ein Lautwandel *ou > $\tilde{o u}$ ist auch in dieser Sprache wohl als regel-

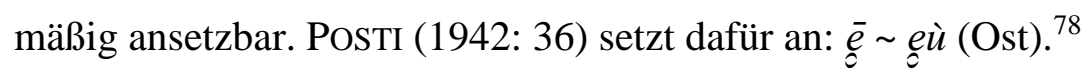

\subsection{Südestnisch}

Im Südestnischen sind 13 Belege für den Lautwandel *ou > $\tilde{u} u$ einschlägig. Sie verteilen sich, wie nachfolgend angeführt:

mit zweiter Silbe, die auf $* a$ zurückgeht:

(179) estS. .joudma, II. Infinitiv (.)joudag; .jõudma, II. Infinitiv (.)jõudaq 'können, vermögen; Zeit haben',

(189) estS. lõug, gen. lõvva 'Kinn',

\footnotetext{
${ }^{78} \mathrm{Zu}$ Ausnahmen, in denen der Diphthong keine Vertretung mit $\tilde{o}$ als Bestandteil hat, vgl. PosTI (1942: 37).
} 
(192) estS. lõunõq, gen. .lõunõ; lõunaq, gen. .lõuna; lõuna|k, gen. -gu 'Süd, Mittag, Mittagessen',

(198) estS. nõudma, II. Infinitiv (.)nõudaq 'verlangen, fordern',

(204) estS. põud, gen. põvva 'Dürre';

mit zweiter Silbe, die auf $* o$ zurückgeht:

(181) estS. jõuk, gen. jõugu 'Bande, Gruppe',

(190) estS. lõugõq, gen. .lõukõ 'gemauerte Seite des Aschenloches vor der Ofenmündung (als Sitz dienend), Ecke, Krümmung, Meerbusen',

(206) estS. rõuk, gen. rõugu '(auf Reitern, Reutern aufgeschichteter) Klee- oder Kornschober',

(212) estS. .sõudma, II. Infinitiv (.)sõudaq 'rudern',

(214) estS. tõug, gen. tõvvu 'Sommersaat, -korn';

mit zweiter Silbe, die auf *u zurückgeht:

(182) estS. joul, gen. joulu; jõul, gen. jõulu 'Weihnachten';

mit zweiter Silbe, die auf *e zurückgeht:

(178) estS. jõhv', gen. jõhvi; jõuh, gen. jõuhõ 'Pferdehaar, Rosshaar';

mit zweiter Silbe, deren Hintervokal nicht näher bestimmbar ist:

(186) estS. kõvve 'Donner'.

\section{ANMERKUNGEN}

(178) In einer der Varianten liegen Metathese des intervokalischen $h$ und das Verhältnis $u \sim v$ vor.

$(\mathbf{1 7 9}, \mathbf{1 8 2})$ Es existieren je Beleg zwei Varianten, wobei nicht eindeutig hervorgeht, welche die gebräuchlichere ist. An den für den Lautwandel einschlägigen Formen ist nichts Ungewöhnliches.

(186) Vgl. Kapitel 5.2.2.1.1 Estnisch. Ein Hintervokal kann wohl auch im Südestnischen als ursprünglicher Stammvokal angesetzt werden. Abermals liegt $u \sim v$ vor, $v$ wurde dazu geminiert. 


\section{GEGENPROBE}

Ein Beleg konnte anhand von SSA und EES für das Südestnische für einen Gegenbeweis ermittelt werden:

1 estS. kougi|tsõma, -tsaq -daq 'etw. von irgendwo/aus etw. herausziehen, hervorziehen; hervorstochern'

fi. koukku 'Haken, Angelhaken'

SSA (I: 414), EES (180f.)

1: Für die est. Entsprechung wurde eine finnische Entlehnung angesetzt. Ob bei der südestnischen Form von Einfluss aus dem Standardestnischen auszugehen ist, ist etymologisch nicht bestätigt, jedoch im Bereich des Möglichen.

$\mathrm{Zu}$ ergänzende Belege aus dem Korpus sind:

179 estS. .joudma, II. Infinitiv (.)joudag 'können, vermögen; Zeit haben'

fi. joutaa 'frei sein; Zeit haben'

182 estS. joul, gen. joulu 'Weihnachten'

fi. joulu 'Weihnachten'

215 estS. .toukama, II. Infinitiv tougadaq; .tuukama, II. Infinitiv tuugadaq [...] 'stoßen, schieben'

fi. toukata 'reißen, zupfen; schubsen [...]'

$(\mathbf{1 7 9}, \mathbf{1 8 2})$ Aus der Betrachtung der einschlägigen Belege (s.o.) ist bereits hervorgegangen, dass auch Varianten vorliegen, in denen der Lautwandel vollzogen wird. Die hier genannten Formen können daher wohl als sekundär bewertet werden.

(215) Dem Beleg wird ein deskriptiver Wortcharakter zugeschrieben, der Grund für die Abweichung sein kann.

Vier Belege konnten für die Gegenprobe angesetzt werden. Lediglich bei einem von ihnen wurde keine Erklärung für die Wortentwicklung gefunden, sodass auf dieser Grundlage und vor dem Hintergrund der anderen Sprachen wohl auch für das Südestnische ein regelmäßiger Lautwandel *ou > $\tilde{o u}$ angesetzt werden kann. 
Der Lautwandel *eu > $\tilde{o} u$ ist nur in sehr eingeschränkter Anzahl in diesem Korpus vertreten. In allen Sprachen durchlaufen ihn jeweils genau zwei Belege, die für die jeweiligen Einzelsprachen in den nächsten Kapiteln genauer betrachtet werden sollen.

\subsection{Estnisch}

Die für die Entwicklung *eu > $\tilde{u}$ im Estnischen einschlägigen Belege sind:

(189) est. $l \tilde{o} u \mid g$, gen. - $a$ 'Kinn',

(197) est. nõu 'Rat, Ratschlag'.

\section{ANMERKUNGEN}

(197) Der vokalische Auslaut wird apokopiert (EKÄ 168), woraufhin nach dem Labialvokal der Schwund von $v$ eintritt (ebd. 116). Der alte Stammvokal lautete *o, vgl fi. neuvo 'Rat; Art und Weise; Ratschlagung [...]'.

\section{GEGENPROBE}

Für eine Gegenprobe konnten weder anhand des Korpus noch der etymologischen Wörterbücher Belege zusammengestellt werden, sodass davon auszugehen ist, dass die Entwicklung *eu $>\tilde{o} u$ trotz ihrer sehr geringen Anzahl an Belegen regelmäßig ist. Das Ergebnis stimmt mit den Angaben überein, die in der Fachliteratur gemacht werden: VIITSO (2003: 22) führt beispielsweise an, dass der Diphthong eu im Estnischen nur in Entlehnungen auftrete. HoLST (2001: 79) bezeichnet den Lautwandel $* e u>\tilde{o u}$ für das Estnische ebenfalls als regelmäßig, WIIK (1986) geht in seinen Ausführungen nicht darauf ein. In früheren Arbeiten wie denen von KETTUNEN (EKÄ 141) wird auf diesen Lautwandel ebenso wenig Bezug genommen, da man davon ausging, dass $\tilde{o} u$ alt sei.

\subsection{Wotisch}

Im Wotischen sind für den Lautwandel *eu > õu die nachfolgenden Belege einschlägig: (189) wot. lõuka 'Kinn', (197) wot. nõvvo 'Rat, Ratschlag'. 


\section{ANMERKUNGEN}

(197) Eine Entwicklung -evav- > -evv- (VKÄ: 137) tritt ein.

\section{GEGENPROBE}

Auf Grundlage der etymologischen Wörterbücher konnten zwei Belege für die Gegenprobe zusammengestellt werden.

1 wot. peiko, (Must) peukolo 'Daumen'

fi. peukalo 'Daumen'

SSA (II: 346), EES (406)

2 wot. (Tsv) seukko 'Cousin(e)'

fi. $\operatorname{seukku}$ 'Cousin(e)'

? $<$ fi. (SSA III: 171)

1: Zwei unterschiedliche Formen können identifiziert werden, wobei sich jene mit $-e i$ - in der ersten Silbe auf eine der beiden von SSA (II: 346) angesetzten Urformen zurückgeht, jene mit -eu- auf die andere, vgl. peikalV *peukalV. Warum der Lautwandel nicht eintritt, ist nicht erklärbar. $^{79}$

2: Dem Beleg wird zugeschrieben, nach TSVETKOV notiert sowie eine mögliche Entlehnung aus dem Finnischen zu sein, sodass er für die Gegenprobe nicht nutzbringend ist.

In einzelnen Dialekten finden sich ferner Beispiele, in denen zwar das *eu bewahrt ist, da sie etymologisch jedoch nicht sicher belegt sind, ist uneindeutig, welche Entwicklung sie durchlaufen haben, vgl. dazu z.B. wot. keula 'Bug (des Schiffes oder Bootes)', das in SSA (I: 351f.) nicht mit fi. keula 'Bug; Vordach; Mützenschirm [...]' zusammengestellt wird. Wirft man noch einen Blick auf die Lautlehre, so ist festzuhalten, dass man von einem alten $\tilde{o} u$ ausging und keinen Bezug auf $* e u$ genommen hat (VKÄ 136). Eine Entwicklung $* e u>\tilde{o u}$ wird dadurch nach heutigem Stand impliziert.

\footnotetext{
${ }^{79}$ Die Entsprechung est. pöial 'Daumen' hat eine eigenständige Entwicklung durchlaufen und ist für die Untersuchung im Estnischen nicht einschlägig.
} 


\subsection{Livisch}

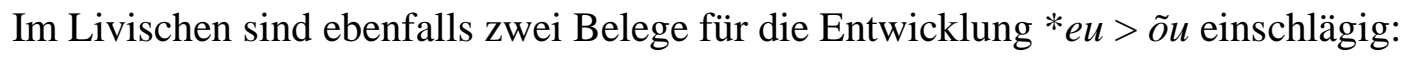

(189) liv. lõga 'Kinn',

(197) liv. nõ'v 'Rat, Ratschlag'.

\section{ANMERKUNGEN}

(189) $\tilde{\tilde{o}}$ ist die regelmäßige Vertretung von õu (vgl. PosTi 1942: 37)

(197) Der vokalische Auslaut -o ist im Livischen immer geschwunden (POSTI 1942: 62).

\section{GEGENPROBE}

Für das Livische konnten weder anhand der etymologischen Wörterbücher noch unter Zuhilfenahme von LELS Belege ermittelt werden, in denen *eu bewahrt wurde.

Obschon nur zwei einschlägige Belege vorliegen, ist der Lautwandel im Livischen mangels Gegenbeweise wohl als regelmäßig zu bewerten. Dies deckt sich auch mit PosTIS (1942: 37f.) Ausführungen zum Livischen, in denen er von einem alten $\tilde{o} u$ ausgeht und keine Entwicklung mit $* e u$ diskutiert.

\subsection{Südestnisch}

Im Südestnischen sind, ebenso wie in den anderen Sprachen, die nachfolgenden beiden Belege relevant:

(189) estS. lõug, gen. lõvva 'Kinn',

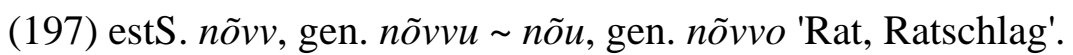

\section{ANMERKUNGEN}

(189, 197) Das Verhältnis $u \sim v$ liegt vor, $v$ wird geminiert.

\section{GEGENPROBE}

Anhand des Korpus und den etymologischen Wörterbüchern konnten keine Belege für eine Gegenprobe ermittelt werden. Ebenso wenig liegen laut EVS Wörter vor, in denen das *eu in der ersten Silbe vorliegt. Es kann also von der Gültigkeit des Lautwandels ausgegangen werden. 


\subsubsection{Diphthonge mit $i$ als zweitem Bestandteil}

Die Anzahl der einschlägigen Diphthong-Belege mit $i$ als zweitem Bestandteil ist recht überschaubar. Sie verteilen sich v.a. auf *ei und *oi (*ei: Estnisch vier Belege, Wotisch drei, Livisch ohne, Südestnisch zwei; *oi: Estnisch 12 Belege, Wotisch elf, Livisch sechs, Südestnisch zehn).

\subsubsection{1 *ei> $\tilde{o} i$}

Der Lautwandel $* e i>\tilde{o} i$ ist nicht sehr stark vertreten, wie die nachfolgende Übersicht darlegt:

$\begin{array}{ccccc}\text { Vokal der zweiten Silbe } & \text { Estnisch } & \text { Wotisch } & \text { Livisch }^{80} & \text { Südestnisch } \\ a & 2 & 2 & 0 & 2 \\ o & 1 & 1 & 0 & 1 \\ e & 1 & 0 & 0 & 0\end{array}$

Wenngleich die Beleganzahl insgesamt gering ist, deutet die Verteilung darauf hin, dass der Lautwandel wohl dann auftritt, wenn die zweite Silbe hintervokalisch ist.

\subsection{Estnisch}

Die vier einschlägigen Belege verteilen sich im Estnischen folgendermaßen:

mit zweiter Silbe, die auf *a zurückgeht:

(184) est. kõikuda 'sich bewegen, schwanken, schaukeln',

(187) est. lõigata 'schneiden';

mit zweiter Silbe, die auf $*$ o zurückgeht:

(177) est. hõim, gen. - $u$ 'Familie, Sippe';

mit zweiter Silbe, die auf $* e$ zurückgeht:

(200) est. õis, gen. ôie õile, gen. ôilme 'Blüte'.

\section{ANMERKUNGEN}

(177) Ein Lautwandel $*_{o}>u$ tritt in nichterster Silbe ein (EK ̈̈ 155).

\footnotetext{
${ }^{80}$ POSTI (1942: 35) setzt an, dass * ei durch $\bar{e} \sim$ eì vertreten sei und nennt keine Beispiele, in denen ein Lautwandel zu $\tilde{o} i$ als Resultat auftritt. Über $\tilde{o} i$, welches er als ursprünglich annimmt, sagt er, dass es keine Beispiele mit diesem Diphthong gebe, deren Entsprechung im Finnischen $e i$ und im Estnischen ebenso õ $i$ sei (ebd. 35). Diese Aussage stützt, dass es im Livischen keinen Lautwandel $* e i>\tilde{o} i$ gegeben hat.
} 

) $-u$ ist eine Ableitung, als Stamm wird urfi. *keikka (LÄGLOS II: 73) angesetzt.

(200) Aufgrund der hohen Vielfalt an Varianten ${ }^{81}$ ist von einem stark onomatopoetisch beeinflussten Wort auszugehen. Daher ist es bei der Identifikation von regelmäßigen Entwicklungen nicht von Nutzen. Der Status als Onomatopoetikum muss die Begründung für die hiesige Entwicklung *ei $>\tilde{o} i$ sein.

\section{GEGENPROBE}

Für eine Gegenprobe zur These, dass der Lautwandel bei hintervokalischen Folgesilben eintritt, sind die nachfolgenden Belege anzuführen:

$1 \quad$ est. hein, gen. heina 'Heu'

fi. heinä 'Gras, Heu'

< balt. (SSA I: 150f., EES 72f.)

2 est. heita 'werfen, schleudern'

fi. heittää '(ver)werfen, aufgeben'

*heittä- (LÄGLOS I: 91)

< germ. (SSA I: 151), < ? urgerm. (EES 73), germ. Lehnwort (LÄGLOS I: 91)

3 est. leib, gen. leiva 'Brot'

fi. leipä 'Brot'

*leipä (LÄGLOS II: 190)

< germ. (SSA II: 62, EES 234f.), germ. Lehnwort (LÄGLOS II: 190)

$4 \quad$ est. lein, gen. leina 'Trauer' (gen. laut SSA auch - $\ddot{a},-o,-u$ )

fi. leina 'schlechter Getreidewuchs; schwach, elend'

? < balt. (SSA II: 60, EES 235)

5 est. neid, gen. neiu 'junge, unverheiratete Frau; Mädchen, Tochter der Tochter'

fi. neiti 'Fräulein, Jungfrau', neito 'junge Dame; Jungfer; Mädchen'

SSA (II: 211f.), EES (309f.)

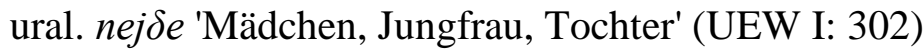

\footnotetext{
${ }^{81}$ Ferner können genannt werden: öil, oil(e), oilme, õilme(s), õiles (VMS II: 732); häälme, (h)ödremed (Plural), öormed (Plural) [...] (SSA I: 150) (vgl. auch das Korpus oben). Es ist nicht auszuschließen, dass auch noch weitere Varianten existieren.
} 
est. peig, gen. peiu 'Bräutigam' liv. (Sal) peikil- in peikilmies id.

? fi. peikko 'böser Geist, Unhold, Kobold; widerspenstiges, bösartiges Wesen (Mensch oder Tier)'

SSA (II 330); ? < urgerm. oder ? < balt. (EES 359f.); LÄGLOS (III: 43f.) behandelt est. und liv. nicht

est. peita 'verstecken'

fi. peittää 'verstecken'

urfi. *peittää (LÄGLOS III: 44)

SSA (II: 331), ? < urgerm. (EES 360), germ. Lehnwort (LÄGLOS III: 44)

8 est. sein, gen. seina 'Wand'

fi. seinä 'Wand'

< balt. (SSA III: 164, EES 465)

9 est. teivas, gen. teiba 'Pfahl, Stange, Stab'

fi. seiväs 'Pfahl, Stange, Stab'

< balt. (SSA III: 165, EES 522)

10 est. veider, gen. veidra 'sonderbar, seltsam'

fi. veiterä 'tüchtig, geschickt, gewandt, flink'

SSA (III: 422), EES (597)

11 est. vesta 'schnitzen'

fi. veistää 'schnitzen'

SSA (III: 422f.), EES (599f.)

ural. wä̈ćs 'Messer; schneiden' (UEW I: 565)

1-3, 7-9, 11: Der Abbau der Vokalharmonie führte zur Veränderung der zweiten Silbe.

4: Da es sich womöglich um eine baltische Entlehnung handelt, kann eine hintervokalische zweite Silbe erwogen werden. Die Verhältnisse sind aber nicht eindeutig genug, dies gesichert zu ermitteln oder zu bestimmen, warum der Lautwandel in der ersten Silbe nicht eintritt.

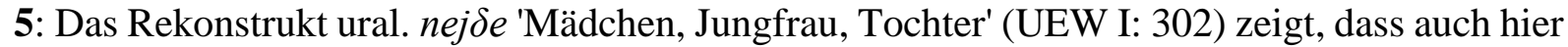
der Stamm ursprünglich vordervokalisch gewesen sein muss. Daher ist est. $-u$ wohl eine Ableitung. Die Annahme lässt sich stützen durch die finnische Entsprechung, für die in SSA (II: 211f.) ebenso eine Ableitung angesetzt wird

6: Die Verbindung zur fi. Entsprechung ist nicht gesichert, die Ursache für das Bewahren von *ei konnte nicht ermittelt werden. 
10: Ein alter Stammvokal konnte nicht ermittelt werden. Der Wortcharakter ist onomatopoetisch-deskriptiv, was wohl der Grund für die Abweichung ist.

Aus dem Korpus ist noch ein weiterer Beleg zu ergänzen:

209 est. seista 'stehen'

fi. seisoa 'stehen'

(209) Da keine weiterführenden Angaben vorliegen, konnte nicht ermittelt werden, warum ein Lautwandel hier nicht eintritt.

Zwölf Belege wurden anhand der Gegenprobe betrachtet, zehn von ihnen ließen sich aufklären.

Da die Abweichung sehr gering ist, kann trotz der bescheidenen Beleganzahl für einen Lautwandel $* e i>\tilde{o} i$ angesetzt werden, dass dieser im Estnischen normalerweise bei einem Hintervokal in der zweiten Silbe eintritt. Die Erkenntnis knüpft an die These von Holst (2001: 79) an, der davon ausgeht, dass sich $i$ in zweiter Position des Diphthongs neutral verhalte und die Lautwandel so vollzogen werden, wie bei den Kurzvokalen (also $* e>\tilde{o}$ bei einem Hintervokal in der zweiten Silbe). WIIK (1986: 43f.) definiert die Lautwandel der Diphthonge für das Estnische kaum. Auf $* e i$ geht er, wie auch KeTtUNEN (EKÄ), nicht ein.

\subsection{Wotisch}

Die nachfolgenden Belege sind im Wotischen für den Lautwandel einschlägig:

mit zweiter Silbe, die auf *a zurückgeht:

(187) wot. lõikata 'schneiden',

(209) wot. sõisa 'stehen';

mit zweiter Silbe, die auf *o zurückgeht:

(177) wot. öimo 'Familie, Sippe'.

\section{ANMERKUNGEN}

$\mathrm{Zu}$ den Belegen sind keine Ergänzungen anzuführen.

\section{GEGENPROBE}

Für die Gegenprobe konnten die nachfolgenden Belege zusammengestellt werden: 
wot. (Ahlqvist) eikko 'mager, dünn', (Kukk) heikko 'schwach; dünn; schlecht'

fi. heikko 'schwach; dünn; schlecht'

SSA (I: 150)

wot. (Kukk.; Volksdichtung) neijjod (Pl.), neijukkaina, neito, neite 'junge, unverheiratete Frau; Mädchen, Tochter der Tochter'

fi. neiti 'Fräulein, Jungfrau', neito "

SSA (II: 211f.), EES (309f.)

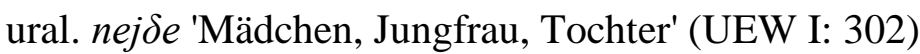

1: Für die Variante aus dem Dialekt Kukkuzi ist festzuhalten, dass $\tilde{o}$ in der ersten Silbe nicht auftritt. Die zweite Variante liegt lediglich in der Aufzeichnung nach Ahlqvist vor. Ihre Bewertung ist fraglich.

22: Dieser Beleg wird ebenfalls dem Dialekt Kukkuzi zugeordnet und gilt ferner als Form aus der Volksdichtung.

Aus dem Korpus ist zu ergänzen:

184 wot. keikab 'schaukeln-3SGPRÄs'

fi. keikkua 'schaukeln', keikka 'hoch und nach hinten gekrümmt; Kufe einer Wiege

$[\ldots]^{\prime}$

urfi. *keikka (LÄGLOS II: 73)

(184) Der Wortstamm wird in den etymologischen Wörterbüchern als deskriptiv bezeichnet, was Ursache für die Abweichung sein muss.

Betrachtet man nun die Belege, die im Wotischen im Korpus und in der Gegenprobe vorliegen, so lässt sich festhalten, dass auch hier der Lautwandel $* e i>\tilde{o} i$ gültig scheint, wenn in der Folgesilbe ein Hintervokal auftritt. KETTUNEN trifft über eine Entwicklung keine Aussage. Die Belege, die er für wot. *ei notiert, sind allesamt in der Folgesilbe vordervokalisch (VKÄ 134). 


\subsection{Livisch}

Der Lautwandel *ei > $\tilde{o} i$ liegt im Livischen nicht vor, daher wird an dieser Stelle auf eine Zusammenstellung von Belegen verzichtet, in deren erster Silbe *ei bewahrt ist. Auf eine Abweichung aus dem Korpus soll dennoch eingegangen werden:

(177) liv. aim 'Familie, Sippe'

PosTi (1942: 35) setzt für diesen Beleg sowie einige weitere vom „livischen Standpunkt aus [...] eine späturfinnische Ausgangsform mit ai voraus“, vgl. z.B. liv. àina 'Heu' fi. heinä id.

est. hein, gen. heina id. Eine Begründung für dieses Verhältnis wird von ihm nicht angeführt. Baltischen Einfluss auf die Lautung dieses Wortes anzusetzen, scheint möglich, vgl. lett. saime 'Gutsleute; Familie (im weitesten Sinne)' (SSA I: 150).

\subsection{Südestnisch}

Die nachfolgenden Belege sind im Südestnischen einschlägig:

mit zweiter Silbe, die auf *a zurückgeht:

(184) estS. .kõikma, II. Infinitiv .kõikuq 'sich bewegen, schwanken, schaukeln',

(187) estS. .lõikama, II. Infinitiv lõigadaq 'schneiden';

mit zweiter Silbe, die auf *o zurückgeht:

(177) estS. hõim, gen. hõimo 'Familie, Sippe'.

\section{ANMERKUNGEN}

(184) Als Stamm wird urfi. *keikka (LÄGLOS II: 73) angesetzt, folglich ist bei $-u$ von einer Ableitung auszugehen.

\section{ABWEICHUNGEN IN DER ERSTEN SILBE}

(200) estS. häilmü, häiermü 'Blühen, Blüte'; häidseq, gen. .häits(m)e; häierm; häielm; häelm 'Blüte'

(209) estS. .saisma, II. Infinitiv (.)saistaq 'stehen'

(200) Bereits im Vorfeld wurde der onomatopoetische Charakter dieses Belegs besprochen. Für das Südestnische konnten ebenfalls verschiedene Varianten ermittelt werden, allerdings keine, in der der Laut $\tilde{o}$ auftritt. 
(209) Ein Stamm fiugr. sayća 'stehen' wird angesetzt (UEW I: 431f.). Zwar behandelt man das Südestnische dort nicht, für fi. und est. wird gleichwohl ein sekundärer Wandel $* a j>e i$ angesetzt, der für die Unterschiede estS. ai und est. $\tilde{o} i(<* e i)$ verantwortlich sein kann.

\section{GEGENPROBE}

Für eine Gegenprobe konnten anhand von SSA und EES sowie des Korpus keine südestnischen Belege ermittelt werden. Vereinzelte Wörter, die in EVS anzutreffen sind, werden etymologisch nicht behandelt und können somit nicht in die Bewertung einfließen, z.B. estS. leik, gen. leigu 'Trichter'.

Der Lautwandel $* e i>\tilde{o} i$ bei einem Hintervokal in der Folgesilbe scheint für das Südestnische auch hinsichtlich der Gegenprobe trotz geringer Beleganzahl gültig.

\subsubsection{2 $* o i>\tilde{o i}$}

Die Vertretung der einschlägigen Belege des Lautwandels * oi $>\tilde{o} i$ ist die insgesamt zweitstärkste in der Kategorie der Diphthonge. Im Estnischen unterliegen dem Lautwandel $* o i>\tilde{o} i$ insgesamt 12 Belege, im Wotischen elf, im Südestnischen zehn sowie im Livischen sechs. Das nachfolgende Diagramm veranschaulicht die Verteilung der Belege anhand ihrer Folgesilbe. Anhand des Diagrammes zeigt sich, dass zwar die hintervokalischen Folgesilben insgesamt dominieren, ein Lautwandel daran jedoch nur bedingt festzumachen ist. Dies deutet darauf hin, dass der Lautwandel wohl unabhängig von der zweiten Silbe eingetreten sein kann.

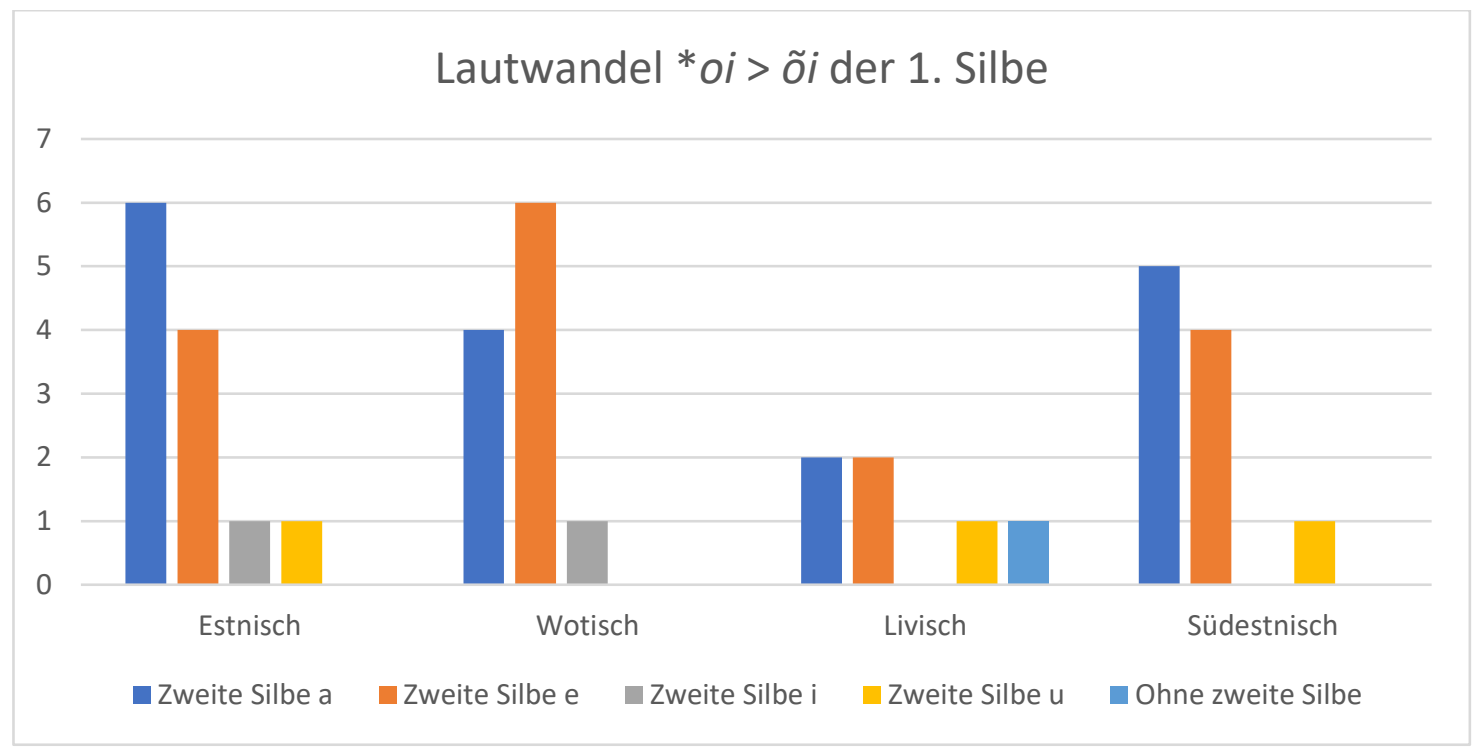




\subsection{Estnisch}

Die für das Estnische einschlägigen Belege verteilen sich, wie nachfolgend ausgeführt: ${ }^{82}$ mit zweiter Silbe, die auf $* a$ zurückgeht:

(193) est. mõis, gen. - $a$ 'Gut, Landgut', (205) est. rõivas 'Kleid, Kleidung', (196) est. nõi|d, gen. - $a$ 'Zauberer, Hexe', (210) est. sõita 'fahren', (202) est. põigata 'abbiegen', (221) est. võida 'können';

mit zweiter Silbe, die auf *e zurückgeht:

(188) est. lõim, gen. - $e$ 'Weberkette', (208) est. sõim, gen. - $e$ 'Krippe', (199) est. öige 'recht, richtig', (219) est. või 'Butter';

mit zweiter Silbe, die auf $* i$ zurückgeht:

(203) est. põimida 'flechten, winden';

mit zweiter Silbe, die auf * $u$ zurückgeht:

(185) estS. kõiv, gen. - $u$ 'Birke'.

Da sich die Belege hier sehr unterschiedlich verteilen, ist davon auszugehen, dass ein Lautwandel $* o i>\tilde{o} i$ unabhängig von der Folgesilbe eintreten kann. Auffällig ist außerdem, dass ein Lautwandel nicht vorkommt, wenn der zweite Diphthongbestandteil zu $e$ wird. Wörter, wie z.B. est. koer, gen. - $a$ 'Hund', sind für die Entwicklung also nicht empfänglich. Zu den Bedingungen der Entwicklung *Vi> Ve s. KETTUNEN (EKÄ 140).

\section{ANMERKUNGEN}

(185) Angesetzt wurde hier die südestnische Entsprechung als reguläre Vertretung des Belegs. ${ }^{83}$

(219) Als Urstamm wird fiugr. woje 'Fett, Fettstoff' (UEW I: 578) rekonstruiert, sodass *e als Vokal der zweiten Silbe in einer früheren Form vorgelegen haben muss.

\footnotetext{
${ }^{82}$ WIIK (1986: 43f.) führt außerdem für die Imperfektformen der Verben est. jooma 'trinken', est. looma '(er)schaffen, bilden; [...]' und est. tooma 'bringen, holen' morphophonologische Entwicklungen an, die durch den Eintritt des Imperfektmarkers ausgelöst werden und für den Lautwandel * $o i>\tilde{o} i$ regelmäßig seien: joon 'trinken-1SGPRÄS' : jõin id.-1SGIMP; toon 'bringen, holen-1SGPRÄs': tõin id.-1SGIMP. Ferner gehört zu dieser Gruppierung est. loon '(er)schaffen [...]-1SGPRÄs' : lõin id.-1SGIMP. Das Verb est. sööma 'essen' wird von WIIK (ebd. 43) nicht behandelt, wohl weil aufgrund einer anderen Vertretung der ersten Silbe eine andere Entwicklung stattfindet. Die Imperfektform verhält sich jedoch wie die der bereits genannten Verben: sö̈on 'essen-1SGPRÄS' : sõin id.-1SGIMP. Womöglich handelt es sich hierbei um eine analoge Entwicklung.

${ }^{83} \mathrm{Vgl}$. auch estN. kask 'Birke.', das etymologisch zu fi. kaski 'Schwende; junger Laubwald; junge Birke' gestellt wird und nicht mit estS. kõiv in Verbindung zu bringen ist (SSA I: 323, 386).
} 


\section{ABWEICHUNGEN IN DER ERSTEN SILBE}

(213) est. teine 'andere/r/s, zweite/r/s'

In den etymologischen Wörterbüchern wird eine Ableitung vom Pronomen fi. tuo 'jener' bzw. est. too id. angesetzt (SSA III: 304; EES 522). In SSA (III: 327f.) geht man bei fi. tuo von einem osfi. *tō mit wechselnder Vertretung aus, in EES (538) wird eine uralische Form erwogen. Diese Annahmen geben jedoch keine Auskunft über die abweichende Entwicklung der ersten Silbe. VIITSO (1978b: 94) schlägt für diesen Beleg eine Folge von Lautwandeln vor: $*_{o}>\tilde{o}>$ $e$. Da keine weiteren Abweichungen dieser Art für einen Vergleich herangezogen werden können, ist diese Vermutung nicht gesichert. Der Lautwandel scheint unmotiviert.

\section{GEGENPROBE}

Für eine Gegenprobe konnten zusammengestellt werden:

1 est. hoida 'festhalten; beschützen; bewahren; bewachen'

fi. hoitaa 'für jemanden sorgen, jemanden pflegen'

SSA (I: 169), EES (77f.)

2 est. koi, kojo 'Motte'

fi. $k o i$ 'Motte'

SSA (I: 384), EES (171)

fiugr. koje koja (UEW I: 167)

3 est. koi-valge 'Abendrot' (EES auch: est. koit, gen. koidu 'Morgenrot')

fi. koi 'Morgendämmerung; Osten'

SSA (I: 383), EES (171)

fiugr. koje 'Morgenröte' (UEW I: 167)

4 est. koib, koiv, gen. koiva '(Hinter)bein, Oberschenkel, Keule, Schenkel; Rand des Fells mit Beinteilen, Überbleibsel des Leders'

fi. koipi '(Hinter)bein, Oberschenkel, Keule'

SSA (I: 384), EES (171)

5 est. loib, gen. loiva 'Flosse, Pfote' liv. lūoiba 'Bundschuh aus Robbenflossen; Robbenflosse'

EES (246), in SSA nicht behandelt

6 est. loid, gen. loiu 'matt, träge, flau', SSA: loidata 'faulenzen'

fi. dial. loitia '(müßig) liegen, faulenzen'

SSA (II: 88), EES (246) 
7 est. loik, gen. loigu 'Lache, Pfütze' liv. luoik 'Tal; flaches Gebiet, Sumpf'

? fi. loikkua 'überschwappen, vergossen werden'

EES (246); laut SSA (II: 87) ist die Verbindung von fi. zu est. und liv. unsicher

8 est. loim, gen. loimu '(unvollständig zugewachsene) Baumwunde, Höhlung im Baum'

fi. loima '(unvollständig zugewachsene) Baumwunde, Höhlung im Baum'

SSA (II: 87), EES (246)

9 est. oiata 'ächzen, stöhnen'

wot. oigata, oikkia 'ächzen, stöhnen; klagen'

EES (335)

10 est. oim, gen. oimu 'Schläfe, Verstand'

fi. oimo in Wörtern oimolla(an), oimossa 'bei Sinnen, gesund'

SSA (II: 261), EES (335)

11 est. oinas, gen. oina 'Schafbock, Widder, Hammel'

fi. oinas 'Hammel, Schafbock'

$<$ balt. (SSA II: 261, EES 335)

12 est. (dial.) poiss 'weg'

fi. pois 'weg'

SSA (II: 385f.); in EES nicht behandelt

13 est. roiduda 'ermatten, ermüden'

fi. dial. roitua 'ermüden, schwach werden'

EES (433), in SSA nicht behandelt

14 est. roisk, gen. roisu 'Moder, Fäulnis; Gesindel'

fi. dial. roiska 'alte, elende Maschine' (SSA: 'Rute, Peitsche, Reisig')

EES (434), in SSA (III: 88) nicht zusammengestellt

15 est. roid, gen. roiu 'grober Kies, Staub, Schmutz'

fi. roitto 'Abfall; Fetzen, Lumpen'

SSA (III: 88), in EES nicht behandelt

16 est. soikuda 'sich beruhigen' liv. suoīkõl 'leise, ruhig'

EES (478), in SSA nicht behandelt

17 est. toimi, gen. toime, toime panna 'bewerkstelligen, ausführen, verrichten'

fi. toimi 'Beschäftigung, Arbeit [...]'

Urstamm *toj(3)- (SSA III: 304), EES (535) 
1, 4, 5, 11: Der Lautwandel tritt nicht ein. Der Laut $a$ ist in diesen Belegen der Vokal der zweiten Silbe.

2: Warum der Lautwandel in diesem Beleg nicht eintritt, kann nicht geklärt werden.

3: Warum kein Lautwandel eintritt, kann nicht ermittelt werden. Bei der Variante est. koit, gen. koidu kann wohl von einer Ableitung ausgegangen werden.

6, 7, 9, 13-16: Aufgrund ihres onomatopoetischen oder deskriptiven Charakters liegt bei diesen Belegen wohl kein Lautwandel vor.

8: Im Vergleich zu weiteren osfi. Sprachen ist * $a$ als Vertretung der zweiten Silbe wohl ansetzbar, vgl. fi. loima '(unvollständig zugewachsene) Baumwunde, Höhlung im Baum', kar. loima id. (SSA II: 87).

10: Sofern der Beleg zu fi. oiva gestellt werden kann (vgl. SSA II: 261), ist von einem * $a$ in der zweiten Silbe auszugehen.

12: Für die est. Entsprechung wird eine dialektale Form angesetzt, die Entsprechungen in den anderen osfi. Sprachen ermöglichen keine eindeutige Bestimmung der Folgesilbe, vgl. fi. pois 'weg' (dial. auch poi(j)es, poikes [...]) oder wot. poizag, poizek, poiz(a) id. (SSA II: 386).

17: Die ursprüngliche Lautung der zweiten Silbe ist nicht zu ermitteln, in einer osfi. Stufe ist sie wohl als vordervokalisch ansetzbar.

Aus dem Korpus kommt hinzu:

220 est. voi (Wied.) '(Schmerz ausdrückend; Interjektion)'

fi. voi 'ach!, oh!; au! [...]'

(220) Die Abweichung kann über den Status als Interjektion erklärt werden.

Betrachtet man die Ergebnisse der Gegenprobe, so fällt auf, dass der Lautwandel * $o i>\tilde{o} i$ häufig nicht eintritt, wenn die Folgesilbe $a$ lautet. Die Annahme, der Lautwandel trete unabhängig vom Laut der zweiten Silbe ein, muss also um diese Erkenntnis erweitert werden. Die Entwicklung von *oi ist demzufolge ähnlich zweigeteilt wie die des *o und nicht lautgesetzlich.

Die Neutralität des Diphthongbestandteils $i$ nach HOLST (2001: 79) wurde bereits bei der vorherigen Entwicklung genannt. So scheint auch hier eine Entwicklung $*_{o}>\tilde{o}$ bei einem Vordervokal in der zweiten Silbe zu gelten. WIIK (1986: 43f.) bemerkt zu *oi bzw. *o im Allgemeinen, dass es mal erhalten bleibt, mal dem Lautwandel unterliegt. Lediglich dass das $i$ im Diphthong einen Lautwandel des ersten Bestandteils zu õ auslöst, wenn es der Imperfektmarker ist, hält er 
als Regel fest. KETTUNEN (EKÄ 139f.) schreibt ebenfalls lediglich, dass es auch im Falle von $o i \sim \tilde{o} i$ eine Zweigeteiltheit gibt, wie schon bei $* o$. All diese Aussagen stehen nicht im Widerspruch zu den Ergebnissen dieser Betrachtung, wenngleich sie nicht alle Punkte umfassen.

\subsection{Wotisch}

Im Wotischen verteilen sich die Belege des Lautwandels * $o i>\tilde{o} i$ wie folgt:

mit zweiter Silbe, die auf $* a$ zurückgeht:

(193) wot. mõiza 'Gut, Landgut',

(210) wot. sõittaa 'fahren; schelten',

(196) wot. nõita 'Zauberer, Hexe',

(221) wot. vęija/võitaa 'können';

mit zweiter Silbe, die auf *e zurückgeht:

(188) wot. lõim $\mid i$, gen. - $\tilde{o}$ 'Weberkette',

(213) wot. tõin, gen. tõizõ̃ 'andere/r/s,

(199) wot. õikõa 'recht, richtig, grade', zweite/r/s',

(202) wot. põitši '(postp.) seit, ab (zeitlich)',

(219) wot. või 'Butter';

(208) wot. sõim|i, gen. -õo '(Pferde)krippe',

mit zweiter Silbe, die auf $* i$ zurückgeht:

(203) wot. põimia 'sammeln, pflücken'.

\section{ANMERKUNGEN}

(219) Nach dem rekonstruierten Stamm fiugr. woje 'Fett, Fettstoff' (UEW I: 578) zu schließen, ist der Beleg jenen mit * $e$ in der Folgesilbe zuzuordnen.

Im Wotischen liegt ebenso ein gemischtes Verhältnis vor, sodass zunächst davon auszugehen ist, dass der Lautwandel auch in dieser Sprache unabhängig von der Folgesilbe eintritt.

\section{GEGENPROBE}

Im Wotischen sind die nachfolgenden Belege Bestandteil der Gegenprobe:

1 wot. koi 'Motte'

fi. $k o i$ 'Motte'

Keine Herkunftsangabe in SSA (I: 384); fiugr. (EES 171)

UEW (I: 167): koje koja (fiugr.)

2 wot. koinattẹb 'bellen, schimpfen'

fi. koinata 'koitieren' 
SSA (I: 384); in EES nicht behandelt

3 wot. koipa 'Bein, Fuß'

fi. koipi '(Hinter)bein, Oberschenkel, Keule'

EES $(171)^{84}$

4 wot. koira, koiraz, koiruz 'Hund'

fi. koira 'Hund'

vll. ursprünglich Ableitung vom Stamm *koj(e) (SSA I: 385); ural. (EES 168)

UEW (I: 168f.): $k o j(e)-r a$ (ural.)

5 wot. oigata, oikkia 'ächzen, stöhnen; klagen'

est. oiata 'ächzen, stöhnen'

EES (335)

6 wot. oikka 'schmal, schlank, dünn'

fi. hoikka 'schmal, schlank, dünn'

SSA (I: 169), in EES nicht behandelt

7 wot. oita ' pflegen, hüten; führen, sparen'

fi. hoitaa 'pflegen, hüten; führen'

SSA (I: 169), EES (77f.)

8 wot. poika 'Sohn, Junge; kleiner Junge'

fi. poika 'Sohn; Junge'

? Urstamm * ројз (SSA II: 385); fiugr. (EES 378)

fiugr. pojka (UEW I: 390)

9 wot. poizag, poizek, poiz(a) 'weg'

fi. pois 'weg'

SSA (II: 386); in EES nicht behandelt

10 wot. roittu 'Kram, Trödel, Abfall'

fi. roitto 'Abfall; Fetzen, Lumpen'

SSA (III: 88), in EES nicht behandelt

11 wot. (Tsv.) toim(i) 'Erkundigung, Anfrage, Kenntniserhalt, Erklärung, Erläuterung'

fi. toimi 'Beschäftigung, Arbeit [...]'

Urstamm *toj(3)- (SSA III: 304), EES (535)

\footnotetext{
${ }^{84}$ In EES (171) stellt man die wotische Entsprechung mit der finnischen zusammen, VKS (448) nennt dagegen lediglich den Dialekt Luuditsa sowie eine Form nach TsVETKOV.
} 
1: Warum der Lautwandel nicht abläuft, kann nicht begründet werden. KETTUNEN (VKÄ 135) nimmt Fremdeinfluss an, wenn $*$ oi bewahrt wird.

2-4, 6-8: Der Lautwandel tritt nicht ein, in der zweiten Silbe dieser Belege liegt $a$ vor.

5: Der Beleg wird als onomatopoetisch beeinflusst definiert.

9: Die Folgesilbe des Belegs kann nicht eindeutig bestimmt werden, vgl. auch die Diskussion um est. (dial.) poiss 'weg' in Kapitel 5.5.3.2.1.

10: Der Beleg gilt als deskriptiv, eine lautliche Schwankung kann daher vorliegen.

11: Der Beleg ist nach TsVETKOV notiert und somit nicht aussagekräftig.

Aus dem Korpus zählen noch zwei weitere Belege zur Gegenprobe:

185 wot. koivu 'Birke'

fi. koivu 'Birke'

205 wot. roivaz, '(Flachs-, Stroh-) Bündel'

fi. roivas 'Hanf- oder Flachsbündel, -garbe'

(185) Eine Begründung für das Bewahren von *oi konnte nicht ermittelt werden.

(205) Bei diesem Beleg ist ebenso nicht klar, warum der Lautwandel nicht eintritt. Auffällig ist die auf $a$ lautende Folgesilbe.

Betrachtet man die einschlägigen Belege im Vergleich zur Gegenprobe, müssen auch im Wotischen die Bedingungen für den Lautwandel um eine weitere ergänzt werden: Sofern in der Folgesilbe $a$ auftritt, wird *oi oftmals bewahrt. Der Lautwandel ist somit nicht lautgesetzlich. KETTUNEN beschreibt auch für das Wotische eine Zweigeteiltheit bei oi oi , ergänzt jedoch, dass er für das Bewahren von *oi Fremdeinfluss annimmt (VKÄ 135).

\subsection{Livisch}

Im Livischen ist der Lautwandel *oi > $\tilde{o} i$ mit nur insgesamt sechs Belegen am schwächsten von allen Sprachen vertreten.

mit zweiter Silbe, die auf $* a$ zurückgeht:

(210) liv. seitz 'jm. etw. vorwerfen',

(221) liv. võidõ 'können'; 
mit zweiter Silbe, die auf *e zurückgeht:

(199) liv. öigi 'recht, richtig',

(219) liv. võ̃idag ,Butter“;

mit zweiter Silbe, die auf $* u$ zurückgeht:

(185) liv. kõuvõ 'Birke';

mit zweiter Silbe, die nicht bestimmbar ist:

(220) liv. vęih '(Interjektion, z.B. zum Schrecken)'.

\section{ANMERKUNGEN}

(185) Eine Entwicklung des zweiten Diphthongbestandteils * $i>u$ läuft vor $v$ ab (PoSTI 1942: 32f.). Ob dieser Lautwandel älter ist als $*_{o}>\tilde{o}$ des ersten Bestandteils, kann nicht näher bestimmt werden, da das Material dies nicht hergibt.

(199) Posti (1942: 33) setzt für diesen Beleg eine estnische Entlehnung an, da nach seiner Auffassung die Entwicklung im Livischen lautgesetzlich anders hätte verlaufen müssen: livO. *voìgdô, livW. *vºìgdâ). SSA (II: 260) bestätigt diese Entlehnung.

(210) In den etymologischen Wörterbüchern wird eine onomatopoetisch beeinflusste Form angesetzt (vgl. SSA (III: 193) und EES (493f.)), was wohl die Ursache für die Entwicklung ist. PosTi behandelt den Beleg in seinen Ausführungen nicht.

(219) Angesetzt wird ein Urstamm fiugr. woje 'Fett, Fettstoff' (UEW I: 578) und somit ein *e in der früheren Vertretung der zweiten Silbe. Der Lautwandel *oi > $\tilde{o} i$ sei laut Posti (1942: 33) vor oder nach $v$ regelmäßig.

(220, 221) Hier ist abermals der regelmäßige Lautwandel *oi > $\tilde{o} i$ vor oder nach $v$ anzuführen (Posti 1942: 33).

\section{GEGENPROBE}

Während im Korpus keine Belege auftreten, die für eine Zusammenstellung hier von Relevanz sind, konnten anhand der etymologischen Wörterbücher drei livische Wörter in diesem Kontext ermittelt werden. Die geringe Anzahl an Belegen hängt bei diesem Lautwandel abermals mit 
der eigenständigen Entwicklung des Livischen zusammen. POSTI (1942: 31f.) setzt beispielsweise eine allgemeingültige Vertretung $u ̀ o i ~{ }^{u} o \grave{~ f u ̈ r ~ * o i ~ a n . ~}$

$1 \quad$ liv. kuoi, koi 'Morgenrot'

fi. koi 'Morgendämmerung; Osten'

SSA (I: 383), EES (171)

fiugr. koje 'Morgenröte' (UEW I: 167)

2 liv. voido 'festhalten; beschützen; bewahren; bewachen'

fi. hoitaa 'für jemanden sorgen, jemanden pflegen'

SSA (I: 169), EES (77f.)

$3 \quad$ liv. (v)oik, vōiti 'dünn'

fi. hoikka 'schmal, schlank, dünn'

SSA (I: 169); in EES nicht behandelt

1: Vgl. Estnisch. Unklar ist, warum der Lautwandel in der ersten Silbe auch im Livischen in der Variante liv. koi nicht eintritt. In liv. kuoi wird er durch die regelmäßige Entwicklung *oi > uoi verhindert.

2, 3: Der Grund dafür, dass der Lautwandel hier nicht eintritt, ist ebenso wenig erkennbar. Für die Folgesilbe kann wohl *a angesetzt werden.

Im Livischen ist das Bild nicht sonderlich eindeutig. Ansetzbar ist wohl dennoch, dass der Lautwandel bei den wenigen Belegen, so er nicht durch spracheigene Entwicklungen verhindert wurde, unabhängig der zweiten Silbe eintritt. Bei * $a$ in dieser Position wird die alte Vertretung gelegentlich bewahrt. POSTI (1942: 31f.) setzt in seiner Lautlehre die folgende Vertretung für den Diphthong *oi an: *oi sei im Allgemeinen durch ùoi ${ }^{u}$ oì vertreten, im Anlaut durch vòi voì (Ost) bzw. vùoi $\sim v^{u}$ ò (West). ${ }^{85}$

\subsection{Südestnisch}

Dem Lautwandel $* o i>\tilde{o} i$ unterliegen im Südestnischen die nachfolgenden Belege: mit zweiter Silbe, die auf *a zurückgeht:

(193) estS. .mõisa 'Gut, Landgut',

\footnotetext{
${ }^{85} \mathrm{Zu}$ Abweichungen von dieser Vertretung vgl. POSTI (1942: 31ff.).
} 
(196) estS. nõid, gen. nõia 'Zauberer, Hexe',

(205) estS. rõivas, gen. .rõiva 'Kleid, Kleidung',

(210) estS. .sõitma, II. Infinitiv .sõitaq 'fahren',

(221) estS. .võima, II. Infinitiv .võidaq 'können';

mit zweiter Silbe, die auf $* e$ zurückgeht:

(188) estS. lõim', gen. lõimõ 'Weberkette',

(199) estS. .õigõ 'recht, richtig',

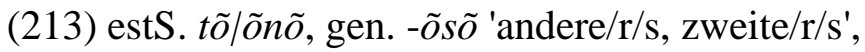

(219) estS. võid, gen. võiu'Butter';

mit zweiter Silbe, die auf *u zurückgeht:

(185) estS. kõiv, gen. - $u$ 'Birke'.

\section{ANMERKUNGEN}

(213) Der zweite Bestandteil des Diphthongs wird an den ersten assimiliert (KEEM 2002: 36).

(219) Ein Urstamm fiugr. woje 'Fett, Fettstoff' (UEW I: 578) wird angesetzt, sodass $* e$ der Vokal der zweiten Silbe gewesen sein muss.

Abermals kann anhand der gemischten Verteilung der Belege konstatiert werden, dass der Lautwandel $* o i>\tilde{o} i$ wohl unabhängig von der Folgesilbe auftritt.

\section{GEGENPROBE}

Für die Gegenprobe konnten die nachfolgend angeführten Belege ermittelt werden. Im Korpus lagen keine relevanten Ergänzungen vor.

1 estS. .hoitma, .hoitaq 'festhalten; beschützen; bewahren; bewachen'

fi. hoitaa 'für jemanden sorgen, jemanden pflegen'

SSA (I: 169), EES (77f.)

2 estS. koi 'Abendrot'

fi. koi 'Morgendämmerung; Osten'

Laut SSA (I: 383) noch in syrj. und wog. belegt; fiugr. oder ural. (EES 171)

fiugr. koje 'Morgenröte' (UEW I: 167) 
3 estS. koib, gen. koiva '(Hinter)bein, Oberschenkel, Keule, Schenkel; Rand des Fells mit Beinteilen, Überbleibsel des Leders'

fi. koipi '(Hinter)bein, Oberschenkel, Keule'

SSA (I: 384), EES (171)

4 estS. loid, gen. loiu 'matt, träge, flau'

fi. dial. loitia '(müßig) liegen, faulenzen'

SSA (II: 88), EES (246)

5 estS. .oigama, ojadaq 'ächzen, stöhnen'

wot. oigata, oikkia 'ächzen, stöhnen; klagen'

EES (335)

6 estS. oinas, gen. .oina 'Schafbock, Widder, Hammel'

fi. oinas 'Hammel, Schafbock'

< balt. (SSA II: 261, EES 335)

7 estS. .roiduma, .roidudaq 'ermatten, ermüden'

fi. dial. roitua 'ermüden, schwach werden'

EES (433), in SSA nicht behandelt

8 estS. roisk, gen. roiso 'Moder, Fäulnis; Gesindel; Verderben, Verschwenden'

fi. dial. roiska 'alte, elende Maschine' (SSA: 'Rute, Peitsche, Reisig')

EES (434), in SSA (III: 88) nicht zusammengestellt

1, 3, 6: Der Grund für das Bewahren von $* o i$ ist unklar. Auffällig ist das Auftreten von $* a$ in der Folgesilbe.

2: Warum der Lautwandel in diesem Beleg nicht eintritt, kann nicht geklärt werden.

4, 5, 7, 8: Der deskriptive bzw. onomatopoetische Wortcharakter ist Grund für die Abweichung.

Nach Betrachtung der Gegenbeispiele muss die Aussage über den Lautwandel *oi $>\tilde{o} i$ auch für das Südestnische ergänzt werden. Von der zweiten Silbe unabhängig tritt ein Lautwandel * $o i>$ $\tilde{o} i$ ein. Liegt in dieser Position $* a$ vor, bleibt $* o i$ häufig erhalten. Da die Bedingungen aufgrund der Verschiedenheit der Belege nicht konkretisiert werden können, kann der Lautwandel nicht als lautgesetzlich bezeichnet werden. 
Dieses Kapitel widmet sich den Sonderfällen. Insgesamt fallen fünf einschlägige Belege in diese Kategorie. Sie sind nicht immer in allen drei Sprachen auffällig oder abweichend, weisen aber in jeweils einer eine unerwartete Form auf.

(222) est. lõo(ke) 'Lerche'

Der Beleg geht auf urfi. *leivo (LÄGLOS II: 190) zurück. Vor labialen Vokalen schwindet $v$ häufig (EKÄ 116). Der Schwund von $i$ bei Diphthongen tritt im Estnischen normalerweise nur in nichtersten Silben auf, vgl. z.B. est. soolane 'salzig' fi. suolainen id. (ebd. 163). Der Grund für Synkope von $i$ kann mangels weiterer Anhaltspunkte nicht ermittelt werden.

\section{(223) wot. $\tilde{o} \tilde{o} k k^{\prime}$ 'Distel'}

Die Bedingungen für die Längung der ersten Silbe sind unklar; sie wirkt unmotiviert, vgl. auch wot. Varianten ohtain, ohtajain, ohtiain (EES 334) sowie Entsprechungen in anderen osfi. Sprachen: fi. ohdake 'Distel' est. ohakas id.

(224) wot. nõisa 'anfangen, beginnen'

KETTUNEN (VKÄ 137) zufolge stamme das $i$ im Wotischen ursprünglich aus einer Form, in deren zweiter Silbe regulär $i$ auftritt. Daraufhin sei eine Assimilation eingetreten und das Paradigma habe sich an diese Form angepasst. Bei der livischen Entsprechung spricht POSTI (1942: 13) von „Verschiedenheiten“ in der ersten Silbe, die älter sein können als das Späturfinnische, definiert diese aber nicht genauer. Die Verhältnisse sind weiterhin unklar. Der Ausfall von $u$ im Südestnischen scheint unbegründet und wird in EKÄ und von KEEM (2002) nicht behandelt.

(225) est. sõ̃rd, gen. - $u$ 'Schwende'

Der rekonstruierte Stamm ist urfi. *sorta- (LÄGLOS III: 248); die Bedingungen für die Längung der ersten Silbe sind unklar. Es ist von einer unmotivierten Entwicklung auszugehen.

(226) est. tõotada 'versprechen, geloben'

Die Verhältnisse sind ähnlich wie die des Belegs (222). 
Neben den beiden stark betroffenen Lauten $* e$ und $*_{o}$, zeigen sich auch bei vereinzelten Belegen, die in der ersten Silbe auf andere Vokale zurückgehen, Entwicklungen, die zu o geführt haben. Auf diese soll nachfolgend eingegangen werden. Sofern Anhaltspunkte zur Aufklärung einzelner Belege vorliegen, wird darauf hingewiesen.

\subsubsection{Kurzvokale}

$* a>\tilde{o}$

(42) est. lõng, gen. - a 'Garn, Faden' wot. lõnka id.

(103) est. põleda 'brennen' wot. põlõa id.

(127) est. sõna 'Wort' wot. sõna id. liv. sõnā id. estS. sõna id.

$*_{i}>\tilde{o}$

(105) liv. põnktõ 'strammen'

$* u>\tilde{o}$

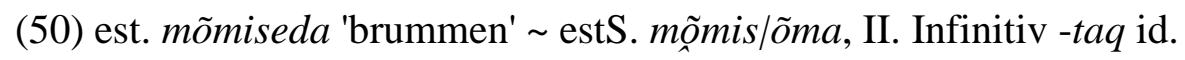

(64) est. nõmm, gen. - $e$ 'Heide' wot. nõmmi, gen. nõmmõ $\tilde{\text { id. } ~ \sim ~ e s t S . ~ n o ̃ m m ~ g e n . ~}-e$ id.

$*_{\ddot{o}}>\tilde{\sigma}$

(45) liv. mõgā 'Geräusch, Dröhnen, Gedröhne'

$* \ddot{u}>\tilde{o}$

(11) liv. jõvā 'gut',

(118) liv. rõzā 'Reuse',

(143) liv. tỗlza 'stumpf (Waffe, Streitwaffe), ranzig geworden'

(150) liv. tõ 'v 'Stamm',

(151) liv. tõ $v \bar{a}$ 'tief',

(11, 150, 151) Posti (1942: 15f.) setzt einen regelmäßigen Lautwandel $* \ddot{u}>\tilde{o}$ vor $v$ an. Die Bedingungen dafür seien jedoch nicht geklärt. 
(118) Kettunen (LW 335) geht von einem notierten Sprachfehler aus. Der livische Sprecher, von dem das Wort aufgezeichnet wurde, konnte den Laut $\ddot{u}$ nicht aussprechend und habe stattdessen $\tilde{o}$ in der ersten Silbe verwendet.

Unbekannter Ausgangslaut

(36) est. krõmpsuda 'krachen, knacken' liv. krẹmpš 'Kruste, Rösch, ein essbarer Pilz'

(116) est. rõkkata 'laut jauchzen, gellen, schallen' liv. rõkkõ 'sagen, ansprechen' estS. rõkkama 'laut jauchzen, gellen, schallen'

\subsubsection{Langvokale}

Unbekannter Ausgangslaut

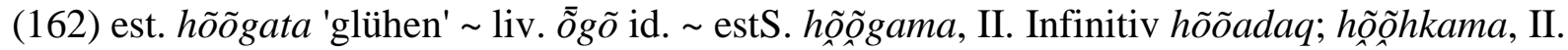
Infinitiv hõohadaq id.

(164) est. kõom, gen. - $a$ 'Kopfschuppen' liv. kõ̃ma 'Knorpelchen' estS. koomõq, gen. kuumõ; kohmõq, gen. .kohmõ 'Kopfschuppen'

(162) Da der Lautwandel bei den Langvokalen nachweislich nur bei *ee eintritt, ist dieses wohl als historische Vertretung ansetzbar.

(164) Zwar wäre auch hier *ee erwartbar, die südestnische Form wirft aber Fragen auf, die nicht beantwortet werden können, weil keine Belege unter ähnlichen Bedingungen vorkommen.

\subsubsection{Diphthonge}

$* a u>\overline{\tilde{O}}$

(195) liv. mȭka 'Wurst, Würstchen'

$* i u>\tilde{o u}$

(180) liv. jõugõ 'Sand'

(191) liv. lõuglõ 'gleiten; stolpern, straucheln',

(217) liv. tõurõz 'teuer' 
Nach PostI (1942: 38f.) ist ansetzbar, dass ein Lautwandel $* i>\tilde{o}$ durch $u$ als zweiten Bestandteil des Diphthongs ausgelöst wurde. ${ }^{86}$ POSTI geht sogar so weit, die Vertretung als lautgesetzlich zu bezeichnen. Wörter, die das *iu im Livischen bewahrt haben, seien ihm zufolge Onomatopoetika, z.B. liv. kiùkà 'heulen, winseln', oder, wie im Fall von liv. kiùzâ 'peinigen', möglicherweise aus dem Estnischen entlehnt (ebd. 39).

$* a i>\tilde{o} i$

(183) est. kõi|k, gen. -ge 'alle' wot. kõikki, gen. kõikõõ id. estS. kõ $\mid i k$ ', gen. -gõ id.

(218) est. või 'oder' wot. või id. liv. või id. | estS. vai id.

$* u i>\tilde{o} i$

(194) est. mõista 'verstehen, begreifen' wot. mõissaa id. estS. .mõistma, II. Infinitiv .mõistaq id.

(211) liv. sõitõ 'können, vermögen'

\subsection{Zusammenfassung der Analyse}

Die Analyse hat die Verhältnisse zu den Lautwandeln in allen vier untersuchten Sprachen dargelegt und an notwendigen Stellen diskutiert und interpretiert. Anhand der Erkenntnisse lassen sich nun für die einzelnen Entwicklungen Lautwandel definieren, die jedoch nicht immer lautgesetzlich sind. Komprimiert lassen sich die Ergebnisse wie folgt zusammenfassen:

\section{KURZVOKALE}

\section{1) $* e>\tilde{o}$ bei Hintervokal in der Folgesilbe.}

Die Regel ist gültig und kann wohl als lautgesetzlich festgehalten werden. Die vermeintlichen Ausnahmen, die im Laufe der Analyse ermittelt wurden, konnten in allen Sprachen erklärt werden. Lediglich im Livischen und Südestnischen gab es eine kaum nennenswerte Zahl Abweichungen. Die Gegenproben ergaben zwar vereinzelte Unklarheiten, die jedoch in einer derart marginalen Anzahl vorliegen, sodass sie eine GesetzmäBigkeit nicht entkräften können.

\footnotetext{
${ }^{86} \mathrm{Zu}$ vereinzelten Abweichungen von dieser Entwicklung s. PosTI (1942: 39).
} 


\section{2) $* o>\tilde{o}$ bei Vordervokal oder $* a$ in der Folgesilbe.}

Die meisten Belege, die unter diesen Lautwandel fallen, verhalten sich regelmäßig. Für die Abweichungen gibt es unterschiedliche Erklärungen, wie beispielsweise Onomatopoetika. Es verbleiben dabei jedoch häufiger Belege, die das *o in der ersten Silbe bewahren und nicht erklärt werden können, sodass hier keine Lautgesetzlichkeit vorliegt. Das Livische vollzieht den Lautwandel meist nicht und nimmt aufgrund seiner sprachinternen Entwicklungen eine Sonderstellung ein.

Da Lautwandel bei weiteren Vokalen in der ersten Silbe nur in geringer Anzahl auftreten, kann für diese keine Regel festgelegt werden.

\section{LANGVOKALE}

1) *ee $>\tilde{\boldsymbol{o}} \tilde{o}$ bei Hintervokal in der Folgesilbe.

Die untersuchten Entsprechungen dieses Lautwandels verhalten sich regelmäßig. Die einzige augenscheinliche Abweichung konnte durch die Betrachtung geklärt werden. Zwar haben die Gegenproben auch hier vereinzelte Belege mit Abweichung ergeben, jedoch in derart geringer Anzahl, dass auch hier das Lautgesetz nicht entkräftet wird.

\section{2) Ein Lautwandel *oo > $\tilde{o} \tilde{o}$ tritt nicht auf.}

Für einen derartigen Lautwandel ließen sich anhand des Korpus keine Beweise finden.

\section{3) Weitere Lautwandel treten nicht auf.}

Ein Lautwandel bei anderen Langvokalen konnte ebenso wenig festgestellt werden.

\section{DIPHTHONGE}

1) Der Lautwandel *ou > $\tilde{o u}$ ist lautgesetzlich.

Eine Gegenprobe hat dieses Lautgesetz nicht entkräften können.

\section{2) Der Lautwandel *eu > ou ist lautgesetzlich.}

Der Lautwandel liegt nur in einer geringen Anzahl an Belegen vor, eine Gegenprobe hat mitunter keine Abweichungen ergeben und die Annahme der Regelmäßigkeit des Lautwandels bestätigt. 
3) $* e i>\tilde{o} i$, wenn in der zweiten Silbe ein Hintervokal vorliegt.

Die Verbreitung dieses Lautwandels ist insgesamt nicht sonderlich hoch. Die Regel lässt sich anhand der diskutierten Belege bestätigen, eine Gegenprobe konnte auch hier die These nicht entkräften, da die meisten Abweichungen aufgeklärt werden konnten. Im Livischen liegt dieser Lautwandel nicht vor.

4) Für den Diphthong *oi gilt, dass der Lautwandel *oi > $\tilde{i} i$ unabhängig von der zweiten Silbe eintritt. Liegt in dieser Position $a$ vor, bleibt *oi oftmals bewahrt. Für das Livische lässt sich festhalten, dass der Diphthong durch ùoi "oì vertreten ist, im Anlaut durch vòi vò̀ (Ost) bzw. vùoi $\sim v^{u} o \grave{~(W e s t) . ~}$

Im Hinblick auf das Estnische, Wotische und Südestnische lässt sich der Lautwandel bestätigen und ebenso die bereits mehrfach genannte Zweiteilung der Entwicklung von *oi festhalten. Tritt $a$ in der zweiten Silbe auf, kommt es zu einem ambivalenten Verhältnis, in dem der Lautwandel bei einigen Belegen eintritt, bei anderen dagegen nicht. Die Regel konnte in diesem Rahmen nicht genauer eingeschränkt werden, da die Belege keinem eindeutigen Muster folgen. Die Entwicklung ist folglich nicht lautgesetzlich.

Die Analyse hat ferner gezeigt, dass das Livische den Lautwandel nur in einem sehr geringen Umfang vollzieht. Stattdessen liegen sprachinterne Entwicklungen vor, die einen Lautwandel zu $\tilde{o}$ verhindert haben. Damit nimmt das Livische auch bei den Diphthongen im Vergleich zu den anderen Sprachen eine Sonderstellung ein.

5) Die Diphthonge *ai und *ui werden in den Sprachen normalerweise bewahrt.

Es liegen kaum Belege vor, die auf diese Laute zurückgehen und einem Lautwandel unterliegen. Von Einzelfällen ist auszugehen.

6) Im Estnischen entwickelt sich das $i$ als zweiter Bestandteil des Diphthongs unter bestimmten Bedingungen zu $e$, nicht jedoch in für den Lautwandel zu $\tilde{o}(i)$ einschlägigen Belegen.

Wörter, in denen der zweite Bestandteil des Diphthongs diese Entwicklung durchläuft, sind für die Lautwandel zu õ im ersten Bestandteil nicht einschlägig. 


\section{SONDERFÄLLE}

Die Anzahl der Sonderfälle ist in diesem Korpus gering und aufgrund ihres speziellen Status und dem Unterschied in der Art der Abweichungen wurden keine Regeln für sie festgelegt. Sie sind als Einzelfälle zu werten.

\section{9}

\section{Belege mit weiterem Diskussionsbedarf}

Die Belege, die für die im Rahmen der Analyse keine Lösungsansätze gefunden werden konnten, werden in diesem Kapitel zusammengefasst. Obgleich viele der genannten Belege Raum für Fragen lassen, so haben die Antworten darauf nicht immer direkte Auswirkungen auf den Lautwandel. Für die Entwicklung * $e>\tilde{o}$ ist zwar wesentlich, dass ein Hintervokal in der zweiten Silbe vorliegt, die Qualität dieses Lautes spielt jedoch für die Entwicklung nur eine untergeordnete Rolle.

\section{Unbestimmbarer Hintervokal als Stammvokal}

In einigen Belegen konnte zwar ein Hintervokal als Stammvokal angesetzt werden, es war jedoch nicht möglich, diesen genauer zu bestimmen.

- Kein etymologisch sicherer Nachweis für genaue Bestimmung des Hintervokals (55) est. mõtelda 'denken, nachdenken' $\sim$ wot. mõtõlla id. $\sim$ liv. mõtlõ id. $\sim$ estS. .mõtlõma, II. Infinitiv mõtõldaq id. < urfi. *mettV- (LÄGLOS II: 281)

- Unklarer Lehngeber (186) est. kõu, gen. - e 'Donner' estS. kõvve id. < balt. (vgl. lit. kaũkas 'zwergartiger Schutzgeist des Landes oder des Hauses, Wichtel' und lett. kauks 'Wichtel') oder < indoeur. *kouko- (SSA I: 414)

\section{Unbestimmbarer Stammvokal}

In vielen Fällen konnte der Stammvokal weder ermittelt noch anhand der Belege und ihrer Etymologien weiter eingegrenzt werden, sodass ihr Status in der Hinsicht ungeklärt bleibt.

- Ungesicherter Stammvokal (142) est. tõnguda 'wühlen' wot. tõygata id. (? Stamm auf $a$ ) (201) est. oud(ne) 'unheimlich' < ? *outo

- Unbekannter Stammvokal, Belege sind Onomatopoetika/Deskriptiva (10) est. jõlkuda 'hin und her gehen' estS. .jõlkma, II. Infinitiv jõlkuq id. 
(47) est. mõksuda 'zusammenschlagen, zusammenstoßen [...]'

(136) est. sõtkuda 'treten, kneten' wot. sõtkoa/-ua id. estS. .sõkma, II. Infinitiv sõkkuq id.

- Unbekannter Stammvokal

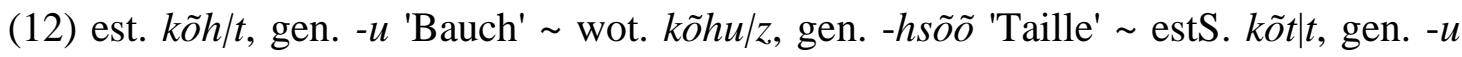
'Bauch'< fi.-perm. kokt3 'Bauch' (UEW II: 670)

(36) est. krõmpsuda 'krachen, knacken' liv. krẹmpš 'Kruste, Rösch, ein essbarer Pilz'

(46) est. mõjuda 'beeinflussen, wirken' estS. mõo|ma, II. Infinitiv -daq id.

(75) est. ôhuke 'dünn' wot. ôhuD id. ? <*oha-

(139) wot. tõhtia 'dürfen, können' < urfi. *tošt-

(145) est. tõrjuda 'abwehren, verdrängen' estS. tõrjuda id.

(203) est. põimida 'flechten, winden' wot. põimia 'sammeln, pflücken'

\section{Unmotivierter Lautwandel}

An einigen Stellen scheint das Aufkommen des neuen Lautes unmotiviert. Die Entwicklung kann anhand der Daten nicht begründet werden.

(42) est. lõng, gen. - $a$ 'Garn, Faden' wot. lõyka id.

(45) liv. mõg ā 'Geräusch, Dröhnen, Gedröhne'

(50) est. mõmiseda 'brummen' estS. mõmis|õma, II. Infinitiv -taq id.

(64) est. nõmm, gen. - $e$ 'Heide' wot. nõmmi, gen. nõmmõ $\tilde{\text { id. } ~ \sim ~ e s t S . ~ n o ̃ m m ~ g e n . ~}-e$ id.

(81) est. $\tilde{o l} \mid u$, gen. -le 'Bier' wot. õlu|D, gen. - $u$ 'id.' < urfi. *olut

(77) liv. ó'ldzõ 'hell, grell, klar' < *hele- $\delta A$; frühe Synkope von *e?

(103) est. põleda 'brennen' wot. põlõa id.

(105) liv. põnktõ 'strammen'

(127) est. sõna 'Wort' wot. sõna id. liv. sõnā id. estS. sõna id.

(143) liv. tỗlza 'stumpf (Waffe, Streitwaffe), ranzig geworden'

(146) liv. võttõ 'nehmen'

(195) liv. $m \overline{\tilde{o} k a}$ 'Wurst, Würstchen'

(183) est. kõi|k, gen. -ge 'alle' wot. kõikki, gen. kõikõõ id. estS. kõ |ik', gen. -gõ id.

(194) est. mõista 'verstehen, begreifen' wot. mõissaa id. estS. .mõistma, II. Infinitiv .mõistaq id.

(211) liv. sõitõ 'können, vermögen'

(218) est. või 'oder' wot. või id. liv. või id. 


\section{Unklarer Wortstatus}

Einige Belege sind etymologisch nicht eindeutig. Im Kontext dieser Untersuchung konnte keine Klarheit geschaffen werden.

(56) liv. mõtsā 'Wald' estS. mõts, gen. mõtsa id. < fiugr. mećä 'Rand, Seite von etwas' (UEW I: 269f.); ? unterschiedliche Entlehnung (vgl. Kapitel 5.3.2.)

(174) liv. sēmda 'Milch' ? deskriptiv, ? Kindersprache, ? < lett.

(190) liv. lōkandõks 'Eisloch', unklare Etymologie

(211) liv. sõitõ 'können, vermögen', ? Doppelung mit (210)

\section{Unbekannte Laute der ersten Silbe}

Für vereinzelte Belege konnte ferner die erste Silbe nicht gesichert ermittelt werden, da ihre Etymologien unklar sind.

- Unbekannte Laute ohne Lösungsansatz:

(36) est. krõmpsuda 'krachen, knacken' liv. krẹmpš 'Kruste, Rösch, ein essbarer Pilz' (116) est. rõkkata 'laut jauchzen, gellen, schallen' liv. rõkkõ 'sagen, ansprechen' estS. rõkkama 'laut jauchzen, gellen, schallen'

(164) est. kõ̃m, gen. - a 'Kopfschuppen' liv. kõma 'Knorpelchen' estS. koomõq, gen. kuumõ; kohmõq, gen. .kohmõ 'Kopfschuppen'

- Unbekannte Laute der ersten Silbe; aufgrund der Regelmäßigkeit des Lautwandels hier wohl *ee:

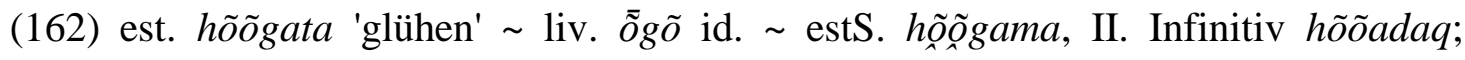
hõ õ hkama, II. Infinitiv hõôhadaq id. 
6. Schlussfolgerungen aus der Analyse

Welche Schlussfolgerungen ergeben sich aus der Analyse des Lautwandels in den untersuchten Sprachen? Dieser Frage wird im letzten Teil dieser Untersuchung nachgegangen. Neben Überlegungen zur Herausbildung des neuen Lautes $\tilde{o}$ und zum zeitlichen Ablauf einzelner, für die Entwicklung der ersten Silbe relevanter Lautwandel, die auf der vorausgegangenen Analyse begründet sind, werden auch Überlegungen zum Ablauf der Ausbreitung der Entwicklungen unternommen.

\subsection{Allgemeine Bemerkungen}

Im Hinblick auf die zahlenmäßige Verteilung des Lautwandels auf die drei Kategorien Kurzvokale, Langvokale und Diphthonge zeichnet sich zunächst ein erwartbares Bild ab: Die Kurzvokale liegen in der ersten Silbe ganz allgemein und ohne Berücksichtigung der einzelnen Lautwandel betrachtet in den Einzelsprachen am häufigsten vor (161 der 226 Belege im Korpus, unabhängig von ihrer Einschlägigkeit in den Einzelsprachen).

Die verschieden große Anzahl der einschlägigen Vertretungen in den betroffenen Einzelsprachen deutet auf unterschiedliche Entwicklungen hin. Das Estnische, Wotische und Südestnische sind noch ähnlichen häufig von der Entstehung und Ausbreitung von $\tilde{o}$ betroffen, während das Livische ein ganz anderes Bild zeigt. Dass das Livische den neuen Laut nur teilweise in Gebrauch nimmt, aber stattdessen meist anderen Lautwandeln in der ersten Silbe unterliegt, spricht für die eigenständige Entwicklung des Vokalismus dieser Sprache: Die Analyse des Sprachmaterials hat gezeigt, dass interne Gegebenheiten eine Ausdehnung von $\tilde{o}$ verhindert haben. Diese Tatsache unterstreicht deutlich, dass das Livische sich insgesamt sehr eigenständig und vor allem weitestgehend unabhängig von den anderen drei Sprachen entwickelt haben muss (vgl. dazu auch das anschließende Kapitel 6.3 Zur Ausbreitung und zum Alter des Lautwandels). Deutlich wird dies besonders bei der Entwicklung von * $o$.

Unabhängig von der Kategorie sind meist die Laute * $e$ und $*_{o}$ von Lautwandel mit $\tilde{o}$ betroffen, während die anderen Vokale diesen Entwicklungen in allen Sprachen nur marginal unterliegen. In dieser Hinsicht sind die untersuchten Sprachen ähnlich ausgeprägt. Die Gründe dafür, dass ein Lautwandel bei einigen Vokalen nicht eintritt, hängen mit der lautlichen Qualität der einzelnen Laute zusammen. Betrachtet man diese, so lässt sich feststellen, dass $e$ und $o$ ebenso 
mittelhohe Vokale sind, wie der neue Laut $\tilde{o}$. Der Vokal $a$ ist tief bzw. offen, die Vokale $i$ und $u$ sind hoch bzw. geschlossen. Sie stehen also auf einer anderen Artikulationsebene als $\tilde{o}$. Zur Veranschaulichung dient ein Blick auf den estnischen Vokalismus, der bereits zu Beginn vorgestellt wurde (VIITSO 2003: 20; ASU \& TERAS 2009: 368):

\begin{tabular}{ccccc} 
& \multicolumn{2}{c}{ Vordervokale } & \multicolumn{2}{c}{ Hintervokale } \\
& Illabial & Labial & Illabial & Labial \\
Hoch oder geschlossen & $i[\mathrm{i}]$ & $\ddot{u}[\mathrm{u}]$ & & $u[\mathrm{u}]$ \\
Mittelhoch & $e[\mathrm{e}]$ & $\ddot{o}[\varnothing]$ & $\tilde{o}[\gamma]$ & $o[\mathrm{o}]$ \\
Tief oder offen & $\ddot{a}[\mathfrak{x}]$ & & $a[\mathrm{a}]$ &
\end{tabular}

Es wirkt vor diesem Hintergrund wahrscheinlich, dass die unterschiedlichen Voraussetzungen in der Artikulationshöhe der Vokale die Verbreitung des neuen Lautes beeinflusst haben. Der Vordervokal $e$ ist illabial, $o$ ist ein labialer Hintervokal. Beide sind jedoch ihrer unterschiedlichen Eigenschaften zum Trotz für den Lautwandel sehr empfänglich. Wie ist das Verhältnis zu interpretieren? Zwei für die Verbreitung von õ maßgebliche Entwicklungen haben sich in der Analyse gezeigt:

1. $* e>\tilde{o}$ bei hintervokalischer Folgesilbe

2. $* o>\tilde{o}$
a. bei vordervokalischer Folgesilbe
b. bei $a$ in der Folgesilbe

Alle relevanten Lautwandel sind eine Form der Assimilation der historischen Vertretung der ersten an die Folgesilbe. Während $* e$ durch die Annäherung an die hintervokalische Folgesilbe velarisiert wird, wird ${ }^{*} o$ bei dieser Entwicklung als natürliche Konsequenz der Annäherung an die Laute der Folgesilbe entlabialisiert. Dies ist insofern bemerkenswert, als die infragestehenden Vokale $e, i$ und $a$ allesamt selbst illabial sind. Daraus lässt sich ableiten, dass der Lautwandel $* o>\tilde{o}$ wohl eintritt, wenn in der Folgesilbe ein illabialer Vokal steht.

Die Lautwandel sind nicht gänzlich unabhängig voneinander entstanden, sondern stehen vielmehr in einem Zusammenhang, der auch chronologisch bedingt ist. Wie im wissenschaftsgeschichtlichen Kontext erläutert, schrieb schon ITKONEN (1945: 178f.), dass der Lautwandel 
wahrscheinlich aus einem leicht velarisierten * $e$ entstanden sei. Die Annahme wird anhand dieser Untersuchung bestätigt. Da ein ungerundeter Hintervokal $\tilde{o}$ folglich durch die Entwicklung $*_{e}>\tilde{o}$ bereits vorliegt, ist die Herausbildung des Lautwandels $*_{o}>\tilde{o}$ begünstigt. Nicht nur ist der neu gebildete Laut wie die den Lautwandel auslösenden illabial, er wird als Hintervokal auch weiter vorne artikuliert als andere Vokale dieser Kategorie (vgl. ASU \& TERAS 2009: 368f.). Die Entstehung des zweiten Lautwandels ist bedingt durch Ortsassimilation, also eine Assimilation der phonetischen Eigenschaften an einen anderen Artikulationsort (HALL 2011: 91): Der Artikulationsort von $* o$ wird an die illabialen Vordervokale oder das ebenso illabiale $a$ in der Folgesilbe angeglichen und dabei von $\tilde{o}$ aufgefangen, weil dieses der einzige Hintervokal auf gleicher Artikulationshöhe wie der Ausgangslaut ist. Die Entrundung des *o ist demzufolge sekundär.

Dass man insbesondere im Hinblick auf den Lautwandel $*_{o}>\tilde{o}$ sowie im Bereich der Diphthonge mit $* o$ als erstem Bestandteil auf viele Abweichungen trifft, kann nur bedingt als Unregelmäßigkeit ausgelegt werden, wie es z.B. ITKONEN (1945: 181f.) sieht. Es spricht vielmehr dafür, dass die Assimilation von ${ }^{*} o$ nicht abgeschlossen ist und nicht mehr sämtliche Wörter und Wortschichten durchlaufen hat. Der Lautwandel versiegte, das Vorkommen von $* o$ ist ambivalent: Belege unter gleichen Bedingungen, v.a. bei einem $a$ in zweiter Silbe, haben den Lautwandel $*_{o}>\tilde{o}$ mal vollzogen, mal jedoch den ursprünglichen Laut bewahrt, vgl. z.B. (34) est. kõva 'hart, fest, streng' wot. kõva id. estS. kõva id. vs. (13) est. koht, gen. koha 'Ort, Stelle' (jedoch wot. kõhta id.); (131) wot. sorkka 'Klaue' (jedoch est. sõr $\mid g$, gen. - $a$ id. estS. sõrg, gen. sõra id.). Dieses Phänomen wird auch als lexikalische Diffusion bezeichnet. Entgegen früherer Annahmen, dass immer alle Wörter einer Sprache gleichermaßen von einem Lautwandel erfasst werden, sofern sie die Bedingungen dafür erfüllen, muss vielmehr davon ausgegangen werden, dass es neben derartig regelmäßigen Lautwandeln auch Entwicklungen gibt, in denen nur ein Teil des Wortschatzes Veränderungen unterliegt (TRASK 2002: 289). TRASK (ebd.) beschreibt, dass ein Lautwandel einen wesentlichen Teil der Belege zwar verändert, nach einiger Zeit jedoch versiegt und einige Wörter dadurch ihre ursprüngliche Form behalten. Jene bezeichnet er als „,residue of the change“ - Rückstände oder Überreste des Lautwandels. Warum der Lautwandel $*_{o}>\tilde{o}$ nicht durch alle Formen läuft und einige Belege zu solchen „Überresten“"werden, ist trotz der eingänglichen Betrachtung und des Vergleichs nicht zu ermitteln. Das Versiegen ist somit wohl als grundlos zu bewerten. 
Ferner scheinen die Lautwandel irgendwann allgemein nicht mehr produktiv zu sein. Auch wenn die Bedingungen bei einigen Wörtern nach dem Ablauf weiterer Lautwandel erfüllt werden, dehnt sich $\tilde{o}$ nicht mehr aus. Darauf gibt es verschiedene Hinweise: Durch den Abbau der Vokalharmonie im Estnischen wurden in dieser Sprache eine Vielzahl regelkonformer Wortformen produziert. Ein Lautwandel ist jedoch nicht mehr nachträglich eingetreten, sodass die Wörter nun zunächst abweichend wirken, vgl. z.B. est. elada 'leben' ( fi. elää id.). Gleiches gilt für jüngere entlehnte Wörter, wie z.B. est. pedagoogika 'Pädagogik'. Würde die Produktivität der Lautwandel noch andauern, wäre zu erwarten, dass sie noch immer Wörter erfassen, die die Bedingungen für eine weitere Entwicklung der ersten Silbe später erfüllen oder die neu in die Sprache kommen. Ähnliche Tendenzen finden sich auch im Livischen. Einerseits gibt es Wörter aus älteren Sprachschichten, die die Bedingungen der ersten Silbe nachträglich erfüllen, einem der Lautwandel jedoch nicht unterliegen, z.B. liv. kejā 'Körper, Rumpf'. Ebenso finden sich jene, die jüngeren Alters sein müssen, jedoch ebenfalls * $e$ vorweisen, z.B. liv. mehānik 'Mechanik, Mechaniker'. Für das Wotische und Südestnische, die die Vokalharmonie nicht abgebaut haben, lassen sich ebenso Wörter jüngeren Alters identifizieren, die die Bedingungen für einen Lautwandel erfüllen, ihn jedoch nicht vollziehen, z.B. wot. mehaanikka 'Mechaniker', estS. tsello 'Cello' oder estS. démokraatia 'Demokratie'.

Auch hat sich $\tilde{o}$ in den Sprachen in der ersten Silbe nicht maßgeblich auf weitere Laute ausgedehnt, was einerseits der Qualität ebendieser geschuldet ist, andererseits nicht dafürspricht, dass der neue Laut, von seiner Ausdehnung auf $* e$ und $* o$ ausgenommen, sehr expansiv gewesen ist. Von wenigen Ausnahmen abgesehen, bewegt er sich nur in einem bestimmten lautlichen Bereich - nämlich dem der beiden genannten Laute.

TRASK (2002: 290) verfolgt noch einen weiteren Ansatz, der hier hinsichtlich des Livischen besonders interessant ist. Er beschreibt im Lichte der lexikalischen Diffusion das Auftreten konkurrierender Lautwandel:

,$[\ldots]$ there is no reason at all why the innovations that appear in a language $[\ldots]$ should all be mutually compatible: why shouldn't some of them be in conflict with others? If two conflicting changes appear together, it does not seem unreasonable that they should 'compete' for the same words, with some words eventually going one way and some the other [...]" (TRASK 2002: 290)

Betrachtet man nun das Livische genauer, so lässt sich bei der Entwicklung von $*_{o}$ festhalten, dass hier mehrere Entwicklungen ablaufen: a) $*_{o}>u$, b) $*_{o}>\dot{o}$, c) $*_{o}>u o$ sowie d) $*_{o}>\tilde{o}$. Die 
Lautwandel konkurrieren allesamt um *o und sind dabei verschieden dominant. Die Bedingungen für die ersten beiden Entwicklungen sind klar definierbar: a) tritt vor $m$ auf (POSTI 1942: 10), b) nur im Ost-Livischen nach $m, p$ und $v$ (ebd. 129). Der dritte Lautwandel ist derjenige, der sich im Livischen meist durchsetzen konnte und aufgrund seiner hohen Ausdehnung wohl als älter angesetzt werden kann, sodass d) nur noch wenige Wörter erfasst hat. Dieser Ansatz kann zwar nicht für jeden Beleg begründen, warum der eine Lautwandel dem anderen vorgezogen wurde, er liefert jedoch eine Erklärung dafür, warum derartige Unterschiede vorliegen.

Statistisch gesehen fällt auf, dass Wörter mit einer hintervokalischen zweiten Silbe besonders anfällig für die Lautwandel zu $\tilde{o}$ sind. Dies hat verschiedene Gründe. Hintervokale haben die Lautwandel $* e>\tilde{o}$ und $* e e>\tilde{o} \tilde{o}$ sowie $* e i>\tilde{o} i$ regelmäßig ausgelöst. Auch im Hinblick auf $*_{o}$ spielt der Hintervokal $a$ eine wichtige Rolle. Zwar hat HoLST (2001: 81) die Behauptung aufgestellt, dass dieser Vokal in der zweiten Silbe für den Lautwandel $*_{o}>\tilde{o}$ nicht wesentlich sein könne, seine Vermutung hat sich im Laufe der Analyse jedoch als falsch herausgestellt. Betrachtet man ausschließlich die statistischen Daten, so sticht die hohe Vertretung von hintervokalischen Lauten in dieser Position ins Auge. Von 71 Belegen, die beispielsweise im Estnischen dem Lautwandel $*_{o}>\tilde{o}$ unterliegen, entfallen 17 auf einen Hintervokal in der zweiten Silbe. Davon wiederum ist der Hintervokal in 16 dieser Belege $a$. Das $a$ an dieser Stelle als nicht ausschlaggebend einzustufen, ist von HOLST eine gewagte These. Das Livische kann aufgrund seiner Sonderentwicklung hier nicht berücksichtigt werden, aber ähnliche Verhältnisse wie im Estnischen finden sich auch im Wotischen und Südestnischen. Sie stützen die These, dass das $a$ in der Folgesilbe ein relevanter Laut ist: von insgesamt 67 Belegen, die bei der Entwicklung $*_{o}>\tilde{o}$ einschlägig sind, entfallen im Wotischen allein auf $a$ in der zweiten Silbe 27. Im Südestnischen sind es immerhin noch 11 von 42.

Der Vokal $a$ macht auch unabhängig von der ersten Silbe die häufigste Vertretung in der zweiten Silbe bei einschlägigen Belegen aus: Im Estnischen gehen 83 der insgesamt 185 Entsprechungen des Korpus auf ihn zurück, also fast die Hälfte der Belege. Im Wotischen sind es 82 von 147, im Livischen 56 von 85, im Südestnischen 61 von 144. Auf ein ursprüngliches $a$ in der zweiten Silbe entfällt also in diesen Sprachen immer gut die Hälfte aller Belege. Auf Grundlage der statistischen Daten ist anzunehmen, dass $* a$ für die Herausbildung von $\tilde{o}$ mitverantwortlich ist, vgl. auch Kapitel 6.3. 
Nach der Analyse stellt sich die Frage nach dem Alter der Lautwandel. Eine genaue Datierung ist aufgrund der Beweislage mitunter schwierig, es lassen sich jedoch einige Fakten zum Ablauf einzelner Entwicklungen festhalten und sie so in einen größeren ostseefinnischen Kontext einordnen. Daraus und unter Berücksichtigung weiterer Faktoren ergibt sich abschließend ein ungefähr einzuordnendes Alter der Lautwandel.

Viele der Entwicklungen in den ostseefinnischen Sprachen liegen in mehreren Sprachen vor. Dies bedeutet jedoch nicht zwangsläufig, dass sie alle alt sind oder zumindest ein ähnliches Alter haben: LAANEST (1982: 120) geht beispielsweise von der Möglichkeit einer Parallelentwicklung einzelner Veränderungen in den unterschiedlichen Sprachen aus. Diese Möglichkeit erschwert die eindeutige Klärung der Abfolge einiger Lautwandel, sodass eine Datierung oftmals offenbleibt. Die Analyse der Belege konnte trotzdem einige Hinweise darauf geben, welche Abläufe für die Herausbildung der heutigen Wortformen wichtig sind und in welcher Reihenfolge sie womöglich stattgefunden haben. Diese Erkenntnisse werden im Folgenden in eine chronologische Entwicklung gebracht und zusammengefasst. Dabei werden auch die vor dem Korpus beschriebenen Lautwandel berücksichtigt, sofern sie für die erste Silbe wesentlich sind. Auf eine detaillierte Beschreibung der Lautwandel im Konsonantismus, wie sie VIITSO (1978b) vornimmt, wird an dieser Stelle verzichtet. Stattdessen werden hier die Zeitpunkte einzelner Ableitungen berücksichtigt, da diese zur Klärung einiger Beispiele beitragen.

Eine gesicherte Einordnung ist nicht bei allen Entwicklungen möglich, sodass am Ende eine größere Gruppierung steht, deren Lautwandel anhand der vorliegenden Belege nicht datiert werden können. Innerhalb der einzelnen Kapitel werden die Entwicklungen nochmals unterteilt: Zuerst werden die Lautwandel behandelt, die in allen vier Sprachen vonstattengegangen sind, dann diejenigen, die nur in drei oder zwei Sprachen vorliegen, ehe abschließend auf die Entwicklungen der Einzelsprachen in bewährter Reihenfolge eingegangen wird.

\subsubsection{Vor der Entstehung von $\tilde{o}$ ansetzbare Entwicklungen}

Datierbar auf eine Entstehung vor dem hier untersuchten Lautwandel sind zwar Entwicklungen in allen drei Sprachen, insbesondere jedoch im Livischen. Im Estnischen und Wotischen gibt es nur sehr wenige Veränderungen, die aufgrund der Analyseergebnisse überhaupt als gesichert für diesen Zeitpunkt angesetzt werden können. 


\title{
ENTWICKLUNGEN IN ALLEN SPRACHEN
}

\section{Ableitungssuffix est. -me- / wot. -me- / liv. -m- / estS. -m(e)-}

Vgl. Beleg (175). Ein Suffix mit dem Bestandteil - $m$ - liegt vor. Es sei LAANEST (1982: 206) zufolge alt, da das $-m$ - in allen uralischen Sprachen bekannt sei. Daher ist anzunehmen, dass es wohl bereits vor der Entstehung von $\tilde{o}$ an den Stamm getreten ist. Das heute im Estnischen und Livischen geschwundene $a$ blieb dabei zunächst bewahrt.

\section{ENTWICKLUNGEN IM LIVISCHEN UND SÜDESTNISCHEN}

\section{$* o>\boldsymbol{u}$ vor $\boldsymbol{m}$}

Die Entwicklung $*_{o}>u$ vor $m$ (POSTI 1942: 13; KEEM 2002: 33) verhinderte $*_{o}>\tilde{o}$, z.B. (83) liv. umblõ 'nähen' estS. .umblõma id.

\section{ENTWICKLUNGEN IM ESTNISCHEN}

\section{$* o>e$ in zweiter Silbe}

In Beleg (61) est. nõges 'Nessel', auch est. nõgene, erfolgte zunächst eine Ableitung durch das Suffix - ne, woraufhin sich der Laut der zweiten Silbe hier an die darauffolgende mit $e$ angenähert hat. Es ist davon auszugehen, dass das neue $e$ der zweiten Silbe später den Lautwandel auslöste und somit älter sein muss als $\tilde{o}$. Der Beleg ist in diesem Korpus ein Einzelfall.

\begin{abstract}
Ableitung -ne
Aus der vorausgegangenen Erklärung resultiert auch, dass die Ableitung -ne wohl als älter als die Entstehung von $\tilde{o}$ ansetzbar ist. Das $e$ des Suffixes muss bereits vorgelegen haben, um eine Entwicklung $*_{o}>e$ in nichterster Silbe auslösen zu können.
\end{abstract}

\section{ENTWICKLUNGEN IM WOTISCHEN}

$*_{e}>i$ bzw. $*_{e e}>i i$

Karelischer Einfluss wird bei Belegen wie (60) und (171) angesetzt (VKÄ 126, 131). Dieser muss bereits so früh vorgelegen haben, dass die Lautwandel zu $\tilde{o}$ bzw. õ verhindert wurden. 


\section{ENTWICKLUNGEN IM LIVISCHEN}

\section{Lautwandel von *o und *oi in der ersten Silbe}

$$
*_{o}>\text { uo bzw. *oi > ùoi }{ }^{u} \text { ò̀ }
$$

In der ersten Silbe liegen die Entwicklungen $* o>$ u bei den Monophthongen (PoSTI 1942: 6ff.) und beim Diphthong *oi die Vertrung ùoi $\sim{ }^{u}$ ò̀, im Anlaut auch vùoi $\sim v^{u} o \grave{~}$ (West) oder vọi voì (Ost) (ebd. 31f.) vor, vgl. u.a. die Belege (28) und (188).

$*_{o}>\dot{o}$ nach $m, p$ und $v$ (Ost-Livisch)

Im Ost-Livischen tritt nach den labialen Lauten $m, p$ und $v$ ein Vokal $\dot{o}$ (bei PosTI $g$ ) auf (PosTI 1942: 10), wie u.a. Beleg (85) zeigt.

\section{Epenthese von $u$ der zweiten Silbe}

Die Entwicklung von ${ }^{*} o>$ u sei durch das epenthetische $u$ der zweiten Silbe verhindert worden (POSTI 1942: 7f.), sodass jene wohl ebenfalls älter sein muss als die Entstehung des neuen Lautes, wenn der Lautwandel $*^{o}>$ uo für älter als $\tilde{o}$ angesetzt wird, vgl. livO. z.B. sǒugdâ 'blind' $(<* \operatorname{sog} u \delta a<*$ soke $\delta a)$ (ebd.).

\section{ENTWICKLUNGEN IM SÜDESTNISCHEN}

$* o>\boldsymbol{u}$ vor $h$

Vgl. Beleg (13). Die Entwicklung kann wohl auch als älter angesehen werden, da sie den Lautwandel $* o>\tilde{o}$ verhindert haben muss.

\subsubsection{Nach der Entstehung von $\tilde{o}$ ansetzbare Entwicklungen}

Die Anzahl der Entwicklungen, die für nach der Entstehung von $\tilde{o}$ ansetzbar sind, ist umfangreicher und vielfältiger als die derjenigen, die vor der Herausbildung des neuen Lautes nachweislich abgelaufen sind.

\section{ENTWICKLUNGEN IN ALLEN SPRACHEN}

\section{Ableitungssuffix est. le- $\sim$ wot. -ele-/-ele- $\sim$ liv. -lõ $\sim$ estS. le bzw. lo}

Das Suffix gilt als alt (vgl. LAANEST 1982: 274); für die Wortgruppe von Beleg (55) wird eine germanische Entlehnung erwogen, vgl. urgerm. *metō(ja)n-, *metan- (LÄGLOS II: 281), so- 
dass es erst nach Eintritt des Lehnwortes in die ostseefinnischen Sprachen an den Stamm getreten sein kann. Die Annahme kann dadurch gestützt werden, dass es zumindest im Estnischen noch produktiv ist (KASIK 2015: 160f.). Über die Produktivität in den anderen drei Sprachen liegen keine Daten vor.

\section{ENTWICKLUNGEN IM ESTNISCHEN, LIVISCHEN UND SÜDESTNISCHEN}

\section{Synkope $^{87}$}

Fälle von Synkope treten insbesondere im Livischen und Südestnischen häufig auf. An einigen Stellen ist dieses Phänomen auch für den Lautwandel ausschlaggebend, sodass daraus geschlossen werden kann, dass sie erst nach der Entstehung von $\tilde{o}$ eingetreten ist. Die Laute, die in der zweiten Silbe entfallen sind, müssen beim Aufkommen des neuen Lautes noch vorhanden gewesen sein, da sie oftmals die Entwicklung ausgelöst haben, z.B. in der livischen Entsprechung von (1).

Auch im Estnischen tritt die Synkope bei einigen Belegen auf, wenngleich nicht in ähnlich großer Zahl wie in den anderen beiden Sprachen. Vgl. hier die Ableitung -me in Beleg (175) mit später auftretender Synkope (s.o.).

\section{Apokope}

Der Schwund des vokalischen Auslauts kann ebenfalls erst nach dem Ablauf der Lautwandel eingetreten sein, da der Vokal der zweiten Silbe maßgeblich als Auslöser der Entwicklung fungiert hat. Einige Belege, die diese Annahme stützen, sind im Estnischen und Südestnischen u.a. (53) und (85) sowie im Livischen z.B. (108) und (154).

\section{ENTWICKLUNGEN IM WOTISCHEN, LIVISCHEN UND SÜDESTNISCHEN}

\section{$\tilde{o}$ in nichtersten Silben}

Der Lautwandel in der zweiten Silbe muss ohne Frage ebenfalls sekundär sein, wie bereits eingangs erläutert (vgl. Kapitel 1.3.1). Als Belege für das $\tilde{o}$ in nichterster Silbe können u.a. für das Wotische und Südestnische (1) sowie für das Livische (98) exemplarisch angeführt werden.

\footnotetext{
${ }^{87}$ In Beleg (14) liegt auch im Wotischen eine Synkope vor. Als Einzelfall soll diese hier jedoch nicht näher behandelt werden.
} 


\section{ENTWICKLUNGEN IM ESTNISCHEN}

\section{$* e>\tilde{\boldsymbol{o}}>\boldsymbol{o}$}

Die Entwicklung $* e>\tilde{o}>o$ im Standardestnischen beinhaltet, dass das $\tilde{o}$ in der ersten Silbe entstanden sein muss, bevor es sich in einigen Belegen sekundär zu $o$ weiterentwickeln konnte, vgl. die Belege (16) und (33). Dies deckt sich auch mit den Annahmen VIITSOS (1978b: 94), der den Ablauf für die letzte Stufe seiner Ausführungen annimmt. Es wirkt plausibel, dass diese Verkettung von Lautwandeln später vollzogen wurde, da sich $\tilde{o}$ erst ausgebreitet haben musste.

\section{Abbau der Vokalharmonie}

Die Vokalharmonie kann im Estnischen erst nach dem Aufkommen des neuen Lautes abgebaut worden sein. Davon zeugt auch der heutige Wortschatz. Es finden sich viele Wörter, in denen das * $e$ der ersten Silbe bewahrt wurde, die Bedingungen in der heutigen Wortform erfüllt werden: z.B. est. tera 'Schneide, Klinge' ( fi. terä 'Klinge, Schneide, Sägeblatt, Bohrer') oder est. elada 'leben' ( fi. elää id.). Der Lautwandel erfolgt nachträglich nicht mehr.

\section{ENTWICKLUNGEN IM LIVISCHEN}

\section{$-i$ im Wortauslaut}

Im Falle des livischen Belegs (67) liegt im Auslaut $-i$ vor. Da als rekonstruierter Wortstamm urfi. *hepo angesetzt wird (LÄGLOS I: 95), ist von einer späteren Entstehung des Vordervokals in wortletzter Silbe im Livischen auszugehen, vielleicht als analoge Form zu Wörtern wie liv. pu'nni 'rot' ( fi. punainen id.) oder liv. u'ddi 'nebelig' (<*utuinen). Dort sei der erste Diphthongbestandteil geschwunden, wenn als zweiter $i$ vorlag (LAANEST 1982: 131).

\subsubsection{Entwicklungen, die nicht datiert werden können}

Zu den Verhältnissen, die deutlich eingeordnet werden können, kommen einige Entwicklungen, die zwar für die erste Silbe relevant sind, jedoch aus unterschiedlichen Gründen nicht genauer datiert werden können. Sie helfen also nur bedingt, die Lautwandel in der lautlichen Entwicklung insgesamt zu verorten. 


\section{ENTWICKLUNGEN IM ESTNISCHEN, WOTISCHEN UND LIVISCHEN}

$*_{n s}>V s$

In den drei genannten Sprachen gibt es die Vokalisierung des Nasals $*_{n} n$ in Verbindung mit dem Sibilanten $s$ (also *ns $>V s$ ), vgl. die Belege (86) und (106). Dass die Vokalisierung auch flächendeckend abgelaufen ist, spricht dafür, dass sie noch aus einer gemeinsamen Sprachform stammen könnte (vgl. auch LAANEST 1982: 124), wenngleich nicht eindeutig ist, ob die Entwicklung tatsächlich vor der Entstehung von $\tilde{o}$ eingetreten oder eine parallele Entwicklung der drei Sprachen sein kann, wie sie LAANEST (ebd. 120) für einige (von ihm nicht näher bestimmte) Lautwandel vermutet. Gegen eine frühe Vokalisierung spricht der Lautwandel von * $o$. Wie sich in der Analyse gezeigt hat, unterliegt in langer Vertretung nur *ee dem Lautwandel zu õõ. Hätte es die Vokalisierung von $* n$ schon früh gegeben, wäre das Resultat ein Lautwandel $* o o>\tilde{o} \tilde{o}$ gewesen, auf den es keinerlei Hinweise gibt. Der genaue Zeitpunkt der Vokalisierung bleibt ungewiss. Auch VIITSO (1978b: 93, 102, 104) ist in seinen Angaben wenig aufschlussreich, da er zwar Entwicklungen zum Lautwandel $* V n s>V V s$ und seiner Herausbildung in den einzelnen Sprachen anführt, ihn aber lediglich in das gleiche Zeitfenster einordnet wie das Auftreten von $\tilde{o}$.

\section{ENTWICKLUNGEN IM ESTNISCHEN, WOTISCHEN UND SÜDESTNISCHEN}

$u \sim v$

$\mathrm{Zu}$ welchem Zeitpunkt die Entsprechungen von $u \sim v$ aufgekommen sind, ist ebenso wenig datierbar. Belege sind u.a. (54), (178) und (197).

\section{Verbalableitung $-u$ -}

Unklar ist ebenso, wann die Verbalableitung $u$ an die betroffenen Wortstämme getreten ist. Viele von ihnen lassen sich auf keinen Urstamm zurückführen, sodass eine Bewertung des zeitlichen Ablaufs erschwert wird. Belege dafür sind u.a. (46), (136) oder (184).

\section{ENTWICKLUNGEN IM ESTNISCHEN UND WOTISCHEN}

\section{Nominalableitung $-u$}

Eine Ableitung, die nicht datiert werden kann, ist $u$, wie es in Beleg (167) im Estnischen oder (125) im Wotischen auftritt. 


\section{Verbalableitung - $i$ -}

Ebenfalls unbestimmbar ist der Zeitpunkt der Verbalableitung $i$. Sie führt wie in Beleg (139) zu unterschiedlichen Verhältnissen in den einzelnen Sprachen. Es kann nicht bestimmt werden, ob zuerst die Ableitung eingetreten ist, wie es zur wotischen Entsprechung passen würde, oder erst der Lautwandel, wie das estnische Wort erwarten lässt, wenn man von einer ursprünglich hintervokalischen zweiten Silbe ausgeht - oder gar, ob man es in den Einzelsprachen mit verschiedenen zeitlichen Abläufen zu tun hat.

\section{ENTWICKLUNGEN IM ESTNISCHEN UND SÜDESTNISCHEN}

\section{Metathese *ouh > ohv}

Die Metathese *ouh > ohv tritt in Beleg (178) auf. Zwei Abläufe sind möglich: Entweder, der Lautwandel zu $\tilde{o}$ trat zuerst ein und bewirkte eine lautgesetzliche Entwicklung *ou > $\tilde{\text { ou, oder, }}$ die Metathese erfolgte zuerst und der Vordervokal der zweiten Silbe bewirkte später ganz regelmäßig einen Lautwandel $* o>\tilde{o}$.

\section{Ableitung -ke(ne)}

Eine weitere Ableitung, deren Zeitpunkt des Aufkommens in diesem Kontext nicht ermittelt werden kann, ist -ke(ne), vgl. Beleg (75). Sie gilt im Standardestnischen noch als produktiv (KASIK 2015: 251), was die Datierung nicht vereinfacht. Für das Südestnische liegen keine Daten dazu vor.

\section{$* o>u$ in nichtersten Wortsilben}

Die Entwicklung $*_{o}>u$ weiter hinten als in der ersten Wortsilbe liegt im Estnischen und Südestnischen flächendeckend vor und spricht somit für ein gewisses Alter, jedoch ist anhand der Belege nicht erkennbar, ob dieser Lautwandel vor oder nach der Entstehung von $\tilde{o}$ abgelaufen ist. Aufgrund der hintervokalischen Qualität beider Laute ist dieser Lautwandel nicht von entscheidender Wichtigkeit für die Entwicklung der ersten Silbe. Belege aus dem Korpus sind u.a. (157) und (181). 


\section{ENTWICKLUNGEN IM WOTISCHEN UND SÜDESTNISCHEN}

\section{Ableitung -o}

Das Alter der Ableitung, wie sie in Beleg (160) auftritt, kann nicht datiert werden, da auch im Wortstamm von einem Hintervokal ausgegangen werden kann und nicht ersichtlich ist, welcher der beiden Vokale den Lautwandel in der ersten Silbe ausgelöst hat.

\section{ENTWICKLUNGEN IM ESTNISCHEN}

\section{$* k l>V l$}

In einigen Belegen wird der Klusil $k$ im Silbenauslaut im Estnischen vor $l$ vokalisiert (EKÄ 86), vgl. (60) und (122). Ob diese Entwicklung noch vor dem Aufkommen des neuen Lautes $\tilde{o}$ eingetreten ist, kann aufgrund der unzureichenden Beleglage nicht näher bestimmt werden.

\section{Ableitung - $e$}

Aufgrund fehlender Daten kann der genaue Eintritt des Suffixes - $e$ im Estnischen nicht sicher bestimmt werden, vgl. Beleg (186) est. kõu, gen. - $e$ 'Donner'.

\section{ENTWICKLUNGEN IM LIVISCHEN}

\section{$*_{i}$ als zweiter Diphthongbestandteil $>\boldsymbol{u}$ vor $v$}

Wann $i$ als zweiter Bestandteil des Diphthongs vor $v$ zu $u$ wird, konnte aufgrund der geringen Anzahl einschlägiger Belege nicht ermittelt werden. Im Korpus existiert lediglich (96). Beide Entwicklungen, $* o i>\tilde{o} i>\tilde{o} u$ oder $* o i>* o u>\tilde{o u}$, scheinen möglich.

\section{$* \ddot{a}>a$ in nichtersten Silben (Abbau der Vokalharmonie)}

Posti (1942: 42) setzt den Lautwandel $* a>\ddot{a}$ in nichtersten Silben im Livischen als früh an und die hohe Ausbreitung spricht für ein gewisses Alter. Der oben ausführlich diskutierte Beleg (56) wirft Fragen zur zeitlichen Einordnung auf. VIITSO (1978b: 95) erwägt die Entwicklung * $\ddot{a}$ $>a$ im Livischen als Begleiterscheinung des Lautwandels $* e>\tilde{o}$ und setzt sie somit für den gleichen Zeitraum an. Die abschließende Bewertung bleibt offen. 


\section{ENTWICKLUNGEN IM SÜDESTNISCHEN}

\section{Assimilation des zweiten Diphthongbestandteils an den ersten}

Wann in der ersten Silbe der zweite Diphthongbestandteil im Südestnischen an den ersten assimiliert wurde, kann anhand der vorliegenden Belege wie z.B. (213) nicht sicher bestimmt werden. Eine sekundäre Entwicklung scheint möglich, da hier $* V_{1} V_{2}>\tilde{o} \tilde{o}$ auftritt.

\section{Palatalisation}

Auch für die Palatalisation kann diskutiert werden, ob es eine späte Entwicklung ist, die Belege sind hierbei jedoch nicht eindeutig genug.

\section{$* \tilde{\boldsymbol{\sigma}} \tilde{\boldsymbol{\sigma}} \boldsymbol{\tilde { \boldsymbol { \sigma } } \boldsymbol { i }}$}

Wann der Langvokal der ersten Silbe diphthongiert wurde, kann nicht näher bestimmt werden, da zu wenig Belege davon betroffen sind. Vgl. hier (165).

\subsubsection{Zusammenfassender Überblick über die Lautwandel}

Zusammengefasst lassen sich die im vorherigen Teil des Kapitels beschriebenen Lautwandel in ihrer Abfolge mit Beispielen aus der Diskussion wie in den nachfolgenden Kapiteln tabellarisch darstellen. Auch hier gilt das bekannte Schema bei der Reihenfolge der Darstellung.

\subsubsection{Estnisch}

Die Entwicklungen im Estnischen lassen sich wie nachfolgend zusammenfassen:

Vor Entstehung von $\tilde{o} \quad *_{o}>e$ in zweiter Silbe

(61) est. nõges 'Nessel', auch est. nõgene

Ableitungssuffix -me-

(175) est. sõ̃or|e, gen. -me 'Nasenloch, Nüster'

Ableitung -ne

(61) est. nõges 'Nessel', auch est. nõgene

Nach Entstehung von $\tilde{o} \quad$ Synkope

(175) est. sõorre, gen. -me 'Nasenloch, Nüster' 
Apokope

(53) est. $m \tilde{r} r \mid d$, gen. - $r a$ 'Fischreuse'

$*_{e}>\tilde{o}>o$

(33) estS. kõ |tar', gen. -dara 'Speiche' [estN. kodar, gen. $a$ id.]

Abbau der Vokalharmonie

est. elada 'leben' ( fi. elää id.)

Ableitungssuffix $l e-$

(55) est. mõtelda 'denken, nachdenken'

Nicht datierbar $*_{n s}>V s$

(86) est. $\tilde{o} \tilde{o} \mid s$, gen. -ne 'Höhle, Höhlung'

(106) est. põ̃osas 'Busch'

$u \sim v$

(113) est. põu, gen. - $e$ 'Brust'

$* k l>V l$, wenn $k$ im Silbenauslaut steht

(60) est. nõel, gen. - $a$ 'Nadel'

(122) est. sõel, gen. - $a$ 'Sieb'

Metathese *ouh $>$ ohv

(178) est. jõhv, gen. - $i$ 'Pferdehaar, Rosshaar'

$*_{o}>u$ in nichtersten Wortsilben

(157) est. $v \tilde{o r} \mid k$, gen. - $g u$ 'Netz'

(181) est. jõu|k, gen. -gu 'Bande, Rotte, Horde'

Ableitung $-u$ (nominal)

(169) est. mõ $\mid t$, gen. $-d u$ 'Maß, Ausmessung'

Ableitung $-u$ (verbal)

(46) est. mõjuda 'beeinflussen, wirken'

(136) est. sõtkuda 'treten, kneten'

Ableitung - $i$

(139) est. tohtida 'dürfen'

Ableitung - $e$

(186) est. kõu, gen. - $e$ 'Donner'

Ableitung -ke(ne)

(75) est. ôhuke 'dünn' 
Ableitung -ne

(201) est. oud(ne) 'unheimlich'

\subsubsection{Wotisch}

Die Entwicklungen im Wotischen lassen sich wie folgt zusammenfassen.

Vor Entstehung von $\tilde{o} \quad$ Ableitung -me

(175) wot. sẹrr(a)med 'Nasenloch, Nüster'

$*_{e}>i \mathrm{bzw} .{ }^{*} e e>i i$

(60) wot. nigla 'Nadel'

(171) wot. piina 'Pfosten, Pfeiler'

Nach Entstehung von $\tilde{o} \quad$ Auftreten von $\tilde{o}$ in nichtersten Silben

(1) wot. õpõa 'Silber'

Ableitung -ele-/-ele-

(55) wot. mõtõlla 'denken, nachdenken'

Nicht datierbar

$* n s>V s$

(86) wot. $\tilde{o} \tilde{s} i$, gen. $\tilde{o} \tilde{\text { nõ } \tilde{o} ~ ' H o ̈ h l e, ~ H o ̈ h l u n g ' ; ~}$

(106) wot. põ̃zaz 'Busch'

$u \sim v$

(197) wot. nõvvo 'Rat, Ratschlag'

Nominalableitung $-u$

(125) wot. sõlm $\mid u$, gen. - -иu 'Knoten'

Verbalableitung $-u-$

(136) wot. sõtkoa/-ua 'treten, kneten'

Verbalableitung $-i$ -

(139) wot. tõhtia

Ableitung - $O$

(160) wot. võso 'Schössling, Spross' 


\subsubsection{Livisch}

Im Livischen hat man es zusammengefasst mit den nachfolgenden Entwicklungen zu tun. Vor Entstehung von $\tilde{o} \quad$ In der ersten Silbe:

$*_{o}>$ иo

(28) liv. kuordõ 'hoch'

$*_{o}>u$ vor $m$

(83) liv. umblõ 'nähen'

$*_{o}>\dot{o}$ nach $m, p$ und $v$ (Ost-Livisch)

(85) liv. vòn 'Glück'

*oi > ùoi ${ }^{u} o \grave{l}$

(188) liv. lūoima 'Weberkette'

Epenthese von $u$ der zweiten Silbe

(123) livO. sǒugdâ 'blind'

Ableitung -m-

(175) liv. sõ̃rmõz 'Nasenloch, Nüster'

Nach Entstehung von $\tilde{o}$ Synkope

(1) liv. $\tilde{o}^{\prime} b d \tilde{o}$ 'Silber'

Apokope

(100) liv. $p \overline{\tilde{o}} k t$ 'feiner Staub'

Auftreten von $\tilde{o}$ in nichtersten Silben

(98) liv. põ'ddõrz 'Elch'

$-i$ im Wortauslaut

(67) liv. $\tilde{o}^{\prime} b b i$ 'Pferd'

Ableitung -lo

(55) liv. mõtlõ 'denken, nachdenken'

Nicht datierbar $* n s>V s$

(86) liv. õ̃n 'Höhle, Höhlung';

(106) liv. pȭzõ 'Busch'

$* i$ als zweiter Diphthongbestandteil $>u$ vor $v$

(185) liv. kõuvõ 'Birke'

$* \ddot{a}>a$ in nichtersten Silben (Abbau der Vokalharmonie)

(56) liv. mõts $\bar{a}$ 'Wald' 


\subsubsection{Südestnisch}

Vor Entstehung von $\tilde{o} \quad *_{o}>u$ vor $m$

(83) estS. .umblõma 'nähen'

$*_{o}>u$ vor $h$

(13) estS. kuht, gen. koha kuha 'Ort, Stelle'

Ableitung - $m(e)$ -

(175) estS. sõormas, gen. sõ õrma; sõ̃rmõs, gen.

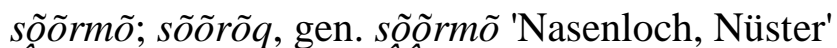

Nach Entstehung von $\tilde{o} \quad$ Ableitung le- bzw. ló-

(55) estS. .mõtlõma, II. Infinitiv mõtõldaq 'denken, nachdenken'

Synkope

(43) estS. .lõpma, II. Infinitiv lõppõq 'beenden' Apokope

(53) estS. mõrd, gen. mõrra 'Fischreuse'

(85) estS. õnn', gen. õnnõ 'Glück'

$\tilde{o}$ in nichtersten Silben

(1) estS. hõpõ 'Silber'

Nicht datierbar $* \tilde{o} \tilde{o}>\tilde{o} i$

(165) estS. lốig, gen. lõia 'Leine'

$u \sim v$

(189) estS. lõug, gen. lõvva 'Kinn'

Metathese *ouh > ohv

(178) estS. jõhv', gen. jõhvi; jõuh, gen. jõuhõ 'Pferde-

haar, Rosshaar'

Ableitung -ke(ne)

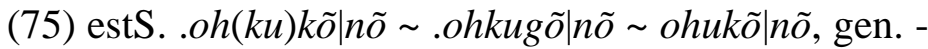
sõ 'dünn'

Assimilation des zweiten Diphthongbestandteils an den ersten

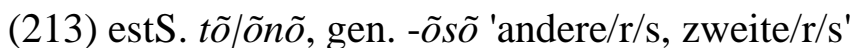


Palatalisation

(99) estS. põhi, gen. põh'a 'Boden, Grund'

$*_{o}>u$ in nichterster Silbe (vgl. KeEM 2002: 34)

(157) estS. võrk, gen. võrgu 'Netz'

Ableitung $-u-$

(184) estS. .kõikma, II. Infinitiv .kõikuq 'sich bewegen, schwanken, schaukeln'

Ableitung - $O$

(160) estS. võso 'Schössling, Spross'

\subsection{Zur Ausbreitung und zum Alter der Lautwandel zu $\tilde{o}$}

Nach der groben zeitlichen Einordnung der Entstehung von $\tilde{o}$ durch die Betrachtung weiterer Lautwandel sowie der Ableitungssuffixe bleibt zu klären, welche Erkenntnisse sich nun über die Ausbreitung und das Alter des neuen Lautes gewinnen lassen.

Dass es sich bei õ um ein Phänomen handelt, das erst in einer späteren ostseefinnischen Sprachstufe aufgekommen ist, lässt sich zunächst dadurch begründen, dass es im Ostseefinnischen anscheinend keine Fortsetzer eines uralischen Lautes von ähnlicher Qualität gibt (vgl. die Diskussion um Sammallahtis Theorie sowie ItKOnEns Ausführungen in Kapitel 2.). Es gibt keine Belege dafür, dass man es hier mit einem Laut aus einer ostseefinnischen Grundsprache zu tun hat, denn bei den hier behandelten Sprachen spricht nichts für einen Abbau von $\tilde{o}$ im Vokalismus der ersten Silbe. VIITSO (1978b: 89) führt ferner an, dass der Laut als einziger nicht im Auslaut von einsilbigen ostseefinnischen Wortstämmen stehe und sieht dies als ein weiteres Argument gegen die Ursprünglichkeit von o. All diese Umstände deuten bei der Entstehung von $\tilde{o}$ bereits auf eine späte Entwicklung hin.

Die Sprachen im Norden und Süden dieses Sprachzweigs müssen sich beim Aufkommen des neuen Lautes $\tilde{o}$ schon in wenigstens zwei Teile aufgespalten haben, ehe er sich in den einzelnen Sprachen verbreitet hat. Eine Aufteilung nimmt SuHONEN (1988: 288) bereits für das Späturfinnische an, sodass es sich beim Entstehungszeitraum des neuen Lautes $\tilde{o}$ bereits um diese oder sogar eine noch spätere Phase handeln muss. Wäre der neue Laut älter gewesen, hätte es wohl auch Hinweise auf ihn in der Nordgruppe gegeben, die Verbreitung hätte gleichmäßiger sein müssen und auch das Livische wäre wohl stärker von den Lautwandeln erfasst worden. Statt- 
dessen hat es eine eigene Entwicklung durchgemacht, die nur aus einer Aufspaltung der Sprachen vor dem Aufkommen des Lautwandels plausibel zu erklären ist. Dass die Verbreitung von $\tilde{o}$ im Estnischen sehr stark ist, im Wotischen und Südestnischen immer noch ähnlich häufig, im Livischen dagegen nicht, ist ein weiteres Indiz, das darauf hindeutet, dass dieser Vokal erst spät entstanden sein kann und eine recht lokale Entwicklung ist. Gestützt werden kann diese Vermutung teilweise auch durch VIITsos Annahmen. Das Aufkommen von õ setzt er auch unter Berücksichtigung weiterer Lautwandel für einen Dialektverbund ${ }^{88}$ an, der aus den ostseefinnischen Sprachen der Südgruppe bestehe (VIITSO 1978b: 92, 104). Eine der Sprachen als konkreten Ausgangspunkt für die Entwicklung nennt er dabei in seinen Ausführungen nicht.

Weitere Hinweise zum Alter von $\tilde{o}$ geben die Lehnwortschichten. Für die ostseefinnischen Sprachen werden gemeinhin vier verschiedene Hauptphasen an Entlehnungen angenommen (SUHONEN 1988: 311):

1. Indoiranische/arische Entlehnungen;

2. Baltische Entlehnungen;

3. Germanische Entlehnungen;

4. Slawische Entlehnungen.

Die Phase der indoiranischen bzw. arischen Entlehnungen ist die älteste in diesem Sprachzweig. Man geht davon aus, dass viele dieser Lehnwörter bereits in die finnougrische Grundsprache eingetreten sind, während weitere sowie die anderen drei Stufen erst für die urfinnische Zeit angesetzt werden können (ebd.). Da die Lehnwörter schon vor einer für die Entstehung von $\tilde{o}$ relevanten Zeit in den ostseefinnischen Sprachen vorgelegen haben. ist die erste Stufe für die Analyse der Lautwandel nicht von größerer Wichtigkeit.

Über das Alter der mittleren beiden Stufen herrscht Uneinigkeit, wohl auch, weil die baltischen und germanischen Lehnwörter teilweise zeitgleich in die ostseefinnischen Sprachen eingetreten sein können. SUHONEN (ebd. 311f.) gibt an, dass die ältesten Lehnwörter der beiden Stufen bereits früh in das Urfinnische gelangt seien, „da Urlappisch und Urfinnisch noch eine relativ einheitliche Gesamtheit bildeten“. LAANEST (1982: 321) nimmt die baltischen Lehnwörter als älteste Schicht an und beruft sich auf Archäologen, die von Kontakten der baltischen und ostseefinnischen Stämme im 2. oder schon am Ende des 3. Jahrtausends v.Chr. ausgehen. Dem

\footnotetext{
${ }^{88}$ Als Dialektverbund definiert VIITSO (1978b: 91) eine Gruppierung aller Dialekte, die einer neuen Entwicklung unterliegen.
} 
pflichtet man in LÄGLOS (I: XVI) bei. Gefolgt werde sie von der germanischen Lehnwortschicht (LAANEST 1982: 327), deren älteste Lehnwörter aus der Mitte des 2. Jahrtausend v.Chr. stammen. Jüngere germanische Entlehnungen setzten um das Jahr 500 n.Chr. ein (LÄGLOS I: XVI). Das Alter der baltischen Entlehnungen begründet LAANEST (1982: 325) mit einer gleichmäßigen Verbreitung der Lehnwörter und den ostseefinnischen Lautwandeln, die sie durchlaufen haben müssen, z.B. * $t i>s i$. Die Verbreitung der germanischen Lehnwörter sei dagegen weit differenzierter, denn es gebe Beispiele, die nur in die Nordgruppe der ostseefinnischen Sprachen entlehnt wurden, in der Südgruppe aber nicht auftreten (ebd. 331). ${ }^{89}$ Den Lautwandel $* t i>s i$ setzt SUHONEN (1988: 312) auch bei germanischen Entlehnungen an.

Einig ist man sich bei den slawischen Lehnwörtern: Ihre Anfänge können auf das 1. Jahrtausend n.Chr. datiert werden (LAANEST 1982: 338; SUHONEN 1988: 312; LÄGLOS I: XVI). ${ }^{90} 91$

Viele der vom Lautwandel betroffenen Belege des hier untersuchten Koprus lassen sich als baltische oder germanische Lehnwörter identifizieren (63 der insgesamt 226 Belege). Davon sind 35 germanischen Ursprungs (21 gesichert, 14 weitere erwogen), auf die baltischen Sprachen gehen 23 zurück (17 gesichert, sechs erwogen). Bei fünf Belegen ist unsicher, ob es sich um baltische oder germanische Lehnwörter handelt. Die Lehnwörter aus diesen beiden Schichten machen zusammengenommen also mehr als ein Viertel der Belege aus (ca. 27,88\%). ${ }^{92}$

Die baltischen und germanischen Lehnwörter müssen bereits vorhanden gewesen sein als $\tilde{o}$ aufkam, da sie ebenfalls weitreichend und recht regelmäßig von diesen Entwicklungen erfasst wurden. Es lässt sich also festhalten, dass $\tilde{o}$ jüngeren Alters sein muss als der Eintritt der baltischen und germanischen Lehnwörter ins Ostseefinnische. Ansetzbar ist auch, dass er vor einem verstärkten Eintritt der slawischen Lehnwörter aufgekommen ist, denn im untersuchten Korpus treten diese so gut wie nicht auf, sind also kaum vom Lautwandel zu o betroffen. Lediglich der

\footnotetext{
${ }^{89}$ Als Beispiele für Entlehnungen, die nur in der Nordgruppe der ostseefinnischen Sprachen vorkommen, nennt LAANEST (1982: 331) an dieser Stelle u.a. fi. aalto 'Welle' kar. (Olonetz) aldo id. weps ald id. oder fi. paita 'Hemd' kar. paida id. weps. paid' id.

${ }^{90} \mathrm{Zu}$ den ersten Kontakten des Estnischen mit dem Slawischen vgl. auch BLOKLAND (2009: 17ff.).

${ }^{91}$ VIITSO (1998a: 113f.) differenziert die einzelnen Lehnwortschichten ähnlich, wenngleich er keine Daten nennt. Neben den bereits genannten Entlehnungen nennt er jene aus den Kontaktsprachen, wie dem Schwedischen, das v.a. für das Finnische und teilweise das Estnische relevant war. Für das Estnische und Livische kommen noch hoch- und niederdeutsche Entlehnungen infrage, außerdem v.a. für das Livische lettische Entlehnungen. Russische Entlehnungen setzt er vorwiegend für das Ingrische, Karelische, Wepsische und Wotische an, während die anderen Sprachen weniger stark davon beeinflusst wurden. Insbesondere die Entlehnungen aus den Kontaktsprachen sind für die Lautwandel nicht von größerer Bedeutung, da sie jünger sein müssen als $\tilde{o}$.

92 Weitere 26 Belege dieses Korpus (ca. 11,5\%) werden als deskriptiv oder onomatopoetisch bezeichnet. Hinzu kommen vereinzelte Lehnwörter anderer Schichten, z.B. indoiranische oder auch jüngere, wie slawische oder skandinavische. Sie liegen jedoch nur in geringer Anzahl vor.
} 
Beleg (154) est. võlu 'Zauber' liv. võ'l 'Zauberer, Hexe' (< russ. volhv 'Wahrsager, Zauberer/Hexe, Seher' [...] (SSA III: 423)) kann hier den slawischen Entlehnungen zugeschrieben werden.

Wo der Ursprung des neuen Lautes liegt, ist bestimmbar. Forscher verschiedener Positionen (vgl. z.B. HAKUlinen (1979) und Kallio (2014); vgl. auch Kapitel 2) haben ihn u.a. in den germanischen Lehnwörtern gesucht, da diese häufig $a$-stämmig waren. ${ }^{93}$ Bereits VIITSO (1978b: 89) stellte fest, dass es sich bei den Wörtern, die einen Lautwandel zu $\tilde{o}$ vollziehen, zu einem großen Teil um Entlehnungen aus dem Germanischen oder Baltischen handelt. Die Resultate der vorliegenden Untersuchung stützen diese Annahme, wie die oben dargelegten Verhältnisse der Entlehnungen zeigen. Dass es frühe Entlehnungen sind und die Wörter häufig keine Entsprechungen außerhalb der ostseefinnischen Sprachen haben, ist für VIITSO (ebd.) ein Argument gegen eine Annahme eines primären o. Die Belege, die eine Entsprechung in anderen finnougrischen Sprachen besitzen, geben ihm zufolge ebenfalls keinen Hinweis auf ein altes $\tilde{o}$. Diese Theorien lassen sich aufgrund des vorliegenden Korpus bekräftigen. Viele der Wortstämme mit einschlägigem Lautwandel gehen auf Entlehnungen zurück; nur ein Bruchteil der Belege ist überhaupt über das Ostseefinnische hinaus etymologisch gesichert. ${ }^{94}$ Die Anzahl jener, bei denen die etymologischen Wörterbücher eine gesicherte uralische Form ansetzen, beträgt lediglich vier, im Falle der finnougrischen Wörter sind es 13 der Belege. Zusammengenommen machen diese 17 Belege lediglich ca. 7,52\% des gesamten Korpus aus.

Aufgrund der hohen Regelmäßigkeit in allen vier Sprachen ist anzunehmen, dass der Ausgangspunkt des Lautwandels in der ersten Silbe wohl das $* e$ sein muss. Die Entwicklungen von $* o$ sind zu ungleichmäßig und unterschiedlich, als dass sie als Ausgangspunkt für den Lautwandel realistisch erscheinen. Insofern lässt sich ITKONENS (1945: 178f.) Annahme einer Bildung von $\tilde{o}$ aus einem leicht velarisierten $e$ heraus durch die hiesige Betrachtung stützen. Ferner beschreibt er, dass in den Wörtern, in denen das * $e$ in der ersten Silbe erhalten bleibt, in der Folgesilbe immer ein ursprünglich vordervokalischer Stamm vorliegt (ebd. 170), was für ihn ein Beweis eines sekundären $\tilde{o}$ ist. Seine Annahme deckt sich auch mit den Ergebnissen dieser

\footnotetext{
${ }^{93}$ Einen Laut $\tilde{o}$ kennen die germanischen und baltischen Sprachen selbst nicht, sodass er keine Entlehnung sein kann, vgl. für das Germanische LÄGLOS (I: X) und für die baltischen Sprachen DINI (2014: 102ff.). Man hat es also mit einer Innovation aus den ostseefinnischen Sprachen zu tun.

${ }^{94}$ Dies ist keine neue Erkenntnis: SUHONEN (1988: 311) schreibt beispielsweise, dass mit Ausnahme der deskriptiven Wörter nur von einer kleinen Menge an „Urschöpfung am ostseefi. Wortschatz“ auszugehen ist.
} 
Untersuchung. Von den wenigen Abweichungen im Livischen bzw. Südestnischen abgesehen hat die Analyse keine ursprünglich vordervokalischen Stämme ergeben, die einen Lautwandel $*_{e}>\tilde{o}$ durchlaufen haben. VIITSO (1978b: 92) ist sich ebenfalls sicher, dass es zunächst einen Lautwandel $* e>\tilde{o}$ bei nicht abgeleiteten Wörtern mit hintervokalischen Wurzeln gegeben habe. Ein Lautwandel $*_{o}>\tilde{o}$ sei dann seiner Auffassung nach zunächst in etwa zehn Stämmen auf-

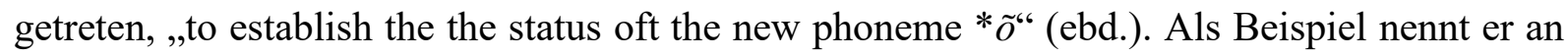
dieser Stelle u.a. *votta- > võtta- 'nehmen'. Warum er sich auf lediglich etwa 10 Stämme beschränkt, scheint in seinen Ausführungen zunächst nicht ersichtlich, deutet jedoch auf einen Prozess hin, denn weitere Lautwandel $*^{*}>\tilde{o}$ setzt er für spätere Entwicklungsstufen an, z.B. für jene nach Auflösung der Dialektverbünde (ebd. 94f.).

Im Hinblick auf die Langvokale lässt sich ohnehin nur ein einziger Lautwandel festhalten, *ee > $\tilde{o}$. Dass der Lautwandel hier bei Langvokalen mit * $e$ als Qualität vorliegt, scheint ITKONENS Annahme zu bekräftigen und ist ein weiteres Anzeichen dafür, dass der Laut $* e$ das Zentrum der Lautwandel gewesen sein muss. Wäre es $* o$, so wäre wohl auch eine Entwicklung *oo > $\tilde{o} \tilde{o}$ zu erwarten gewesen, für welche es jedoch keinerlei Anzeichen gibt.

Das Bild der Diphthonge ist im Gegensatz zu den anderen Kategorien weit ambivalenter. Sie teilen sich in zwei Gruppen auf: Einerseits gibt es diejenigen, in denen die Lautwandel regelmäßig sind und es im alten Wortschatz mehr oder weniger keine Ausnahmen gibt (*eu und *ou sowie *ei). Andererseits existieren jene, in denen es deutliche Abweichungen gibt (*oi). Warum die Lautwandel $* e u>\tilde{o u}$ und $* o u>\tilde{o u}$ lautgesetzlich sind, *ei > $\tilde{o} i$ sich sehr regelmäßig verhält, *oi in seiner Entwicklung jedoch eine wechselnde Vertretung hat, lässt Raum für Interpretation. Schon bei den Kurzvokalen konnte festgestellt werden, dass im Rahmen des Lautwandels $*_{o}>$ $\tilde{o}$ häufiger Abweichungen auftreten und eine Zweiteilung vorliegt.

Auch die Zweiteilung der Diphthonge mit *o als erstem Bestandteil deutet darauf hin, dass der Lautwandel nicht von dort ausgegangen sein kann. Zwar ist die Vertretung der einschlägigen Belege mit $* e$ als erstem Bestandteil gering, jedoch ist sie im Gegensatz zu der von $* o$ regelmäßig. Dass der Diphthong*ei im Livischen nicht vom Lautwandel zu õi erfasst wurde, könnte ein weiteres Zeichen für das spätere Aufkommen von $\tilde{o}$ in dieser Sprache sein.

Ein Kontinuum in der Form, wie es aufgrund des Lautwandels $*_{o}>\tilde{o}$ von KALLIO (2014: 161) und KETTUNEN (EKÄ 132) angenommen wird, lässt sich anhand der Belege dieser Untersuchung kaum beweisen. KALLIO (2014: 161), der bei $* o>\tilde{o}$ von einer ,,sporadischen Illabialisierung“" ausgeht, ist beispielsweise der Ansicht, dass die Verbreitung dieses Lautwandels vom 
Wotischen über das Nordestnische, dann Südestnische bis hin zum Livischen abgenommen hat. Dies wirkt vor dem Hintergrund der hier diskutierten Belege unwahrscheinlich. Betrachtet man die reine Datenmenge, so wird deutlich, dass das (Nord-)Estnische in der Gesamtzahl der Belege, die dem Lautwandel unterliegen, stärker vertreten ist als das Wotische (Estnisch $185 \mathrm{Be}$ lege vs. Wotisch 147 Belege). Das Südestnische macht mit 144 Belegen in der Gesamtzahl kaum einen Unterschied zum Wotischen aus. Auch ist die Anzahl der einschlägigen Belege, die auf *o zurückgeführt werden können, im Estnischen im Vergleich zum Wotischen knapp etwas höher (hier Estnisch: 71 Belege; Wotisch: 67 Belege). Die Annahme KALLIOS, dass der neue Laut $\tilde{o}$ im Wotischen bei Belegen des Lautwandels $* o>\tilde{o}$ am häufigsten vorliegt und durch die Dialekte bis hin zum Livischen abnimmt, deckt sich also nicht mit allen Ergebnissen dieser Arbeit. Dass das Livische die Entwicklungen weit weniger vollzogen hat, kann bestätigt werden.

Vor dem Hintergrund der verschiedenartigen Ausbreitung des Lautes $\tilde{o}$ kann wohl von einer Entwicklung ausgegangen werden, wie sie von JOHANNES SCHMIDT (1872) bereits im 19. Jahrhundert beschrieben wird. Er postuliert anhand der indogermanischen Sprachen und deren Entwicklung die sogenannte Wellentheorie:

„Ich möchte an seine [des Stammbaums] stelle das bild der welle setzen, welche sich in concentrischen mit der entfernung vom mittelpunkte immer schwächer werdenden ringen ausbreitet. Dass unser sprachgebiet keinen kreis bildet, sondern höchstens einen kreissector, dass die ursprünglichste sprache nicht im mittelpunkte, sondern dem einen ende des gebietes ligt, tut nichts zur sache." (SCHMIDT 1872: 27)

Laut dieser Theorie gehen Entwicklungen von einer Sprache oder einem Sprachgebiet aus und werden schwächer, je weiter sie sich davon entfernen. SCHMIDT (ebd. 15f.) notiert, dass Sprachen, die in unmittelbarer geographischer Nähe zueinander vorkommen, mehr Gemeinsamkeiten vorweisen als jene, deren Sprachgebiet weiter entfernt ist. Daraus lässt sich für die Herausbildung von $\tilde{o}$ schlussfolgern, dass der Laut wohl von der Sprache mit der höchsten und regelmäßigsten Verbreitung (= Estnisch) ausgegangen ist und sich in vielen Belegen mit den beiden geographisch näher gelegenen Sprachen Wotisch und Südestnisch überschneidet, wenngleich die Ausprägung in diesen Sprachen bereits schwächer ist und $\tilde{o}$ seltener auftritt. Festgemacht werden kann diese Entwicklung nicht nur an den Entwicklungen von $* e$ und $* o$, sondern auch an den marginalen Vertretungen, in denen ein Lautwandel in diesen drei Sprachen vorliegt. Belege wie (127) est. sõna 'Wort' wot. sõna id. estS. sõna id. oder (64) est. nõmm, gen. -e 'Heide' wot. nõmmi, gen. nõmmõ õ id. estS. nõmm gen. $-e$ id. deuten auf einen engen Kontakt 
hin, doch auch Unterschiede sind zu bemerken: z.B. (60) est. nõel, gen. - $a$ 'Nadel' estS. nõgõl; nôkl, gen. nõgla id. wot. nigla id. Insgesamt ist die Schnittmenge der Entsprechungen in den drei Sprachen im Hinblick auf die Entwicklung der ersten Silbe sehr hoch. Dies deckt sich auch mit einigen von VIITSOS Erkenntnissen. Zwar beschreibt dieser keine Assimilationstendenzen in größerem Rahmen, aber seiner Auffassung nach ging der Dialektverbund des Estnischen und Wotischen aus einer Verbindung mit dem Südestnischen hervor und löste sich erst spät wieder auf, woraufhin die beiden Sprachen auch unabhängig voneinander weitere Innovationen entwickelt haben sollen (VIITSO 1978b: 94f.). Geht man also davon aus, dass die Lautwandel, insbesondere jene mit $*$, nach intensiven Sprachkontakten in den einzelnen Sprachen noch weiter fortgeschritten sind, ist ein Unterschied in der Ausprägung der Ausdehnung nur eine logische Schlussfolgerung.

Das Livische ist ein letzter Ausläufer und zeigt daher die wenigsten Belege mit $\tilde{o}$ in der ersten Silbe. Dies geht mit den konkurrierenden Lautwandeln einher, die im Kontext der lexikalischen Diffusion beschrieben wurden. Womöglich haben die Entwicklungen des $* o$ in der ersten Silbe dort bereits eingesetzt, als im Livischen $\tilde{o}$ als letzter Ausläufer der Entwicklung aufkam. Diese Vermutung wird durch frühere Erkenntnisse zum Livischen gedeckt: So schreibt SUHONEN (1988: 290), dass es „sich im Verlauf seiner geschichtlichen Entwicklung deutlich von seinen nächsten Verwandten entfernt hat“". Von einer frühen Abspaltung des Livischen, auch von den anderen ostseefinnischen Sprachen mit o, kann vor dem Hintergrund der untersuchten Belege ausgegangen werden. ${ }^{95}$

\footnotetext{
95 VIITSO (1978b: 98f., 104) beschreibt ebenso, dass das Livische sich früh von den anderen ostseefinnischen Sprachen abgespalten haben muss und einige eigenständige Entwicklungen vollzogen hat. Den Lautwandel $* o>$ uo bezieht er in seine Betrachtungen nicht mit ein, obwohl er für die Entwicklung der ersten Silbe im Livischen wesentlich ist. Eine frühe Abspaltung von den anderen ostseefinnischen Sprachen erwägt VIITSO auch für das Südestnische (ebd.). Diese Annahme mag richtig sein, zeigt sich aber vor allem in anderen Entwicklungen als der Entstehung des neuen Lautes. Betrachtet man die Verbreitung von õ genauer, so zeigt sich eine Überschneidung zwischen dem Süd- und dem Standardestnischen in diesem Korpus in einem recht großen Rahmen.
} 
Bereits bei der Betrachtung des wissenschaftsgeschichtlichen Hintergrundes dieser Fragestellung ist deutlich geworden, dass die Ansichten zu Alter und Entwicklung des Lautes $\tilde{o}$ schon von Beginn der Erforschung an sehr konträr waren. Diese Untersuchung hat nun einen Beitrag darin geleistet, zunächst einen allgemeinen und wissenschaftsgeschichtlichen Überblick über das Thema und dessen Erforschung zu geben und mit der Hilfe eines kommentierten Korpus eine Antwort auf die Frage nach der Entwicklung und dem Alter von õ zu suchen.

Rein wissenschaftsgeschichtlich betrachtet hat man es bei dieser Thematik mit einer langjährigen Kontroverse zu tun, für die bis dato noch keine allgemein gültige Erklärung gefunden wurde. Ebenso viele Forscher haben sich für wie gegen den Laut als sekundäre Entwicklung ausgesprochen, dafür und dagegen argumentiert und sich wissenschaftliche Streitigkeiten geliefert. Ein gemeinsamer Konsens konnte jedoch bislang nicht gefunden werden. Die Untersuchung hat versucht, nicht nur die wissenschaftsgeschichtlichen Aspekte darzulegen, sondern auch die wichtigsten Theorien anhand von sprachwissenschaftlichen Daten zu bewerten. Dabei hat sich gezeigt, dass die Annahme eines sekundären Lautwandels weitaus wahrscheinlicher ist als die eines primären Lautes $\tilde{o}$.

Im Rahmen der Erforschung von $\tilde{o}$ hat es bereits einige Erkenntnisse gegeben, die Untersuchung eines umfangreichen Korpus ist jedoch nur in den seltensten Fällen unternommen worden. Oft ist dieser für die Leser nicht nachvollziehbar und wurde von den jeweiligen Autoren nicht aufbereitet. Das liegt nicht zuletzt daran, dass es sich meist nur um Artikel zu besagter Fragestellung handelt, deren Rahmen in gewisser Hinsicht begrenzt ist. Als Monografie liegt lediglich die Arbeit von WIIK (Viron õ (Das estnische õ), 1986) vor, die jedoch, wie viele weitere Beiträge vor und nach ihr, nur das Estnische behandelt, wenngleich er sein Material offen darlegt. Eine umfassende Analyse mit kommentierten und für den Leser zugänglichen wotischen, livischen und südestnischen Daten konnte im Vorfeld dieser Arbeit nicht ermittelt werden und ist somit ein Novum auf diesem Gebiet.

Die Betrachtung des Korpus unter Berücksichtigung der dieser Untersuchung vorausgegangenen Arbeiten hat in vielerlei Hinsicht eine Erklärung für die Entwicklung des Lautwandels geben können und knüpft dabei in verschiedenen Punkten an die Erkenntnisse an, die bereits u.a. ITKONEN und VIITSO gewonnen haben. Es war möglich, eine Vielzahl an Annahmen und Hypothesen nicht nur für das Estnische, sondern auch für die anderen Sprachen zu bekräftigen, wie beispielsweise den Lautwandel $* e>\tilde{o}$ bei einem Hintervokal in der zweiten Silbe sowie 
den regelmäßigen Entwicklungen $* o u>\tilde{o u}$ und $* e u>\tilde{o u}$. Zum ersten Mal deutlich expliziert wurde im Hinblick auf $\tilde{o}$ die Sonderentwicklung des Livischen. Zwar ist aus früheren Arbeiten schon bekannt, dass es sich anders verhält als seine nahverwandten Sprachen, dass sein Sonderleben jedoch für $\tilde{o}$ von großer Wichtigkeit ist, wurde bisher nicht so umfassend festgehalten. An einigen Stellen konnten lautliche Gegebenheiten weiter aufgeklärt werden, für die es bisweilen zu wenige Informationen gab: So hat sich z.B. herausgestellt, dass der Vokal des Wortstamms urfi. *mett $V$ - des Belegs (10) est. mõtelda 'denken, nachdenken' wot. mõtôlla id. liv. mõtlõ id. estS. .mõtlõma, II. Infinitiv mõtõldaq id. wohl ein Hintervokal war, wenngleich jener auch in diesem Kontext nicht genauer bestimmt werden konnte. Nichtsdestoweniger ist die Betrachtung auch in diesem Rahmen in einigen Punkten an die Grenzen der möglichen Analyse gestoßen. In einem größeren Bereich konnten strukturierte Regeln festgehalten werden, warum aber die Lautwandel irgendwann versiegen und ihre Produktivität nachlässt, konnte nicht ergründet werden.

Folgende Punkte waren bereits aus dieser Untersuchung vorausgegangenen Arbeiten bekannt und konnten bestätigt werden:

- Der Laut $\tilde{o}$ ist eine jüngere Erscheinung, die es in der gemeinostseefinnischen Grundsprache noch nicht gegeben hat.

- Die Vokalharmonie in der ostseefinnischen Grundsprache ist Voraussetzung für die Entwicklung.

- Er kam wohl durch das verstärkte Auftreten von $a$ in der zweiten Silbe auf. Letzteres kam in den ostseefinnischen Sprachen v.a. durch die Entlehnungen aus dem Baltischen und Germanischen auf.

- Er ist wohl als früher datierbar als das Eintreten der slawischen Lehnwörter in die ostseefinnischen Sprachen, da diese kaum betroffen sind.

- Aufgrund der regelmäßigen Entwicklung ist anzunehmen, dass *e als Ausgangspunkt für den Lautwandel im Zentrum steht.

Zu dieser Liste sind als Ergebnis der Betrachtung zu ergänzen:

- Der neue Laut $\tilde{o}$ hat sich wohl vom Estnischen aus verbreitet, da diese Sprache am stärksten und gleichmäßigsten von den Veränderungen betroffen ist.

- Im Hinblick auf $* e$ und $*_{o}$ in der ersten Silbe ist er sehr expansiv, während er es bei anderen Lauten nicht ist. 
- $\quad$ Ein Lautwandel $* o>\tilde{o}$ tritt bei illabialen Vokalen in der Folgesilbe auf.

- Der Lautwandel ist nicht mehr produktiv, da Wörter, die die Bedingungen erst später erfüllen, nicht mehr erfasst werden. Ferner ist die Entwicklung von *o in der ersten Silbe nicht abgeschlossen und führt hier zu einem dualen Verhältnis im Wortschatz des Estnischen, Wotischen und Südestnischen (lexikalische Diffusion).

- Eine frühe Abspaltung des Livischen von den anderen ostseefinnischen Sprachen der Südgruppe lässt sich aufgrund sprachinterner Entwicklungen bestätigen. Im Hinblick auf die Entwicklung von $*_{o}$ ist der Lautwandel in dieser Sprache die Ausnahme, da andere Entwicklungen wie $*_{o}>$ uo die Regel sind.

Festzuhalten ist, dass die Wissenschaft in dieser Fragestellung an einen Punkt gekommen ist, an dem sie aufgrund des kaum mehr erweiterbaren Sprachmaterials wohl kaum noch neue Erkenntnisse über die Entwicklung des Lautes gewinnen kann. Fraglich ist, ob ein genauerer Blick in die Dialektologie der Einzelsprachen vielleicht weitere Impulse geben kann. Eine Lösung des Problems auf Grundlage einzelner Dialekte ist jedoch vor dem Hintergrund der bisher erarbeiteten Datenlage eher unwahrscheinlich und würde höchstens über die Entwicklung innerhalb der jeweiligen Varietäten Aussagen treffen können. An relevanten Stellen wurden die Dialekte in dieser Arbeit bereits berücksichtigt und konnten zumindest in einzelnen Punkten die Verhältnisse aufklären.

Obwohl in dieser Untersuchung Lösungsansätze für die Frage nach der Entwicklung von $\tilde{o}$ aufgezeigt werden konnten, sind noch immer einige Aspekte unbeantwortet, die sich auch mit weiterer Recherche in einem solchen Rahmen nicht auflösen lassen. Da davon ausgegangen werden kann, dass der Laut wohl eine sekundäre und recht lokale Entwicklung in der Südgruppe der ostseefinnischen Sprachen ist, scheint ein Einbeziehen der anderen finnougrischen Sprachen außerhalb des Ostseefinnischen nur bedingt zielführend. Über diese könnten kaum neue Erkenntnisse der lautlichen Umgebung der Sprachen gewonnen werden, die nicht ohnehin bereits in die Analyse eingeflossen sind. Über die lautlichen Verhältnisse im Marischen, Mordwinischen und Samischen referierte schon ITKONEN (1945) in seinem im wissenschaftsgeschichtlichen Teil ausführlich behandelten Artikel ausreichend. Fortsetzer eines Lautes $\tilde{o}$ aus einer uralischen Sprachstufe hat es bis ins Ostseefinnische wohl nicht gegeben, sodass kein Grund besteht, ihn als ursprünglich vorauszusetzen. Es bleibt daher also nur, die weitere Entwicklung der vom Lautwandel betroffenen Sprachen abzuwarten, insbesondere der des Estnischen, das über eine hohe Sprecheranzahl und somit größere potenzielle Datenmengen verfügt. Dabei 
sollte man vor allem die Entwicklung von $* o$ verfolgen, um zu sehen, ob sich daraus neue Erkenntnisse gewinnen lassen. Möglicherweise können sie Aufschluss darüber geben, ob sich der Lautwandel heute wider Erwarten noch weiter ausdehnt oder ob er seinen Zenit bereits erreicht hat, wie aufgrund der dargestellten Diskussion anzunehmen ist. Wie lange ein derartiger Prozess dauern würde, ist schwer vorherzusagen. Aufgrund der Darstellung hier kann man jedoch davon ausgehen, dass es wenigstens Jahrzehnte sind, wenn er nicht sogar noch größere Zeitfenster umfasst. 
8. Literaturverzeichnis

ALS = VIIKBERG, Jüri. 2016. Alamsaksa laensõnad eesti keeles. http://www.eki.ee/dict/asl/index.cgi (Abgerufen am 18.09.2020)

ALVRE, Paul.

1976. Arvo Laanest, Sissejuhatus läänemeresoome keeltesse, Tallinn 1975. 240 S. In: Sovetskoe finno-ugrovedennie. XII, 3. Tallinn. 214-225.

ARISTE, Paul.

1968. A Grammar of the Votic Language. Bloomington/The Hague. Indiana University Publications. Uralic and Altaic Series. Volume 68.

Asu, Eva Liina \& Teras, Pire.

2009. Estonian. In: Journal of the International Phonetic Association, 39(3), 367-372.

doi:10.1017/S002510030999017X

(Abgerufen am 30.09.2021)

AuTIO, Veli-Matti.

1998. Setälä, Emil Nestor (1864-1935). Suomen kielen ja kirjallisuuden professori, ministeri, lähettiläs, valtioneuvos.

https://kansallisbiografia.fi/kansallisbiografia/henkilo/500

(Abgerufen am 12.03.2018)

BLOKLAND, Rogier.

2009. The Russian loanwords in Literary Estonian. Wiesbaden. Veröffentlichungen der Societas Uralo-Altaica 78.

BLUMBERGA, Renāte 2011.

Liivlased 19.-21. sajandil. In: BLumBERGA, Renāte; MäKELÄINEN, Tapio; PAJUSALU, Karl (Hrsg.). 2011. Liivlased: ajalugu, keel ja kultuur. Tallinn. 127-153.

BÖGER, Joachim [et al.]. 2008. = BÖGER, Joachim; DIEKMANN, Helmut; LENK, Hartmut; SCHRÖDER, Caren; KÄRNÄ, Aino. ${ }^{12} 2008$. Suomi-saksa-suomi-sanakirja. Helsinki. 
CASTRÉN, Jalmar [et al.]. 1917. = CASTRÉN, Jalmar; ForSMAN, Jaakko; GROTENFELT, Kustavi; Hendell, Lauri; HJelt, Edvard; SAXÉn, Uno; SEtÄlä, E. N.; VÄLIKANGAS, Ilmari; WiChMAN, Yrjö (Hrsg.). 1917. Tietosanakirja IX. Stambulov - Työaika. Helsinki.

CASTRÉn, Jalmar [et al.]. 1919. = CASTRÉN, Jalmar; ForSMAn, Jaakko; GrotENFELT, Kustavi; SetÄlÄ, E. N.; SAXÉn, Uno; VälikAngas, Ilmari; Wichman, Yrjö (Hrsg.). 1906. Tietosanakirja X. Työehtosopimus - Öölanti. Helsinki.

COLLINDER, Björn.

1960. Comparative grammar of the Uralic languages. Stockholm.

COMRIE, Bernard.

1988. General Features of the Uralic Languages. In: Sinor, Denis (Hrsg.). The Uralic Languages. Description, History and Foreign Influences. Handbuch der Orientalistik. Volume I. Leiden/New York/København/Köln. 451-477.

DÉCSY, Gyula.

1965. Einführung in die finnisch-ugrische Sprachwissenschaft. Wiesbaden.

DINI, Pietro U.

2014. Foundations of Baltic Languages. Vilnius.

EES = MetsmäGI, Iris; Sedrik, Meeli; SoosaAr, Sven-Erik. 2012. Eesti etümoloogiasõnaraamat. Tallinn.

EEW I-XII = MÄGISTE, Julius. 1982-1983. Estnisches Etymologisches Wörterbuch . I-XII. Helsinki.

EK jallisuuden Seuran Toimituksia 156. Helsinki.

EKG = ERELT, Mati (Hrsg.) [et. al.]. 1995. Eesti Keele Grammatika I. Morfoloogia. Sõnamoodustus. Tallinn.

EKKS = KAALEP, Heiki-Jaan; MuISCHNEK, Kadri. 2002. Eesti kirjakeele sagedussõnastik. Tartu.

http://hdl.handle.net/10062/54930 (Abgerufen am 22.09.2020)

EMS

= 1994-. Eesti murrete sõnaraamat I-. Tallinn.

http://www.eki.ee/dict/ems/pdf.html (Abgerufen am 17.06.2020)

ERNITS, Enn.

2009. Vadja haritlane Dmitri Tsvetkov. Tartu. 

2014. Eesti-võru sõnaraamat. Võru.

FORSSMAN, Berthold.

2005.

2010. GENETZ, Arvid.

1877. 1897. Versuch einer karelischen Lautlehre. Helsingfors.

Ensi tavuun vokaalit suomen, lapin ja mordvan kaksi-ja useampitavuissa sanoissa. In: Suomi. Kirjoituksia isänmaallisista aineista. 13, 3. Helsinki. $1-56$.

HAKULINEN, Lauri.

${ }^{4} 1979$.

Suomen kielen rakenne ja kehitys. Helsinki.

HALL, T. Alan.

2011.

Phonologie. Eine Einführung. Berlin/New York.

HoLST, Jan Henrik.

2001.

IMS

\section{ITKONEN, Erkki.}

1945.

1946.

JANHUNEN, Juha.

1975-1976. Adalékok az északi szamojéd hangtörténethez: Vokalizmus. In: Néprajz

KaLlio, Petri.

2014. és nyelvtudomány. XIX-XX. Szeged. 165-188.

Die Herkunft des estnischen Vokals õ. In: Journal de la Société FinnoOugrienne 89. Helsinki. 57-98.

= NIRVI, R. E. (Hrsg.). 1971. Inkeroismurteiden sanakirja. Helsinki. Lexica Societatis Fenno-Ugricae XVIII.

Onko kantasuomessa ollut keskivokaaleja? In: Virittäjä 49. Helsinki. 158-182.

Zur Frage nach der Entwicklung des Vokalismus der ersten Silbe in den finnisch-ugrischen Sprachen, insbesondere im Mordwinischen. In: Finnisch-Ugrische Forschungen 29. Helsinki. 222-337.

The Diversification of Proto-Finnic. In: Ahola, Joonas; Frog; Tolley, Clive (Hrsg.). 2014. Fibula, Fabula, Fact. The Viking Age in Finland. Studia Fennica Historica 18. Helsinki. 155-168. 
KAnN [et al.]. 1972. = KAnN, K.; KibBERMAnN, E.; KibBERMAnN, F.; KIROTAR, S. ${ }^{3} 1972$. Estnisch-deutsches Wörterbuch. Tallinn.

KANN. [et al.] 2003. = KANN, Kallista; KIBBERMANN, Elisabeth; KIBBERMANN, Feliks; KIROTAR, Salme. ${ }^{5}$ 2003. Eesti-saksa sõnaraamat. Estnisch-deutsches Wörterbuch. Tallinn.

KÄSI, Inge.

2016. Seto sõnastik. Tallinn.

http://www.eki.ee/dict/setosonastik/Seto_sonastik.pdf

(Abgerufen am 19.06.2018)

KASIK, Reet.

2015. Sõnamoodustus. Tartu. Eesti Keele Varamu I.

KASK, Arnold.

1972. Eesti keele ajalooline grammatika. Häälikulugu. Tartu.

KATARA, Pekka; SchellbaCH-KopRA, Ingrid.

${ }^{5}$ 1974. Suomalais-Saksalainen Suursanakirja. Finnisch-Deutsches Großwörterbuch. Porvoo/Helsinki.

KEEM $2002=$ KEEM, Hella; KÄSI, Inge. 2002. Võru murde tekstid . Eesti murded VI. Tallinn.

KEEM, Hella.

1997. Võru keel. Tallinn.

KETTUNEN, Lauri.

1948. Eikö kantasuomessa ole ollut keskikielen vokaaleja? In: Virittäjä 52. Helsinki. 120-124.

1986. Vatjan kielen Mahun murteen sanasto. Castrenianum toimitteita 27. Helsinki.

KoKLA [et al.]. 1972. = KoKLA, Paul; LAANPERE, Helga; MÄGER, Mart; PIKAMÄE, Arno. 1972. Eesti-soome sõnaraamat. Tallinn.

LAANEST, Arvo.

1982. $\quad$ Einführung in die ostseefinnischen Sprachen. Hamburg.

LÄGlos I-III = KYlstra, A. D.; HAHMO, Sirkka-Liisa; HoFstra, Tette; NikKILÄ, Osmo. 1991-2012. Lexikon der älteren germanischen Lehnwörter in den ostseefinnischen Sprachen. Bd. I-II: Amsterdam/Atlanta, GA. Bd. III: Amsterdam/New York, NY. 
LAUERMA, Petri.

1993. Vatjan vokaalisointu. Mémoires de la Société Finno-Ougrienne 214. Helsinki.

LEHTISALO, T.

1936. Über die primären ururalischen Ableitungssuffixe. Mémoires de la Société Finno-Ougrienne LXXII. Helsinki.

LELS

= VIITSO, Tiit-Rein; ERNŠTREITS, Valts. 2012. Lìvõkīel-ēstikīel-leţkīel sõnārōntõz. Liivi-eesti-läti sõnaraamat. Lībiešu-igaunu-latviešu vārd$n \bar{i} c a$. Tartu/Rìga.

LW = KeTTUNEN, Lauri. 1938. Livisches Wörterbuch. Mit grammatischer Einleitung. Lexica Societas Finno-Ugricae 5. Helsinki.

Must, Mari.

2000. Vene laensõnad eesti murretes. Tallinn.

NiKOLAEVA, I. A.

1997.

= Николаева, И. А. 1997. Водский язык. In: Ярцева, В.Н. [et.al.]. Языки российской федерации и соседних государств. І. А-И. 267272.

PAJUSALU, Karl.

2012. Phonological Innovations of the Southern Finnic Languages. In: Mémoires de la Société Finno-Ougrienne 266. Helsinki. 201-224.

2014. The relationship between Salaca Livonian and Courland Livonian dialects. In: Eesti ja soome-ugri keeleteaduse ajakiri 5, 1. Tartu. 149-171.

PARVE, Merike.

2000. Võru lühikeste monoftongide akustikast. In: PAJUSALU, Karl; PARVE, Merike; TerAs, Pire; IVA, Sulev. 2000. Võru Vokaalid I. Tartu Ülikooli Eesti Keele Õppetooli Toimetised 13. Tartu. 9-38.

PosTI, Lauri.

1942. Grundzüge der livischen Lautgeschichte. Helsinki. Mémoires de la Société Finno-Ougrienne LXXXV.

RAUN, Alo.

1971. Essays in Finno-Ugric and Finnic linguistics. Indiana University Publications - Uralic and Altaic Series. Volume 107. Bloomington/The Hague. 
SAMMALlAHTI, Pekka.

1979. Über die Laut- und Morphemstruktur der uralischen Grundsprache. In: Finnisch-Ugrische Forschungen XLIII. Helsinki. 22-66.

SCHMIDT, Johannes.

1872. Die Verwandtschaftsverhältnisse der indogermanischen Sprachen. Weimar.

SETÄLÄ, Eemil Nestor.

1896. Über Quantitätswechsel im Finnisch-Ugrischen. Vorläufige Mitteilung. In: Journal de la Société Finno-Ougrienne XIV 3 . Helsinki. 1-54.

1899. Yhteissuomalainen äänehistoria. I ja II vihko. Helsinki.

SJÖGREN, Johan Andreas.

1861. Joh. Andreas Sjögren's Gesammelte Schriften. Band II. Teil II. Livischdeutsches und deutsch-livisches Wörterbuch. Im Auftrage der Kaiserlichen Akademie der Wissenschaften bearbeitet von Ferdinand Joh. Wiedemann. St. Petersburg.

SKES I-VII = ToIVONEN, Y. H. [et.al.]. 1955-1981. Suomen kielen etymologinen sanakirja I-VII. Helsinki. Lexica Societatis Fenno-Ugricae XII.

STEINITZ, Wolfgang.

1944. Geschichte des finnisch-ugrischen Vokalismus. Acta Instituti Hungarici Universitatis Holmiensis. Series B. Linguistica 2. Stockholm.

SSA I-III = ITKONEN, Erkki. KuLONEN, Ulla-Maija. (Hrsg.) [et. al.]. 1992-2000. Suomen sanojen alkuperä. Etymologinen sanakirja. I-III. Helsinki.

SST = Suomen sanomalehden taajuussanasto.

https://korp.csc.fi/suomen-sanomalehtikielen-taajuussanasto-B9996.txt (Abgerufen am 22.09.2020)

SUHONEN, Seppo.

1988. Geschichte der ostseefinnischen Sprachen. In: SinOR, Denis (Hrsg.). The Uralic Languages. Description, History and Foreign Influences. Handbuch der Orientalistik. Volume I. Leiden/New York/København/Köln. 288-313. 
TERAS, Pire.

2003. Lõunaeesti vokaalisüsteem: Võru pikkade vokaalide kvaliteedi muиtumine. Tartu. Dissertationes Philolohiae Estonicae Universitatis Tartuensis 11.

THOMSEN, Vilhelm.

1890. Beröringer mellem de finske og baltiske (litauisk-lettiske) sprog: en sproghistorisk undersøgelse. København.

1931. Samlede Afhandlinger. Fjerde Bind. Berührungen zwischen den finnischen und den baltischen (litauisch-lettischen) Sprachen. Eine sprachgeschichtliche Untersuchung. København.

TRASK, R. L.

2002. Historical Linguistics. London.

UEW I-III = RÉDEI, Károly. [et. al.]. 1986-1991. Uralisches Etymologisches Wörterbuch. Band I-III. Budapest.

UNIVERE, Aili.

1986. Idamurre. In: Emakeele Seltsi Aastaraamat 32. 59-93.

VES = JÜVÄ, Sullõv. 2002. Võro-eesti-synaraamat. Võru-eesti sõnaraamat. Võru Instituudi toimetised 12. Tarto/Võro.

Sowie auf http://www.folklore.ee/Synaraamat und https://synaq.org (Abgerufen am 22.08.2020)

VIITSO, Tiit-Rein.

1961. Vadja keele Luutsa-Liivtšüla murraku fonoloogia. In: Emakeele Seltsi Aastaraamat VII. 142-174.

1978a. Läänemeresoome esimese silbi õ ajalugu. In: Keel ja Struktuur 10. 1978. Tartu. 81-117.

1978b. The history of Finnic $\tilde{o}$ in the first syllable. In: Sovetskoe finnougrovedennie. XIV, 2. 1978. Tallinn. 86-106.

1998a. Fennic. In: ABONDOLO, Daniel (Hrsg.). 1998. The Uralic Languages. London/New York. 96-114.

1998b. Estonian. In: ABondolo, Daniel (Hrsg.). 1998. The Uralic Languages. London/New York. 115-148. 
2003. Phonology, Morphology and Word Formation. In: ERELT, Mati (Hrsg.). 2003. Estonian Language. Linguistica Uralica. Supplementary Series. Volume 1. Tallinn. 9-92.

2011. Liivi keele põhijooned. In: BLUMBERGA, Renāte; MÄKELÄINEN, Tapio; Pajusalu, Karl (Hrsg.). 2011. Liivlased: ajalugu, keel ja kultuur. Tallinn. 203-217.

VISK

= HaKulinen, Auli; Vilkuna, Maria; Korhonen, Riitta; KoIVIsto, Vesa; HeInONEn, Tarja Riitta; AlHo, Irja. 2004. Iso suomen kielioppi. Helsinki. Online-Ausgabe.

http://scripta.kotus.fi/visk/etusivu.php (Abgerufen am 27.08.2020)

VKÄ = KetTUnen, Lauri. $1930 .^{2}$ Vatjan kielen äänehistoria. Suomalaisen Kirjallisuuden Seuran Toimituksia 185. Helsinki.

VKS

= GRÜNBERG, Silja (Hrsg.). 2013. Vadja keele sõnaraamat. Vaddaa tšeelee sõna-tširja. Slovar' vodskogo jazyka. Tallinn.

VMS II = PALL, Valdek (Hrsg.). 1989. Väike murde sõnastik II. Tallinn.

WIEDEMANN, F.J.

1864.

Versuch ueber den werroehstnischen Dialekt. St. Petersburg.

1923. Eesti-Saksa Sõnaraamat. Estnisch-deutsches Wörterbuch. Dritter unveränderter Druck nach der zweiten, von Dr. Jakob Hurt redigierten Auflage. Tartu.

WIIK, Kalevi.

1986.

Viron õ. Turun Yliopiston Suomalaisen ja Yleisen Kielitieteen Laitoksen Julkaisua 30. Turku.

WINKLER, Eberhard.

1997.

Krewinisch. Zur Erschließung einer ausgestorbenen ostseefinnischen Sprachform. Wiesbaden. Veröffentlichungen der Societas Uralo-Altaica 49.

2002a. Estnisch. In: OKUKA, Miloš (Hg.). 2002. Wieser Enzyklopädie des europäischen Ostens. Band 10: Lexikon der Sprachen des europäischen Ostens. Klagenfurt. 633-645.

https://eeo.aau.at/eeo.aau.at/indexbc6e.html?title=Sprachenlexikon (Abgerufen am 16.10.2020) 
2002b. Wotisch. In: OKUKA, Miloš (Hg.). 2002. Wieser Enzyklopädie des europäischen Ostens. Band 10: Lexikon der Sprachen des europäischen Ostens. Klagenfurt. 761-763.

https://eeo.aau.at/eeo.aau.at/indexbc6e.html?title=Sprachenlexikon (Abgerufen am 01.10.2021)

2010. Zum Wortschatz einer sterbenden Sprache-Salis-Livisch. In: SAARINEN, Sirkaa; SirTonen, Kirsti; VAITTINen, Tanja (Hrsg.). 2010. Sanoista kirjakieliin. Juhlakirja Kaisa Häkkiselle 17. marraskuuta 2010. Mémoires de la Société Finno-Ougrienne 259. Helsinki. 371-382.

2011. Laensõnakihtidest liivi keeles. In: BLUMBERGA, Renāte; MÄKELÄINEN, Tapio; PAJUSALU, Karl (Hrsg.). 2011. Liivlased: ajalugu, keel ja kultuur. Tallinn. 231-237.

WINKLER, Eberhard; PAJUSALU, Karl.

2009. Salis-livisches Wörterbuch. Linguistica Uralica. Supplementary Series. Volume 3. Tallinn. 


\begin{tabular}{|c|c|c|c|}
\hline ADESS & Adessiv & ing. & ingrisch \\
\hline AKK & Akkusativ & INTR & intransitiv \\
\hline $\mathrm{AKT}$ & aktiv & kar. & Karelisch \\
\hline altnord. & altnordisch & KомР & Komperativ \\
\hline altruss. & altrussisch & Kur. & Kurland-Livisch \\
\hline ar. & arisch & KurW. & Kurland-Livisch (West) \\
\hline balt. & baltisch & KurO. & Kurland-Livisch (Ost) \\
\hline baltendt. & baltendeutsch & Kukk. & Kukkuzi (wot. Dialekt) \\
\hline bzgl. & bezüglich & lett. & lettisch \\
\hline dial. & dialektal & lit. & litauisch \\
\hline dt. & deutsch & liv. & livisch \\
\hline ELAT & Elativ & livO. & Ost-Livisch \\
\hline est. & estnisch & livW. & West-Livisch \\
\hline estN. & nordestnische Dialekte & $\operatorname{lp}$ & samisch \\
\hline estS. & südestnisch & lpN. & Nordsamisch \\
\hline fi. & finnisch & lüd. & lüdisch \\
\hline fiugr. & finnougrisch & Luu. & Luuditsa (wot. Dialekt) \\
\hline fosfi. & frühostseefinnisch & md. & mordwinisch \\
\hline frühgerm. & frühgermanisch & mdE. & erza-mordwinisch \\
\hline frühindoiran. & frühindoiranisch & mnd. & mittelniederdeutsch \\
\hline frühuriran. & frühuriranisch & n.Chr. & nach Christus \\
\hline frühurfi. & frühurfinnisch & nd. & niederdeutsch \\
\hline frühurgerm. & Frühurgermanisch & neuschwed. & Neuschwedisch \\
\hline frühurosfi. & frühurostseefinnisch & NoM & Nominativ \\
\hline gen. & Genitiv & obugr. & obugrisch \\
\hline germ. & germanisch & od. & oder \\
\hline IMP & imperfekt & onomat. & onomatopoetisch \\
\hline indoeur. & indoeuropäisch & osfi. & ostseefinnisch \\
\hline indoiran. & Indoiranisch & PART & Partitiv \\
\hline IND & Indikativ & PL & Plural \\
\hline INESS & inessiv & perm. & permisch \\
\hline
\end{tabular}




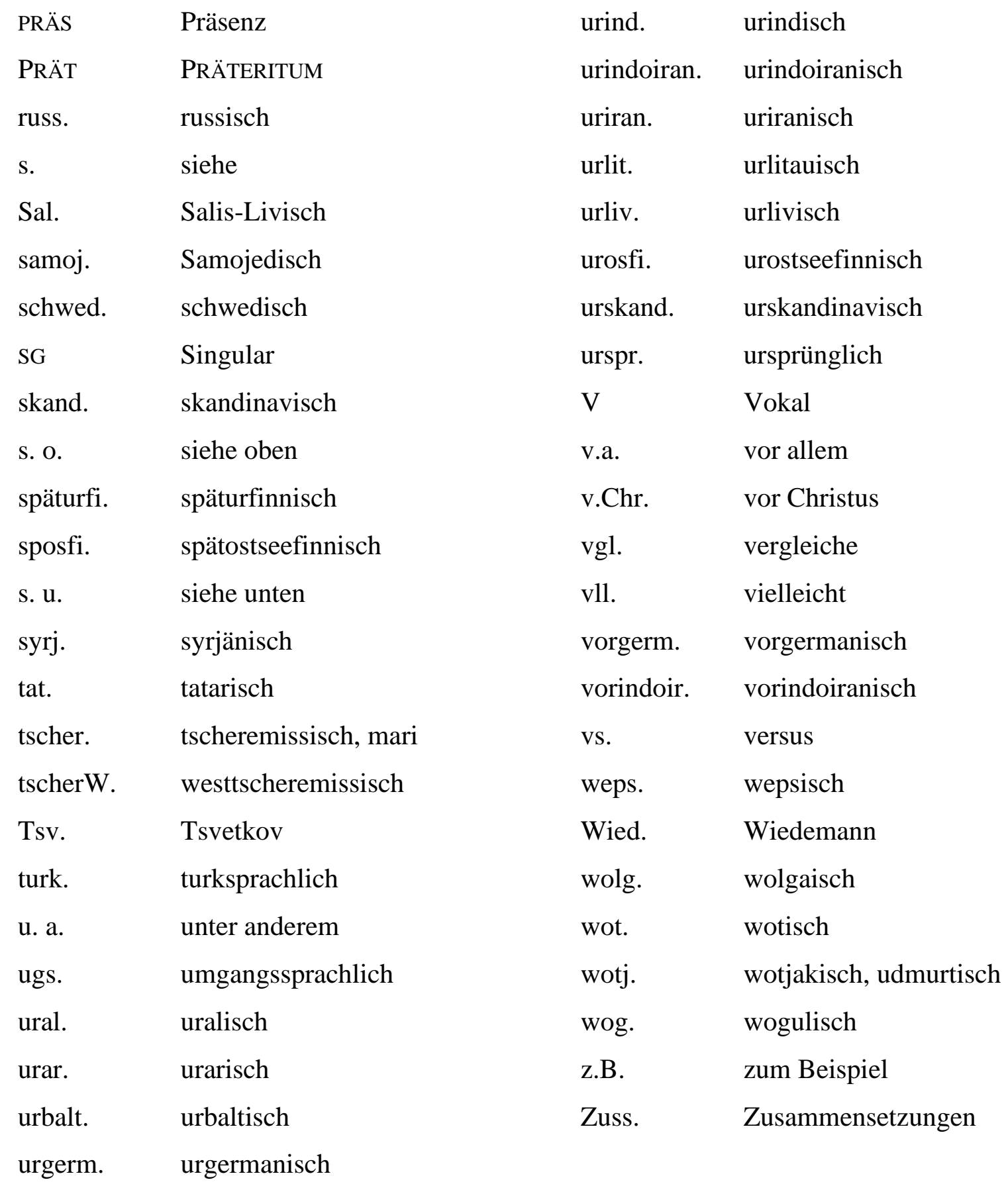




\section{Anhang I: Nicht behandelte Belege}

Der vorliegende Anhang gibt einen Überblick über die Belege, in denen zwar $\tilde{o}$ in der ersten Silbe auftritt, die aber dennoch gemäß der Kapitel 1.3.1 angeführten Bedingungen aus der Untersuchung ausgeschlossen wurden. Nicht behandelt werden Wörter ohne etymologischen $\mathrm{Zu}$ sammenhang zu anderen Wörtern, derivierte Wörter sowie künstliche Bildungen und Wörter mit unklarer Etymologie. Auch ausgeschlossen wurden russische Lehnwörter mit $b l$ in der ersten Silbe und niederdeutsche Entlehnungen. Nicht aufgelistet werden an dieser Stelle ferner Ableitungen im Korpus behandelter oder in dieser Auflistung angeführter Wörter (z.B. est. jõhvikas 'gewöhnliche Moosbeere' zu est. jõhv 'Pferdehaar, Rosshaar'), zusammengesetzte Formen, deren einschlägiger Bestandteil bereits behandelt wurde z.B. wot. jõgõoranta 'Flussufer' (vgl. wot. jõtši, gen. jõgõ̃ 'Fluss') oder Varianten.

Die Belege werden so notiert, wie sie in den Quellen auftreten, im Südestnischen z.B. $y \sim \tilde{o}$ für jene, die nur in VES vorkommen.

\section{ESTNISCH}

bõliina 'Bylina, russ. Heldenepos' < russ. былина (BLOKLAND 2009: 82)

hõigata 'rufen' keine Zusammenstellung mit anderen osfi. Sprachen (EES 82)

hõissa (Ausruf des fröhlichen Jubels) wohl < dt. heisa, heissa, keine Zusammenstellung mit anderen osfi. Sprachen (EES 82)

hõivata 'besetzen, einnehmen; in Besitz nehmen, okkupieren' künstlich gebildeter Stamm (EES 82)

hõllandus 'Sehnsucht' Etymologie unsicher (EES 82)

hõlst, gen. hõlsti 'leichtes weites Überkleid' Etymologie unsicher (SSA I: 192; EES 82f.); vgl. auch BLOKLAND (2009: 92ff.)

hõlvata 'einnehmen, umfassen' künstlich gebildeter Stamm (EES 83)

hõre 'undicht, spärlich, dünn' wohl Vermischung der Stämme est. harv 'selten; undicht, spärrlich' und sõre 'undicht, grob' (EES ), von welchen letzterer im Korpus behandelt wird

hõrnas 'Bachforelle (Salmo trutta fario)' keine Verbindung zu anderen osfi. Belegen, Etymologie unklar (EES 83)

jõllis 'hervorgequellen, aufgerissen (Augen)' keine Verbindung zu anderen osfi. Belegen (EES 103) 
jõma 'unklare Stimmen, unklares Dröhnen' keine Verbindung zu anderen osfi. Belegen (EES 103)

jõmm, gen. jõmmi 'Dickerchen; kleiner Junge, kleines Kind' keine Verbindung zu anderen osfi. Belegen; Variante zu est. jumikas 'Skabiosen-Flockenblume (Centaurea scabosa)' unsicher (SSA I: 247; EES 101, 103)

jõmpsikas '(kleiner) Junge' keine Verbindung zu anderen osfi. Belegen (EES 103)

jõngermann 'kleiner Junge, kleines Kind' keine Verbindung zu anderen osfi. Belegen; < dt. junger Mann (EES 103)

jõngutada 'vor- und zurückbewegen, schaukeln' Etymologie unsicher (EES 103; in SSA nicht behandelt)

jõnk, gen. jõngi 'überernst, zornig, grob' keine Verbindung zu anderen osfi. Belegen (EES 103) jõnks, gen. jõnksu 'plötzliche Bewegung; Krümmung' Verweis auf est. jonks 'plötzliche Bewegung; Krümmung', welches keine Verbindung zu anderen osfi. Belegen vorweist (EES 98, 103) jõõr, gen. jõõra 'Geilheit, Wollust, Brünstigkeit' < baltendt. Gier, keine Verbindung zu anderen osfi. Belegen (EES 104)

jõss, gen. jõssi 'klein und korpulent; kleiner und korpulenter Junge oder Mann' keine Verbindung zu anderen osfi. Belegen (EES 103)

jõudsin 'Schwan' poetische Sprache (SSA I: 244)

klõmmida 'wiederholend hart schlagen, schleudern oder ziehen; hämmern, prügeln' Verweis auf est. plõmmia (s.u.), welches keine Verbindung zu anderen osfi. Belegen aufweist (EES 167) kõblas 'Hacke, Picke' < balt., keine Verbindung zu anderen osfi. Belegen (EES 198)

kõder, gen. kõdra 'Schote' Entsprechung zu anderen osfi. Sprachen unsicher (EES 198; in SSA nicht behandelt)

kõdu 'Moder' Variante von Stamm est. kaduda 'verloren gehen, verschwinden', wo es nicht weiter behandelt wird (EES 112f., 198)

kõhe 'unbehaglich, kühl, kalt, frostig' Etymologie unsicher (EES 198)

kõhelda 'unschlüssig sein, zaudern' wohl Variante von est. dial. kahkvel 'unentschlossen, aufgeregt (Angst)' dessen Etymologie unsicher ist (EES 116, 198)

kõhr, gen. kõhre 'Knorpel' Variante von est. kohr 'Ausbeulung, Schwellung', wo es nicht weiter behandelt wird (EES 170, 199)

kõhvitseda 'kratzen, schaben' < nd. schaven (EES 199)

kõkats 'unsicheres Objekt mit leichtem Bau' keine Verbindung zu anderen osfi. Sprachen (EES 199) 
kõksida 'leicht schlagen, gegeneinander zerschlagen, hämmern' Verweis auf est. koksida 'gegeneinander zerschlagen (Eier)', dort keine gesicherte Verbindung zu anderen osfi. Sprachen (EES 199)

kõld, gen. kõlla 'dünnes Leder, Membran' unsichere Etymologie (EES 200, vgl. SSA I: 341f.) kõle(kepp) 'großer, langer Spazierstock, Bettelstab' Zusammenstellung mit anderen osfi. Sprachen unsicher (SSA I: 388)

kõliseda 'klingen, schallen, tönen, läuten' Etymologie unklar (EES 200)

kõlkuda 'herab-, herunterhängen; sich schaukeln, wiegen; baumeln' möglicherweise gleich wie est. kõliseda (s.o.) (EES 200)

kõlu 'Hülse, Schale' unklare Etymologie (SSA I: 292; EES 200)

kõnd, gen. kõnna (alt.) 'Geld, Vermögen, Reichtum' unbekannte Herkunft (EES 200)

kõne 'Erzählen, Sprache, Vortrag' Etymologie unsicher (SSA I: 398; EES 201)

kõnts, gen. kõntsa 'Schlamm, Schlick' Etymologie ungesichert (EES 201)

kõõoksuda 'aufstoßen, rülpsen' keine Zusammenstellung mit anderen osfi. Sprachen (EES 203) kõõluda 'faulenzen, schaukeln, schwingen' keine Zusammenstellung mit anderen osfi. Sprachen (EES 203)

kõõpida 'mit einem scharfen Gegenstand glatt oder sauber schaben' keine Zusammenstellung mit anderen osfi. Sprachen (EES 204)

kõrts, gen - $i$ 'Kneipe, Krug, Schenke' < russ. корчма 'Kneipe', laut EES (202) seien Entsprechungen der anderen osfi. Sprachen < est.; vgl. auch BLOKLAND (2009: 146)

kõrtsmik 'Kneipenwirt' < russ. корчемник, keine Zusammenstellung mit anderen osfi. Sprachen (EES 202); vgl auch BLOKLAND (2009: 147f.)

kõrvits 'Kürbis (Cucurbita)' < nd. korves(e), korvisch, korvetze, korbitze, körwitze 'Kürbis' (EES 203)

kõss, gen. kõssu 'sehr leise Stimme oder Geräusch' keine Zusammenstellung mit anderen osfi. Sprachen (EES 203)

kõuts, gen. kõutsi 'Kater' < lett., keine Zusammenstellung mit anderen osfi. Sprachen (EES 203)

krõbe 'alter Mensch, Greis' Bildung aus est. kõbi 'Greis' und est. robiseda 'rauschen, rascheln' (EES 185)

krõhva '(magere) böse Frau' keine Verbindung zu anderen osfi. Belegen (EES 185)

krõll, gen. krõlli 'Silberschmuck mit großer Höhlung' keine Verbindung zu anderen osfi. Belegen (EES 185)

krõõt, gen. krõõda '(alte) hässliche Frau'< nd. Greet (Frauenname) (EES 185) 
lõhverdada 'schwatzen, plappern, schnattern' keine Zusammenstellung mit anderen osfi. Sprachen (EES 258)

lõit, gen. lõida 'Mittel zur Verbreitung von Informationen, Kommunikationsmittel' Etymologie unklar, keine Zusammenstellung mit anderen osfi. Sprachen (EES 258)

lõiv, gen. lõivu 'Gebühr' Zusammenstellung mit anderen osfi. Sprachen trotz Zusammenstellung laut EES (258) unsicher (vgl. SSA II: 36)

Iõmm, gen. lõmmu 'Holzscheit, gespaltenes Scheit' keine Zusammenstellung mit anderen osfi. Sprachen (EES 259)

lõõm, gen. lõ̃ma 'Glut, Lohe' Etymologie unsicher (EES 261)

lõõpida 'dumme Witze reißen' keine Zusammenstellung mit anderen osfi. Sprachen (EES 261)

lõõr, gen. $-i$ 'Feuerzug, Heizkanal, Rauchabzugskanal' < nd. rōr 'Schilfrohr, Rohr' (EES 261)

lõõrikas 'Regenwurm' (nordöstliche Dialekte) ? < fi. (SSA II: 70)

lõpus 'Kieme' Verbindungen zu anderen osfi. Sprachen unsicher, möglicherweise mit Stamm von est. lõppeda 'beenden' vermischt (EES 259)

lõrpida 'flüssige Gerichte (gierig) essen, (gierig) trinken, schlürfend essen oder trinken' (ugs.) keine Zusammenstellung mit anderen osfi. Sprachen (EES 259f.)

lõsn, gen. lõsna 'Tragbalken des Dreschzimmers' Stamm unbekannter Herkunft (EES 260)

lõss, gen. lõssi 'Magermilch, entrahmte Milch' keine Zusammenstellung mit anderen osfi. Sprachen (EES 260)

lõuend 'Leinwand, Leinen' < nd. louwent, louwant 'Leinwand' (EES 260)

lõusk, gen. lõusa 'Schnauze' künstlich gebildeter Stamm (EES 260)

lõust, gen. lôusta 'Grimasse, Fratze' keine Verbindung zu anderen osfi. Sprachen (EES 260) lõvi, gen. lõvi 'Löwe' < nd. louwe, lauwe, lowe, lewe 'Löwe' (EES 261)

mõhk, gen. mõha mõhu 'Ausbauchung, Ausbuchtung' Etymologie unsicher (EES 291)

mõhk, gen. mõhe 'Backtrog' ? gleicher Stamm wie est. mõhk, gen. mõha mõhu (s.o.) (EES 291)

mõhn, gen. mõhna 'Kuppe, Schwiele' Etymologie und Verbindung zu anderen osfi. Sprachen unsicher (SSA II: 194; EES 291)

mõigas 'Wulst' Etymologie unsicher (EES 291)

mõlk, gen. mõlgi 'Beule' keine Zusammenstellung mit anderen osfi. Sprachen (EES 292)

mõlkuda 'gedanklich in Bewegung sein' keine Zusammenstellung mit anderen osfi. Sprachen (EES 292) 
mõlu 'Totholz, das in einen Schaden bei lebendigem Holz eingewachsen ist' keine Zusammenstellung mit anderen osfi. Sprachen (EES 292)

mõõl, gen. mõõla 'Nelkenwurz (Geum)' keine Zusammenstellung mit anderen osfi. Sprachen (EES 294)

mõra 'Sprung, Riss' keine Zusammenstellung mit anderen osfi. Sprachen (EES 292)

mõrtsukas 'Mörder' ? < russ. мурзак; keine Zusammenstellung mit anderen osfi. Sprachen (EES 293), vgl. auch BLOKLAND (2009 174f.)

mõru 'bitter' keine Zusammenstellung mit anderen osfi. Sprachen (EES 293)

mõrv, gen. mõrva 'Mord' künstlich gebildeter Stamm (EES 293)

mõsta (dial.) 'waschen' keine osfi. Entsprechungen (EES 293)

mõtiskleda '(nach)sinnen, grübeln, meditieren' < fi. mietiskellä 'nachdenken, reflektieren, grübeln, meditieren' (EES 293)

nõksuda 'knacken, zusammenzucken, einen Ruck tun' keine Zusammenstellung mit anderen osfi. Sprachen (EES 324)

nõme, gen. nõmeda 'unwissend, laienhaft; schwachsinnig, dumm' künstlich gebildeter Stamm (EES 325)

nõnda 'so, derart' Etymologie unsicher (EES 325)

nõnge 'eigensinnig' künstlich gebildeter Stamm (EES 325)

õevane 'ausgezeichnet' Etymologie unsicher (EES 624)

õgida 'fressen, schlingen' Zusammenstellung mit anderen osfi. Sprachen unsicher (EES 624)

õis in Verbindung oisvesi 'Wund- oder Blasensekret' ? < balt., keine Zusammenstellung mit anderen osfi. Sprachen (EES 625)

õli, gen. õli 'Öl' < nd. olie, oley id. (EES 626)

õlp, gen. õlba 'Kleiderbügel' Zusammenstellung mit anderen osfi. Sprachen unsicher (SSA I: 214f.); künstlich gebildet (EES 626)

õõtssoo 'Schwingmoor' Zusammenstellung mit anderen osfi. Sprachen unsicher (SSA I: 160)

õõtsuda 'schaukeln, schwingen, wallen, wogen' Etymologie unsicher (SSA I: 160; EES 628)

õõv, gen. õova 'seltsame Angst' künstlich gebildeter Stamm (EES 628)

õpatada 'hüpfen, hopsen' keine Zusammenstellung mit anderen osfi. Sprachen (EES 627)

õriseda 'brummen, knurren' keine Zusammenstellung mit anderen osfi. Sprachen (EES 627) õrritada 'reizen, necken, hänseln' keine Zusammenstellung mit anderen osfi. Sprachen (EES 627) 
õssitada 'aufhetzen, aufwiegeln, hetzen, antreiben' keine Zusammenstellung mit anderen osfi. Sprachen (EES 627)

õu, gen. õue 'Hof' Etymologie unsicher, laut EES (627) ural., laut SSA (II: 277) < dt.

ouhka 'Äther' keine Zusammenstellung mit anderen osfi. Sprachen (EES 628)

oun, gen. õuna 'Apfel (Malus)' Etymologie unsicher, Zusammenstellung mit anderen osfi. Sprachen unsicher (SSA II: 265; EES 628)

plõmmida 'hämmern, klopfen' keine Zusammenstellung mit anderen osfi. Sprachen (EES 377) plõnnida 'kurz ein leises Geräusch (wie bei einer Saite) hören lassen, hämmern' keine Zusammenstellung mit anderen osfi. Sprachen (EES 377)

põis, gen. põie 'Blase' keine Zusammenstellung mit anderen osfi. Sprachen (EES 399)

põll, gen. põlle 'Schürze' unsichere Etymologie (EES 399)

põnev 'gespannt; fesselnd, spannend' wohl Variante vom gleichen Stamm wie est. pinev 'gespannt, gezwungen', dort keine Zusammenstellung mit anderen osfi. Sprachen (EES 371, 400) põngerjas '(kleines) Kind' ? unregelmäßige Variante von Stamm jõnger-, keine Zusammenstellung mit anderen osfi. Sprachen (EES 400)

põnn, gen. põnni '(kleines) Kind' keine Zusammenstellung mit anderen osfi. Sprachen (EES 400)

põnnata (ugs.) 'sich fürchten' keine Zusammenstellung mit anderen osfi. Sprachen (EES 400)

põnt, gen. põnda 'kleiner Hügel, höchste trockene Stelle' keine Zusammenstellung mit anderen osfi. Sprachen (EES 400)

põntsuda 'durch Schlag oder Fall ein Geräusch verursachen' keine Zusammenstellung mit anderen osfi. Sprachen (EES 400)

põrgu 'Hölle' unsichere Etymologie, mögliche Ableitung vom Wort est. pergel 'Teufel (Schimpfwort)' oder < balt. oder < germ. (EES 400), in SSA (II: 340) wird für est. pergel ? < fi. erwogen

põrpida 'erstaunen, verblüffen' künstlich gebildeter Stamm (EES 401)

prõmatada 'donnern, krachen, knallen' keine Zusammenstellung mit anderen osfi. Sprachen (EES 387)

rõhk, gen. rõhu 'Druck; Betonung' Etymologie unsicher (SSA III: 59; EES 440)

rõhuda 'drücken, pressen; unterdrücken; bedrücken' Etymologie unsicher, z.B. ? gleicher Stamm wie fi. rouhia 'schroten, zerstampfen, raspeln; misshandeln, ramponieren' (EES 441), wird dort in SSA (III: 96) nicht behandelt

rõigas, gen. rõika 'Rettich' < nd. redich, redik 'Rettich' (EES 441) 
rõlge 'Perverser' künstlich gebildet (EES 441)

rõme 'Furche, Unebenheit' Zusammenstellung mit anderen osfi. Sprachen unsicher (EES 441) rõmm, gen. rõmmi 'Konzentrat schwerer oder erzhaltiger Minerale, die man durch Waschen aus Sand oder anderen Stücken bekommen kann' keine Zusammenstellung mit anderen osfi. Sprachen (EES 441f.)

rõõduda 'rasselnd atmen' < russ. pbıdámb 'schluchzen' (BLOKLAND 2009: 243)

rõõdata 'rasseln, hausieren' (dial.) < russ. pblzámb 'rülpsen' (BLOKLAND 2009: 243)

rõuged 'Pocken' Zusammenstellung mit anderen osfi. Sprachen unsicher (EES 442); vgl. auch SSA (III: 96f.), wo est. rõuged nicht genannt wird

sõir, gen. sõira 'Hausgemachter Käse mit Kümmel' < russ. cbıp 'Käse' (EES 493; BLOKLAND 2009: 269)

sõkal, gen. sõkla 'Spelze, Korn-, Getreidehülse' < lett., keine Zusammenstellung mit anderen osfi. Sprachen (EES 494)

sõõn, gen. sõ̃̃na 'Strich, Streifen (Schmutz); Wolkenstreifen' unsichere Etymologie (EES 496) sõõre, gen. sõ̃rde 'Kreis, Ring' Zusammenstellung mit anderen osfi. Sprachen unsicher (SSA III: 217f.; EES 497)

sõrnik 'Quarkbällchen' < russ. сырник 'Quarkpuffer' (EES 495); vgl. BLOKLAND (2009: 270)

tõhk, gen. tõhu 'Iltis (Musteloa putorius)' ? wohl unregelmäßige Variante von est. tuhkur id. (EES 560)

tõik, gen. tõiga 'Tatsache, Faktum' künstlich gebildeter Stamm (EES 561)

tõld, gen. tõlla 'Kutsche' Etymologie unklar, laut EES (561) zu fi. telta 'Baldachin, der während der Trauung über das Brautpaar gehalten wird', in SSA (III: 282) werden fi. und est. nicht zusammengestellt, jedoch auf gleiches Ausgangswort (< skand.) zurückgeführt.

tõlk, gen. tõlgi 'Dolmetscher' < russ. толк, keine Zusammenstellung mit anderen osfi. Sprachen (EES 561); vgl. auch BLOKLAND (2009: 282)

tõlkjas 'Zackenschötchen (Bunias)' Entsprechung zu anderen osfi. Sprachen unsicher (EES $561)$

tõnnike 'Betonie (Betonica)' < dt. Betonie; keine Zusammenstellung mit anderen osfi. Sprachen (EES 562)

tõtata 'eilen, hasten' Stamm unbekannter Herkunft (EES 563)

tõug, gen. tõu 'Rasse, Gattung' Etymologie unsicher (EES 564)

tõugjas 'Rapfen (Aspius aspius)', unklare Etymologie, Zusammenstellung mit anderen osfi. Sprachen unsicher (SSA III: 315; EES 564) 
võdiseda 'beben, pendeln, schwingen' keine Zusammenstellung mit anderen osfi. Sprachen (EES 614)

võhi- in võhivõõras 'wildfremd' Etymologie unsicher (EES 614)

võigas, gen. võika 'befremdlich, seltsam, abscheulich' Zusammenstellung mit anderen osfi. Sprachen unsicher (EES 615)

võldas 'Groppe (Cottus gobio)' Herkunft unbekannt (EES 616)

võll, gen. võlli 'Welle (Maschine)' < nd. welle 'Welle, Walze; Zylinder' (EES 616)

võllas 'Galgen' Herkunft unbekannt (EES 616)

võlv, gen. võlvi 'Gewölbe, Wölbung, Bogen' < nd. welve id. (EES 616)

võnks, gen. võnksu 'Ruck, kurze plötzliche Bewegung' keine Zusammenstellung mit anderen osfi. Sprachen (EES 616)

võntsuda 'schlagen' keine Zusammenstellung mit anderen osfi. Sprachen (EES 617)

võpsik 'Schössling, Spross, Gestrüpp' ? unregelmäßige Variante von Stamm est. võsa 'Gebüsch' (EES 617)

võrada 'surren, schwirren, schnurren' keine Zusammenstellung mit anderen osfi. Sprachen (EES 617)

võrel, gen. võrgli 'Gitter' Zusammenstellung mit anderen osfi. Sprachen unsicher (SSA III: 427)

võru 'Reifen, Ring' Zusammenstellung mit anderen osfi. Sprachen unsicher (SSA III: 427; EES 617)

võst, gen. võstu 'Annahme, Empfang' künstlich gebildet (EES 617)

\section{WOTISCH}

bõ Partikel, Etymologie unbekannt

bõrisa 'flennen, summen (Insekten), rattern' Etymologie unbekannt

brõizguttaa 'spritzen, zerstäuben' Etymologie unbekannt

brõss 'starker Wind, Brise' Etymologie unbekannt

brõõts 'kusch' Etymologie unbekannt

dõ 'und, sowie, aber, doch' Etymologie unbekannt

glõõza, gen. glõozaa 'Erdklumpen' ? < гльба 'Abbruch, Klumpen'

gõndia 'umherstreichen, schlendern, bummeln, faulenzen' Etymologie unbekannt grõba 'Pilz; Ballen' ? < russ. гриб 'Pilz'

grõšsiaG, grõzia, grõzziaG 'nagen, beißen' ? < russ. гpblзmb 'etwas anknabbern, annagen' grõžovnikka 'Stachelbeere' ? < russ. крыжовник 'Stachelbeere' 
grõõza, gen. grõ̃zaa 'Bruch' ? < russ. грыљжа 'Bruch, Hernie'

hõllõassi 'schlaff, schlapp, welk' Etymologie unbekannt

hõmõssua 'schimmeln' Tsv.

hõrkkikõrva 'Spitzohr, mit gespitzten Ohren (Hund)' Etymologie unbekannt

$\chi \tilde{\mathbf{o}}$ Bezeichnung des russ. Buchstaben $\chi$

klõkki 'Schicht' Etymologie unbekannt, ? < russ. клькк 'Hauer, Reißzahn'

kõaD 'Innenorgane von (Haus)tieren' Etymologie unbekannt

kõblaz 'Schlittenspeiche' Tsv.

kõhata 'krank machen, mit einer Krankheit befallen' Etymologie unbekannt

kõitama kõitõmõ, gen. kõitamaa, kõitõmaa 'Querholz der Tischbeine' Etymologie unbekannt

kõlpa, gen. kõlvaa 'passend, angebracht' Tsv.

kõlsta-kangaz 'flacher, leinener (Woll)stoff' Etymologie unbekannt

kõnsnii 'jederzeit, wann immer' Etymologie unbekannt

kõpikõikõllaajun 'jeder beliebige, alles Mögliche' Etymologie' unbekannt

kõpõstua 'mit Pech bedecken' Etymologie unbekannt

kõri, gen. $-i$ 'Gurgel, Kehle' Tsv.

kõrpi 'Wüste' Etymologie unsicher, in SSA (I: 405) und EES (201) nicht behandelt, laut VKS (541) ebenso ?

kõrrallin 'Fronarbeiter' Etymologie unbekannt

kõrvõttaa 'verbrennen (bei Brennesseln) ' Etymologie unbekannt

kõrõ, gen. kõrõ õ 'Kiel' Etymologie unbekannt

kõõ 'wie' Etymologie unbekannt

krõl'ttsa 'Windfang (mit Treppe), Außentreppe' ? < russ. крыльцьо 'Aufgang, Vortreppe'

krõõpata 'knirschen' Etymologie unbekannt

krõõsa -ssa, gen. krõõzaa 'Ratte' ? < kpbíca 'Ratte'

krõõška, gen. krõõžgaa 'Deckel' ? vgl. russ. крышка 'Deckel, Kappe'

krõõǒžmarja 'Krähenbeere' Etymologie unbekannt

Iõhna, gen. lõhnaa 'Windhauch; weich, warm, sanft (Wind)' Tsv.

Iõntata 'schleppend gehen' Etymologie unbekannt, in VKS (657) ebenso ?

lõugata 'betteln, flehen' Etymologie unbekannt

lõuhua 'gerührt werden, weich werden' Etymologie unbekannt

lõuka in tšivilõuka 'Meeresmuschel' Etymologie unbekannt

Iõutto, gen. lõutoo 'kleines Grundstück oder Feldstück oder -streifen' Etymologie unbekannt 
lõvata '(im Kreis) gehen, umherstreifen, hasten' Etymologie unbekannt lõõkku, gen. lõ̃kuu 'Schaukel, Wiege' Etymologie unbekannt Iõõz Refrainelement in Wiegenliedern, Etymologie unbekannt Iõõzata 'schlendern, bummeln' Tsv.

lõõža, gen. lõõzaa 'Ski, Schneeschuh' ? < russ. льжа 'Ski, Schneeschuh' Iõõttsussa 'schaukeln, schwingen, schweben' Tsv. mõila, gen. mõilaa 'Seife' ? < russ. мьлло 'Seife' mõlkõa 'fein, dünn' ? < russ. мелкий 'klein, flach, fein' mõlta, gen. mõllaa 'Kreide' ? < russ. мел 'Kreide' mõnõll-vootta 'manchmal' Tsv. mõrissa 'brummen, knurren' Etymologie unbekannt mõrttsu, gen. mõrtsuu 'Heusack' Etymologie unbekannt nõdrõma 'Bogensehne' Etymologie unbekannt nõh Interjektion, Etymologie unbekannt nõikku 'Bogen der Wiege; Brunnenwinde' Etymologie unbekannt nõjahtaa 'lehnen, sich anlehnen, sich stützen' Etymologie unbekannt nõkata 'nicken' Etymologie unbekannt nõrisa 'rinnen, sickern' Etymologie unbekannt nõrkata 'abtropfen, nach und nach gießen' Etymologie unbekannt nõru, gen. nõruu 'Netzkasten, -mulde' Etymologie unbekannt nõssõmuz 'Last, Bürde' Tsv. nõusu, gen. по̃иzии 'Einkommen, Einnahme' Tsv. põippõ, gen. põipõ̃ 'Küken' Etymologie unbekannt põkõrtõlla 'durch Schnee waten' Etymologie unbekannt põldikko 'mit Nesseln bedeckte Fläche' Tsv. põrgata 'flitzen, rennen' Tsv. põrku 'Hölle, Schmähung' Tsv., < est. (SSA II: 340) põtkõz, gen. põtkõ õ 'Wiesenkerbel' Etymologie unbekannt põukku, gen. põukuu 'durchweichte Wäsche' Etymologie unbekannt prõzgata 'spritzen, bespritzen' ? < russ. брызгать 'abspritzen, aufspritzen, -sprühen' prõõgata 'aufspringen, hüpfen' Tsv. prõõzguttaa 'sich belauben' Etymologie unbekannt rõbakka 'Fischer' ? < russ. pыбак 'Fischer, Angler' 
rõhloi 'locker, bröcklig, mürbe' Tsv.

rõhu, gen. rõhuu 'Korn, Krume, Brocken, Körnchen' Etymologie unbekannt rõju 'Abfall, Plunder' Etymologie unbekannt

rõggahtaassa 'grunzen' Tsv.

rõngõssaa 'Kreis, Ring schließen' Tsv.

rõssua 'wegschmelzen' Etymologie unbekannt

rõuna, gen. rõunaa 'schlau, listig; hinterlistig' Etymologie unbekannt

rõuta, gen. rõvvaa 'Bodeneis, dünne Eisschicht' Etymologie unbekannt

rõõgata 'heiser sprechen, schnauben (Pferde), keuchen' Tsv.

rõõmu, gen. rо̃отии 'unsauberer, unordentlicher Mensch' Tsv.

smõtška, gen. smõdžgaa 'Geigenbogen' Tsv.

sõisoa 'bezahlen, wert sein' Etymologie unbekannt

sõittõlu 'Zank, Streit' Etymologie unbekannt

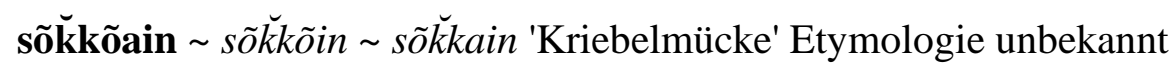

sõlkõuz 'Schnürverschluss' Etymologie unbekannt

sõputtaa 'schütten, streuen; tröpfeln' ? < russ. cblnamb 'schütten, streuen'

sõpõuta 'anziehen, kleiden' Etymologie unbekannt

sõra 'Zahn (einer Sichel)' Etymologie unbekannt

sõrajõška 'Täubling' Tsv.

sõroi 'feucht, zäh; teigig (Brot)' Tsv.

sõromatnoi 'getrocknetes, ungegerbtes Leder' Tsv.

sõrtua 'gerinnen (Milch) ' Tsv.

sõrva 'Rand, Kante' Tsv.

sõttua 'sterben, erlöschen' Etymologie unbekannt

sõvalliizõD 'Menstruation' Etymologie unbekannt

sõvõta 'kleiden, sich ankleiden' Etymologie unbekannt

sõõra, gen. sõ̃oraa 'Käse (aus dem Laden)' Tsv.

stõra, gen. stõraa 'Penis (Säugetier)' Etymologie unbekannt

šlõkka, gen. šlõǩkaa 'Kopfbedeckung der verheirateten Frau' ? vgl. russ. шльк 'Kopfbedeckung einer verheirateten Frau' (VKS 1234)

šõpõtäi 'Kleiderlaus' Etymologie unbekannt

tõ 'ja, doch, denn, dennoch' Etymologie unbekannt

tõkkua '(herunter-, weg)fallen, stürzen' Etymologie unbekannt 
tõkva, gen. tõkvaa 'Kürbis' ? < russ. mblквa 'Kürbis'

tõnttu, gen. tõntuu 'Strömling' Etymologie unbekannt

tõrguz, gen. tõrkuu 'Quelle' Etymologie unbekannt

tõrkkia 'stechen, stecken' Etymologie unbekannt

tõtõlla 'gehorchen, gehorsam sein' Etymologie unbekannt

võdna, gen. võdnaa 'Lamm' Etymologie unbekannt

võdra, gen. võdraa 'Fischotter, Elch' ? < russ. вылдра 'Fischotter'

võkroikka 'Schnitt' ? < russ. вьлкройка 'Schnitt, Zuschnitt'

võlgõssaa 'borgen, entleihen' Tsv.

võõdumka 'Ausgedachtes' Tsv.

võõgodnoi 'nützlich, rentabel, vorteilhaft' Tsv.

võõno, gen. võ̃̃noo 'langsam; friedlich, ruhig, missmutig; gleichgültig' Etymologie unbekannt võõrutška 'Lade, Schublade (des Ladentischs)' Tsv.

võõšittaa 'ausnähen, sticken' Etymologie unbekannt

võõveska 'Schild' Tsv.

võõvolotška 'Zausen, Zerren' ? < russ. выволочка 'Abkanzelung, Rüge'

võra, gen. võraa 'Opfer, Opfergabe' Etymologie unbekannt

võrka, gen. võrgaa 'Zipfel (des Rocks, Sarafans, Hemdes); Gürtel, gekauftes Tuch' Etymologie unbekannt

võrrata 'vorwerfen, herumnörgeln' Etymologie unbekannt

võrtna 'Ausläufer (des Hakenpflugs)' Etymologie unbekannt

võrvanattu 'Stümper, Pfuscher' Etymologie unbekannt

võttauta 'erscheinen, entstehen' Etymologie unbekannt

võvva, gen. - $a a$ 'älterer Bruder' Etymologie unbekannt

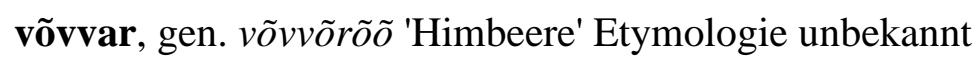

õhkussa 'glühen, glimmen' Tsv.

õhsunuz 'Verstoß, Irrtum' Etymologie unbekannt

õimõD 'Gräten' Etymologie unbekannt

õisa, gen. õizaa 'Segge, Riedgras' Etymologie unbekannt

ojjua 'schwimmen, bewegen' Etymologie unbekannt, in SSA (III: 368) und EES (576f.) nicht zu fi. uida 'schwimmen' bzw. est. ujuda id. gestellt

õjõla 'dünn, benutzt (Kleidung, Leder)' Etymologie unbekannt

õlõmatoo -oitoo -õtoo 'arm, dürftig' Etymologie unbekannt 
õlõmiin 'Besuch bei den Eltern Zuhause' Etymologie unbekannt

õlõvõin 'reich' Etymologie unbekannt

õmaa-äd'd'ä 'Großvater oder Schwiegervater (mit dem man zusammenlebt)' Etymologie unbekannt

õmaa-ämmä 'Großmutter oder Schwiegermutter (mit der man zusammenlebt)' Etymologie unbekannt

õmõ 'Schimmel; Schlamm (nach dem Schmelzen des Schnees); Schmutzstreifen' Etymologie unbekannt

õõ Interjektion, Etymologie unbekannt

õõlama '(Holz)höhlung' Etymologie unbekannt

$\tilde{\mathbf{o}} \tilde{\mathbf{o}}^{2}, \tilde{\mathbf{o}} \tilde{\mathbf{o}}^{2}$ verneinter Imperativ (ugs.), Etymologie unbekannt

õraz 'junge aufgegangene Saat von Getreidepflanzen' Etymologie unbekannt

õrauta 'sauer werden (Milch)' Etymologie unbekannt

orisa 'brummen, knurren' Etymologie unbekannt

õrjua 'schwanken, wanken' Etymologie unbekannt

õtsata 'fortsetzen, weiterknüpfen' Tsv.

ouna 'Apfel' Etymologie unsicher, Zusammenstellung mit anderen osfi. Sprachen unsicher (SSA II: 265; EES 628)

õvvi 'Innenhof; Viehstall (des Kolchos)' , laut EES (627) ural., laut SSA (II: 277) < dt.

\section{LIVISCH}

bõ̃mbandõks 'Anschwellung, Geschwulst, Ödem' Etymologie unbekannt

bõ'ṇdž̃̃ 'flennen, wimmern; rattern, summen' Etymologie unsicher (LW 22)

berìkšâ 'bäken' Etymologie unsicher (LW 22)

bõuv 'Gebäude' < mnd. būwen (LW 22)

begzàs 'in einem Haufen zusammengekrümmt' Etymologie unsicher (LW 22)

brõuvõ 'brauen' < mnd. brūwen (LW 22)

dõ'ŗ̌žõ 'schlagen; erschüttert werden; zittern' Etymologie unsicher (vgl. SSA III: 356; LW 37)

glõ'bžõ 'klappern' keine Zusammenstellung mit anderen osfi. Belegen (vgl. LW 59)

gezà 'Regenwolke, große Wolke' keine Etymologie (LW 56)

jẹ'ư̌vâ 'schleifen' keine gesicherte Etymologie (LW 90f.)

klõkšõ 'klatschen, knallen lassen; schlucken; schalten; (Vertrag) schließen' keine Etymologie (vgl. auch LW 139) 
klẹñtšô 'langsam gehen; schlendern' keine gesicherte Etymologie (LW139)

klō̃rõ 'kreisen, Kreise ziehen' laut LW (139) lautmetaphorisch

knẹ'bbil' 'Lehne, Stuhllehne' laut LW (142) „deskr. Umbildungen“

kȭidaz 'Weberkamm' keine Etymologie (LW 118)

kõlā-kõlā 'schlängelnd, gewunden' Etymologie unbekannt

kȭlbantõ 'bauen, anwenden' Etymologie unbekannt

kȭlbatõks 'Begriff' Etymologie unbekannt

kỗlim '(Birken)saft' < kẹùv 'Birke' + lēm 'Saft' (LW 121)

kõm 'Ausflucht, Ausrede' Etymologie unbekannt

kẹ̄m 'Verstand; Knorpelchen' keine Etymologie (LW 118f.)

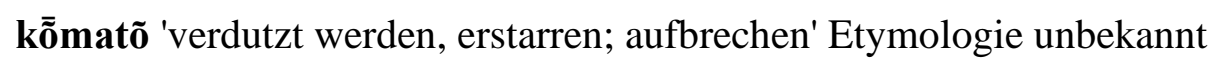

kõ̃nka 'Düne, Hügel' Etymologie unsicher (vgl. LW 121; SSA I: 464; EES 214)

kệnâ 'sich rekeln' keine Zusammenstellung mit anderen osfi. Belegen (vgl. LW 121)

kõps 'Hase' keine Etymologie (LW 119)

kõ'r 'Ring, Kreis, Runde' Etymologie unsicher (LW 119; SSA I: 354; EES 141)

kè 'r 'Schafgarbe' Etymologie unsicher (LW 119)

k $\overline{\tilde{o}}$ rdikšõ 'schelfern; hart werden' Etymologie unbekannt

kō̃rdiṇtõ 'vervielfältigen, multiplizieren' Etymologie unbekannt

kō̄rtan 'Umschlagetuch' ? > germ. (LW 122); von LÄGLOS (II: 130) abgelehnt, liv. dort nicht behandelt

kõrtõ 'verdauen' Etymologie unbekannt

kõ'ṛ 'Schachtelhalm' von SSA (I: 406) und EES (202) nicht zu fi. korsi Halm; SchlammSchachtelhalm' bzw. est. kõrs 'Halm' gestellt

kevìstâ 'durchreiben schleifen' Etymologie unsicher (LW 120)

keviviń (eine Art Pilz) keine Etymologie (LW 120)

krõdā 'Höhle, Loch' Etymologie unbekannt

krẹntsŝl 'zusammengeschrumpftes Wesen (Schimpfwort)' keine Etymologie (LW 154)

krẹ̄G 'Birkenmaser' keine Etymologie (LW 154)

lõbāz 'geräumig, weit' Etymologie unbekannt

lẹ̀ida 'hölzerne Schüssel' keine Etymologie (vgl. LW 189)

lõ̃a 'Holzteller; Holzschale zum Einsalzen von Fisch' Etymologie unsicher (vgl. LW 190)

lõ̃iga 'Überschuss, Überfluss' Etymologie unsicher (vgl. SSA II: 72; EES 239)

lỗkald 'halboffen' Etymologie unbekannt 
lõks 'Falle' Etymologie unbekannt

lõpsnā 'freundlich, gefällig' Etymologie unbekannt

lẹpsù-kalà 'Steinsauger' Etymologie unsicher (vgl. LW 189f.)

leg' u 'regelrecht zusammengeformter Haufen, den man mit einem Mal fassen kann' Etymologie unsicher (vgl. LW 190, SSA II: 67)

lẹ $\mathbf{u} \breve{p}-\mathbf{p} \overline{\mathbf{u}}$ 'Stange im Webstuhle, die das Gewebe stramm hält' Etymologie unsicher (vgl. LW 190; EES 260)

lõuž 'Kopfschuppen; aus Kiefernrinde gemachtes Brettchen am Netze' keine Etymologie (LW 190)

lõ'vvõ 'wünschen; bestimmen; auswählen' Etymologie unbekannt

mȭita 'sonst, umsonst; anders' Etymologie unbekannt

mõllõ 'wühlen; zausen' Etymologie unsicher (vgl. LW 221)

mõltsi 'grün' Etymologie unsicher (LW 221; EES 280)

mõrā 'Surren' Etymologie unbekannt

mõrtš 'Doppelkinn, Wampe; Kapuze' Etymologie ungesichert (LW 221; est. mõrts in EES nicht behandelt)

nō̃ Interjektion, Etymologie unbekannt

nõrī 'steif, starr, unbiegsam' keine Etymologie (vgl. LW 245)

nõrīkšõ 'murren, näseln' keine Etymologie (vgl. LW 245)

nõ'rrõ 'tauchen' Etymologie unbekannt

nõ'ṛ 'Windung', LW: 'knotige Stelle im Garn oder Strick, Kringel im Garn oder Eisendraht' keine Etymologie (vgl. LW 245)

nõ'ṛṛõ 'kauen, nagen' keine Etymologie (vgl. LW 245)

nõtk 'Drehung' Etymologie unbekannt

nẹtkômôz 'Feder; Gelenk; Silbe' laut LW (246) zu est. nõtke 'Pflock, an den etwas gehängt wird', welches in EES nicht behandelt wird

nệtkìńt't'tô 'beugen' ? Neologismus (LW 246)

$\overline{\tilde{\mathbf{o}}}$ 'gõ 'schmerzen' Bedeutung sekundär (vgl. LW 52f.)

õlastõ 'zechen, schlemmen; jubeln, jauchzen' Etymologie unbekannt

ó'Igõ 'funkeln, flimmern, glänzen' Etymologie unbekannt

êlk̆kaz 'regelmäßig gewachsenes, leicht spaltbares (z.B. Holz)'unsichere Etymologie (LW 53)

$\tilde{\mathbf{o}}^{\prime} \mathbf{m}$ 'Verstand' Etymologie ungesichert (LW 51 ?

õpkāz 'Eber' keine Etymologie (vgl. LW 51) 
ẹpslaǩkkà 'Ärmelaufschlag; [...]' > mnd. upslach (LW 51)

õ̃rbimi 'Abnutzung, Verschleiß' Etymologie unbekannt

õ'ŗštõ 'hetzen, reizen' keine Zusammenstellung mit anderen osfi. Belegen (vgl. LW 51; SSA I: 190; EES 627)

õvā 'Strömung' keine gesicherte Etymologie (vgl. LW 51f., in EES nicht belegt)

plõukštõ 'knallen, applaudieren' < lt. (LW 302), keine Zusammenstellung mit anderen osfi.

Belegen

*pèör 'drehen, wenden' SjW (LW 284)

põp 'Flachssschäbe' laut LW (283) zu est. põpe, welches in EES nicht behandelt wird

põrāndõks 'Feuer' LW auch: 'Scheiterhaufen' ? zu liv. põ̃rand (LW 283f.)

põ'rrõ 'verfallen, zerfallen' Etymologie unbekannt

pẹ̄gâ 'bersten; springen' ungesicherte Etymologie (LW 284)

põzāg 'Staubkorn, Stäubchen' Etymologie ungesichert (LW 284)

rō̃msõ 'vermengt, durcheinander' Etymologie unbekannt

reömôst 'rauben, plündern' $\mathrm{SjW}$ (LW 335)

rõ'v 'Rasen' Etymologie ungesichert (vgl. LW 335; SSA III: 60)

skrõuv 'Schraube' < mnd. schruve 'Schraube' (LW 373)

sẹinastâ 'etwas anschimmeln' keine Etymologie (LW 360)

sõ̃ira 'Käse' < russ. (vgl. LW 360) ? < est (EES 493)

*seìt 'überflüssig' SjW (LW 360)

sõ'ņ̦țõ 'verleumden, schmähen' Etymologie unbekannt

seöD 'Speise; Lockspeise, Köder' SjW (LW 361)

sõ'v 'Sommer' ? zu est. suvi id., laut LW „Tendenzanalogie“ (LW 361), in SSA (III: 228) wird liv. Variante nicht behandelt

sõvā 'Stock, Stab; Ton' Etymologie unbekannt

sõ' vlõ 'jucken' Etymologie unbekannt

spõrkšlõ 'plärren' Etymologie unbekannt

špõrkstõ 'sausen; summen' LW auch: 'schwirren' ? lett. (LW 397)

sprõukšlõ 'sich räuspern; schnaupen, prusten' < lett. (LW 380)

stõukstõ 'schlagen, pulsieren' Etymologie unbekannt

tỗla 'Wäschebleuel' LW auch: 'Klöpfel, Schlegel, Keule' laut LW (416) zu est. tõlv 'Keule'; liv. < est. (EES 561)

tỗlatõ 'klopfen, hauen; rollen, mangeln' Etymologie unbekannt 
tõldõz 'Meilenstein' Etymologie unbekannt

tỗlpa 'Pfosten, Säule' Etymologie ungesichert (LW 416)

tõmblõ 'sich raufen, sich streiten' Etymologie unbekannt

tõmšlõ 'reißen, ziehen; spannen' Etymologie unbekannt

tõrg 'Markt, Jahrmarkt' laut LW (415) zu est. turg 'Markt', in SSA (III: 335) und EES (556)

jedoch nicht dort behandelt

*tegrG 'zanken, maulen' SjW (LW 415)

*tệrst 'in den Kleetenkasten füllen' SjW (LW 415)

tõ'udõd 'Salbe-Pl' Etymologie unbekannt

tõ'udõz 'Brunneneinfassung' LW auch: 'Seitenholz, Bodenrand; Kornkasten' Etymologie ungesichert (LW 415f.)

tõuk 'Menge, Summe' Etymologie unbekannt

võ'ilõ 'flattern; winken, schwingen' Etymologie ungesichert (LW 482)

vỗlastõ 'vorschweben' Etymologie ungesichert (LW 484)

vȭlatõ 'wüten, grassieren' Etymologie unbekannt

võṇdž̃̃ 'summen' Etymologie unbekannt

vegràndôks 'Geräusch' Etymologie unsicher (LW 482f.)

võse'm või se u'm 'oder ist es' Form von schneller Aussprache (LELS 375)

võttõks 'Zweck; Plan' Etymologie unbekannt

zèöks 'hüpfen, tanzen' SjW (LW 399)

\section{SÜDESTNISCH}

hõdras, gen. .hõtra 'zerbrechlich, brüchig, zart' Etymologie unbekannt

hõdśo 'Struma, Kropf (bei Vögeln)' Etymologie unbekannt

hõginallaq 'gierig, gefräßig' Etymologie unbekannt

hõi(q) Begrüßung, Etymologie unbekannt

hõikma, .hõikuq, 'rufen' Etymologie unbekannt, ? zu est. hõigata 'rufen'

.hõiskama, hõisadaq 'jubeln' Etymologie unbekannt, wohl zu est. hõissa (s.o), welches < dt.

hõkatõ|(I)lõma, -llaq 'gackern, kichern' Etymologie unbekannt

hõkõrda|ma, $-q$ 'lachen, kichern' Etymologie unbekannt

hõla 'Sehnsucht, Wunsch; Traum' ? zu est. hõllandus (s.o.), etymologisch nicht belegt

hõlgas, gen. .hõlka 'leicht zu spalten' Etymologie unbekannt

hõl'ova 'Ätherspiritus' Etymologie unbekannt 
hõlst́, gen. hõlsti 'Kittel' Zusammenstellung mit anderen osfi. Sprachen nicht gesichert (SSA I: 192)

hõlõstik 'lichter Wald' Etymologie unbekannt

.hõpśma, .hõpsiq 'löffeln' Etymologie unbekannt

hõrga|tś, gen. - $d s i$ 'Fessel (Pferd)' Etymologie unbekannt

hõrgulõ 'locker, bauschig' Etymologie unbekannt

hõrgu|tama, -taq 'locker machen, leicht ausbreiten, schütteln, rütteln' Etymologie unbekannt

hõril|aq, gen. -(i)dõ 'Orgel' Etymologie unbekannt

.hõrksa 'zerbrechlich, brüchig, zart' Etymologie unbekannt

hõrn, gen. .hõrna 'Bachforelle' Etymologie unklar, wohl zu est. hõrnas id. (s.o.)

hõrrõ 'undicht, spärlich, dünn' (zu est. hõre s.o.)

.hõrssama, horrsadaq 'schimpfen, grunzen, quieken' Etymologie unbekannt

hõrts, gen. hõrdso 'Wasserloch, sumpfige Stelle' Etymologie unbekannt

.hõrtsama, hõrdsadaq '(herum)tollen; schelten' Etymologie unbekannt

hõr|u, gen. - $u$ 'starke Kälte; Trauerschleier' Etymologie unbekannt

hõrõ|tama, -taq 'rot sein' Etymologie unbekannt

hõ|til, gen. -dila 'rein; Kartoffelknospe' Etymologie unbekannt

hõõda|k, gen. - $g u$ 'fremd, unverständlich (Sprache)' Etymologie unbekannt

hõõlas, gen. hõ ỗla 'Reihe'

jõbis|õma, -taq 'wiedersprechen, murren; schwatzen' Etymologie unbekannt

jõhi|k, gen. -gu 'Rohling, Grobian' Etymologie unbekannt

jõhka|tama, -taq 'umherstreichen; ununterbrochen nagen oder essen; vor und zurück bewegen'

Etymologie unbekannt

.jõhḿma, .jõhmiq 'gierig essen' Etymologie unbekannt

.jõkśma, jõ ksiq 'schlucken' Etymologie unbekannt

jõlahõ|(I)lõma, -llaq 'neugierig herumstarren' Etymologie unbekannt

jõlduń, gen. - $i$ 'kleiner Junge' Etymologie unbekannt

jõlli|tama, -taq 'Augen aufreißen' Etymologie unklar, s.o. est. jõllis 'hervorgequellen, aufgerissen (Augen)'

jõlvõh(t)u|ma, $-d a q$ 'weich, kraftlos werden' Etymologie unbekannt

jõlvõs, gen. - $(s) \tilde{o}$ 'ungehorsamer Mensch; Rumtreiber' Etymologie unbekannt

jõ|pin, gen. -bina 'Einwand, Murren, Gebrumm; Geschwätz' Etymologie unbekannt

.jõpśma, gen. .jõpsiq 'Geschlechtsverkehr haben' Etymologie unbekannt 
jõrga in jõrgapill' 'Akkordeon, Akkordeonspiel' Etymologie unbekannt .jõrmama, jõrmadaq 'weinen, schreien, brüllen' Etymologie unbekannt .jõrssama, jõrsadaq 'herumstreichen; sich herumtreiben; herumtollen' Etymologie unbekannt jõõrah|(t)uma, -tudaq 'vom Weg rutschen; schief gehen' Etymologie unbekannt

jõõsand́, gen. $-i$ 'grob; unstet; ungehorsamer Mensch, ungehorsames Tier' Etymologie unbekannt

jỡmḿ, gen. jõ õmmi 'Dickerchen; kleiner Junge' Etymologie unsicher, s.o. est. jõmm id.

jõmmõlda|ma, $-q$ 'verstauchen, verdrehen' Etymologie unbekannt jỡndõrda|ma, $-q$ 'vor und zurück gehen; schlendern' Etymologie unbekannt jõ $\mid$ |tin, -dina 'Schauer, Zittern, Beben' Etymologie unbekannt jyyrdma, jõ õrdaq 'vom Weg rutschen; schief gehen' Etymologie unbekannt (k)lõka|tama, -taq 'wackeln, klappern' Etymologie unbekannt .(k)lõkśma, .(k)lõksiq 'schlucken' Etymologie unbekannt klõpa|tama, -taq 'klappern' Etymologie unbekannt klõpa|tś, gen. - $d s i$ 'Knirps, kleines Kerlchen' Etymologie unbekannt klõsśs, gen. klõssi 'Magermilch, entrahmte Milch' ? zu est. lõss id.

klộmahk, gen. - $u$ 'Schluck; Krach' Etymologie unbekannt (k)lộmba 'Lahmer, Gehbehinderter' Etymologie unbekannt .klōmmóma, .klốmpiq 'löffeln, quasseln, schlürfen' Etymologie unbekannt klōnkś, gen. klynksi 'Schluck' Etymologie unbekannt kõbasta|ma, $-q$ 'schärfen' Etymologie unbekannt kõblas, gen. .kõpla 'Hacke' ? zu est. kõblas 'Hacke, Picke', estS. in EMS nicht belegt kõbli 'Meißel, „Messerlöffel““ (Form von Besteck)' Etymologie unbekannt kõ $\mid \mathbf{d s a}(\mathbf{h a}) \mathbf{m a}$, -tsaq 'säuseln, leise rauschen' Etymologie unbekannt

kõ|dśo, gen. -dśo 'kümmerliches Heu' Etymologie unbekannt kõdśo|tama, -taq 'langsam fahren' Etymologie unbekannt kõdõr, gen. kõdra 'Schote' Etymologie unbekannt kõhikõva 'sehr hart, fest, streng' Etymologie unbekannt kõhku|tama, -taq 'trödeln' Etymologie unbekannt kõhma 'fauler Mensch' Etymologie unbekannt kõhŕ, gen. kõhri 'Nacken, Genick' Etymologie unbekannt kõht, gen. kõha 'Kraft, Vermögen' Etymologie unbekannt kõhvi|tsõma, -tsaq -daq 'kratzen' Etymologie unbekannt 
.kõhvõlõma, kõhvõldaq 'unruhig hin und her rutschen; trödeln' Etymologie unbekannt

kõlgas, gen. .kõlka 'Kaffscheune' Etymologie unbekannt

kõlga|tś, gen. $-d s i$ 'Kuhglocke' Etymologie unbekannt

kõpi|tsõma, $-t s a q \sim-d a q$ 'peinlich genau arbeiten, Kleinarbeit machen' Etymologie unbekannt

.kõrdliganõ 'geschichtet' Etymologie unbekannt

kõrdmago 'Blättermagen, Teil des Vormagens von Wiederkäuern' Etymologie unbekannt

kõrgatś 'weißer Reizker' Etymologie unbekannt

kõrik 'Decke, wollenes Umschlagtuch' < russ., vgl. BLOKLAND (2009:146f.)

kõrru|tama, -taq 'Brot oder Piroggen machen; kräuseln (nähen)' Etymologie unbekannt

kõrrõ 'zart, schlank' Etymologie unbekannt

kõrtś, gen. kõrdsi 'Kneipe' < russ. s.o. est. kõrts 'Kneipe, Krug, Schenke'

.kõrtśni|k, gen. - gu 'Kneipenwirt' < russ. s.o. est. kõrtsmik id.

kõrõstik '(Eises)kälte' Etymologie unbekannt

kõsisõma 'säuseln, rauschen' Etymologie unbekannt

.kõsli 'taubes Korn' Etymologie unbekannt

kõs $\mid \mathbf{u}$, gen. - $u$ 'trockener (Getreide)staub, Abfall, Hülsen' Etymologie unbekannt

kõtsi Interjektion (um Tiere zu scheuchen) Etymologie unbekannt

kõur, gen. kõuri 'großer, krummer, astreicher Baum' Etymologie unbekannt

kõvasḱ, gen. - $i$ 'Schleifstein' Etymologie unbekannt

kõõbõnda|ma, $-q$ 'mühsam arbeiten' Etymologie unbekannt

kõõodśa 'Greis; lebensfähig' Etymologie unbekannt

kõõland', gen. $-i$ 'fauler Mensch' Etymologie unbekannt

kõõlasḱ, gen. $-i$ 'magerer Mensch, Stange' Etymologie unbekannt

kõõni|tsõma, -tsaq -daq 'Schwendland von vom Brennen übrig gebliebenem Holz reinigen'

Etymologie unbekannt

kõõri|k, gen. - $g u$ 'schief, scheel' Etymologie unbekannt

$\mathbf{k y d}(\mathbf{s}) \mathbf{i s} \mid \mathbf{o} \mathbf{m a}$, -taq 'flüstern, raunen' Etymologie unbekannt

kymmõlda|ma, - $q$ 'faulenzen, müßig sein' Etymologie unbekannt

kõnnń, gen. kõnnni 'Eigensinn, Trotz' Etymologie unbekannt

kynứ, gen. - $i$ '(Holz)Reifen unter dem Rock' Etymologie unbekannt

kõnõrus 'Kreuzbein; Teil des Hakenpflugs' Etymologie unbekannt

kõõọksa 'biegsam, zäh' Etymologie unbekannt

kõõómõlõma, kõomõldaq 'faulenzen' Etymologie unbekannt 
kõõvama, kõovvadaq 'lodern, brennen, glühen' Etymologie unbekannt

krõ|bu, gen. - $b u$ 'Krümchen, Staubkorn' Etymologie unbekannt

krõhv́, gen. krõhvi 'Eigensinn, Trotz' Etymologie unbekannt

krõhva 'magere böse Frau' Etymologie unbekannt, ? zu est. krõhva id.

k(r)õhvi|tsõma, -tsaq - daq 'kratzen, kraulen' Etymologie unbekannt

krõim, gen. krõima 'Eigensinn; Teil einer Mühle' Etymologie unbekannt

krõõbi|tsõma, -tsaq -daq 'schaben, radieren' Etymologie unbekannt

krõõdsa|tama, -taq '(mit einer stumpfen Säge) sägen' Etymologie unbekannt

.krỗl'ma, .krylliq 'trotzen, eigensinnig sein' Etymologie unbekannt

krymmõ 'heftig, hitzig, scharf, ungestüm' Etymologie unbekannt

.krộngli 'Kringel, Brezel' ? wohl nd., vgl. est. kringel id. (EES 183)

krọ̃nkś, gen. krợnksi 'Krümmung, Biegung' Etymologie unbekannt

krỗ |ńo, gen.-ńo 'Klepper' Etymologie unbekannt

krõ̃õgama, krõ̃oadaq 'röcheln' Etymologie unbekannt

krõō

kryyś, gen. krõõsi 'Angelgerät' Etymologie unbekannt

krõõottsa 'hübsch, nett' Etymologie unbekannt

lõbasḱ, gen. - $i$ 'Schwätzer' Etymologie unbekannt

lõ|bima, -piq 'fressen, schlingen' Etymologie unbekannt

lõhva 'Schwätzer' Etymologie unbekannt

lõigus, gen. - $(s) \tilde{o}$ Lied bei den Setus, Etymologie unbekannt

lõka|tama, -taq 'schwätzen, schwatzen' Etymologie unbekannt

Iõpõrda|ma, $-q$ 'schwatzen, schwätzen' Etymologie unbekannt

.lõrvama, lõrvadaq 'brüllend weinen' Etymologie unbekannt

lõssõ|tama, -taq 'nagen, essen' Etymologie unbekannt

Iõsta|k, gen. - $g u$ 'Netzschwimmer' Etymologie unbekannt

lõs $\mid \mathbf{u}$, gen. - $u$ 'von Mäusen oder Ratten zernagtes Getreide, Schrott' Etymologie unbekannt

lõst, gen. lõsta 'Flunder (Platichthys flesus)' laut EVS (257) eine Neubildung

lõtus, $-(s) \tilde{o}$ 'Doppelkinn' Etymologie unbekannt

lõun 'heißer Wasserdampf' Etymologie unbekannt

lõust́, gen. lõusti (vulgär) 'Plappermaul' Etymologie unbekannt

lõvi 'Löwe', wohl zu est. lovvi, gen. lõvi 'Löwe' < nd. (EES 261) (s.o.)

lõõvõrda|ma, $-q$ 'schreien, weinen' Etymologie unbekannt 
lợmbõq, gen. . lợmpõ 'Mansch' Etymologie unbekannt

lõngah(t)u|ma, -daq 'erschlaffen' Etymologie unbekannt

Iộtaŕ, gen. - $i$ 'Faulsein' Etymologie unbekannt

Iôxt|t́, gen. - $i$ 'Schlag' Etymologie unbekannt

lộ̃õksa 'geräumig' Etymologie unbekannt

mõgli|ma, - $d a q$ 'schlagen, verprügeln' Etymologie unbekannt

mõhk, gen. mõhe 'Backtrog' s.o. est. mõhk id.

mõhn, gen. mõhna 'Buckel' Etymologie unbekannt

.mõikama, mõigadaq 'beeinflussen' ? zu est. mõjuda 'beeinflussen, wirken', welches behandelt

wird

mõrdsu|k, gen. - ga 'Mörder' ? < russ. s.o. est. mõrtsukas

mõrimõrrõv 'sehr bitter' Etymologie unbekannt

mõrrõq, gen. .mõrdõ 'Sprung, Riss' ? zu est. mõra id.

mõr|rõv, gen. -õva 'bitter' ? zu est. mõru id.

.mõskma, .mõskõq 'waschen' ? zu est. mõskma id.

mõss', gen. mõssi 'Maische' Etymologie unbekannt

.mõurama, mõuradaq 'schreien, brüllen' Etymologie unbekannt

mõ o्र̃du|ma, - $d a q$ 'fördern, vorantreiben; passen' Etymologie unbekannt

nõlv, gen. nõlva 'Hang' Etymologie unbekannt

nõrg, gen. nõrõ 'Rinne' Etymologie unbekannt

nõsaraq 'Kram' Etymologie unbekannt

nõstaḱ 'Last, Bürde' Etymologie unbekannt

nốuroloogia 'Neurologie' etymologisch nicht behandelt, wohl aber neue Entlehnung

nõut.raalnõ 'neutral' etymologisch nicht behandelt, wohl aber neue Entlehnung

nọnna 'Nase, Kap, Instinkt' etymologisch unsicher, möglicher Einfluss von Kindersprache (VIITSO 1978b: 90)

nõ ỗ $\mathbf{x} \mathbf{u}$ 'gebeugt' Etymologie unbekannt

nõ ỗ tama, nõ õtaq 'Pferde rufend antreiben' Etymologie unbekannt

plõhv́, gen. plõhvi 'Schlag, Stoß, Hieb' keine Etymologie unbekannt

plõkasḱ 'Kuhglocke' Etymologie unbekannt

plõkś, gen. plôksi 'kleines Wesen' Etymologie unbekannt

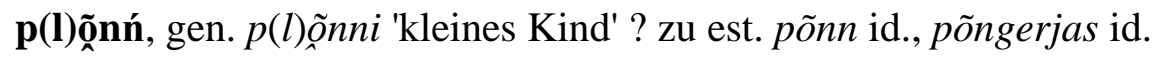

plōonsska 'Reisig' Etymologie unbekannt 
prõllaq 'jetzt' Etymologie unbekannt

.prõssama, prõssadaq 'hüpfen, hopsen' Etymologie unbekannt

(p)rônngul' 'fröhliches Gelage' Etymologie unbekannt

põgis|̃óma, -taq 'widersprechen' Etymologie unbekannt

põhn, gen. põhna 'höchster Punkt in der Wiese' Etymologie unbekannt

põka 'Traktor' Etymologie unbekannt

põko 'Schafbock' Kindersprache

põll', gen. põllõ 'Schürze' unsichere Etymologie, vgl. est. põll id. (s.o.)

põllussi(h)n 'gehemmt' Etymologie unbekannt

põllõq, .põldõ 'höchster Punkt, Plattform' Etymologie unbekannt

põpa|tama, -taq 'plaudern; rattern, knattern' Etymologie unbekannt

põr|a(ha)ma, -raq -adaq 'erschüttert werden' Etymologie unbekannt

põrba|tama, -taq 'donnern' Etymologie unbekannt

põrǵnas, gen. .põrk'na 'Karotte' Etymologie unsicher, vgl. EES (381) und SSA (II: 399)

.põrgu 'Hölle' Etymologie unsicher, vgl. est. põrgu id. sowie SSA (II: 340) und EES (400)

põtali(h)n 'in Verlegenheit, in der Klemme' Etymologie unbekannt

pymba 'gefühlloser Mensch' Etymologie unbekannt

põnndak 'kleiner Hügel' Etymologie unbekannt

rõ|ba, gen. - $b a$ 'gefallsüchtig, eitel' Etymologie unbekannt

rõhk, gen. rõha 'halbgar, ungebacken' Etymologie unbekannt

.rõhkama, gen. rõhadaq 'röcheln, rülpsen; begehren' Etymologie unbekannt

rõibõh, gen. .rõipõ 'Aas' Etymologie unsicher, vgl. est. raibe id. (EES 415)

rõika 'dünner Balken' Etymologie unbekannt

rõimõkõnõ 'Staubkörnchen, Bröckchen' Etymologie unbekannt

.rõipõlõma, rõibõldaq 'faulenzen' Etymologie unbekannt

rõp|pama, - $a d a q$ 'bemerken, erblicken' Etymologie unbekannt

.rõuhku|s 'Betonung' Laut EVS (495) eine Neubildung

rõõgi|tsõma, -tsaq -daq 'begehren, gelüsten' Etymologie unbekannt

rõõnõq, gen. ryynõ 'Flache Furche, Streifen, Linie, Welle (im Material)' Etymologie unbekannt rõnd, gen. rõnna 'Brust' Variante von estS. rind, gen. rinna id.

rỗngul', gen. $-i$ 'fröhliches Gelage, Feier' Etymologie unbekannt

.rõ̃nkõlõma, rõ ñngõldaq 'sich winden, zappeln' Etymologie unbekannt

ryyhu|ma, -daq 'leicht säuerlich werden' Etymologie unbekannt 
rọ̃̃onn, gen. rõ̃na 'halbgar, ungebacken, ungesäuert' Etymologie unbekannt

rọ̃̃óndliganõ 'gestreift, gewellt' Etymologie unbekannt

sõir, gen. sõira 'Hausgemachter Käse' < russ., s.o. est. sõir, gen. sõira 'Hausgemachter Käse mit Kümmel'

sõk|kama, -adaq 'treten; befruchten (Blumen)' Etymologie unbekannt

sysalik, 'Eidechse' Etymologie unklar, ? zu est. sisalik id.

sysarik 'Webfehler im Stoff' Etymologie unbekannt

tsõdsõ, 'Tante (Schwester des Vaters)' Etymologie unbekannt

.tsõe|tama, -taq 'Kind auf dem Feld wiegen' Etymologie unbekannt

tõha|tama, -taq 'heftig blasen, pusten, keuchen; sich kümmern, pflegen' Etymologie unbekannt

tõherus 'Ausscheidungshaufen' Etymologie unbekannt

.tõhrama, tõhradaq 'schlafen, schlummern' Etymologie unbekannt

tõhv, gen. tõhva 'Eichel' ? zu est. tõru id., etymologisch nicht bestätigt

tõia|s, gen. $-d s \tilde{o}$ 'Hohlzahn (Gewächs)' Etymologie unbekannt

tõigahta|ma, $-q$ 'kurz nicken' Etymologie unbekannt

tõir, gen. tõira 'Stange für den Heuhaufen'

tõld, gen. tõlla 'Kutsche' Etymologie unklar, ? zu est. tõld id.

tõlgu(s)s|õq, gen. -idõ 'Spreu' Etymologie unbekannt

tõlk, gen. tõlgu 'Dolmetscher' < russ., s.o. est. tõlk id.

tõlv, gen. tõlva 'Keule, Knüppel' Etymologie unbekannt

tõr|a, gen. - $a$ 'Balkon, Veranda, Empore' Etymologie unbekannt

tõrahta|ma, $-q$ 'krachend aufschlagen, erschüttert werden' Etymologie unbekannt

tõrgahta|ma, $-q$ 'zusammenzucken' Etymologie unbekannt

.tõrkuma, .tõrkudaq 'wackeln, sich schütteln, erschüttert werden' Etymologie unbekannt

tõrmihärg 'böser Mensch' Etymologie unbekannt

tõstament' 'Testament' etymologisch nicht behandelt, wohl aber neue Entlehnung

tõtul 'um die Wette' Etymologie unbekannt

.tõugjas 'Rapfen' Etymologie unsicher, s.o. est. tõugjas id.

tõõgu|tama, -taq 'nicken' Etymologie unbekannt

tõõhujuq Ausruf, um Tiere zu scheuchen, Etymologie unbekannt

tõõsi|tama, -taq 'diskutieren, streiten' Etymologie unbekannt

.tốnkama, tốngadaq 'hinken, lahmen' Etymologie unbekannt

.tôxnkõlõma, tônngõldaq 'widersprechen, streiten' Etymologie unbekannt 
võhivõõras 'wildfremd' wohl zu est. võhi- in võhivõõras id. (s.o.)

või(q) Interjektion, Etymologie unbekannt

võigista|ma, $-q$ 'diskutieren, streiten' Etymologie unbekannt

võll', gen. võlli 'Welle, Bogen' ? zu est. võll 'Welle (Maschine)', welches < nd. (s.o.)

võllas, gen. .võlla 'Galgen' ? zu est. võllas id., dessen Herkunft unbekannt ist (EES 616)

.võlśma, .võlssiq 'lügen' Etymologie unbekannt

.võotõ|(I)lõma, -llaq 'hin und her laufen, sich zu schaffen machen' Etymologie unbekannt

võra 'Gitter, Spalier' ? zu est. võrel id.

võrgu|tama, -taq 'aufmuntern, ermuntern, gesund machen' Etymologie unbekannt

võrgõq, gen. .võrkõ 'Schnur, Kordel, Leine' Etymologie unbekannt

.võrḱkma, .võrkiq 'massieren, reiben; strecken; turnen; beleben' Etymologie unbekannt

.võrksa 'lebhaft, fleißig' Etymologie unbekannt

võrokõnõ 'Bewohner/in Võrus' Etymologie unbekannt

võrŕ, gen. võrri 'Motorrad, Moped' Etymologie unbekannt

võsśa|tama, -taq '(Hunde) aufhetzen, antreiben' Etymologie unbekannt

võssõlda|ma, $-q$ 'sich herumtreiben, umherstreichen' Etymologie unbekannt

võtś Interjektion zum Scheuchen von Hunden, Etymologie unbekannt

võõgu|tama, -taq 'schaukeln, schwingen' Etymologie unbekannt

vộmmõlda|ma, - $q$ 'prügeln, hauen, schlagen' ? zu est. vemmeldama id., welches etymologisch nicht behandelt wird

vo्ñngutama 'hin und her bewegen' ? zu est. võngutama id., welches etymologisch nicht behandelt wird

.võnnkama, võngadaq 'rucken, zucken' Etymologie unbekannt

õbõrik 'Hütte, Buden, Schuppen' ? zu est. ubrik id., dessen Etymologie unklar ist (EES 574)

õeq! Interjektion, Etymologie unbekannt

õgyv', gen. õgva 'gerade, aufrecht' Etymologie unbekannt

õheq, gen. .õhkõ 'Schein, Schimmer' Etymologie unbekannt

õhi|tsõma, -tsaq -daq 'sorgen, kümmern, verwöhnen' Etymologie unbekannt

õli 'Öl', zu est. õli, welches < nd. (EES 626)

õllõ|tama, -taq 'Volkslied mit Refrain ôllõ singen' Etymologie unbekannt

.õo|tama, -taq 'Volkslied mit Refrain õo singen' Etymologie unbekannt

.õsna 'Geschwätz' Etymologie unbekannt

.ouhkõlõma, õuhõldaq 'prahlen, angeben' Etymologie unbekannt 
õuka Interjektion (Kindersprache), Etymologie unbekannt

ouru:uplanõ 'Europäer' etymologisch nicht belegt, wohl aber neuere Entlehnung

õngõrda|ma, $-q$ 'sich winden, schlängeln' Etymologie unbekannt

ợnnõ 'nur, aber, immer' Etymologie unbekannt 


\section{Anhang II: Register}

Das nachfolgende Register gibt einen Überblick über die verwendeten Belege des Korpus in alphabetischer Übersicht mit Verweis auf die entsprechende Seitenzahl.

est. hele 69

est. hiuk, gen. hiugi (dial.) 94

est. hõbe 10, 41, 50, 136

est. hobune $18,22,24,66,129$

est. hõim, gen. $-u$ 93, 191

est. hõl|p, gen. - bu 51, 111

est. hõlm, gen. - a 50,111

est. hõogata 89, 211, 217

est. hõoruda 89, 172

est. hor $\mid k$, gen. - gu 52, 112

est. hõra- 51, 111

est. horak- 51, 111

est. (h)orrn, gen. - a 52, 137

est. hüva 53

est. iga 52

est. jog $i$, gen. jõe 52, 136

est. jõhv, gen. $-e,-i$ 41, 93

est. jõlkuda 53, 138, 215

est. jõu|k, gen. -gu 94, 181, 232

est. jõuda 93, 181

est. jôlud 94, 181

est. koda, gen. koja 58, 139

estN. kodar, gen. - $a$ 58, 232

est. $k \tilde{o} h \mid t$, gen. $-u$ 49, 53, 138, 216

est. koht, gen. koha 53, 139, 220

est. kõi|k, gen. -ge 94, 212, 216

est. kõikuda 94, 191

est. kõiv, gen. - $u$ (dial. estS.) 95

est. kõla 54, 111

estN. kold, gen. kolla 54, 111

est. kõle 54, 136

est. kolm, gen. -e 54, 147

est. kõlvata 55, 111

est. kõmada 55, 137

est. kõmmeld(i) 55, 136

est. kõmpida 55, 137

est. kõn|t, gen. - di 56, 137

est. kõndida 55, 56, 137

est. kõom, gen. - $a$ 89, 211, 217

est. $k \tilde{o r} \mid b$, gen. -ve/be 56, 136

est. $k \tilde{o} r \mid s$, gen. -re 58, 136 est. kõrbeda 56, 136

estN. kord, gen. korra 56

est. kõrend, gen. - $i$ 57, 136

est. kõrge 9, 57, 136

est. korjata 57, 139

est. kõrv, gen. - a 58, 137

est. $k \tilde{o} u$, gen. -e 95, 181, 215, 230, 232

est. kõva 59, 137, 220

est. kõver, gen. - a 59, 136

est. krõmpsuda 59, 211, 216, 217

est. liuguda 96

est. lõbu 59, 111

est. lõhe 60, 136

est. lõhkeda 60, 136

est. lõhmus 60, 112

est. lõigata 95, 191

est. lõim, gen. -e 95, 198

est. lõkatada 60, 111

est. lõng, gen. - $a$ 60, 210, 216

est. lõo(ke) 49, 103, 209

est. lõog, gen. lõa 90, 172

est. lõosk, gen. - a 90, 172

est. lõots, gen. - $a$ 90, 172

est. lõppeda 61, 136, 261

est. $l \tilde{o u} \mid g$, gen. - $a$ 95, 188

est. lõu|gas, gen. -ka 96, 181

est. lõuna 96, 181

est. $m a u \mid k$, gen. - gu 97

est. mets, gen. $-a$ 64, 128

est. mõdu 61, 112

est. möga 61

est. mõis, gen. - a 96, 198

est. mõista 61, 96, 141, 212, 216

est. mõjuda 61, 138, 216, 232, 279

est. mõksuda $62,138,216$

est. mõla 16, 62, 111

est. mõlema(d) 62, 136

est. mõmiseda 62, 210, 216

est. mõni, gen. -e 62, 136

est. mõnu 63, 111

est. $m \tilde{o} \tilde{o} \mid k$, gen. -ga 90, 172 
est. $m \tilde{o} \tilde{o} \mid t$, gen. $-d u \quad 90,172,232$

est. $m \tilde{r} r \mid d$, gen. -ra 63, 111, 232

est. mõrsja 63, 137

est. mõtelda 63, 112, 215, 232, 244

est. mõtus, gen. - $e$ (dial.) 'Auerhuhn, -hahn'

64, 111

est. $n \tilde{o} \mid \lg$, gen. -le 65, 136

est. nõbu 64, 111

est. $n \tilde{o} d \mid e r$, gen. -ra 64, 111

est. nõel, gen. - a 64, 111, 232, 242

est. nõges 65, 136, 153, 224, 231

est. $n \tilde{g}$ gi, gen. -e 65, 136

est. $n \tilde{o} i \mid d$, gen. - $a$ 97, 198

est. nõmm, gen. -e 65, 210, 216, 241

est. nõr $k$, gen. -ga 66, 111

est. nõtke 66, 136, 272

est. nõu 8, 16, 41, 97, 188

est. nõuda 97, 181

est. oder, gen. odra 74, 140

est. oh|i, gen. ja 67, 139

est. ohakas 48, 103, 209

est. ôhata 67, 137

est. ôhk, gen. $\tilde{o} h u$ 67, 112

est. ôtu 68, 111

est. ohuke 68, 138, 216, 232

est. $\tilde{o} h v(a)$ 91, 172, 176

est. oige 98, 198

est. ôis, gen. ôie oile, gen. ôlme 98, 191

est. oja 69, 139

est. ok|as, gen. $-k a 67,139$

est. oks, gen. - $a$ 68, 139

est. oksendada 68, 147

est. $\tilde{o} l \mid g$, gen. $-a 69,137$

est. $\tilde{o l} \mid g$, gen. $-e$ 69, 136

est. $\tilde{o l} \mid u$, gen. -le 70, 138, 216

est. olla 70, 137, 140

est. oma 70, 139

est. ommelda 71, 136

est. ong, gen. -e 71, 136

est. onn, gen. -e 71, 136

est. $\tilde{o} \tilde{o} \mid s$, gen. -ne 71, 136, 158, 232

est. oppida 72, 137

est. $\tilde{o r} \mid s$, gen. -re 72, 137

est. ora 72, 139

est. orav, gen. - $a$ 72, 140

est. osa 73, 140

est. osi, gen. osja 74, 140

est. osta 73, 140

est. ots, gen. - $a$ 73, 140 est. otsida 12, 73, 147

est. oud(ne) 98, 181, 215, 233

est. pingutada 76

est. põd|er, gen. -ra 74, 111

est. põdeda 74, 137

est. põh|i, gen. -ja 74, 137

est. põhk, gen. $-u 75,112,130$

est. põigata 98, 198

est. põimida 99, 198, 216

est. põl|d, gen. -lu 75, 111

est. pôlata 75, 137

est. põleda 75, 210, 216

est. põlv, gen. -e 76, 137

est. põ̃on, gen. - $a$ 91, 172

est. põ̃sas 41, 76, 111, 232

est. porand 41, 77, 111

est. põrm, gen. $-u$ 77, 112, 130

est. porrn, gen. - $a$ 77, 111

est. põrsas 77, 137

est. põsk, gen. põse 77, 137

est. põtk, gen. - a 78, 137

est. põu, gen. -e 41, 78, 137, 232

est. põu|d, gen. - $a$ 99, 181

est. rohke 78, 148

est. rõht, gen. rõhu 78, 111

est. rõivas 99, 198

est. rõkkata 79, 211, 217

est. rõngas 79,111

est. rõ̃m, gen. $-u$ 92, 172

est. $r \tilde{o} \tilde{s} \mid k$, gen. - $a 91,172$

est. $r \tilde{o u} \mid k$, gen. - gu 99, 181

est. rüsa 79

est. seista 100, 194

est. sõ $\mid d a$, gen. -ja 80, 137

est. sõb|er, gen. -ra 79, 111

est. sõba 79,137

est. sõel, gen. - $a$ 80, 111, 232

est. sõge 80,137

est. sõim, gen. -e 100, 198

est. sõita 100, 198

est. $s \tilde{o l} \mid g$, gen. -le 80, 137

est. sõlm, gen. -e 81, 137, 151

est. sõmer, gen. - $a$ 81, 137

est. sõna 81, 210, 216, 241

est. sõnn, gen. - $i$ 81, 137

est. sõnnik, gen. $-u$ 50, 82, 137

est. sõ̃m, gen. $-u$ 92, 172

est. sõor|e, gen. -me 92, 172, 231

est. sõ̃rd, gen. - $u$ 103, 209 
est. $s \tilde{r} r \mid g$, gen. - $a$ 82, 137, 220

est. sõre 82, 137

est. sõrm, gen. -e 82, 137

est. sõsar 27, 83, 111

est. sõstar 83, 111

est. sõtkas 83, 138

est. sõtkuda 83, 138, 216, 232

est. sõuda 22, 100, 181

est. suuta 100

est. süva 86

est. teine 101, 199

est. tõbi, gen. tõve 83, 137

est. tõbras 84,111

est. tohtida 84, 148, 153, 232

est. tõke, gen. tõkke 84, 137

est. tõmmata 84, 111, 134

est. tõnguda 84, 138, 141, 215

est. tõotada 104, 209

est. $t \tilde{r} r \mid s$, -re 85, 165

est. tõri, gen. tõrve 85, 137

est. tõrjuda 85, 138, 216

est. tõru 11, 14, 85, 111, 281

est. tõrv, gen. - $a$ 85, 111

est. tõsi, gen. tõe 86, 137

est. $t \tilde{o u} \mid k$, gen. -gu 101, 181

est. tõug, gen. tõu 101, 181

est. tõugata 101, 181, 185

est. tõusta 103

est. tülts, gen. - $a 85$

est. tüvi, gen. $-e 86$

est. võhk, gen. võha 86,111

est. voi 102, 201

est. või 102, 198, 212, 216

est. võida 102, 198

est. võl $\mid g$, gen. $-a$ 87, 111

est. vôlu 87, 111, 239

est. võnnu- 87,111

est. võ̃ras 93, 172

est. võr|d, gen. -ra 87, 111

est. $v \tilde{o r} \mid k$, gen. - $g u$ 87, 111, 232

est. võrkida 88, 111

est. võrsuda 88, 111

est. võsa 88, 111, 123, 265

est. võtta 88,138

estS. ? egä, ogga 52

estS. hää $~ h \ddot{u}(v) \ddot{a} 53$

estS. häilmü, häiermü 98, 196

estS. helle 69

estS. (h)õhk, gen. (h)õhu 67, 133
estS. (h)ôhv, gen. (h)õhva 91, 176, 177

estS. hõim, gen. hõimo 93, 196

estS. hõlm, gen. hõlma 50,133

estS. hõlp, gen. hõlbu 51, 133

estS. hõ õgama, II. Infinitiv hõoadaq;

hõõhkama, II. Infinitiv hõohadaq 89,

211,217

estS. hõor $(d) m a$, II. Infinitiv hõ õ $r(d) u q$ 89, 177

estS. hо̃ро 49, 50, 163, 235

estS. ho|põn', gen. -bõsõ hobõ|nõ, gen. sõ 66,129

estS. horra(s)silm 51, 133

estS. horak 51, 133

estS. jõgi, gen. jõõ 52, 163

estS. jôhv', gen. jốhvi; jõuh, gen. jõuhõ 93, 186, 235

estS. .jõlkma, II. Infinitiv jõlkuq 53, 164, 215

estS. .joudma, II. Infinitiv (.)joudag; .jõudma, II. Infinitiv (.)jõudaq 93, 185

estS. jõuk, gen. jõugu 94, 186

estS. joul, gen. joulu; jõul, gen. jôlu 94

estS. koda, gen. kua 58

estS. $k \tilde{\alpha} \mid i k^{\prime}$, gen. - $g \tilde{o}$ 94, 212, 216

estS. .kõikma, II. Infinitiv .kõikuq 94, 196, 236

estS. kõiv, gen. - $u$ 198, 207

estS. kõla 54, 133

estS. .kõlbama 55, 133

estS. kõlla|nõ, gen. -dsõ 54, 111, 133

estS. kõllõ 54, 163

estS. kolm, gen. kolmõ 54, 169

estS. .kõmpma, II. Infinitiv .kõmpiq;

.kõmpsíma, II. Infinitiv .kõmpsiq 55, 164

estS. .kõnd'ma, II. Infinitiv .kõndiq 55, 164

estS. koomõq, gen. kuumo; kohmõq, gen.

.kohmõ 89, 211, 217

estS. .kõrbõma, II. Infinitiv kõrbõdaq kõrvõdaq 56, 163

estS. kõrd, gen. kõrra 56, 111, 133

estS. kõreńd 57, 163

estS. .korgõ 57, 163, 169

estS. .korjama, II. Infinitiv koradaq 57

estS. kõrs', gen. kõrrõ 58

estS. kõrv, gen. kõrva 58, 164

estS. $k \tilde{o t} \mid t$, gen. $-u$ 49, 53, 164, 216

estS. kõ|tar', gen. -dara 58, 111, 133, 232

estS. kõva 59, 163, 220 
estS. kõvve 49, 95, 186, 215

estS. $k \tilde{v} v \mid v \tilde{o r}{ }^{\prime}$, gen. - $\tilde{\text { ora }} 59,163$

estS. kuht, gen. koha kuha 53, 165, 235

estS. lang, gen. langa 60

estS. lõbu 59, 133

estS. lõhekala; lõh|i, gen. -e 60, 163

estS. lôhkõ- 60, 163

estS. lõhmus 60, 133

estS. lốig, gen. lõia 90, 177, 235

estS. .lõikama, II. Infinitiv lõigadaq 95, 196

estS. lõim', gen. lõimõ 95, 207

estS. lõiv, gen. lõivo; lõokõ|nõ, gen. -sõ;

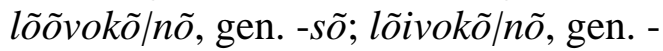
sõ 103

estS. lõõts, gen. lõodsa 49, 90, 177

estS. . lôpma, II. Infinitiv lõppõq 61, 163, 235

estS. lõug, gen. lõvva 95, 185, 190, 235

estS. lõugõq, gen. .lõukõ 96, 186

estS. lounõq, gen. .lôuñ ; lounaq, gen.

.lõuna; lõuna|k, gen. -gu 96, 186

estS. $m \tilde{o} d u$ 61, 133

estS. .mõisa 96, 206

estS. .mõistma, II. Infinitiv .mõistaq 96, 212, 216

estS. mõla 62, 133

estS. mõlomb $\mid a q \quad 62,163$

estS. mõmis|ôma, II. Infinitiv -taq 62, 210, 216

estS. mõni, gen. mõnõ 62

estS. mõno 63, 133

estS. mõ õk, gen. mõoga 90, 177

estS. mõo|ma, II. Infinitiv -daq 49, 61, 164, 216

estS. mõot, gen. mõ odu 90, 177

estS. môrd, gen. mõrra 63, 133, 235

estS. .mõrsja 63, 164

estS. .mõtlõma, II. Infinitiv mõtõldaq 63, 134, 215, 235, 244

estS. mõts, gen. mõtsa 64, 129, 134, 217

estS. mõtus 48, 64, 133

estS. nõder, gen. nõdra 64, 133

estS. nõgi, gen. nõ $\tilde{o}$ 65, 163

estS. nõgõl; nõkl, gen. nõgla 64

estS. nõid, gen. nõia 97, 207

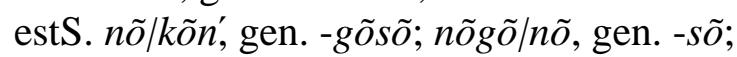

$n \tilde{o} \mid k \tilde{o} s^{\prime}$, gen. - $-g \tilde{o}(s) s \tilde{o} \quad 65,164$

estS. nõlo 65, 163
estS. nõmm gen. -e 65, 210, 216, 241

estS. nõrk, gen. nõrga 66, 133

estS. nõs|óma, II. Infinitiv -taq 103

estS. .nõtskõ 66, 163

estS. nõudma, II. Infinitiv (.)nõudaq 97, 186

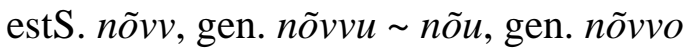
97, 190

estS. oddak otak 68, 113, 133

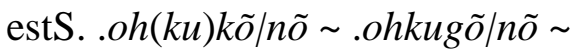

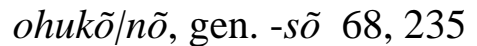

estS. oha|k, gen. - gu 67

estS. .õhkama, II. Infinitiv õhadaq 67, 164

estS. .oigo 98, 207

estS. oja; uja 69

estS. olg, gen. ola 69

estS. olg', gen. olõ 69, 163, 169

estS. olõma, II. Infinitiv ollaq 70

estS. oluq, gen. ollõ 70, 169

estS. õng', gen. õngõ 71, 163

estS. onnn', gen. ônno 71, 163, 235

estS. .op'ma, II. Infinitiv oppiq 72, 163, 169

estS. ora 72

estS. orn, gen. orrna 52

estS. or rav, gen. -ava 72

estS. ors', gen. orrõ 72, 169

estS. osa 73

estS. osi, gen. os'a 74

estS. ossa, gen. ossa 68

estS. ossõnda|ma, II. Infinitiv - $q$ 68, 169

estS. .ostma, II. Infinitiv .ostaq 73

estS. ots, gen. otsa 73

estS. .ots'ma, II. Infinitiv .otsiq 73, 169

estS. pal|ama, II. Infinitiv -laq 75

estS. pingu|tama, II. Infinitiv -taq 76

estS. põ|dorma, II. Infinitiv -tõ 74,163

estS. põdor $\sim$ põtr, gen. põdra 74, 133

estS. põhi, gen. põh'a 74, 164, 236

estS. põhk, gen. põhu 75, 133

estS. põld, gen. põllu 75, 133

estS. .põlgma, II. Infinitiv .põlgõq 75, 163

estS. põlv', gen. põlvõ 76, 163

estS. põõn, gen. põ̃na 91, 177

estS. põrm, gen. $-u$ 77, 134

estS. põrma(n)d 77, 133

estS. põrn, gen. põrna 77, 133

estS. põrss, gen. .põrsa 77, 164

estS. põsk, gen. põsõ 77, 163 
estS. põud, gen. põvva 99, 186

estS. .rohkõ 78, 169

estS. rõivas, gen. .rõiva 99, 207

estS. rõkkama 79, 211, 217

estS. rộngas, gen. .rônnga 79, 133

estS. rõõm, gen. rõ̃mu 92, 177

estS. rõ õosk, gen. rõõsa 91, 177

estS. rõuk, gen. rõugu 99, 186

estS. .saisma, II. Infinitiv (.)saistaq 100, 196

estS. sõba 79, 164

estS. sõbõr sõbr, gen. sõbra 79, 133

estS. sõda, gen. sõa 80, 164

estS. sõgõl; sõkl, gen. sõgla 80, 133

estS. .sõitma, II. Infinitiv .sõitaq 100, 207

estS. .sõkma, II. Infinitiv sõkkuq 83, 164, 216

estS. sõkõ 80, 163

estS. sõlg, gen. sõlõ 80, 163

estS. sõlm', gen. sõlmõ 81, 163

estS. sõm|mõr', gen. -õra 81, 163

estS. sõna 81, 210, 216, 241

estS. sõnn, gen. $-i \quad 81,164$

estS. sõnnik 50, 82, 164

estS. sõ̃m, gen. sõ̃mu 92, 177

estS. sõ̃rd 103

estS. sõ̃rmas, gen. sõ õrma; sõ̃rmõs, gen.

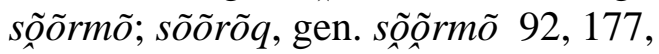
235

estS. sõrg, gen. sõra 82, 164, 220

estS. sõrm', gen. sõrmõ 82, 164

estS. sorrõ 82, 169

estS. sõ sar' 83, 133

estS. sõtkas, gen. .sõtka 83, 164

estS. .sõudma, II. Infinitiv (.)sõudaq 100, 186

estS. .sutma, II. Infinitiv suttaq 100

estS. süvä 86

estS. $t \tilde{o} \mid b i$, gen. -võ/bõ 83, 164

estS. tõbras, gen. .tõpra 84, 133

estS. .toht'ma, II. Infinitiv .tohtiq 84, 170

estS. .tõmbama 84, 133

estS. .too|tama, II. Infinitiv -taq; toovotama, II. Infinitiv -taq; .tõo|tama,

II. Infinitiv -taq 104

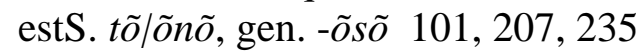

estS. .torrdo 85, 164

estS. tõrjuda 85, 164, 216

estS. tõrv, gen. tõrva 85,133
estS. tõtõ 86, 164

estS. tõug, gen. tõvvu 101, 186

estS. .toukama, II. Infinitiv tougadaq; .tuukama, II. Infinitiv tuugadaq 101, 187

estS. tüvi, gen. -e 86

estS. uhak; (.)ohtjas; (.)uhtjas; (.)ohk'mas;

(.)uhk'mas; (.)oht'mas; (.)uht'mas; uhas 103

estS. uhi, gen. uha $\sim$ ohi, gen. oha 67

estS. uma 70, 165

estS. .umblõma 71, 166, 224, 235

estS. vai 102, 212

estS. võhaleht 86,133

estS. võhl, gen. võhlu 49, 87, 133

estS. võid, gen. võiu 102, 207

estS. .võima, II. Infinitiv .võidaq 102, 207

estS. võlg, gen. võla 87, 133

estS. võoras, gen. võôra 93, 177

estS. võrk, gen. võrgu 87, 133, 236

estS. .võrsu|ma, II. Infinitiv -daq 88, 133

estS. võso 50, 88, 133, 236

estS. .võtma 88, 164

liv. aim 93, 196

liv. $\bar{e} d r o \tilde{m} 98$

liv. é 'ga 67,127

liv. ? erā 51, 127

liv. ería-māra 51, 127

liv. jeka 52

liv. joudõ 93, 185

liv. jo'ug 52

liv. jõ'ugi 93, 184

liv. jõugõ 94, 211

liv. jõvā 53, 210

liv. ? keldarikki 54, 132

liv. kõ'ddõrz 58, 127

liv. $k \tilde{o}^{\prime} l \quad 54,157$

liv. kõlā 54, 127

liv. kõlbõ 55, 127

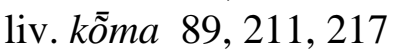

liv. kỗrda 56, 127

liv. kõ'urõ 59, 157

liv. kõuvõ 95, 205, 234

liv. kõva 59, 157

liv. krệmpš 59, 211, 216, 217

liv. kuodā 58

liv. ku'odi 53, 158

liv. kuolm 54, 158

liv. kūora 58 
liv. kuorbõ 56, 158

liv. kuordõ 57, 158, 234

liv. kuor'́n 57,158

liv. lānga 26, 60

liv. lỗga 95, 190

liv. lōkandõks 96, 185, 217

liv. lỗnag 96, 184

liv. loptõ 61, 162

liv. lỗtstõ 90, 176

liv. lõuglõ 96, 211

liv. lūoima 95, 234

liv. $m \tilde{g} g \bar{a} \quad 61,210,216$

liv. moistz/muoista 96

liv. möizõ/muoiza 96

liv. moijjõ 61,159

liv. $m \overline{\tilde{o}} k 90,176$

liv. $m o \tilde{k} k a$ 97, 211, 216

liv. mólmõd 62,159

liv. mỗrda 63,127

liv. mõtlõ $63,128,215,234,244$

liv. mõtsā $64,128,134,217,234$

liv. mõtūks 46, 64, 127

liv. münda 62,160

liv. nõ'ddõr 64, 127

liv. nõ'ggõl 64, 127

liv. nolgõ 65,162

liv. nỗrka 66, 127

liv. nõtkõ 66, 157

liv. no'ugõd 65

liv. $n \tilde{o}^{\prime} v 97,190$

liv. num 65

liv. $n \bar{u} z \tilde{o} 103$

liv. ón'bbi 66, 127, 129, 234

liv. ó'bdõ 50, 157, 234

liv. ỗ'dõg 68, 113, 127

liv. ? oga-pä 67

liv. $\overline{\tilde{o} g \tilde{o}} 89,211,217$

liv. õigi 98,205

liv. oksā 68

liv. oksnõ 68, 162

liv. ó'ldzo $69,128,216$

liv. ôlma 50, 127

liv. ỗn 71, 157, 234

liv. орро̃ 26, 72, 162

liv. $\tilde{\tilde{o}} r \tilde{o} 89,176$

liv. ó 'uv 91, 176

liv. pa'llõ 75

liv. põda 99, 184

liv. pó'ddõ 74, 159 liv. põ'ddorrz 9, 74, 127, 234

liv. pöimõ 99

liv. $p \dot{o}^{\prime} j 78,159$

liv. $p \overline{\tilde{o}}^{\prime} k t \quad 75,127,234$

liv. pólgõ 75, 159

liv. põ̃n 91, 176

liv. põnktõ 76, 210, 216

liv. pỗrand 77, 127, 273

liv. põrm 77, 127

liv. pỗrna 77, 127

liv. posk 77, 159

liv. pótkāstõ 78, 159

liv. põ̃zõ 76, 127, 234

liv. pü'oj 74, 158

liv. pūola 76, 158

liv. pūoraz 77, 158

liv. rõkkõ 79, 211, 217

liv. rỗm 92, 176

liv. rỗskõ 91, 176

liv. rõ'ut 78, 79, 127

liv. rõza 79,210

liv. seitz 100, 204

liv. sēmda 92, 177, 217

liv. sõbrā 79, 127

liv. so'gda 80,162

liv. sõ'ggõl 80,127

liv. sõitõ 100, 212, 216, 217

liv. sõna $81,210,216$

liv. sỗrga 82,157

liv. sỗrmõz 92, 176, 234

liv. sõudõ 100, 184

liv. sõzār 83,127

liv. sumār 81,159

liv. suodā 80, 158

liv. suolg 80, 158

liv. suolm 81, 158

liv. suorm 82, 159

liv. suotkõ 83, 159

liv. $t e^{\prime} b, t \ddot{\prime}^{\prime} b 83$

liv. tevk, teuk 101, 184

liv. to'ggõd, to'ugad 84

liv. tôitõ 104

liv. tỗlza 85, 210, 216

liv. tõmbõ 84, 127

liv. tỗra 85,127

liv. $t \bar{o} r d a z \quad 85,157$

liv. tốrmõz 14, 85, 127

liv. tõurõz 102, 211

liv. $t \tilde{o}^{\prime} v 86,210$ 
liv. $t \tilde{o} v \bar{a} \quad 86,210$

liv. $t \bar{u}$ 'odo 84,159

liv. tuoi 101

liv. $t^{u} o^{\prime} i z ̌ \quad 86,159$

liv. $u^{\prime} m$ 70, 159

liv. umblõ 71, 159, 224, 234

liv. ? ū'ogõz/ūogaz/u'ogaz 103

liv. $\bar{u}^{\prime}$ olmõz 67,158

liv. v(u)ojā, vojja 69, 159

liv. ve îh 102, 205

liv. vêrka 88, 114, 127

liv. vo'ddõrz/v(u)o'draz 74, 159

liv. või 102, 212, 216

liv. võ̃idag 102, 205

liv. võidõ 102, 204

liv. $v \dot{o}^{\prime} l 70,159$

liv. võ'l $87,127,239$

liv. vó̀lda 70, 159

liv. vólg 69, 159

liv. vỗlga 87, 127

liv. von 71, 234

liv. vöntsa/vūontsa 73, 159

liv. vorra 72,159

liv. vơrābõz/v(u)orāboz, 72, 159

liv. võrgõ 87, 127

liv. vörna 52, 159

liv. vỗrõz 93, 176

liv. vorrž 72, 159

liv. vostõ 73,159

liv. votšo 73,159

liv. võttõ 88, 157, 159, 216

liv. voz $\bar{a} 73,159$

liv. voža 74,159

liv. võza $88,123,127$

wot. ed'jelmo/ed'd'ôlmo 98

wot. eliä 69

wot. erkku 52, 125

wot. helppo 14, 51, 125

wot. jõka 52, 150

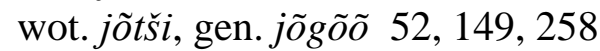

wot. jõukku 94, 183

wot. jõulu 94, 183

wot. jõutaa 93, 183

wot. jõv|i, gen. -ii 93

wot. keikab 94, 195

wot. kõhta 53, 150, 220

wot. kõhu|z, gen. -hsõõ 53, 150, 216

wot. kõikki, gen. kõikõo 94, 212, 216

wot. koivu 95, 204 wot. kõla 54, 149, 150

wot. kõlmõD 54, 149

wot. kõlta 54, 122

wot. kõlvata 55, 122

wot. kõntaassa 55, 56, 150

wot. kõrjata 57, 150

wot. kõrkõa 43, 57, 149, 151

wot. kõrõta 57, 149

wot. kõrsi, gen. kõrrõ õ 58, 149

wot. kõrta 56, 122

wot. kõrva 58, 150

wot. kõta 58, 150

wot. kõtara 58, 122

wot. kõva 59, 150, 220

wot. kovera 59, 156

wot. lekko 60, 125

wot. liukua 96

wot. lõhgõta 60, 149

wot. lõhi, gen. lõhõo 60, 149

wot. lõikata 95, 194

wot. loim|i, gen. -o 95, 202

wot. lõyka 60, 210, 216

wot. lõoka 90, 174

wot. lõoska 90, 174

wot. lõõtsua 90, 174

wot. lõpõttaa 61,149

wot. lõuka 95, 188

wot. lõunaD 96, 183

wot. meno 63, 126

wot. mettsä 64,128

wot. mõissaa 96, 212, 216

wot. mõiza 96, 202

wot. mökisä 61

wot. môla 62, 122

wot. mõlõpaD 62,149

wot. mõni, gen. mõnõ 62,149

wot. mõókka 90, 174

wot. mõ̃tto 90, 174

wot. mõrta 63,122

wot. mõtõlla 63, 122, 215, 233, 244

wot. mõtu 61, 122

wot. nigla 42, 64, 123, 233, 242

wot. nõdra 64, 122

wot. nõisa 103, 209

wot. nõita 97, 202

wot. nõkõn 65,150

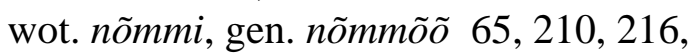
241

wot. nõrkka 66, 122 
wot. nõtši, gen. nõgõ $\quad 65,149$

wot. nõvvo 97, 188, 233

wot. $\tilde{o g a z} 67,150$

wot. ôhja 67,150

wot. ôhsa 68,150

wot. ôhsõta 68,149

wot. ôhtago $68,113,122$

wot. ôhuD 68, 150, 216

wot. ôhva 91, 174, 176

wot. ôikõa 98, 202

wot. oimo 93, 194

wot. oja 69,150

wot. ôlla 70, 150

wot. ôlma 50, 122

wot. ôltši, gen. ôlgõo 69,149

wot. $\tilde{o} l u \mid D$, gen. $-u 70,216$

wot. oma 70,150

wot. ómmõlla 71, 149

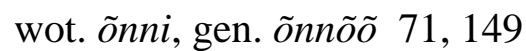

wot. ontši, gen. ondžõ $\tilde{o} 71,149$

wot. $\tilde{o} \tilde{o} h k o$ 103, 209

wot. õortaa 89, 174

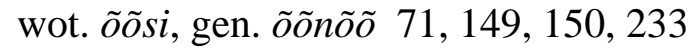

wot. оро̃a 42, 50, 149, 151, 233

wot. opõñ 66, 129

wot. oppõa 26, 72, 149

wot. ora 72, 150

wot. orahtaa 51, 122

wot. Orava 72,150

wot. orrsi, gen. ôrrõ̃ 72,149

wot. osa 73, 150

wot. ossaa 73, 150

wot. ottsa 73, 150

wot. ottsia 73, 150

wot. ozra 74, 150

wot. ozża 74,150

wot. piina 91, 175, 233

wot. põdra 74, 122

wot. põhja 74, 150

wot. põhku 75, 122, 130

wot. põimia 99, 202, 216

wot. põitši 98, 202

wot. põlgõt/taa 75, 149

wot. põlõa 75, 210, 216

wot. põlto 75, 122, 153

wot. põlvi, gen. põlvõ̃ 76, 149

wot. põ̃zaz 76, 122, 233

wot. põrmata 77, 122

wot. põrmu 77, 122, 130 wot. põrna 77, 122

wot. põrzaz 77, 150

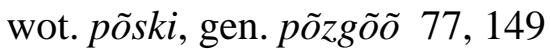

wot. põtkõa 78, 149

wot. põuta 99, 183

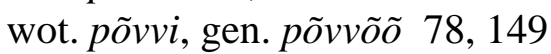

wot. rõhgaa 78, 150

wot. roivaz 99, 204

wot. rõygaz 79, 122

wot. rõõska 91, 174

wot. rõukko 99, 183

wot. rüsä 79

wot. sẹr (a)med 92, 174, 233

wot. (sev)võrta 87, 122

wot. sigla 80,123

wot. sõbra 79, 122

wot. sõim|i, gen. -õ̃ 100, 202

wot. sõisa 100, 194

wot. sõittaa 100, 202

wot. sõkõa 80, 149

wot. sõlm|u, gen. - $и u$ 81, 149, 233

wot. sõmõrõ 81, 149

wot. sõna 81, 210, 216, 241

wot. sõpa 79, 150

wot. sorkka 82, 152, 220

wot. sõrmi, gen. sõrmõ̃ 82, 149

wot. sorõa 82, 156

wot. sõrto 103

wot. sõssar 83, 114, 122

wot. sõta, gen. sõaa 36, 80, 150

wot. sõtkoa/-ua 83, 150, 216, 233

wot. sõutaa 100, 183

wot. sõzar 83, 122

wot. süvä 86

wot. tõbraz 84, 122

wot. tõgo, gen. tõkõo 84, 149

wot. tõhtia 84, 150, 216, 233

wot. tõin, gen. tõizõ̃ 101, 202

wot. tõivoa 104

wot. tõmmata 84, 122

wot. tõygata 84, 141, 150, 151, 215

wot. tõrva 85, 122

wot. tõsi, gen. tõ̃ 86, 149

wot. tõukata 101, 183, 185

wot. tõukka 101, 182, 183

wot. tõuko 101, 183

wot. turu 14, 85, 123

wot. tüvi, gen. tüvee 86

wot. $\ddot{u} v \ddot{a} 53$ 
wot. veija/võitaa 102, 202

wot. ventõizõ̃ 87,126

wot. võhka 86, 122

wot. või 102, 202, 212, 216

wot. võlka 87, 122 wot. võ̃raz 93, 174

wot. võrkko 87, 122

wot. võso 88, 122, 233

wot. võttaa 88,150 